\title{
14. CENOZOIC CALCAREOUS NANNOFOSSILS FROM DEEP SEA DRILLING PROJECT LEG 75, SOUTHEAST ATLANTIC OCEAN ${ }^{1}$
}

\author{
John C. Steinmetz, ${ }^{2}$ Department of Marine Science, University of South Florida, St. Petersburg, Florida \\ and \\ Herbert Stradner, Geologische Bundesanstalt, A-1031 Vienna, Austria
}

\begin{abstract}
Sediments from the three DSDP Leg 75 sites in the southeast Atlantic Ocean collectively contain a calcareous nannofossil record of almost the entire Cenozoic. Two rotary-cored holes and six hydraulic piston-cored holes were drilled. The youngest sediment recovered is Recent from the Emiliania huxleyi Zone, and the oldest sediment is at the Cretaceous/Tertiary boundary in Hole $530 \mathrm{~A}$. The nannofossils are generally common to abundant and moderately well-preserved. One new species is described, Biantholithus astralis nov. spec.
\end{abstract}

\section{INTRODUCTION}

Cenozoic sediments were recovered on Deep Sea Drilling Project (DSDP) Leg 75 at all three sites occupied in the Angola Basin and on the Walvis Ridge in the southeast Atlantic Ocean (Fig. 1). Only two of the eight holes were rotary-cored; the remainder were hydraulic piston cored (HPC). The oldest Cenozoic sediments recovered are basal Paleocene in age in Hole 530A. Cenozoic sediments generally contain common to abundant, moderately well-preserved nannofossils. Species assemblages are relatively diverse, permitting use of low-latitude zonations for biostratigraphy. Reworked older taxa were rarely encountered.

\section{METHODS}

Smear slides of samples were prepared employing commonly accepted techniques suggested by Bramlette and Sullivan (1961) and Hay (1961, 1965). The slides were examined under both cross-polarized and phase-contrast light at $\times 1200$ magnification. The scanning electron microscope was used to confirm the presence of Emiliania huxleyi in cores where it was suspected. The Cenozoic nannofossils considered in this chapter are listed in the Appendix along with an index to the plates. The presence and abundance of all nannofossils observed were recorded in range charts (see Tables 1 through 4). Abundance estimates of the total number of taxa present and of individual taxa in the assemblage were made according to the method introduced by Hay on Leg 4 (1970). The abundance is estimated by the order of magnitude of its frequency per field of view at $\times 1200$. This is expressed in terms of an exponent to the base ten. Table 5 lists the frequency estimate designations used and their meanings. The overall preservation of nannofossil assemblages was recorded using one of three letter designations instituted on Leg 49 (Steinmetz, 1979).

$\mathrm{G}=\mathrm{Good}$ preservation. Fossils lack evidence of dissolution or overgrowth.

$\mathrm{M}=$ Moderately good preservation. A majority of the specimens are slightly etched. Fine structures are missing, but no diagnostic changes of form are evident in light microscopy. Rim margins of placoliths are slightly serrate. All taxa may be easily identified. Diversity is normal.

$\mathrm{P}=$ Poor preservation. A majority of the specimens are deeply etched. Identity of many centerless and fragmented specimens is questionable. Diversity is low.

\footnotetext{
${ }^{1}$ Hay, W. W., Sibuet, J.-C., et al., Init. Repts. DSDP, 75: Washington (U.S. Govt. Printing Office).

2 Present address: Denver Research Center, Marathon Oil Company, Littleton, Colo-
}

\section{NANNOPLANKTON ZONATION}

Three nannoplankton zonations were employed to make zonal and geological age assignments of the samples. The Standard Nannoplankton Zonation of Martini (1971) was used for the shipboard and site reports. After closer inspection of the samples in shore-based studies, we found that Bukry's (1973, Leg 15; 1975, Leg 32) and Ellis' (1979; 1982, Leg 60) zonations offered higher resolution. Okada and Bukry (1981) introduced code numbers to the low-latitude zonation of Bukry $(1973,1975)$. Table 6 shows a correlation of the calcareous nannoplankton zonation schemes proposed by Martini (1971), Bukry $(1973,1975)$, and Ellis $(1979,1982)$, as well as their respective code numbers which are used throughout this report. Table 6 also shows the geologic age and nannofossil zone assignments of Leg 75 cores correlated with the zonations.

\section{Site $\mathbf{5 3 0}$}

Site $530\left(19^{\circ} 11.26^{\prime} \mathrm{S}, 9^{\circ} 23.15^{\prime} \mathrm{E}\right.$; water depth, 4629 $\mathrm{m})$ is located in the southeastern corner of the Angola Basin, about $20 \mathrm{~km}$ north of the Walvis escarpment, near the eastern end of the Walvis Ridge. Three holes were drilled at Site 530. Hole 530 was drilled using a standard rotary coring assembly, but was terminated (115 $\mathrm{m}$ below the seafloor) by technical failure. Hole 530A (1121 m penetration) was drilled using a standard rotary coring assembly. Hole $530 \mathrm{~B}$ (180 m penetration) was cored using the hydraulic piston coring (HPC) device first employed on Leg 65.

Four sedimentary units were described in the Cenozoic sediments at Site 530. Unit 1 consists of Holocene to early Pleistocene diatom nannofossil ooze, marl, and debris-flow deposits to a depth of $110.0 \mathrm{~m}$ sub-bottom. Unit 2 consists of early Pleistocene to late Miocene nannofossil clay, marl, ooze, and debris-flow deposits from 110.0 to $277.0 \mathrm{~m}$ sub-bottom. Unit 3 consists of late Miocene to Oligocene red and green muds from 277.0 to $467.0 \mathrm{~m}$ sub-bottom. Unit 4 consists of Eocene to Danian multicolored mudstone, marlstone, chalk, and clastic limestones from 467.0 to $592.6 \mathrm{~m}$ sub-bottom. 


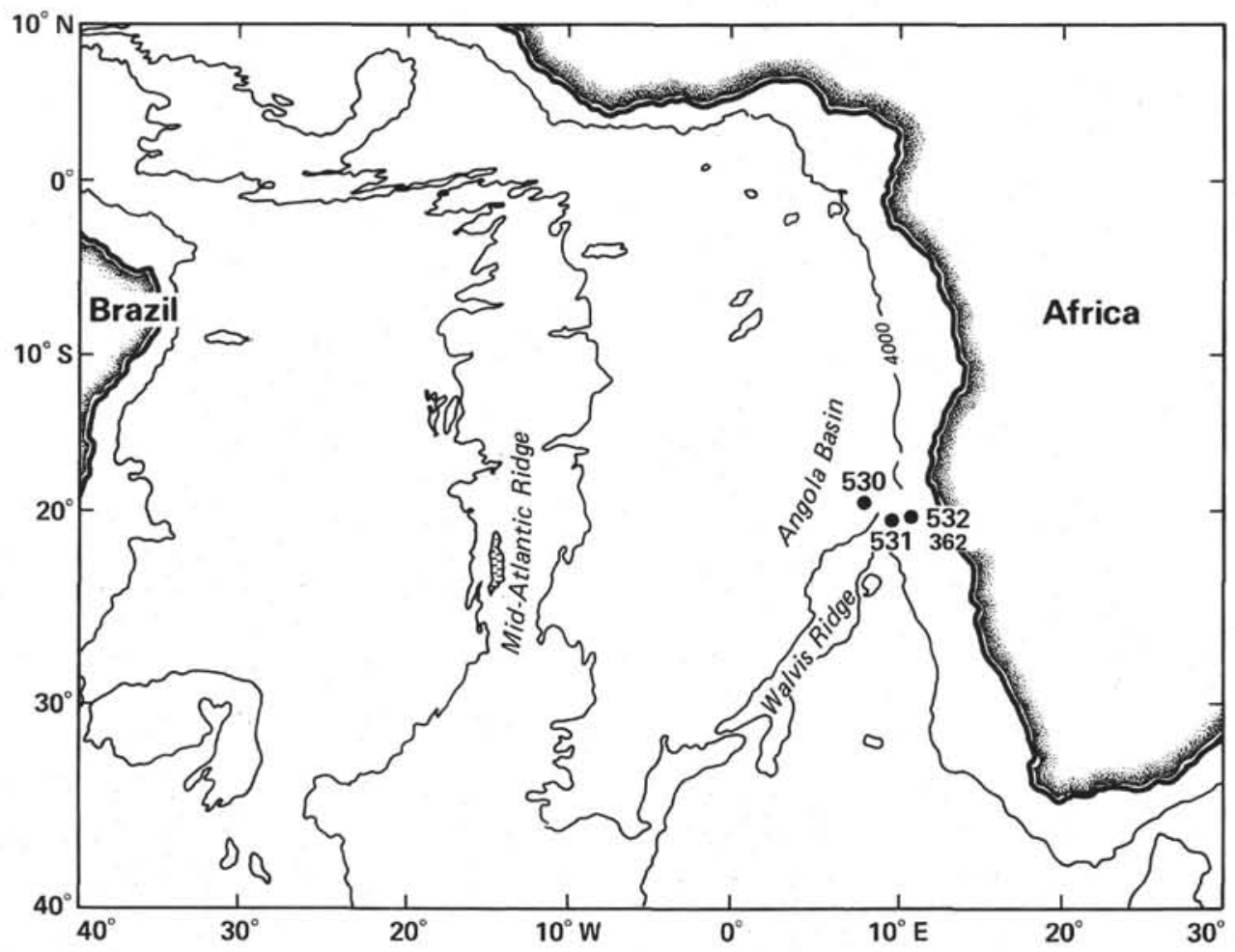

Figure 1. Location of Sites 530-532, Leg 75.

In Holocene through late Miocene sediments, nannofossils are generally common to abundant in number and show moderate to good preservation. The early and middle Miocene is largely unzoned because of repeated barren sections. The remainder of the Tertiary record is well-zoned using common to abundant numbers of moderately to poorly preserved nannofossils.

Hole 530. Only two cores were recovered from Hole 530. The presence of Emiliania huxleyi in Core $1(0.0 \mathrm{~m}$ to $1.5 \mathrm{~m}$ sub-bottom) indicates the Holocene $E$. huxleyi Zone (NN21, CN15, WPN32). Core 2 (taken after washing to $115.5 \mathrm{~m}$ sub-bottom) contains an assemblage characteristic of the early Pliocene Discoaster asymmetricus Subzone (upper NN15, CN11b, WPN28b): Calcidiscus macintyrei, D. brouweri, D. pentaradiatus, D. surculus, D. tamalis, and Sphenolithus abies. Gephyrocapsa caribbeanica is present as a Pleistocene contaminant.

Hole 530A. Coring in Hole 530A was begun after washing to $125.0 \mathrm{~m}$ sub-bottom. Core 1 to Core 4 , Section $4(125.0 \mathrm{~m}$ to $158.7 \mathrm{~m})$ are latest early Pliocene age, Reticulofenestra pseudoumbilica Zone (NN15, CN11, WPN28). Discoaster asymmetricus Subzone (upper NN15, CN11b, WPN28b) is delimited on the top by the last occurrences of Sphenolithus abies and $R$. pseudoumbilica, and on the bottom by the first occurrence of $D$. tamalis, not by the acme of $D$. asymmetricus as proposed by Bukry (1973). The $S$. neoabies Subzone (lower NN15, CN11a, WPN28a) occurs from 4-4 to 5,CC $(158.7 \mathrm{~m}$ to $172.5 \mathrm{~m})$ and is delimited on the top and bottom, respectively, by the first occurrence of $D$. tamalis and the last occurrence of Amaurolithus tricorniculatus.
Only one distinct subzone is discernable in the $A$. tricorniculatus Zone (NN12-14, CN10, WPN27). The Ceratolithus rugosus Subzone (NN13-14, CN10c, WPN27c) occurs between the last occurrence of $A$. tricorniculatus at 5,CC $(172.5 \mathrm{~m})$ and the first occurrence of $C$. rugosus at 7-4 $(187.2 \mathrm{~m})$. Below this is a section with mixed subzone indicators (NN12, CN10a-10b, WPN27a-27b) which extends down to the last occurrence of $D$. quinqueramus at $8, \mathrm{CC}(201.0 \mathrm{~m})$.

The late Miocene D. quinqueramus Zone (NN11, CN9, WPN26) occurs from $8, C C$ to $15, \mathrm{CC}(201.0 \mathrm{~m}$ to $267.5 \mathrm{~m}$ ) and is divisable into two subzones: the $\mathrm{A}$. primus Subzone (upper NN11, CN9b, WPN26b) and the $D$. berggrenii Subzone (lower NN11, CN9a, WPN26a). The first occurrence of $A$. primus (13,CC, $248.5 \mathrm{~m}$ ) separates the two subzones. From 15,CC to $18-4$ (267.5 m to $291.7 \mathrm{~m}$ ) the middle to late Miocene interval is compressed, and individual zones or subzones are not resolvable. Indicators of Subzones CN5b to CN8b (NN7 to NN10, WPN22b to WPN25b) are absent. Much of the middle Miocene is barren and unzoned from Sections $18-5$ to $21-5$ (293.2 $\mathrm{m}$ to $321.7 \mathrm{~m})$. Only one sample, Sample 530A-21-4, 70-71 cm (310.7 m), of the $22 \mathrm{sam}-$ ples taken in this interval, contains a poorly preserved assemblage of C. macintyrei, Coccolithus eopelagicus, C. pelagicus, $D$. bollii, $D$. exilis, $D$. kugleri, $D$. variabilis, $R$. pseudoumbilica, S. abies, and Triquetrorhabdulus rugosus, suggesting the $D$. exilis Zone (NN6 to NN7, CN5, WPN22).

The $S$. heteromorphus Zone (NN5, CN4, WPN21) is found from Section 21-6 (323.0 m) down to at least 24-2 $(345.2 \mathrm{~m})$, and may extend farther down, but the section 
is barren through $31-1(410.7 \mathrm{~m})$. The interval from Sections $31-2$ to $34-6(412.3 \mathrm{~m}$ to $446.7 \mathrm{~m})$ is largely barren, but a few samples contain moderately well-preserved assemblages indicating the Dictyococcites bisectus Subzone of the Sphenolithus ciperoensis Zone (NP25, CP19b, WPN17b). This is underlain by a short, but better-represented, interval of the Cyclicargolithus floridanus Subzone (NP24, CP19a, WPN17a) from 35-1 to $37-1$ ( $448.7 \mathrm{~m}$ to $467.0 \mathrm{~m})$.

Core $37(467.0$ to $476.5 \mathrm{~m})$ contains a relatively wellrepresented, unusually compressed section. The late Oligocene $C$. floridanus Subzone (NP24, CP19a, WPN17a) is found in Sample 530A-37-1, 1-2 cm. From 37-1, $63-64 \mathrm{~cm}$ to $37-2,2-3 \mathrm{~cm}$, the $S$. distentus Zone, S. predistentus Zone, and $R$. hillae Subzone (NP23 to NP22, CP18 to CP16c, WPN16 to WPN14c) are indicated. This interval is characterized by the Braarudosphaera bigelowi ooze, which has been reported at DSDP Holes 14, $17,19,20,22,362 \mathrm{~A}$, and 363 in the South Atlantic Ocean (Maxwell et al., 1970; Bolli et al., 1978). This is a particularly thin section of Braarudosphaera ooze, and is likely an erosional remnant of a much thicker section. It is bounded on either end by barren intervals of unknown duration, and nearby Holes $362 \mathrm{~A}$ and 363 on the Walvis Ridge contain as many as $42 \mathrm{~m}$ of Braarudosphaera ooze or chalk (Bolli et al., 1978).

Samples 37-2, 52-53 cm and 37-2, 81-82 cm are earliest Oligocene (NP21, CP16a and 16b, WPN14a and 14b) and are bounded by the last occurrence of Calcidiscus formosus and Discoaster saipanensis on the top and bottom, respectively. The latest Eocene, Isthmolithus recurvus Subzone (NP19/20, CP15b, WPN13b) is indicated by the assemblage in Sample $37-2,87-88 \mathrm{~cm}$. Present in rare to few numbers are $R$. dictyoda, $I$. recurvus, $D$. saipanensis, $D$. tanii, and $R$. umbilica. Martini's (1971) D. tanii nodifer Zone (NP16, CP13/14) is suggested in Sample 530A-37-2, 105-106 cm with the lowest occurrence of D. tanii. Section 8 of Core 37 is middle Eocene (NP15, CP13b).

The early-mid Eocene D. sublodoensis Zone (NP14, CP12), bounded on the top by the first occurrence of Nannotetrina alata in 37,CC and by the first occurrence of $D$. sublodoensis in $38-2,1-2 \mathrm{~cm}$, is found between 476.5 and $478.0 \mathrm{~m}$ sub-bottom. The early Eocene (NP13 to NP10, CP11 to CP9) is well defined from $38-2$, $133-134 \mathrm{~cm}$ to $41-1,75-76 \mathrm{~cm}(479.3 \mathrm{~m}$ to $505.8 \mathrm{~m})$. The D. lodoensis Zone (NP13, CP11) is bounded on the bottom by the last occurrence of Tribrachiatus orthostylus in $39-2,19-20 \mathrm{~cm}$. The $T$. orthostylus Zone (NP12, CP10) is bounded on the bottom by the first occurrence of $D$. lodoensis in 40-3, 122-123 cm. Subzones in the $D$. diastypus Zone (NP10/11, CP9) are not resolvable; the zone is bounded on the bottom by the first occurrence of $T$. bramlettei in Sample 530A-41-1, 75-76 cm.

The top of the Paleocene, D. multiradiatus Zone (NP9, CP8), occurs from 41-1, 141-142 cm to $42-2$, $63-64 \mathrm{~cm}(506.4 \mathrm{~m}$ to $516.6 \mathrm{~m})$; its base is determined by the first occurrence of $D$. multiradiatus. The two subzones are not distinguished. The first occurrences of both $D$. nobilis and Heliolithus riedeli in 43,CC (533.5 m) established the base of the $H$. riedeli Zone (NP8) of
Martini (1971), and the base of the D. nobilis Zone (CP7) of Bukry (1973). H. riedeli is usually not used because of its rare and sporadic occurrence in oceanic assemblages (Bukry, 1973), but in this case both zonal markers occur at the same level.

The D. mohleri Zone (NP7, CP6) is short, restricted to only one section, Section 44-1 (533.5 to $535.0 \mathrm{~m})$. Its base is defined by the first occurrence of $D$. mohleri.

The $H$. kleinpellii Zone (NP6, CP5) extends from $42-2,75-76 \mathrm{~cm}$ to $46, \mathrm{CC}(535.7$ to $562.0 \mathrm{~m})$, the first occurrence of $H$. kleinpellii. The Fasciculithus tympaniformis Zone (NP5, CP4) is present in only two samples, $47-1,18-19 \mathrm{~cm}$ and $47-1,70-71 \mathrm{~cm}$. The first occurrence of $F$. involutus in the lower sample defines the base of this zone.

The Ellipsolithus macellus Zone (NP4, CP3) is not distinguished and may be missing. Assemblages of the Chiasmolithus danicus Zone (NP3, CP2) and the Cruciplacolithus tenuis Zone (NP2) (Martini, 1971), or Subzone (CP1b) (Bukry, 1973), are found mixed together from $47-1,143-144 \mathrm{~cm}$ to $48, C C$ (563.4 to $581.0 \mathrm{~m}$ ). Chiasmolithus danicus, the first occurrence of which separates these two zones, is found only in the first of ten samples downcore. Poor preservation obscures the true first occurrence datum. Core 49 and part of Core $50(581.0$ to $590.7 \mathrm{~m})$, down to $50-1,16-17 \mathrm{~cm}$, the first occurrence of Cruciplacolithus tenuis, are in the C. tenuis Zone.

The lowermost Paleogene, or Danian (NP1, CP1a), occurs in a $142 \mathrm{~cm}$ interval from $50-1,30-31 \mathrm{~cm}$ down to $50-2,22-23 \mathrm{~cm}$. The bottom of the Paleogene, or the Cretaceous/Tertiary boundary, is defined here by the first occurrence of Biantholithus sparsus (592.2 m subbottom).

Obtaining a section across the Cretaceous/Tertiary boundary was one of the objectives of Leg 75 . The boundary is well represented in this unit, documented by poorly to moderately preserved, common to abundant nannofossils in Core 50, Section $2(592.0 \mathrm{~m})$. Table $2 \mathrm{H}$ shows the occurrences of nannofossils at intervals sampled close to the boundary. They reveal that the boundary, as far as can be judged by calcareous nannoplankton, lies between 23 and $28 \mathrm{~cm}$ in Core 50, Section 2. The high relative abundance of Micula staurophora across the boundary is a reflection of both the dissolution resistance of $M$. staurophora (Thierstein, 1980), as well as the generally poor preservational record of the other nannofossils in the assemblage (Thierstein, 1981). The boundary is not a sharp break between Maestrichtian and Paleocene assemblages, and there may be some interlayering or mixing. Paleomagnetic studies (Keating, this volume) show a shift in polarity just below the paleontologic boundary, from normal above to reversed below $63 \mathrm{~cm}$.

Hole 530B. A complete Holocene-Pleistocene record is present from the mudline to $28, \mathrm{CC}(113.2 \mathrm{~m}$ sub-bottom). The presence of the Holocene Emiliania huxleyi Zone (NN21, CN15, WPN32) was confirmed using the scanning electron microscope. A typical assemblage down to 2-1, 10-11 cm $(2.5 \mathrm{~m})$ consists of Calcidiscus leptoporus, Coccolithus carteri, C. pelagicus, Crenalith- 
us doronicoides, E. huxleyi, Gephyrocapsa caribbeanica, G. oceanica, Helicosphaera carteri, and Umbilicosphaera sibogae. Four Pleistocene subzones of Bukry (1975) and Ellis (1979) are discernable. The G. oceanica Zone of Martini (1971), or the Ceratolithus cristatus Subzone of Bukry $(1975)$ and Ellis $(1979,1982)$ (NN20, CN14b, WPN31b), extends from Samples 2-2, 113-114 $\mathrm{cm}$, below the first occurrence of $E$. huxleyi, down to $7-1,55-56 \mathrm{~cm}$ (5.0 to $24.0 \mathrm{~m}$ ), just above the last occurrence of $E$. ovata.

From the last occurrence of $E$. ovata in 7,CC (27.8 m) to the first occurrence of $G$. oceanica in $13, C C(954.2 \mathrm{~m})$, the $E$. ovata Subzone (CN14a, WPN31a) is present. Two other datums of potential biostratigraphic importance are present in this subzone: the last occurrences of Calcidiscus macintyrei in 11,CC (45.4 m) and Discolithina japonica in 13,CC.

The G. caribbeanica Subzone (mid NN19, CN13b, WPN30b) is present from $14, \mathrm{CC}$ to the first occurrence of G. caribbeanica in $18, \mathrm{CC}(58.2$ to $76.2 \mathrm{~m})$. The earliest Pleistocene E. annula Subzone (early NN19, CN13a, WPN30a) extends down to $28, C C(113.2 \mathrm{~m})$. The last occurrences of $D$. brouweri and D. variabilis in $29, C$ C $(116.2 \mathrm{~m})$ indicate that the Pleistocene lies unconformably upon the earliest late Pliocene Discoaster tamalis Subzone (lower NN16, CN12a, WPN29a). From 30,CC to $40, \mathrm{CC}(118.2$ to $154.6 \mathrm{~m})$ the $D$. asymmetricus Subzone (upper NN15, CN11b, WPN28b) is indicated, bounded on top by the last occurrence of Sphenolithus abies and on the bottom by the first occurrence of $D$. $t a$ malis. Below this, the $S$. neoabies Subzone (lower NN15, CN11a, WPN28a) continues from $41, \mathrm{CC}$ to $44, \mathrm{CC}$ (158.0 to $167.5 \mathrm{~m}$ ) and lies unconformably upon the late Miocene.

The top of the late Miocene begins between 44,CC and $45, C C$ with the last occurrence of $D$. berggrenii and $D$. quinqueramus in $45, \mathrm{CC}(170.8 \mathrm{~m})$. This defines the top of the Amaurolithus primus Subzone (upper NN11, CN9b, WPN26b) which continues to the bottom of the cored section in $48, \mathrm{CC}(180.6 \mathrm{~m})$.

\section{Site 531}

Site $531\left(19^{\circ} 38.40^{\prime} \mathrm{S}, 9^{\circ} 35.47^{\prime} \mathrm{E}\right.$; water depth, 1267 $\mathrm{m}$ ) is located on the eastern end of the Walvis Ridge. Two holes were attempted at Site 531, but in each case the bottom was found to be firm and could not be penetrated. Hole 531 yielded only a core catcher filled with foraminiferal ooze. Hole 531A was 1000 feet west and yielded only $27 \mathrm{~cm}$ of foraminiferal ooze.

Holes 531/531A. The following well-preserved, abundant calcareous nannofossils, typically found in the Holocene Emiliania huxleyi Zone (NN21, CN15, WPN32), were observed in 531-1,CC (1284 m): Calcidiscus leptoporus, Coccolithus pelagicus, E. huxleyi, Geophyrocapsa oceanica, Helicosphaera carteri, Pontosphaera syracusana, Syracosphaera pulchra, and Umbilicosphaera mirabilis. In addition to these species, the following occur in Section 531A-1-1: Ceratolithus cristatus, Rhabdosphaera clavigera, and Scapholithus fossilis.

\section{Site $\mathbf{5 3 2}$}

Site $532\left(19^{\circ} 41.61^{\prime} \mathrm{S}, 10^{\circ} 31.13^{\prime} \mathrm{E}\right.$; water depth, 1331 $\mathrm{m}$ ) is located on the eastern part of the Walvis Ridge in a trough with relatively thick sediment fill, at a position close to Site 362 from Leg 40 . The uppermost section at Site 362 was badly disturbed by rotary coring, and the HPC was employed at Site 532 in an attempt to recover an undisturbed upper section, close to the Walvis Bay zone of upwelling and high biotic productivity. Three holes were hydraulic piston cored (HPC) at Site 532. Hole 532 yielded 61 cores reaching a depth of $250.8 \mathrm{~m}$. Hole $532 \mathrm{~A}$ was offset about $50 \mathrm{~m}$ to the SSW. Fortyseven cores were taken to reach a depth of $199.6 \mathrm{~m}$. This set of cores was taken for the JOIDES Sedimentary Petrology and Physical Properties Panel; the cores were sealed and not opened for examination and sampling aboard ship. Hole 532B was offset about $30 \mathrm{~m}$ to the south. Seventy-four cores were taken, reaching a depth of $291.3 \mathrm{~m}$. Cores 1 to 56 were not opened, but were frozen to be examined later for geochemical studies. The remainder of the cores were studied in the usual manner. Control from the rig floor was so exact, that Cores 532-61 and 532B-61 correlate within $0.4 \mathrm{~m}$ and can be considered to have been taken at the same level. Hence, coring is continuous from the mudline to the total depth of $291.3 \mathrm{~m}$ in Core 532B-74.

A thick $(291.3 \mathrm{~m})$ Holocene to late Miocene unit of nannofossil marl and ooze was encountered at Site 532 . The section was divided into three subunits: $1 \mathrm{a}$, foramnannofossil marl and ooze (Cores 1-12, 0 to $49.5 \mathrm{~m}$, Pleistocene), 1b, diatom-nannofossil marl (Cores 12$26,49.5$ to $114.0 \mathrm{~m}$, late Pliocene), and $1 \mathrm{c}$, nannofossil marl (Cores 27-74, 114.0 to $291.3 \mathrm{~m}$, late Pliocene to late Miocene). Calcareous nannofossils are common to abundant, showing moderate to good preservation in all samples studied. No barren intervals were encountered.

Figure 2 shows a comparison between the calcareous nannofossil biostratigraphy of Hole 362 (Bukry, 1978; Proto Decima, 1978) and Holes 532 and 532B. Immediately evident is the fact that the epoch boundaries in both sections are within $10 \mathrm{~m}$ of each other. The zonal boundaries, however, do not correlate as well. The higher zonal resolution in Holes 532 and 532B is the result of the diminished core disturbance with the HPC used in obtaining that core. The low resolution in the Early Pliocene sections of both Holes 362 and 532/532B is likely the result of slumping in the sediment.

Holes 532 and 532B. The Holocene Emiliania huxleyi Zone is detected from the mudline to Sample 1-2, 62-63 $\mathrm{cm}$ ( $2.1 \mathrm{~m}$ sub-bottom). A complete Pleistocene section, including the four subzones of Bukry (1975) and Ellis $(1979,1982)$, is present from $1-3,65-66 \mathrm{~cm}$ down to $17, \mathrm{CC}(74.4 \mathrm{~m})$. The $E$. ovata datum occurs in 5,CC $(21.6 \mathrm{~m})$; the Gephyrocapsa oceanica datum occurs in $11, \mathrm{CC}(48.0 \mathrm{~m})$; and the $G$. caribbeanica datum occurs in $15, C C(65.6 \mathrm{~m})$.

The last occurrence of Discoaster brouweri, defining the Pliocene/Pleistocene boundary, occurs in 18-1, 

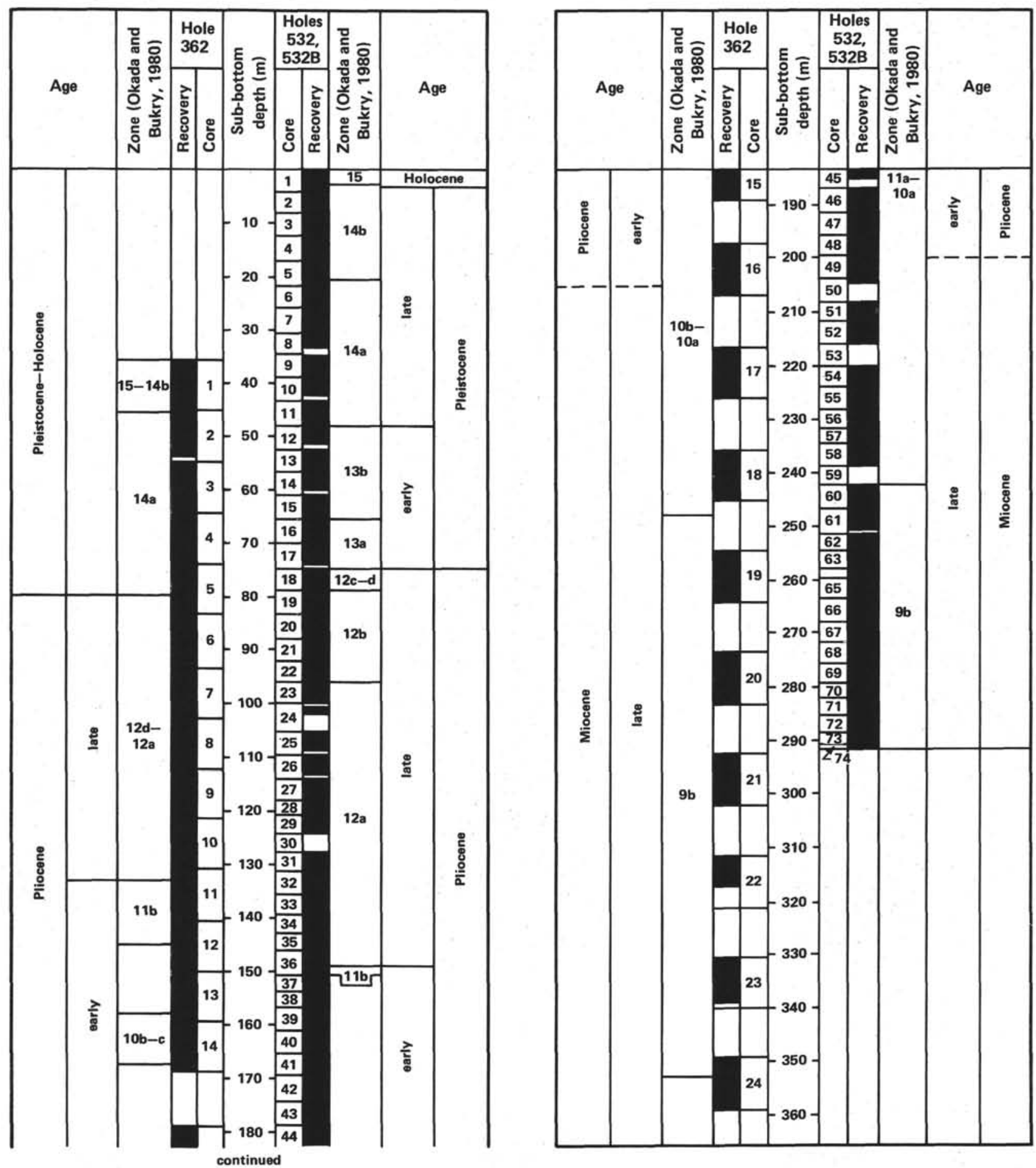

Figure 2. Comparison between the calcareous nannofossil biostratigraphy of Hole 362 and Holes 532 and 532B. Nannofossil zones and subzones are numbered according to the scheme proposed by Okada and Bukry (1980) and shown in Table 6.

$125-126 \mathrm{~cm}(75.6 \mathrm{~m})$. Three of four late Pliocene subzones of Bukry $(1973,1975)$ and Ellis $(1979,1982)$ are detectable; the upper two appear to be mixed together from $18-1,125-126 \mathrm{~cm}$ to $18-3,121-122 \mathrm{~cm}$. The last occurrences of both $D$. pentaradiatus and $D$. surculus in $18, C C(78.8 \mathrm{~m})$ indicate the top of the D. surculus Subzone (CN12b, WPN29b). This subzone extends down to $22-3,80-81 \mathrm{~cm}(95.8 \mathrm{~m})$. The last occurrence of $D$. $t a-$ malis in 22, CC $(96.4 \mathrm{~m})$ indicates the top of the $D$. tamalis Subzone. This extends down to $36-2,107-108 \mathrm{~cm}$ (149.1 m).

Ellis' (1982) D. asymmetricus Subzone (WPN28b) is found in only one sample examined: $36, C \mathrm{C}(150.6 \mathrm{~m})$. This sample contains the two datums defining this subzone: the first occurrence of $D$. tamalis and the last occurrence of Sphenolithus abies. Bukry's $(1973,1975) D$. 
asymmetricus Subzone (CN11b) is defined on the bottom of the acme of D. asymmetricus. This is not detectable in Hole 532.

The zones or subzones of the early Pliocene are not resolvable. From $37, \mathrm{CC}$ to $60-3,31-32 \mathrm{~cm}$ (153.8 to $241.1 \mathrm{~m}$ ), an early Pliocene assemblage (NN12-NN15, CN10a-CN11a, WPN27a-WPN28a) is present, but none of the datums is present in any meaningful order.

The late Miocene Amaurolithus primus Subzone (upper NN11, CN9b, WPN26b) occurs from $60, \mathrm{CC}$ to the bottom of the cored interval, 74B,CC (246.8 to $291.3 \mathrm{~m}$ ). The first occurrence of $A$. primus, defining the bottom of this subzone, was not reached.

\section{TAXONOMY}

Biantholithus astralis Steinmetz and Stradner nov. spec. (Plate 52, Figs. 1, 4; Plate 53, Figs. 1, 2)

Description: This new species consists of seven to eight segments radiating from a common center. The segments are slightly imbricate and slightly tilted, as are the blades of a windmill. Each segment is in contact with adjacent segments for a little more than half its length. Beyond the point of contact, the segments taper slightly and end with rounded tips. A small hole is present in the center where the segments meet.

Remarks: Biantholithus sparsus Bramlette and Martini differs from Biantholithus astralis nov. spec. in that it has eight to twelve segments which are in contact for almost their entire length.

Holotype: Plate 53, Figure 1. Negative 2/92/007, Elmi-Lab, Geological Survey of Austria, Vienna.

Type locality and level: Southeast Atlantic Ocean, Angola Basin, DSDP 530A-50-1, 6-7 cm, Cruciplacolithus tenuis Zone (Martini, 1971), Cruciplacolithus tenuis Subzone (Bukry, 1973, 1975).

Known range: Danian.

\section{ACKNOWLEDGMENTS}

We thank Anthony Greco and Gladys Swigert of the Scanning Electron Microscope Facility, University of South Florida, and Franz Allram of the Elmi-Lab, Geological Survey of Austria, Vienna, for their assistance with the scanning electron microscopy. The SEM at the University of South Florida was purchased in part by NSF grant OCE 79-13233.

We are grateful to William $\mathrm{H}$. Lohman and John L. Wray for their critical review of the manuscript.

\section{REFERENCES}

Bolli, H. M., Ryan, W. B. F., et al., 1978. Init. Repts. DSDP, 40: Washington (U.S. Govt. Printing Office).

Bramlette, M. N., and Sullivan, F. R., 1961. Coccolithophorids and related nannoplankton of the early Tertiary in California. Micropaleontology, 7:129-174.

Bukry, D., 1973. Coccolith stratigraphy, Eastern Equatorial Pacific, Leg 16, Deep Sea Drilling Project. In van Andel, Tj. H., Heath, G. R., et al., Init. Repts. DSDP, 16: Washington (U.S. Govt. Printing Office), 653-712.

1975. Coccolith and silicoflagellate stratigraphy, northwestern Pacific Ocean, Deep Sea Drilling Project Leg 32. In Larson, R. L., Moberly, R., et al., Init. Repts. DSDP, 32: Washington (U.S. Govt. Printing Office), 677-701.

1978. Cenozoic silicoflagellate and coccolith stratigraphy, southeastern Atlantic Ocean, Deep Sea Drilling Project Leg 40. In Bolli, H. M., Ryan, W. B. F., et al., Init. Repts. DSDP, 40. Washington (U.S. Govt. Printing Office), 635-649.

Ellis, C. H., 1979. Neogene nannoplankton zonation in eastern Mediterranean. Proc. 7th Int. Cong. Med. Neogene (Tome hors serie), $1: 391-401$.

1982. Calcareous nannoplankton biostratigraphy-Deep Sea Drilling Project Leg 60. In Hussong, D. M., Uyeda, S., et al., Init. Repts. DSDP, 60: Washington (U.S. Govt. Printing Office), 507-535.

Hay, W. W., 1961. Note on the preparation of samples for discoasterids. J. Paleontol., 35:873.
1965. Calcareous nannofossils. In Kummel, B., and Raup, D. (Eds.), Handbook of Paleontological Techniques: San Francisco (W. H. Freeman and Co.), pp. 3-7.

1970. Calcareous nannofossils from cores recovered on DSDP Leg 4. In Bader, R. G., Gerard, R. D., et al., Init. Repts. DSDP, 4: Washington (U.S. Govt. Printing Office), 455-503.

Martini, E., 1971. Standard Tertiary and Quaternary calcareous nannoplankton zonation. In Farinacci, A. (Ed.), Proc. Second Plankt. Conf., Roma, 1970: Rome (Tecnoscienza), 2:739-785.

Maxwell, A. E., Von Herzen, R. P., et al., 1970. Init. Repts. DSDP, 3: Washington (U.S. Govt. Printing Office).

Okada, H., and Bukry, D., 1980. Supplementary modification and introduction of code numbers to the low-latitude coccolith biostratigraphic zonation (Bukry, 1973; 1975). Mar. Micropaleontol, 5: 321-325.

Proto Decima, F. Medizza, F., and Todesco, L., 1978. Southeastern Atlantic Leg 40 calcareous nannofossils. In Bolli, H. M., Ryan, W. B. F., et al., Init. Repts. DSDP, 40: Washington (U.S. Govt. Printing Office), 571-634.

Steinmetz, J. C., 1979. Calcareous nannofossils from the North Atlantic Ocean, Leg 49, Deep Sea Drilling Project. In Luyendyk, B. P., Cann, J. R., et al., Init. Repts. DSDP, 49: Washington (U.S. Govt. Printing Office), 519-531.

Thierstein, H. R., 1980. Selective dissolution of Late Cretaceous and earliest Tertiary calcareous nannofossils: Experimental evidence. Cret. Res., 2:165-176.

1981. Late Cretaceous nannoplankton and the change at the Cretaceous-Tertiary boundary. In Warme, J. E., Douglas, R. G., and Winterer, E. L. (Eds.), The Deep Sea Drilling Project: $A$ Decade of Progress. Soc. Econ. Paleont. Mineral, Spec. Publ., 32: 355-394.

Date of Initial Receipt: November 9, 1982

APPENDIX

Cenozoic Calcareous Nannofossils Considered in This Chapter and Index to Plates (in alphabetical order by species epithets)

\begin{tabular}{|c|c|}
\hline & Plate: Figures \\
\hline Sphenolithus abies Deflandre, 1954 & $41: 1,2,9,10$ \\
\hline $\begin{array}{l}\text { Cyclicargolithus abisectus (Müller, 1970) } \\
\text { Bukry, } 1973\end{array}$ & $28: 3$ \\
\hline $\begin{array}{l}\text { Ceratolithus acutus Gartner and Bukry, } \\
\text { Calcidiscus aequiscutum (Gartner, 1967) } \\
\text { Loeblich and Tappan, } 1978\end{array}$ & $24: 1-6$ \\
\hline $\begin{array}{l}\text { Nannotetrina alata (Martini, 1960) Haq and } \\
\quad \text { Lohmann, } 1976\end{array}$ & \\
\hline Pontosphaera alboranensis Bartolini, 1970 & $7: 1,2$ \\
\hline $\begin{array}{l}\text { Chiasmolithus altus Bukry and Percival, } 1971 \\
\text { Discoaster altus } \text { Müller, } 1974\end{array}$ & $29: 1-4$ \\
\hline \multicolumn{2}{|l|}{$\begin{array}{l}\text { Helicosphaera ampliaperta } \text { Bramlette and } \\
\text { Wilcoxon, } 1967\end{array}$} \\
\hline \multicolumn{2}{|l|}{$\begin{array}{l}\text { Amaurolithus amplificus (Bukry and Percival, } \\
\text { 1971) Gartner and Bukry, } 1975\end{array}$} \\
\hline \multicolumn{2}{|l|}{$\begin{array}{l}\text { Emiliania annula (Cohen, 1964) Bukry, } 1973 \\
\text { Oolithotus antillarum (Cohen, 1964) }\end{array}$} \\
\hline Reinhardt, 1968 & \\
\hline \multicolumn{2}{|l|}{ Gephyrocapsa aperta Kamptner, 1963} \\
\hline \multicolumn{2}{|l|}{ Scyphosphaera apsteini Lohmann, 1902} \\
\hline \multicolumn{2}{|l|}{ Discoaster araneus Bukry, 1971} \\
\hline \multicolumn{2}{|l|}{ Ceratolithus armatus Müller, 1974} \\
\hline Biantholithus astralis nov. spec. & $52 ; 1,4 ; 53: 1,2$ \\
\hline $\begin{array}{l}\text { Markalius astroporus (Stradner, 1963) Hay and } \\
\text { Mohler, } 1967\end{array}$ & $\begin{array}{l}45: 6 ; 50: 1-4 ; \\
\quad 51: 1,2,5-7\end{array}$ \\
\hline Discoaster asymmetricus Gartner, 1969 & $15: 1-4,6$ \\
\hline \multicolumn{2}{|l|}{ Discoaster aulakos Gartner, 1967} \\
\hline Discoaster barbadiensis Tan Sin Hok, 1927 & $30: 2$ \\
\hline \multicolumn{2}{|l|}{$\begin{array}{l}\text { Sphenolithus belemnos Bramlette and } \\
\text { Wilcoxon, } 1967\end{array}$} \\
\hline Discoaster bellus Bukry and Percival, 1971 & \\
\hline Discoaster berggrenii Bukry, 1971 & $18: 4$ \\
\hline $\begin{array}{l}\text { Chiasmolithus bidens (Bramlette and Sullivan, } \\
\text { 1961) Hay and Mohler, } 1967\end{array}$ & \\
\hline
\end{tabular}


Appendix. (Continued).

Plate: Figures

Braarudosphaera bigelowi (Gran and Braarud, 21:3; 25:1-4 1935) Deflandre, 1947

Zygrhablithus bijugatus (Deflandre, 1954) Deflandre, 1959

Discoaster binodosus Martini, 1958

Reticulofenestra bisecta (Hay, Mohler, and Wade, 1966) Roth, 1973

Prinsius bisulcus (Stradner, 1963) Hay and Mohler, 1967

Discoaster blackstockae Bukry, 1973

Discoaster bollii Martini and Bramlette, 1963

Discoaster braarudii Bukry, 1971

Tribrachiatus bramlettei (Brönnimann and Stradner, 1960) Proto Decima, Roth, and Todesco, 1975

Discoaster brouweri Tan Sin Hok, 1927

Discoaster calcaris Gartner, 1967

Discoaster calculosus Bukry, 1971

Catinaster calyculus Martini and Bramlette, 1963

Heliolithus cantabriae Perch-Nielsen, 1971

Gephyrocapsa caribbeanica Boudreaux and Hay, 1967

Triquetrorhabdulus carinatus Martini, 1965

Coccolithus carteri (Wallich, 1877) Kamptner, 1941

Helicosphaera carteri (Wallich, 1877)

Kamptner, 1954

Ericsonia cava (Hay and Mohler, 1967) PerchNielsen, 1969

Discoaster challengeri Bramlette and Riedel, 1954

Neochiastozygus chiastus (Bramlette and Sullivan, 1961) Perch-Nielsen, 1971

Sphenolithus ciperoensis Bramlette and Wilcoxon, 1967

Rhabdosphaera clavigera Murray and Blackman, 1898

Catinaster coalitus Martini and Bramlette, 1963

Discoaster colleti (Parejas, 1934) Bersier, 1939

Helicosphaera compacta Bramlette and Wilcoxon, 1967

Chiasmolithus consuetus (Bramlette and Sullivan, 1961) Hay and Mohler, 1967

Tribrachiatus contortus (Stradner, 1958) Bukry, 1972

Minilitha convallis Bukry, 1973

Coccolithus crassus Bramlette and Sullivan, 1961

Toweius craticulus Hay and Mohler, 1967

Coccolithus cribellum (Bramlette and Sullivan, 1961) Stradner, 1962

Ceratolithus cristatus Kamptner, 1954

Nannotetrina cristata (Martini, 1958) PerchNielsen, 1971

Rhomboaster cuspis Bramlette and Sullivan, 1961

Chiasmolithus danicus (Brotzen, 1959) Hay and Mohler, 1967

Discoaster decorus (Bukry, 1971) Bukry, 1973

Discoaster deflandrei Bramlette and Riedel, 1954

Thoracosphaera deflandrei Kamptner, 1956

Campylosphaera dela (Bramlette and Sullivan, 1961) Hay and Mohler, 1967

Amaurolithus delicatus Gartner and Bukry, 1975

Discoaster delicatus Bramlette and Sullivan, 1961

$32: 1,2 ; 33: 2-5$

28:9, 10

$23: 8$

$12: 1-4 ; 19: 1,2$ $21: 1,2$

45:1-2

$1: 2-4 ; 6: 3$

$3: 1,3,5-7$

9:1-4

$43: 6 ; 44: 1,2$

$17: 2$

$8: 1-5$

$23: 4,5$

$32: 3,4 ; 33: 6-9$

$42: 1,2$

$45: 3$

$22: 3$

$17: 4 ; 36: 1$

$10: 1,2 ; 11: 1$
Appendix. (Continued).

Plate: Figures

Sphenolithus delphix Bukry, 1973

Discoaster diastypus Bramlette and Sullivan, 1961

Dictyococcites dictyoda (Deflandre and Fert, 1954) Martini, 1959

Discolithina discopora (Schiller, 1925)

Braarudosphaera discula Bramlette and Riedel, 1954

Sphenolithus distentus (Martini, 1965)

Bramlette and Wilcoxon, 1967

Ellipsolithus distichus (Bramlette and Sullivan, 1961) Sullivan, 1964

Discoaster distinctus Martini, 1958

Crenalithus doronicoides (Black and Barnes, 1961) Roth, 1973

Neococcolithites dubius (Deflandre, 1954) Black, 1967

Discoaster druggii Bramlette and Wilcoxon, 1967

Cruciplacolithus edwardsii Romein, 1979

Toweius eminens (Bramlette and Sullivan, 1961) Perch-Nielsen, 1971

Coccolithus eopelagicus (Bramlette and Riedel, 1954) Bramlette and Sullivan, 1961

Helicosphaera euphratis Haq, 1966

Discoaster exilis Martini and Bramlette, 1963

Chiasmolithus expansus (Bramlette and Sullivan, 1961) Gartner, 1970

Discoaster falcatus Bramlette and Sullivan, 1961

Heliorthus fallax Brönnimann and Stradner, 1960

Ericsonia fenestrata (Deflandre and Fert, 1954) Stradner, 1968

Cyclicargolithus floridanus (Roth and Hay, 1967) Bukry, 1971

Micrantholithus flos Deflandre, 1954

Calcidiscus formosus (Kamptner, 1963) Loeblich and Tappan, 1978

Discoaster formosus Martini and Worsley, 1971

Scapholithus fossilis Deflandre, 1954

Nannotetrina fulgens (Stradner, 1960) Stradner, 1969

Calcidiscus gammation (Bramlette and Sullivan, 1961) Loeblich and Tappan, 1978

Reticulofenestra gartneri Roth and Hay, 1967

Chiasmolithus gigas (Bramlette and Sullivan, 1961) Radomski, 1968

Chiasmolithus grandis (Bramlette and Riedel, 1954) Radomski, 1968

Discoaster hamatus Martini and Bramlette, 1963

Thoracosphaera heimii (Lohmann, 1919) Kamptner, 1954

Discoaster helianthus Bramlette and Sullivan, 1961

Sphenolithus heteromorphus Deflandre, 1953

Reticulofenestra hillae Bukry and Percival, 1971

Syracosphaera histrica Kamptner, 1941

Emiliania huxleyi (Lohmann, 1902) Hay and Mohler, 1967

Discoaster icarus Stradner, 1973

Thoracosphaera imperforata Kamptner, 1952

Discoaster intercalcaris Bukry, 1971

$41: 3,4$

$46: 4$

$36: 2$

$2: 3,4 ; 6: 6$

$47: 5$

$43: 4 ; 44: 3,4$

$17: 1,20: 3 ; 23: 9$

$28: 1,2$

42:7

Discosphaera intermedia Deflandre, 1942

Scyphosphaera intermedia Deflandre, 1942

Markalius inversus (Deflandre, 1954) Bramlette and Martini, 1964

Triquetrorhabdulus inversus Bukry and Bramlette, 1969 
Appendix. (Continued).

Plate: Figures

Fasciculithus involutus Bramlette and Sullivan, 41:7, 8 1961

Discolithina japonica Takayama, 1967

Cricolithus jonesii Cohen, 1965

Neochiastozygus junctus (Bramlette and

Sullivan, 1961) Perch-Nielsen, 1971

Heliolithus kleinpellii Sullivan, 1964

Discoasteroides kuepperi (Stradner, 1959) Bramlette and Sullivan, 1961

Discoaster kugleri Martini and Bramlette, 1963

Discoaster lenticularis Bramlette and Sullivan, 1961

Calcidiscus leptoporus (Murray and Blackman, 1898) Loeblich and Tappan, 1978

Discoaster lodoensis Bramlette and Riedel, 1954

Discoaster loeblichii Bukry, 1971

Helicosphaera lophota (Bramlette and Sullivan, 1961) Jafar and Martini, 1975

Ellipsolithus macellus (Bramlette and Sullivan, 1961) Sullivan, 1964

Calcidiscus macintyrei (Bukry and Bramlette, 1969) Loeblich and Tappan, 1978

Coccolithus magnicrassus Bukry, 1971

Discoaster mediosus Bramlette and Sullivan, 1961

Discoaster megastypus Bramlette and Sullivan, 1961

Triquetrorhabdulus milowii Bukry, 1971

Lanternithus minutus Stradner, 1962

Coccolithus miopelagicus Bukry, 1971

Umbilicosphaera mirabilis Lohmann, 1902

Discoaster mirus Deflandre, 1954

Discoaster mohleri Bukry and Percival, 1971

Sphenolithus moriformis (Brönnimann and Stradner, 1960) Bramlette and Wilcoxon, 1967

Discolithina multipora (Kamptner, 1948) Martini, 1965

Discoaster multiradiatus Bramlette and Riedel, 1954

Discoaster musicus Stradner, 1959

Sphenolithus neoabies Bukry and Bramlette, 1969

Discoaster neohamatus Bukry and Bramlette, 1969

Discoaster neorectus Bukry, 1971

Coronocyclus nitescens (Kamptner, 1964)

Bramlette and Wilcoxon, 1967

Discoaster nobilis Martini, 1961

Chiasmolithus oamaruensis (Deflandre, 1954) Hay, Mohler, and Wade, 1966

Reticulofenestra oamaruensis (Deflandre, 1954) Stradner, 1968

Discoaster obtusus Gartner, 1967

Gephyrocapsa oceanica Kamptner, 1943

Discoaster okadai Bukry, 1981

Thoracosphaera operculata Bramlette and Martini, 1964

Coccolithus orangensis Bukry, 1971

Discoaster ornatus Stradner, 1958

Tribrachiatus orthostylus Shamrai, 1963

Emiliani ovata Bukry, 1973

Discoaster pansus (Bukry and Percival, 1971) Bukry, 1973

Nannotetrina pappii (Stradner, 1959) PerchNielsen, 1971

Coccolithus pelagicus (Wallich, 1877) Schiller, $\quad 3: 2,4$ 1930
$48: 4$

$34: 5$

$31: 3,4$

$18: 3 ; 23: 6,7$

$34: 7,8$

$4: 1,3$

$31: 2$

$34: 1-4,6 ; 46: 1-3$

$4: 2,4 ; 5: 1-4$ $10: 3,4$

$40: 4$

$34: 9 ; 35: 2-4$

$23: 2,3$

$1: 1$

$38: 1-6 ; 39: 1-4$ $40: 1-3$

$45: 7,8$

$28: 4-6$

$31: 1$

20:7-9
Appendix. (Continued).

Plate: Figures

Discoaster pentaradiatus Tan Sin Hok, 1927

$15: 5$

Blackites perlongus (Deflandre, 1954) Shafik, 1981

Hayaster perplexus (Bramlette and Riedel, 1954) Bukry, 1973

Fasciculithus pileatus Bukry, 1973

Pontosphaera plana (Bramlette and Sullivan, 1961) Haq, 1971

Zygodiscus plectopons Bramlette and Sullivan, 1961

Sphenolithus predistentus Bramlette and Wilcoxon, 1967

Discoaster prepentaradiatus Bukry and Percival, 1971

Amaurolithus primus (Bukry and Bramlette, 1971) Gartner and Bukry, 1975

Cruciplacolithus primus Perch-Nielsen, 1977

Cyclolithella prionion (Deflandre and Fert, 1954) nov. comb. (ex Cyclolithus)

Crenalithus productellus Bukry 1975

Thoracosphaera prolata Bukry and Bramlette, 1969

Neococcolithes protenus (Bramlette and Sullivan, 1961) Perch-Nielsen, 1971

Sphenolithus pseudoradians Deflandre, 1952

Reticulofenestra pseudoumbilica (Gartner, 1967) Gartner, 1969

Discoaster pseudovariabilis Martini and Worsley, 1971

Scyphosphaera pulcherrima Deflandre, 1942

Discolithina pulchra (Deflandre, 1954) Levin, 1965

Syracosphaera pulchra Lohmann, 1902

Discoaster quadramus Bukry, 1973

Discoaster quinqueramus Gartner, 1969

Sphenolithus radians Deflandre, 1952

Isthmolithus recurvus Deflandre, 1954

Cyclagelosphaera reinhardtii Wind and Wise, 1977

Helicosphaera reticulata Bramlette and Wilcoxon, 1967

Heliolithus riedelii Bramlette and Sullivan, 1961

Cyclolithella robusta (Hay, Mohler, and Wade, 1966) Bukry and Percival, 1971

Biscutum romeinii Perch-Nielsen, 1981

Braarudosphaera rosa Levin and Joerger, 1967

Calcidiscus rotula (Kamptner, 1956) Loeblich and Tappan, 1978

Ceratolithus rugosus Bukry and Bramlette, 1968

Triquetrorhabdulus rugosus Bramlette and Wilcoxon, 1967

Discoaster saipanensis Bramlette and Riedel, 1954

Discoaster salisburgensis Stradner, 1961

Thoracosphaera saxea Stradner, 1961

Pontosphaera scutellum Kamptner, 1952

Helicosphaera sellii (Bukry and Bramlette, 1969) Jafar and Martini, 1975

Helicosphaera seminulum (Bramlette and Sullivan, 1961) Jafar and Martini, 1975

Umbilicosphaera sibogae (Weber-van-Bosse, 1901) Gaarder, 1970

Rhabdosphaera sicca (Stradner, 1963) Fuchs and Stradner, 1977

Zygodiscus sigmoides Bramlette and Sullivan, 1961

Discoaster signus Bukry, 197
$47: 1,2$

43:7

$2: 1,2$

$49: 3$

$26: 1-4 ; 27: 1-4$

$22: 1,2$

$6: 1,7$

$41: 5,6$

$51: 3,4$

$42: 3-6 ; 43: 1-3$

30:1

$35: 1 ; 36: 5-7$; $45: 9$

$10: 5-7$

$7: 4$

$48: 1-3,5-7$ 
Appendix. (Continued).

Plate: Figures

Zygrhablithus simplex Bramlette and Sullivan, 1961

Biantholithus sparsus Bramlette and Martini, 1964

Discoaster splendidus Martini, 1960

Discoaster stellus Gartner, 1967

Aspidorhabdus stylifera (Lohmann, 1902)

Boudreaux and Hay, 1969

Discoaster sublodoensis Bramlette and Sullivan, 1961

Discoaster surculus Martini and Bramlette, 1963

Nannotetrina swasticoides (Martini, 1958)

Martini and Stradner, 1960

Pontosphaera syracusana Lohmann, 1902

Discoaster tamalis Kamptner, 1967

Discoaster tanii Bramlette and Riedel, 1954

Discoaster tanii nodifer Bramlette and Riedel,
Appendix. (Continued).

\begin{tabular}{|c|c|}
\hline & Plate: Figures \\
\hline $\begin{array}{l}\text { Cruciplacolithus tenuis (Stradner, 1961) Hay } \\
\text { and Mohler, } 1967\end{array}$ & $47: 3,4$ \\
\hline $\begin{array}{l}\text { Discoaster toralus Ellis, Lohmann, and Wray, } \\
1972\end{array}$ & $18: 1,2$ \\
\hline $\begin{array}{l}\text { Amaurolithus tricorniculatus (Gartner, 1967) } \\
\text { Gartner and Bukry, } 1975\end{array}$ & $11: 2,3$ \\
\hline \multicolumn{2}{|l|}{ Discoaster trinidadensis Hay, 1967} \\
\hline Discoaster trinus Stradner 1961 & $30: 4$ \\
\hline \multicolumn{2}{|l|}{ Braarudosphaera turbinea Stradner, 1963} \\
\hline \multicolumn{2}{|l|}{$\begin{array}{l}\text { Fasciculithus tympaniformis Hay and Mohler, } \\
1967\end{array}$} \\
\hline \multicolumn{2}{|l|}{$\begin{array}{l}\text { Reticulofenestra umbilica (Levin, 1965) } \\
\text { Martini and Ritzkowski, } 1968\end{array}$} \\
\hline $\begin{array}{l}\text { Discoaster variabilis Martini and Bramlette, } \\
\quad 1963\end{array}$ & $19: 8 ; 20: 4-6 ; 23: 1$ \\
\hline $\begin{array}{l}\text { Markalius variabilis } \text { Perch-Nielsen, } 1977 \\
\text { Micrantholithus vesper Deflandre, } 1954\end{array}$ & \\
\hline
\end{tabular}

Table 1. Distribution of Holocene-Pliocene calcareous nannofossils, Hole 530.

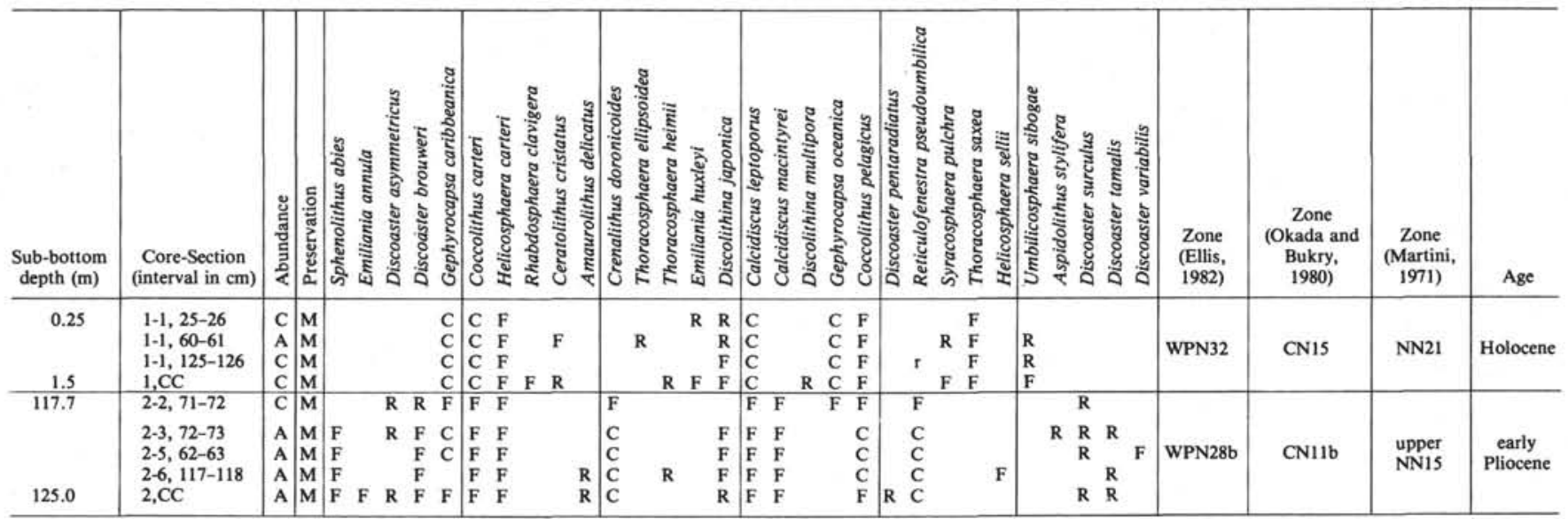

Note: Abundance: $\mathrm{A}=$ abundant $; \mathrm{C}=$ common; $\mathrm{F}=$ few; $\mathrm{R}=$ rare; $\mathrm{B}=$ barren; lower case letter = reworked older fossils. Preservation: $\mathrm{G}=\mathrm{good} ; \mathrm{M}=$ moderate; $\mathrm{P}=$ poor. 
Table 2. Distribution of calcareous nannofossils, Hole 530A 2A. Early Pliocene to Late Miocene.

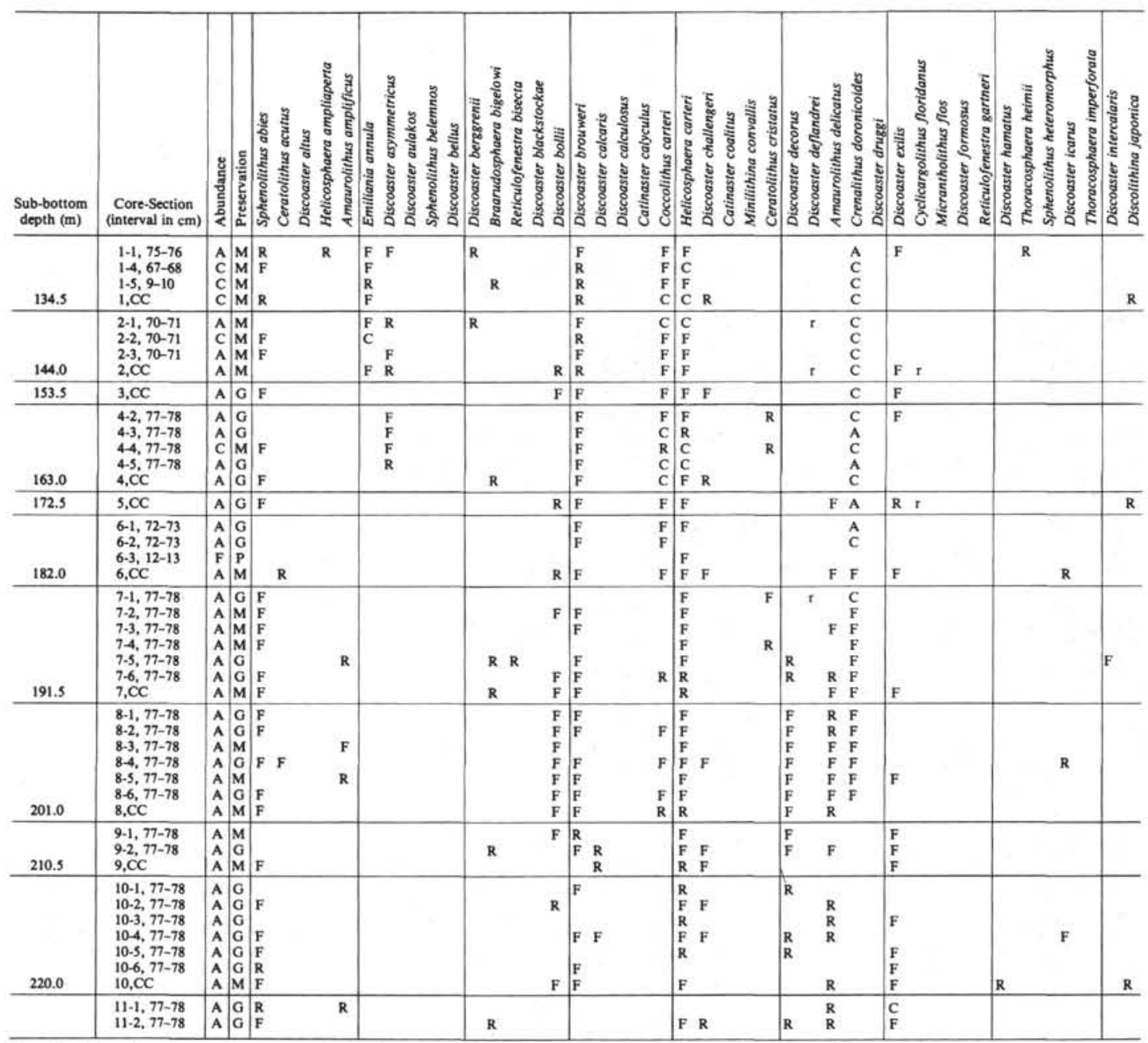

Note: Abundance: $\mathrm{A}=$ abundant; $\mathrm{C}=$ common; $\mathrm{F}=$ few; $\mathrm{R}=$ rare; $\mathrm{B}=$ barren; lower case letter $=$ reworked older fossils. Preservation: $\mathrm{G}=\mathrm{good} ; \mathrm{M}=$ moderate; $\mathrm{P}=$ poor. 
Table 2A. (Continued).

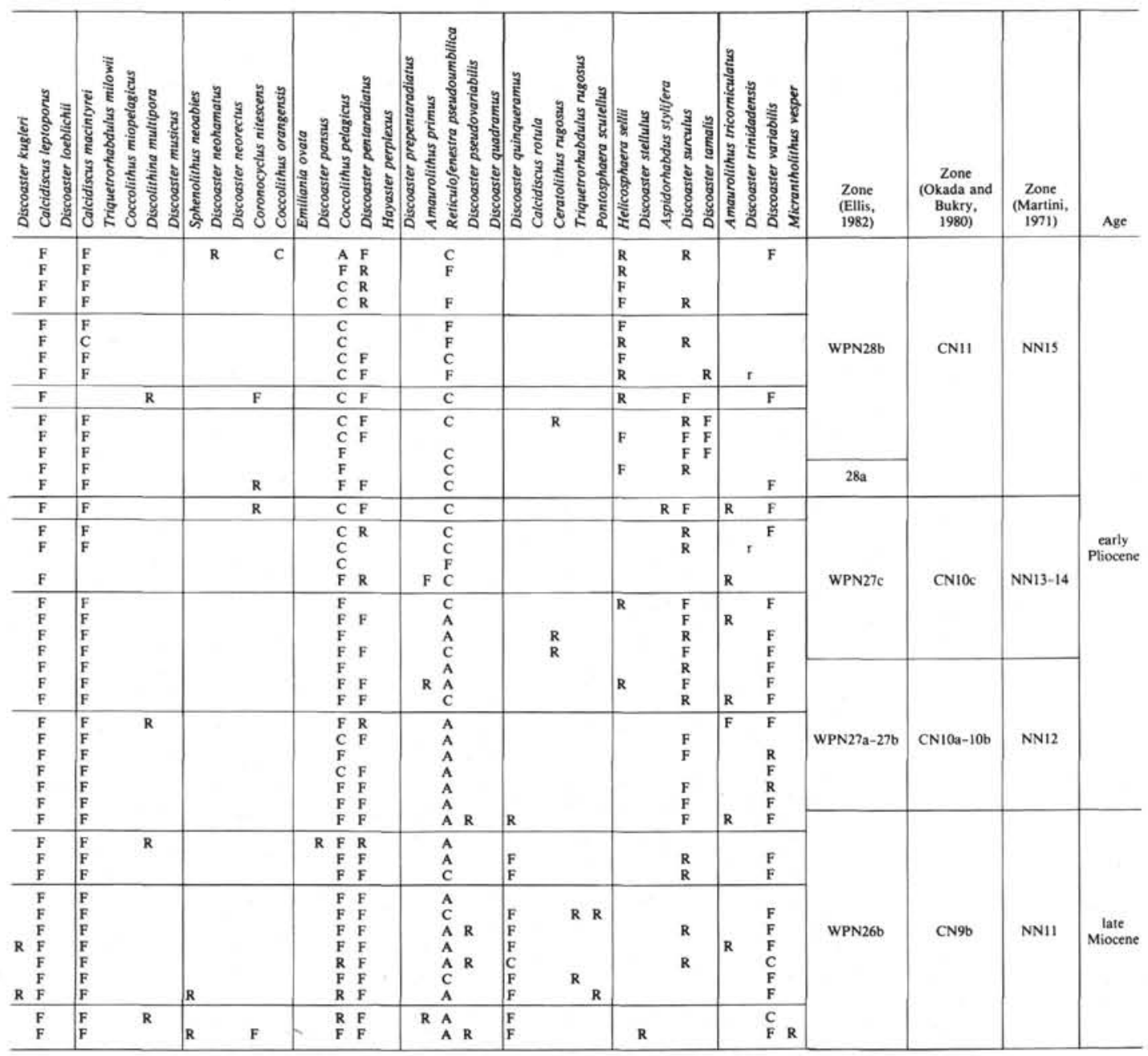




\section{J. C. STEINMETZ, H. STRADNER}

Table 2B. Late Miocene-middle Miocene. Hole 530A (Continued).

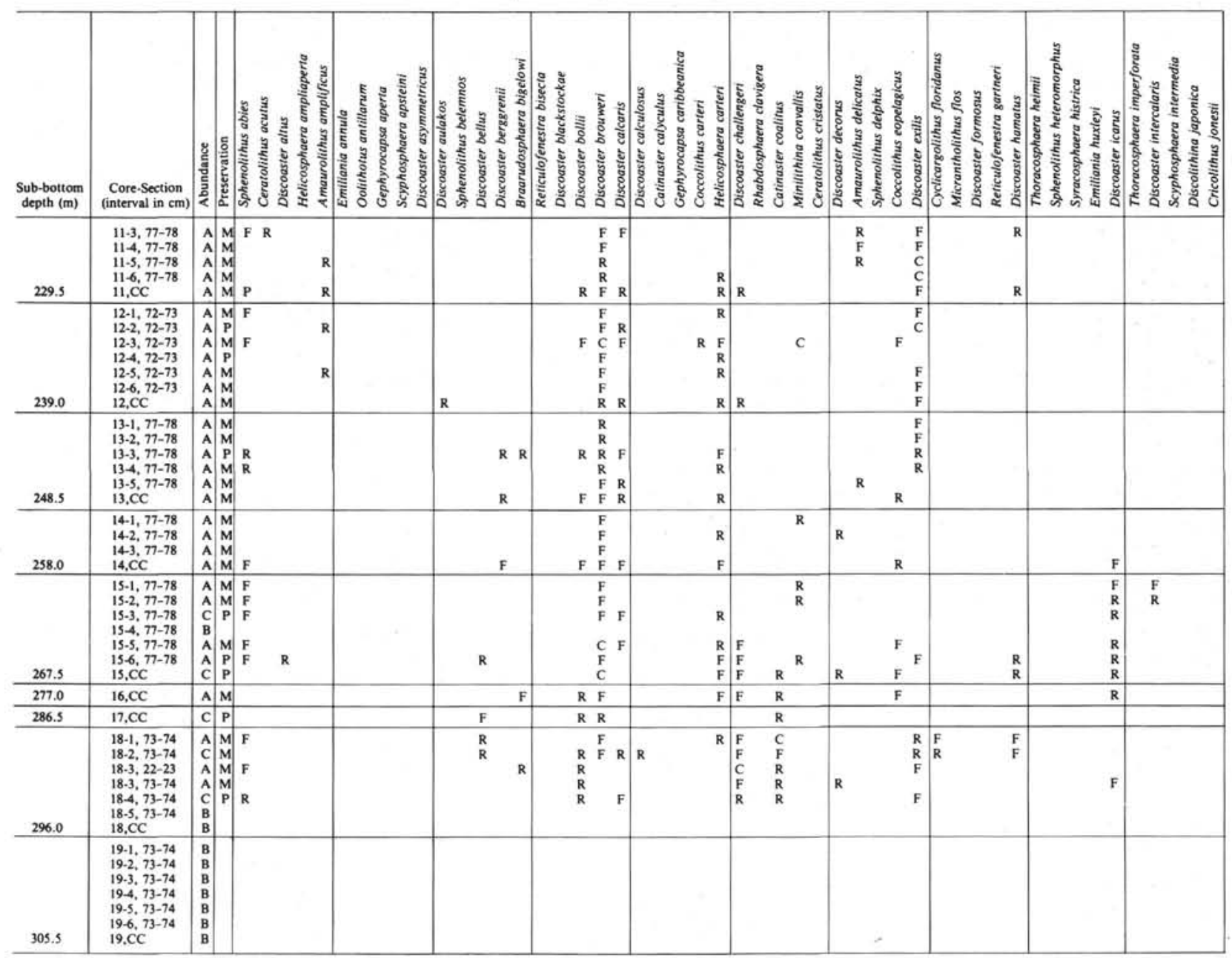


Table 2B. (Continued).

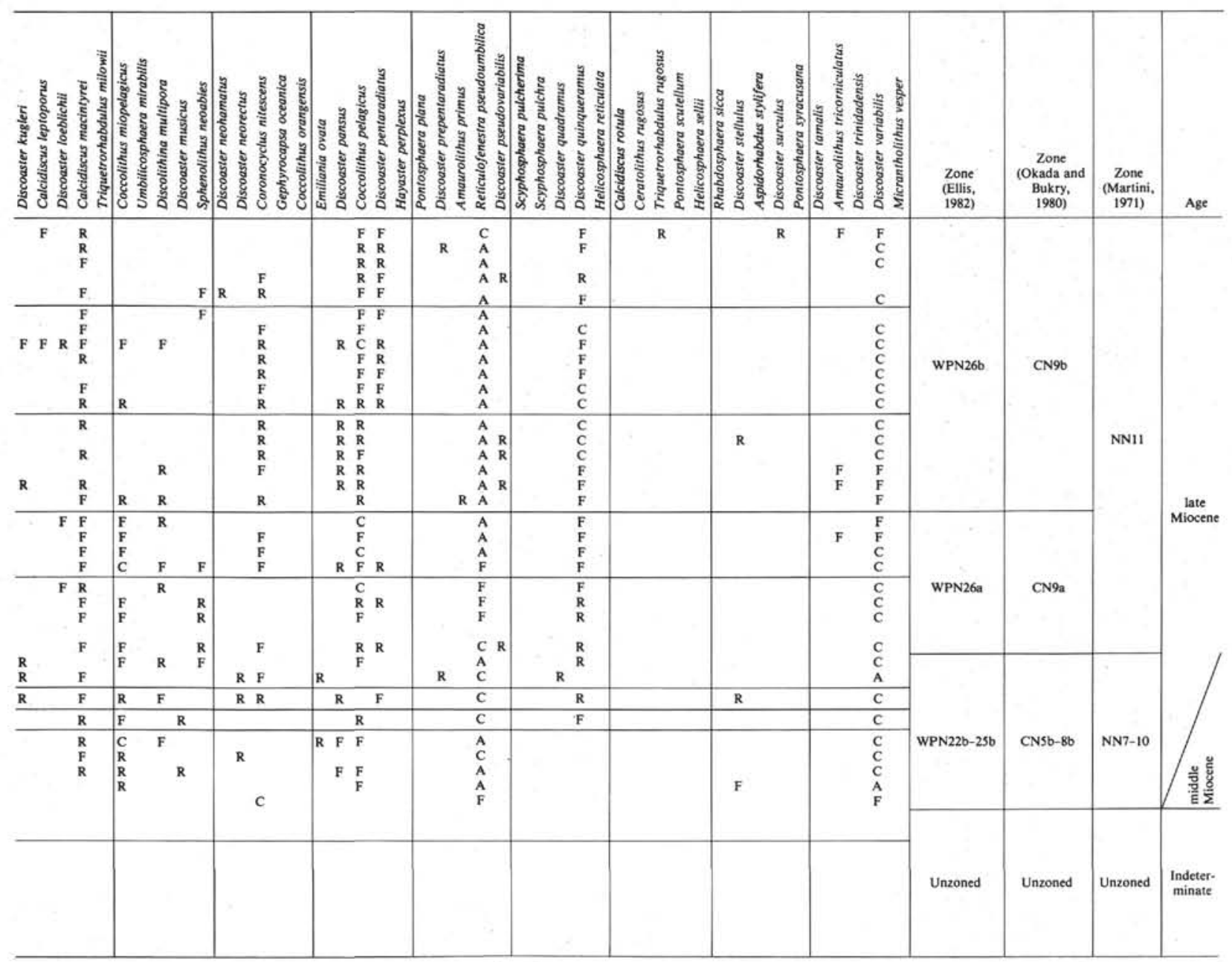


Table 2C. Middle Miocene. Hole 530A (continued).

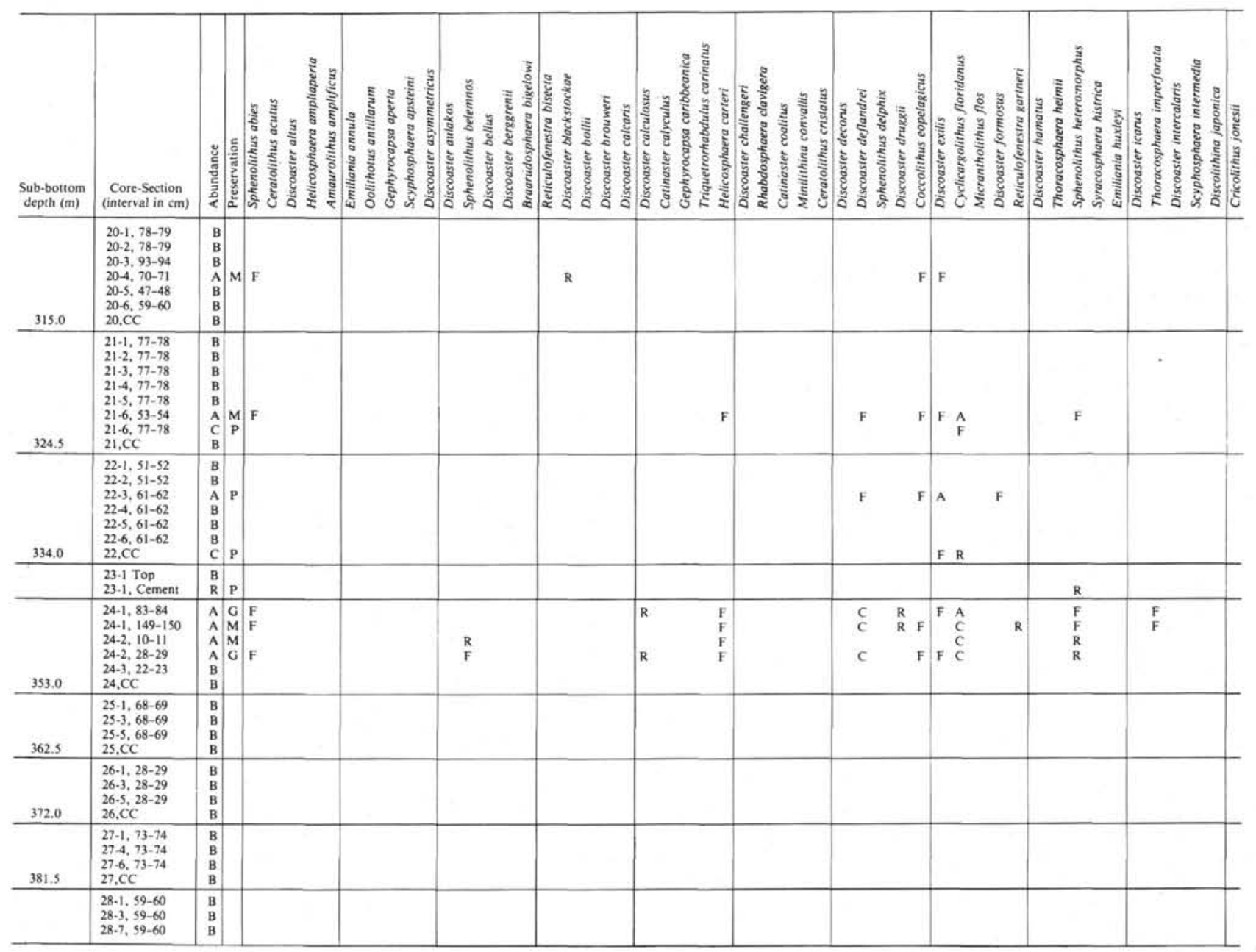


Table 2C. (Continued).

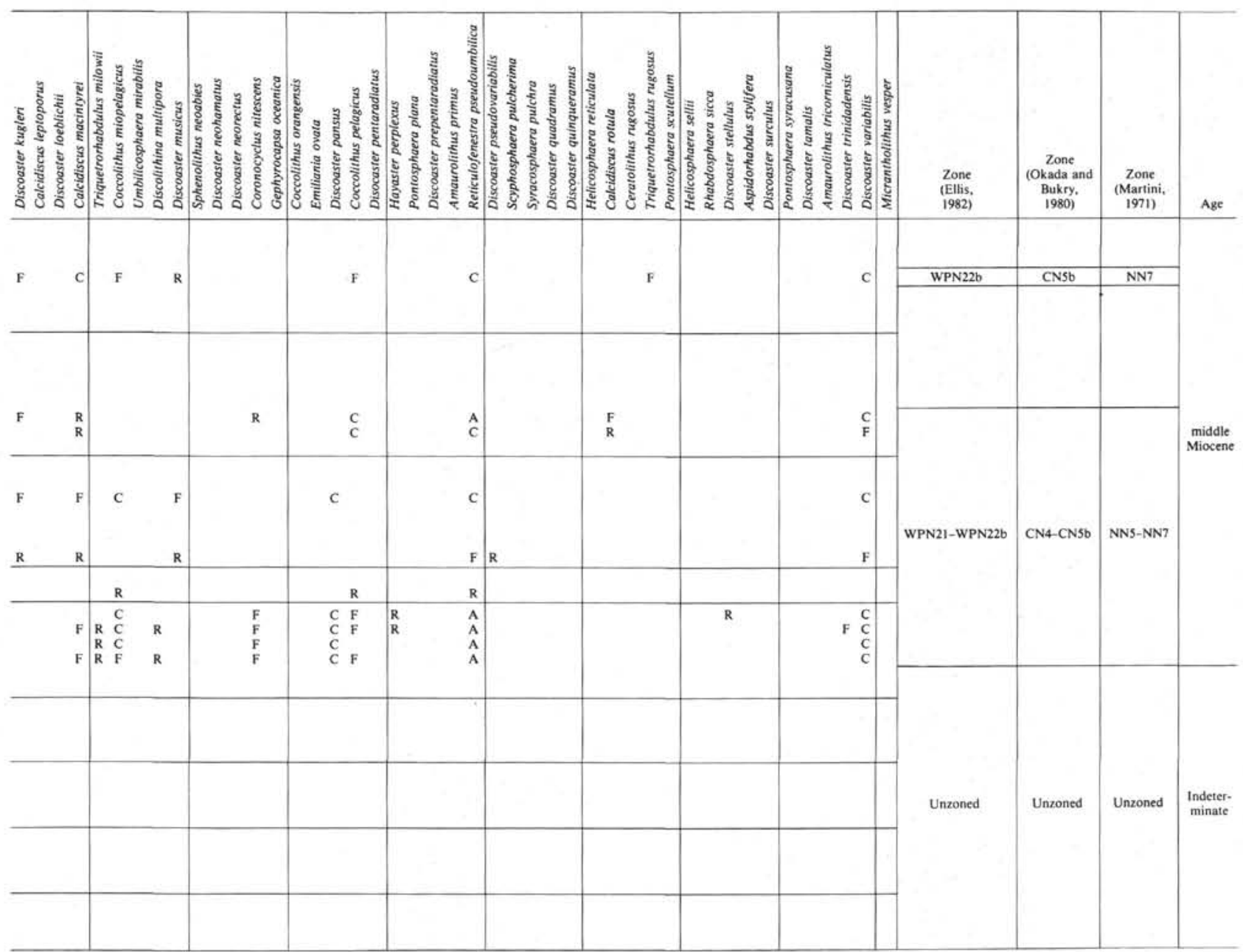


Table 2D. Late Oligocene-late Eocene. Hole 530A (continued).

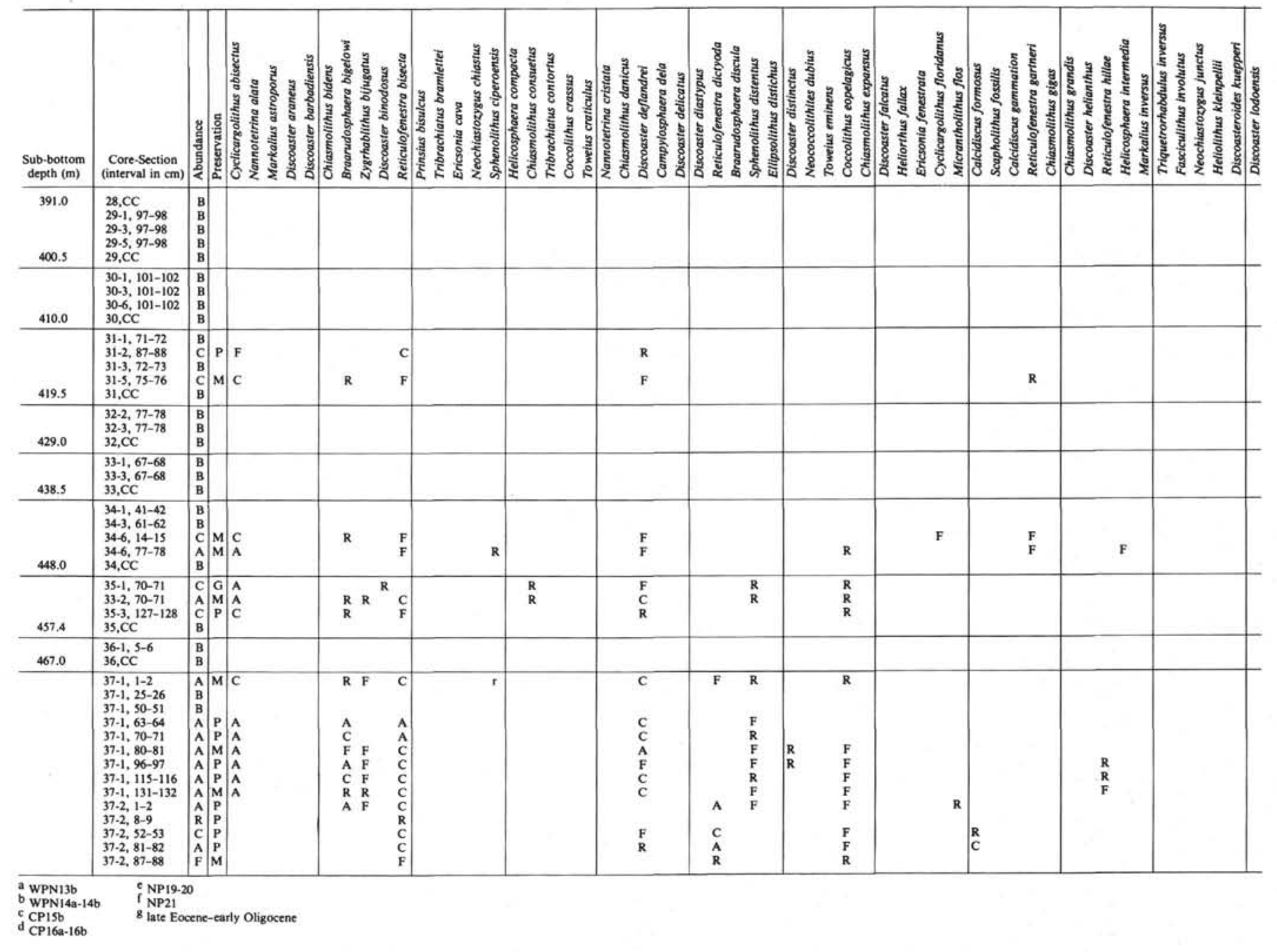


Table 2D. (Continued).

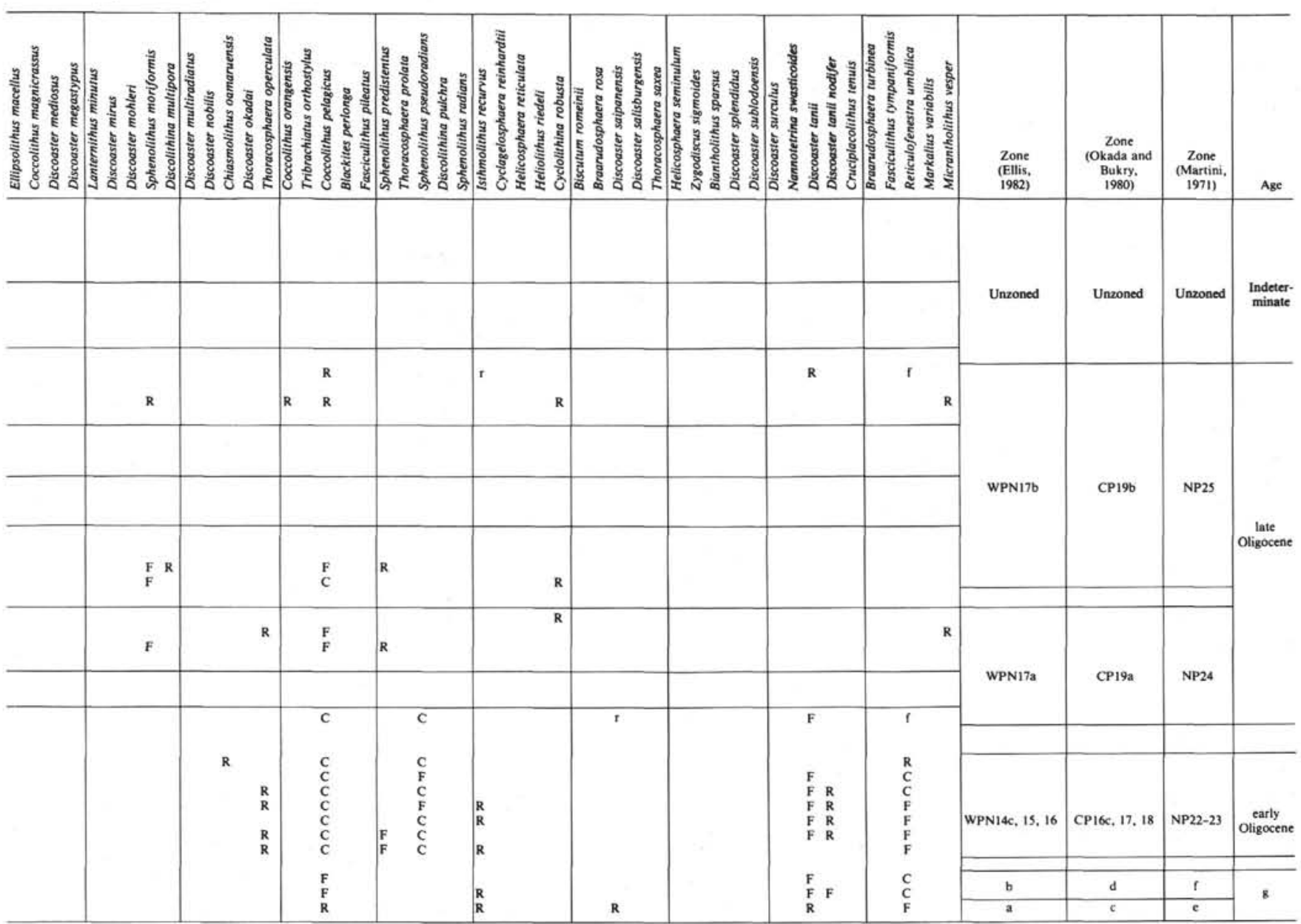


Table 2E. Middle Eocene-late Paleocene. Hole 530A (continued).

\begin{tabular}{|c|c|c|c|c|c|c|c|c|c|c|c|c|c|}
\hline $\begin{array}{l}\text { Subbottom } \\
\text { depth (m) }\end{array}$ & $\begin{array}{c}\text { Core-Section } \\
\text { (interval in } \mathrm{cm} \text { ) }\end{array}$ & & 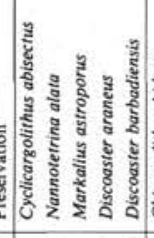 & 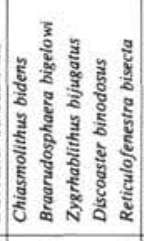 & 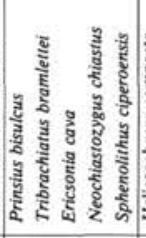 & 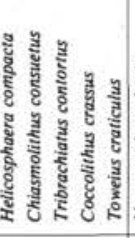 & 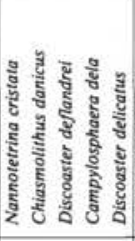 & 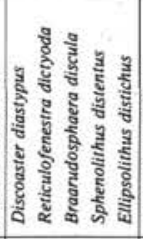 & 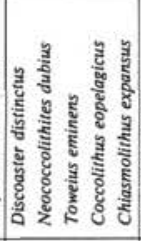 & 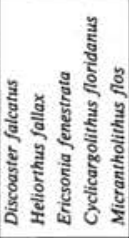 & 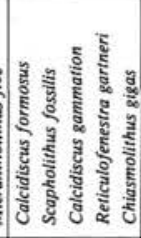 & 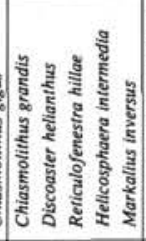 & 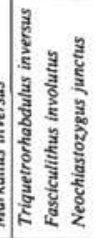 \\
\hline 476.5 & $\begin{array}{l}37-2,105-106 \\
37-2,148-149 \\
37-3,15-16 \\
37-3,25-30 \\
37-3,40-41 \\
37, c C \\
\end{array}$ & \begin{tabular}{|l|l|}
$\mathrm{C}$ & $\mathrm{M}$ \\
$\mathrm{B}$ & \\
$\mathrm{C}$ & $\mathrm{P}$ \\
$\mathrm{A}$ & $\mathrm{P}$ \\
$\mathrm{R}$ & $\mathrm{P}$ \\
$\mathrm{C}$ & $\mathrm{P}$ \\
\end{tabular} & $\begin{array}{ll}\mathbf{R} & \mathrm{F} \\
\mathbf{F} & \mathrm{F} \\
\mathrm{R} & \mathrm{C} \\
\mathbf{R} & \mathrm{C} \\
\end{array}$ & $\begin{array}{lll} & & C \\
& & \\
& F & \\
\end{array}$ & & $\begin{array}{l}\mathrm{R} \\
\mathrm{F} \\
\mathrm{F} \\
\end{array}$ & $R$ & $\begin{array}{l}\text { c } \\
\text { c }\end{array}$ & R & $\begin{array}{ll} & F \\
R & \\
R & \\
\end{array}$ & R & $\mid \begin{array}{l}C \\
\text { R }\end{array}$ & $\mathrm{F}$ \\
\hline 486.0 & $\begin{array}{l}38-1,39-40 \\
38-1,74-75 \\
38-1,86-87 \\
38-1,134-135 \\
38-2,1-2 \\
38-2,50-51 \\
38-2,105-106 \\
38-2,133-134 \\
38, C C\end{array}$ & \begin{tabular}{|l|l|}
$\mathrm{F}$ & $\mathrm{P}$ \\
$\mathrm{C}$ & $\mathrm{P}$ \\
$\mathrm{B}$ & \\
$\mathrm{B}$ & \\
$\mathrm{A}$ & $\mathrm{P}$ \\
$\mathrm{B}$ & \\
$\mathrm{B}$ & \\
$\mathrm{F}$ & $\mathrm{P}$ \\
$\mathrm{C}$ & $\mathrm{P}$ \\
\end{tabular} & P & $\begin{array}{r}\mathrm{R} C \mathrm{C} \\
\quad \mathrm{C}\end{array}$ & & & & F F & $\begin{array}{l}\mathrm{R} \\
\mathrm{F}\end{array}$ & & $\begin{array}{l}F \\
F\end{array}$ & $\mathbf{F}$ & \\
\hline 495.5 & $\begin{array}{l}39-1,8-9 \\
39-1,48-49 \\
39-1,85-86 \\
39-1,110-111 \\
39-2,5-6-6 \\
39-2,11-20 \\
39-2,42-43 \\
39-2,28-81 \\
39-2,120-121 \\
39, C C\end{array}$ & \begin{tabular}{|l|l}
$\mathrm{C}$ & $\mathrm{P}$ \\
$\mathrm{C}$ & $\mathrm{p}$ \\
$\mathrm{A}$ & $\mathrm{P}$ \\
$\mathrm{C}$ & $\mathrm{P}$ \\
$\mathrm{B}$ & $\mathrm{P}$ \\
$\mathrm{C}$ & $\mathrm{P}$ \\
$\mathrm{A}$ & $\mathrm{P}$ \\
$\mathrm{C}$ & $\mathrm{P}$ \\
$\mathrm{A}$ & $\mathrm{P}$ \\
$\mathrm{C}$ & $\mathrm{P}$
\end{tabular} & $\begin{array}{l}\mathrm{F} \\
\mathrm{F} \\
\mathrm{c} \\
\mathrm{c} \\
\mathrm{c} \\
\mathrm{c} \\
\mathrm{c} \\
\mathrm{c} \\
\mathrm{F}\end{array}$ & $\begin{array}{l}\mathrm{C} \\
\mathrm{F} \\
\mathrm{F}\end{array}$ & & $\begin{array}{l}F \\
F\end{array}$ & R & & $\mathbf{F}$ & & $\begin{array}{ll}R & R \\
& \\
F & \\
& \\
F & \end{array}$ & $\begin{array}{l}\mathrm{F} \\
\mathrm{R} \\
\mathrm{F}\end{array}$ & \\
\hline \multirow[t]{2}{*}{505.0} & $\begin{array}{l}40-1,10-11 \\
40-1,75-76 \\
40-1,140-141 \\
40-2,13-14 \\
40-2,80-81 \\
40-2,140-141 \\
40-3,8-9 \\
40-3,68-69 \\
40-3,122-123 \\
40-4,9-10 \\
40-4,72-73 \\
40-4,92-93 \\
40,0 \mathrm{C}\end{array}$ & \begin{tabular}{|l|l}
$\mathrm{B}$ & \\
$\mathrm{C}$ & $\mathrm{P}$ \\
$\mathrm{C}$ & $\mathrm{P}$ \\
$\mathrm{B}$ & \\
$\mathrm{A}$ & $\mathrm{P}$ \\
$\mathrm{A}$ & $\mathrm{P}$ \\
$\mathrm{A}$ & $\mathrm{P}$ \\
$\mathrm{A}$ & $\mathrm{P}$ \\
$\mathrm{A}$ & $\mathrm{P}$ \\
$\mathrm{A}$ & $\mathrm{P}$ \\
$\mathrm{A}$ & $\mathrm{P}$ \\
$\mathrm{A}$ & $\mathrm{P}$ \\
$\mathrm{A}$ & $\mathrm{P}$ \\
\end{tabular} & $\begin{array}{rl}\mathrm{c} \\
\mathrm{A} \\
\mathrm{F} & \mathrm{c} \\
\mathrm{c} & \end{array}$ & 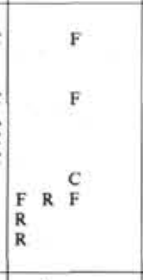 & $\begin{array}{ll}A & R \\
A & \\
A \\
A \\
A & R \\
\end{array}$ & $\begin{array}{l}\text { F } \\
\text { F } \\
\text { R }\end{array}$ & & $\begin{array}{l}\mathrm{C} \\
\mathrm{F} \\
\mathrm{F}\end{array}$ & $\mathbf{F}$ & $\begin{array}{l}\mathbf{F} \\
\mathbf{R} \\
\mathbf{R}\end{array}$ & $c$ & & $\begin{array}{l}\mathrm{A} \\
\mathrm{A} \\
\mathrm{A} \\
\mathrm{A}\end{array}$ \\
\hline & $\begin{array}{l}41-1,33-34 \\
41-1,75-76 \\
41-1,9-91 \\
41-1,141-142 \\
41-2,10-11 \\
41 \cdot 2,70-71 \\
41 \cdot 2,110-111\end{array}$ & \begin{tabular}{|l|l}
$\mathrm{A}$ & $\mathrm{P}$ \\
$\mathrm{A}$ & $\mathrm{P}$ \\
$\mathrm{B}$ & \\
$\mathrm{C}$ & $\mathrm{P}$ \\
$\mathrm{A}$ & $\mathrm{P}$ \\
$\mathrm{A}$ & $\mathrm{P}$ \\
$\mathrm{A}$ & $\mathrm{P}$
\end{tabular} & & $R$ & $\begin{array}{l}\text { A } \\
\text { A R } \\
\text { R } \\
\text { C } \\
C \\
\text { C }\end{array}$ & $\begin{array}{l}\mathrm{C} \\
\mathrm{F} \\
\mathrm{F} \\
\mathrm{F}\end{array}$ & & & $\begin{array}{l}\mathrm{R} \\
\mathrm{C} \\
\mathrm{F} \\
\mathrm{C}\end{array}$ & & & & $\begin{array}{l}\text { A } \\
\text { C } \\
\text { R } \\
\text { C } \\
\text { C } \\
\text { C }\end{array}$ \\
\hline
\end{tabular}


Table 2E. (Continued).

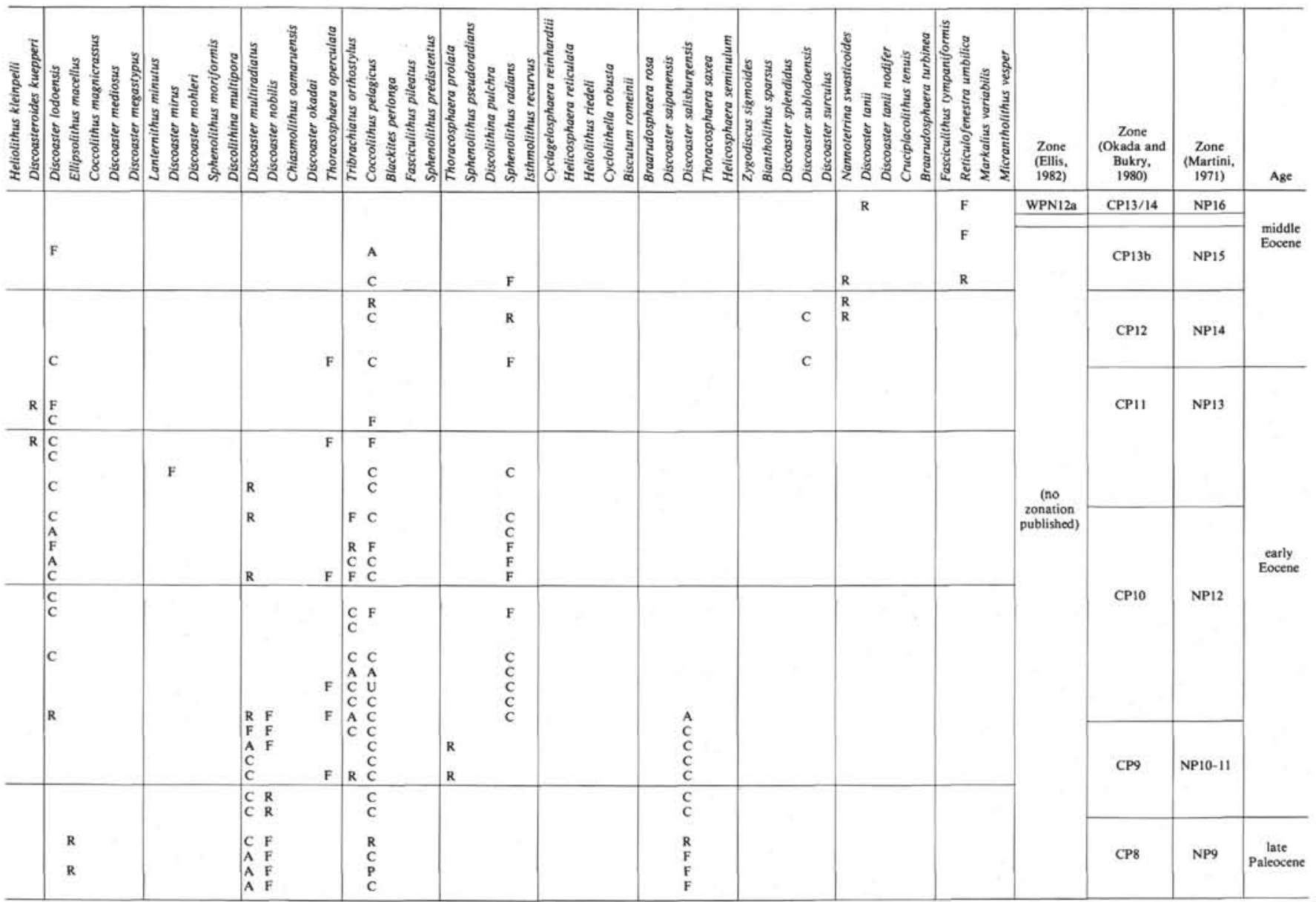


Table 2F. Late Paleocene-early Paleocene. Hole 530A (Continued).

\begin{tabular}{|c|c|c|c|c|c|c|c|c|c|c|c|c|c|c|c|}
\hline $\begin{array}{l}\text { Sub-bottom } \\
\text { depth (m) }\end{array}$ & $\begin{array}{c}\text { Core-Section } \\
\text { (interval in cm) }\end{array}$ & 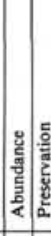 & & 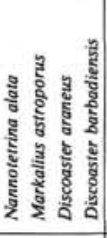 & 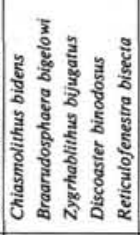 & 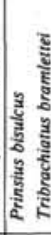 & 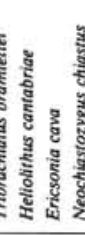 & 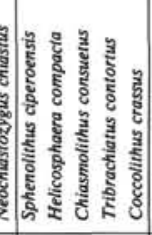 & 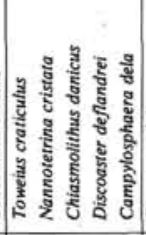 & 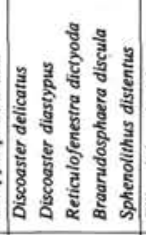 & 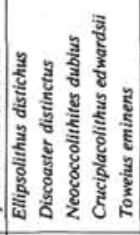 & 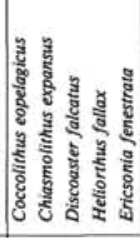 & 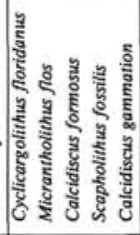 & 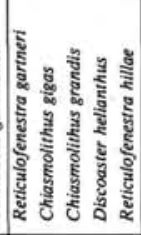 & 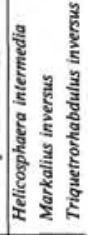 \\
\hline 514.5 & $\begin{array}{l}41-3,8-9 \\
41-3,80-81 \\
41-3,140-141 \\
41, C C\end{array}$ & \begin{tabular}{|l|l}
$A$ & : \\
$A$ & : \\
$A$ & $N$ \\
$A$ & $N$ \\
\end{tabular} & & & $\begin{array}{l}F \\
R \\
R \quad R \\
R\end{array}$ & $\begin{array}{l}\mathrm{C} \\
\mathrm{C} \\
\mathrm{C} \\
\mathrm{F} \\
\mathrm{F}\end{array}$ & R & & & & $\begin{array}{l}\mathrm{c} \\
\mathrm{c} \\
\mathrm{C} \\
\mathrm{C} \\
\end{array}$ & R & & & \\
\hline 524.0 & $\begin{array}{l}42-1,17-18 \\
42-1,61-62 \\
42-1,140-141 \\
42-2,20-21 \\
42-2,63-64 \\
42-2,140-141 \\
42-3,10-11 \\
42, \mathrm{CC} \\
\end{array}$ & \begin{tabular}{|l|l|}
$A$ & $F$ \\
$A$ & P \\
A & P \\
A & P \\
B & P \\
A & P \\
A & P \\
\end{tabular} & & & $\begin{array}{l}\mathrm{F} \\
\mathrm{F} \\
\mathrm{F} \\
\mathrm{R} \\
\mathrm{R} \\
\mathrm{R} \\
\mathrm{R} \\
\end{array}$ & $\begin{array}{l}\mathrm{F} \\
\mathrm{F} \\
\mathrm{C} \\
\mathrm{A} \\
\mathrm{A} \\
\mathrm{A} \\
\mathrm{A} \\
\end{array}$ & & & $\begin{array}{l}\mathrm{F} \\
\mathrm{C} \\
\mathrm{C} \\
\mathrm{C} \\
\end{array}$ & R & 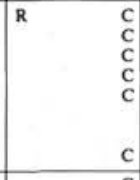 & $\begin{array}{l}R \\
R \\
R \\
R \\
R \\
F \\
F \\
F\end{array}$ & & $\begin{array}{l}\mathrm{R} \\
\mathrm{R} \\
\end{array}$ & - \\
\hline 533.5 & $\begin{array}{l}43-1,14-15 \\
43-1,57-58 \\
43-1,110-111 \\
43-2,20-21 \\
43-2,76-77 \\
43-2,122-123 \\
43, C C\end{array}$ & \begin{tabular}{|l|l}
$\mathrm{A}$ & $\mathrm{M}$ \\
$\mathrm{C}$ & $\mathrm{P}$ \\
$\mathrm{A}$ & $\mathrm{P}$ \\
$\mathrm{A}$ & $\mathrm{M}$ \\
$\mathrm{A}$ & $\mathrm{N}$ \\
$\mathrm{A}$ & $\mathrm{P}$ \\
$\mathrm{C}$ & $\mathrm{P}$ \\
\end{tabular} & & & 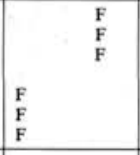 & $\begin{array}{ll}\mathrm{A} \\
\mathrm{A} \\
\mathrm{A} \\
\mathrm{C} \\
\mathrm{A} \\
\mathrm{A} \\
\mathrm{C} \\
\end{array}$ & R & $\begin{array}{l}\mathrm{F} \\
\mathrm{R} \\
\mathrm{R} \\
\mathrm{R} \\
\end{array}$ & $\begin{array}{l}\mathrm{C} \\
\mathrm{c} \\
\mathrm{c} \\
\mathrm{C} \\
\mathrm{F} \\
\mathrm{F} \\
\mathrm{F}\end{array}$ & & $\begin{array}{l}\text { C } \\
\text { c } \\
\text { C } \\
\text { C } \\
\text { F }\end{array}$ & & & $\begin{array}{l}\mathrm{F} \\
\mathrm{F} \\
\mathrm{F} \\
\mathrm{F}\end{array}$ & \\
\hline 543.0 & $\begin{array}{l}44-1,19-20 \\
44-1,69-70 \\
44-1,113-114 \\
44-1,129-130 \\
44-2,15-16 \\
44-2,75-76 \\
44-2,115-116 \\
44, C C\end{array}$ & \begin{tabular}{|l|l|}
$A$ & $P$ \\
$A$ & $P$ \\
$A$ & $M$ \\
$B$ & \\
$B$ & \\
$R$ & $P$ \\
$R$ & $P$ \\
$C$ & $P$ \\
\end{tabular} & & & & $\begin{array}{l}\mathrm{A} \\
\mathrm{A} \\
\mathrm{C} \\
\mathrm{R} \\
\mathrm{C}\end{array}$ & ${ }_{R}^{R}$ & $\mathrm{R}$ & $\begin{array}{l}\mathrm{R} \\
\mathrm{F}\end{array}$ & & $\begin{array}{l}\mathrm{F} \\
\mathrm{F}\end{array}$ & & & $=$ & \\
\hline 552.5 & $\begin{array}{l}\begin{array}{l}45-1,43-44 \\
45-1,57-58 \\
45, C C\end{array} \\
4.6 C\end{array}$ & \begin{tabular}{|l|l|l|}
$B$ & \\
$R$ & $P$ \\
$R$ & $P$ \\
\end{tabular} & & & & & $R$ & & & & & & & & \\
\hline 562.0 & $\begin{array}{l}\begin{array}{l}46-1,34-35 \\
46-1,50-51 \\
46, C C\end{array} \\
46,\end{array}$ & \begin{tabular}{l|l}
$R$ & $P$ \\
$A$ & $P$ \\
$C$ & $P$ \\
\end{tabular} & & & & c & $\begin{array}{l}\mathrm{c} \\
\mathrm{C} \\
\end{array}$ & & $\begin{array}{l}\mathrm{R} \\
\mathrm{F}\end{array}$ & & $\begin{array}{l}R \\
R \\
\end{array}$ & & & & \\
\hline 571.5 & $\begin{array}{l}47-1,18-19 \\
47-1,70-71 \\
47-1,143-144 \\
47-2,25-26 \\
47-2,75-76 \\
47, \mathrm{CC}\end{array}$ & \begin{tabular}{|l|l|}
$\mathrm{A}$ & $\mathrm{P}$ \\
$\mathrm{A}$ & $\mathrm{M}$ \\
$\mathrm{A}$ & $\mathrm{P}$ \\
$\mathrm{A}$ & $\mathrm{P}$ \\
$\mathrm{C}$ & $\mathrm{P}$ \\
$\mathrm{F}$ & $\mathrm{P}$ \\
\end{tabular} & & $R$ & & & $\begin{array}{l}\mathrm{F} \text { A } \\
\hat{A} \\
\mathrm{~A} \\
\mathrm{~A} \\
\mathrm{~F}\end{array}$ & $\mathbf{R}$ & \begin{tabular}{|ll}
$C$ & \\
$C$ & $R$ \\
& $R$
\end{tabular} & & $\begin{array}{l}\mathrm{R} \\
\mathrm{R} \\
\mathrm{R} \\
\mathrm{R} \\
\mathrm{R} \\
\end{array}$ & & & & \\
\hline 581.0 & $\begin{array}{l}48-1,40-41 \\
48-1,79-80 \\
48-1,127-128 \\
48-2,1-2 \\
48-2,43-44 \\
48, C C\end{array}$ & \begin{tabular}{|l|l|l}
$A$ & $P$ \\
$C$ & $P$ \\
$A$ & $P$ \\
$A$ & $P$ \\
$A$ & $P$ \\
$A$ & $P$ \\
\end{tabular} & $\begin{array}{l}\mathrm{P} \\
\mathrm{P} \\
\mathrm{P} \\
\mathrm{P} \\
\mathrm{P} \\
\mathrm{P}\end{array}$ & & & & $\begin{array}{l}\mathrm{C} \\
\mathrm{F} \\
\mathrm{A} \\
\mathrm{A} \\
\mathrm{A} \\
\mathrm{A}\end{array}$ & & & & $\begin{array}{l}\mathrm{F} \\
\mathrm{R} \\
\mathrm{F} \\
\mathrm{F} \\
\mathrm{F} \\
\mathrm{F}\end{array}$ & & & & $\begin{array}{l}\mathrm{R} \\
\mathrm{F} \\
\mathrm{F}\end{array}$ \\
\hline
\end{tabular}


Table 2F. (Continued).

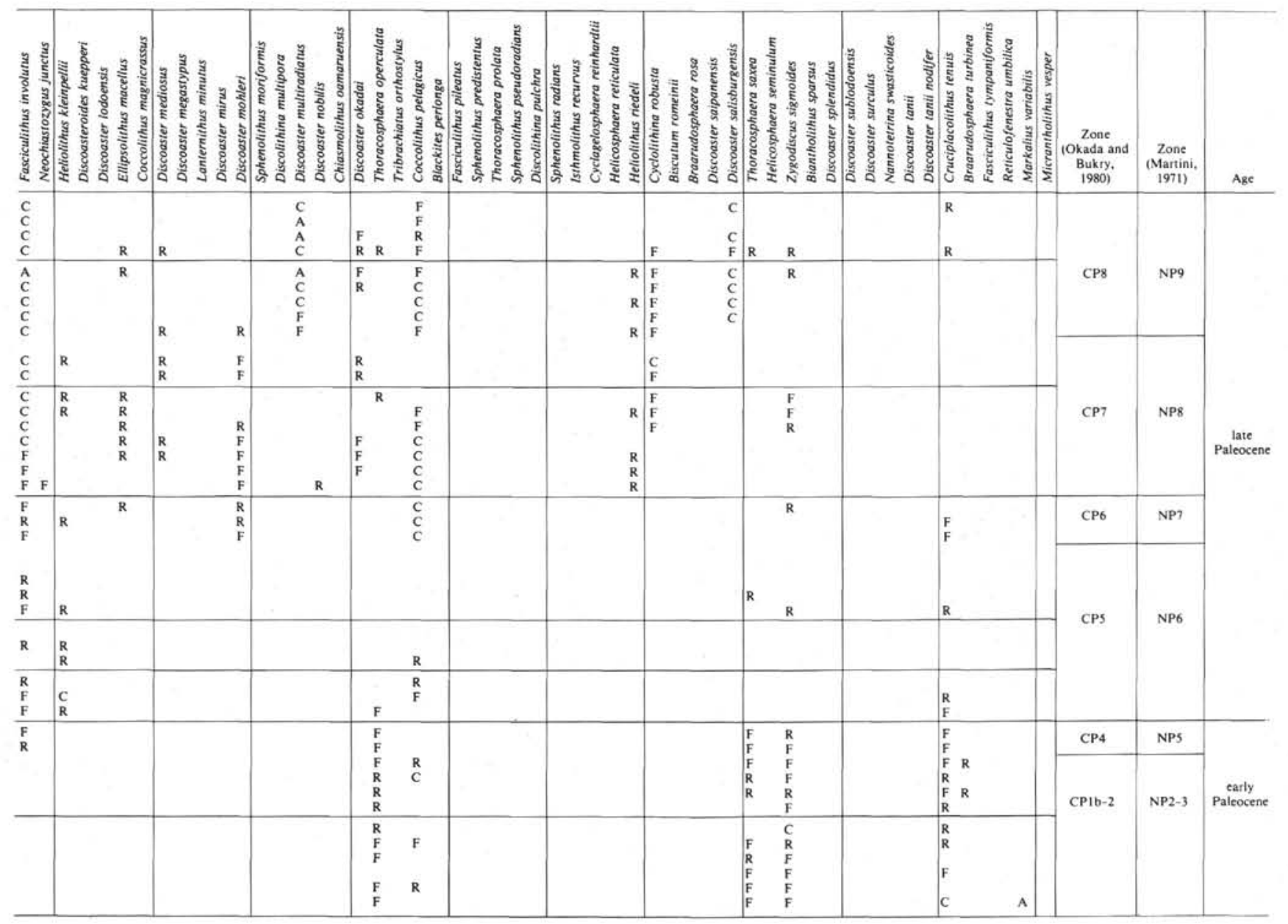




\section{J. C. STEINMETZ, H. STRADNER}

2G. Early Paleocene. Hole 530A (Continued).

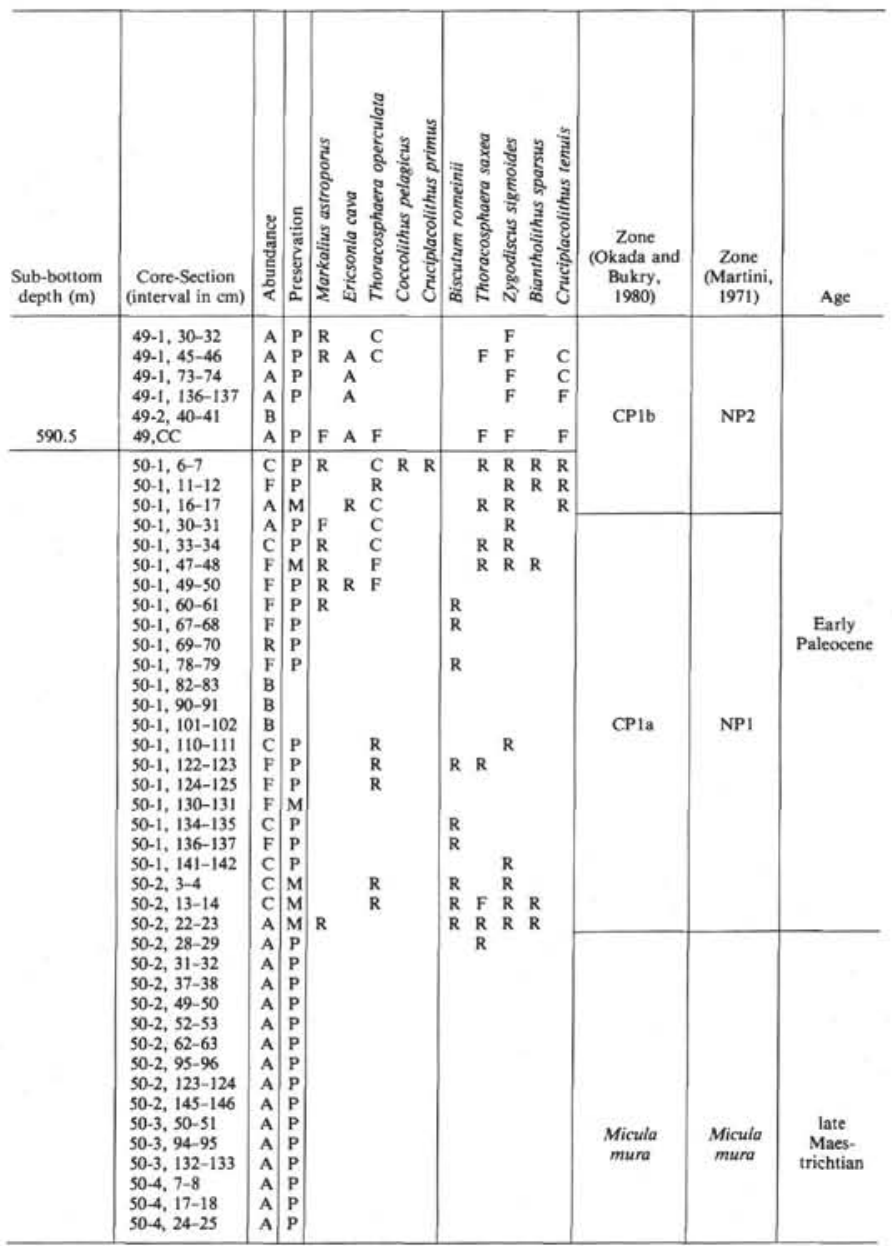

Note: The following species were not found: Cyclicargolithus abisectus, Biantholithus astralis, Discoaster arane. us, Discoaster barbadiensis, Chiasmolithus bidens, Braarudasphaera bigelowi, Zygrhablithus bijugatus, Discoaster binodosus, Reticulofenestra bisecta, Prinsius biswlcus, Tribrachiatus bramientei, Neochiastozygus chiastus, Sphenolithus ciperoensis, Helicasphaera compacta, Chiasmolithus consuens, Tribrachiatus contortus,

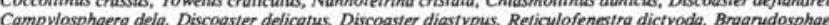
ra discula, Sphenolithus distentus, Ellipsolithus distichus, Discoaster distinctus, Neococoolithites dubius, Toweius eminens, Coccolithus eopelagicus, Chiasmolithus expansus, Discoaster falcatus, Heliorthus fallax, Ericsonia fenestrata, Cydlicargolithus foridanus, Micrantholithus fos, Catcidiscus formosus, Scapholithus fossilis, Cyclococcolithus gammation, Calcidiscus gammation, Chiasmolithus gigas, Chiasmolithus grandis, Discoaster helianthus, Reticulofenestra hillae, Helicosphaera intermedia, Markalius inversus, Triquetrorhab dulus inversus, Fasciculithus involutus, Neachiastozygus junctus, Heliolithus kleinpellii, Discoaster kuepperi, Discoaster lodoensis, Ellipsolithus macellas, Coccolithus magnicrassus, Discoaster mediasus, Discoaster megastypus, Lanternithus minutus, Discoaster mirus, Discoaster mohler, Sphenolithus moriformis, Disco. lithina multipara, Discoaster multiradiatus, Discoaster nobiils, Chiasmolithus oamaruensis, Discoaster oka-

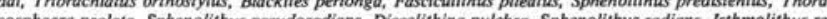

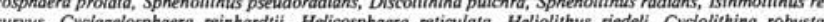
Bragrudosphaera rosa, Discoaster saipanensis, Discogster salishurgensis, Helicasphaera seminulum, Discoas. ter splendidus, Discoaster sublodoensis, Discoaster surculus, Nannotetrina swersticoidea, Discooster tanii, Discoaster tanii nodifer, Bragrudasphaera turbinea, Fasciculithus tympaniformis, Reticulofenestra umbilico, Markalius variabilis, Micrantholithus vesper 
Table 2H. Tertiary/Cretaceous boundary. Hole 530A (Continued).

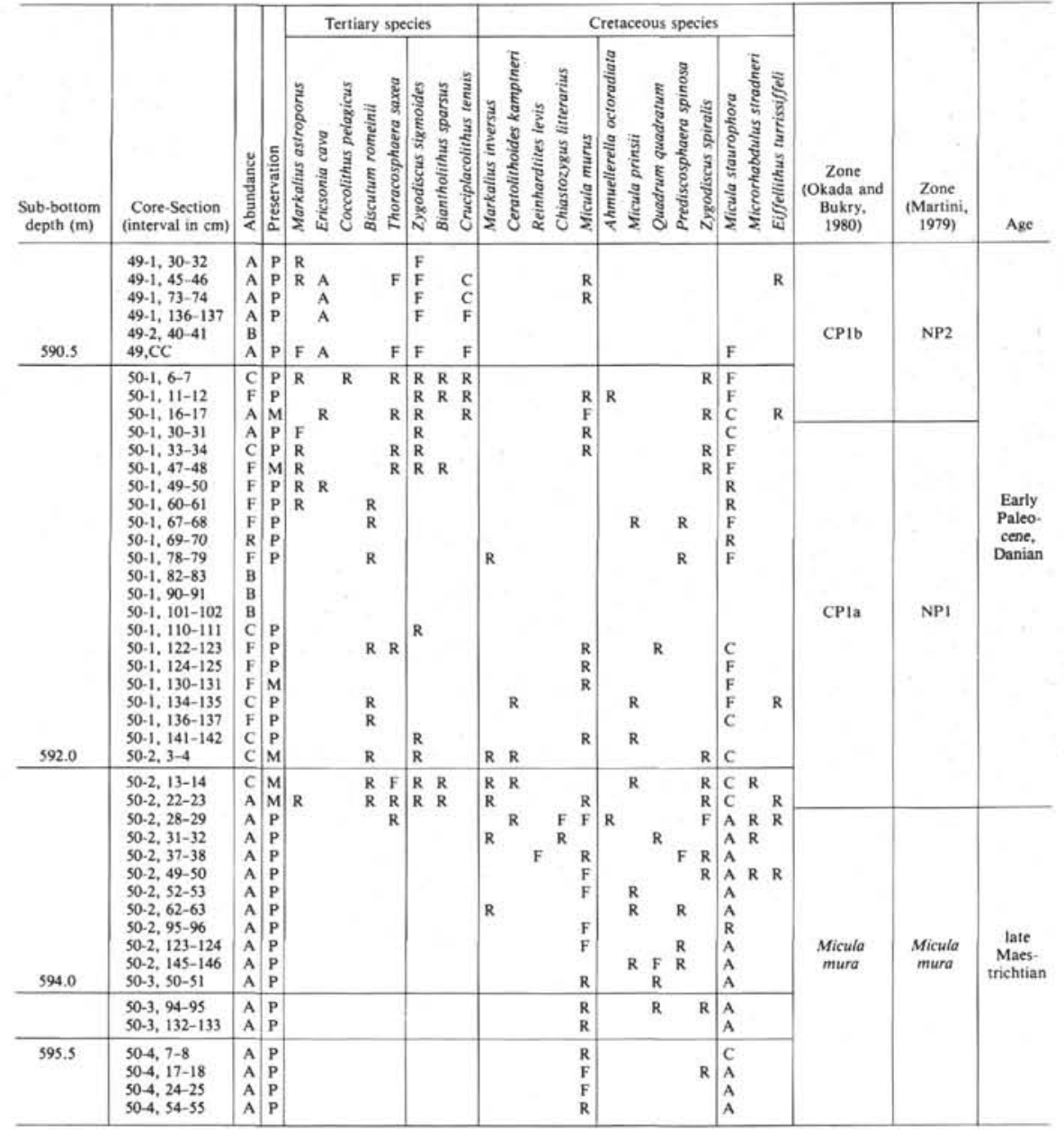


Table 3. Distribution of Holocene-late Miocene calcareous nannofossils, Hole 530B.

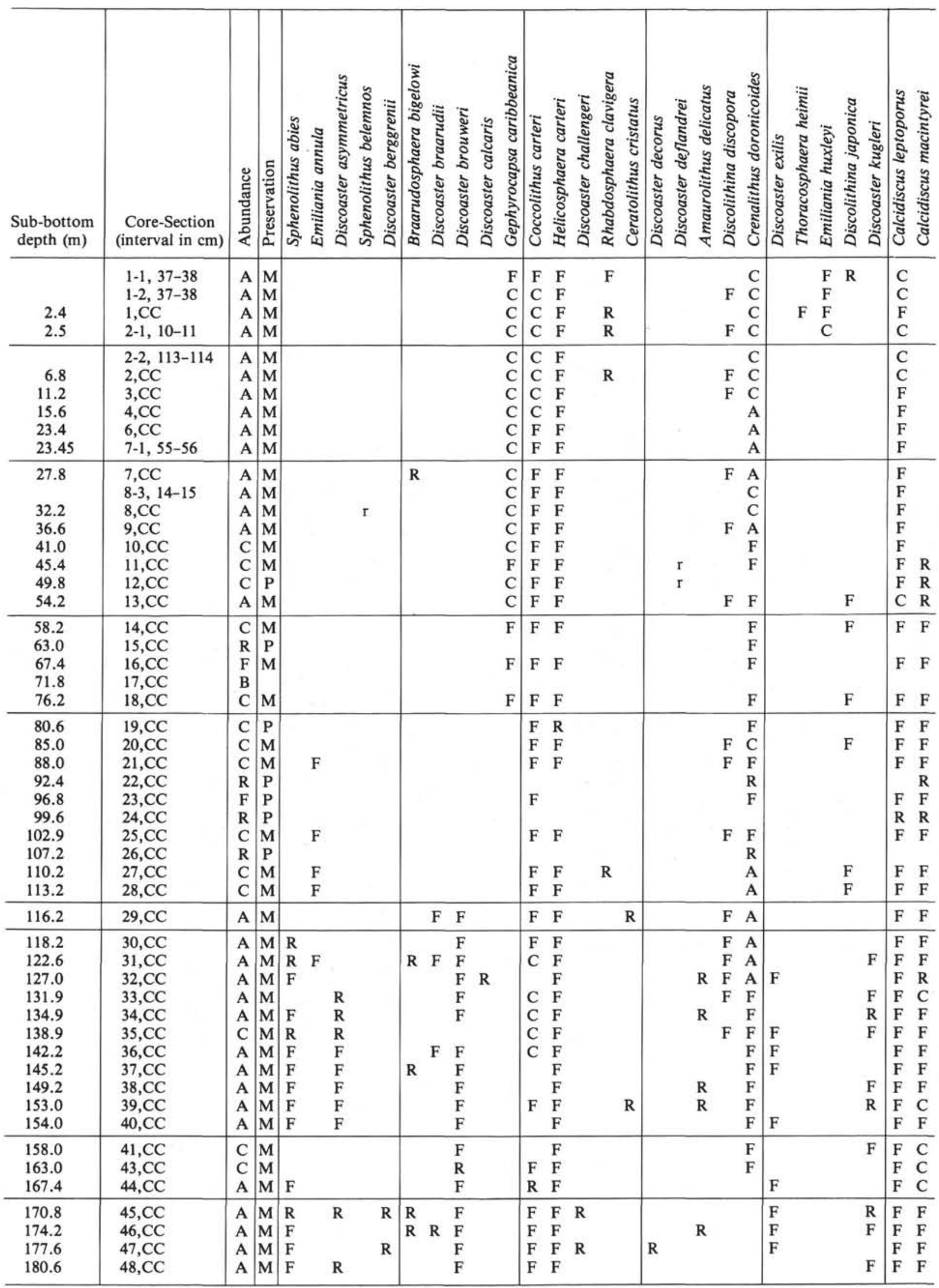

Note: Abundance: $\mathrm{A}=$ abundant $\mathrm{C}=$ common; $\mathrm{F}=$ few; $\mathrm{R}=$ rare; $\mathrm{B}=$ barren; lower case letter $=$ reworked older fossils. Preservation: $\mathrm{G}=$ good; $\mathrm{M}=$ moderate; $\mathrm{P}=$ poor. 
Table 3. (Continued).

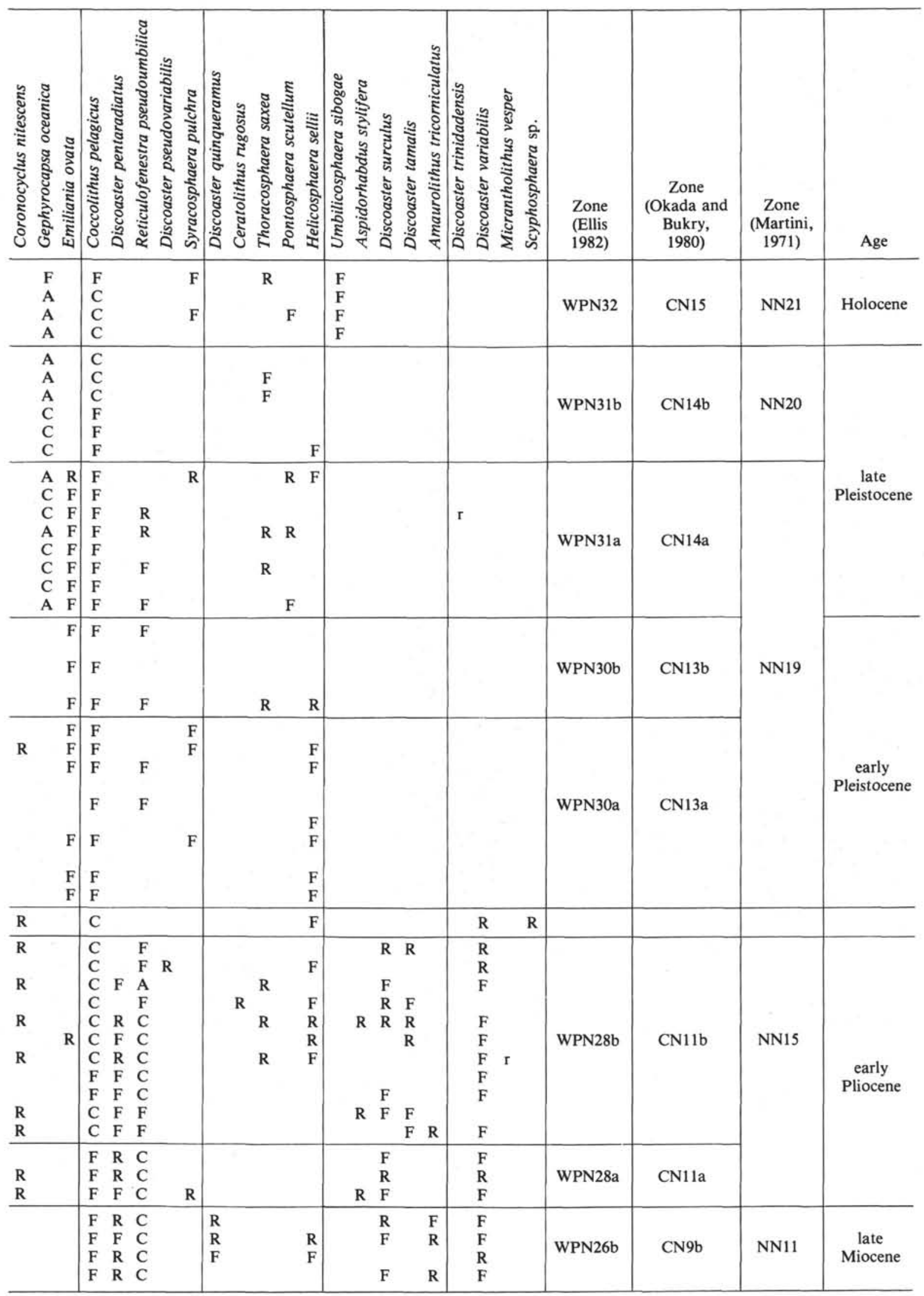


Table 4. Distribution of Holocene-late Miocene calcareous nannofossils, Holes 532, 532B.

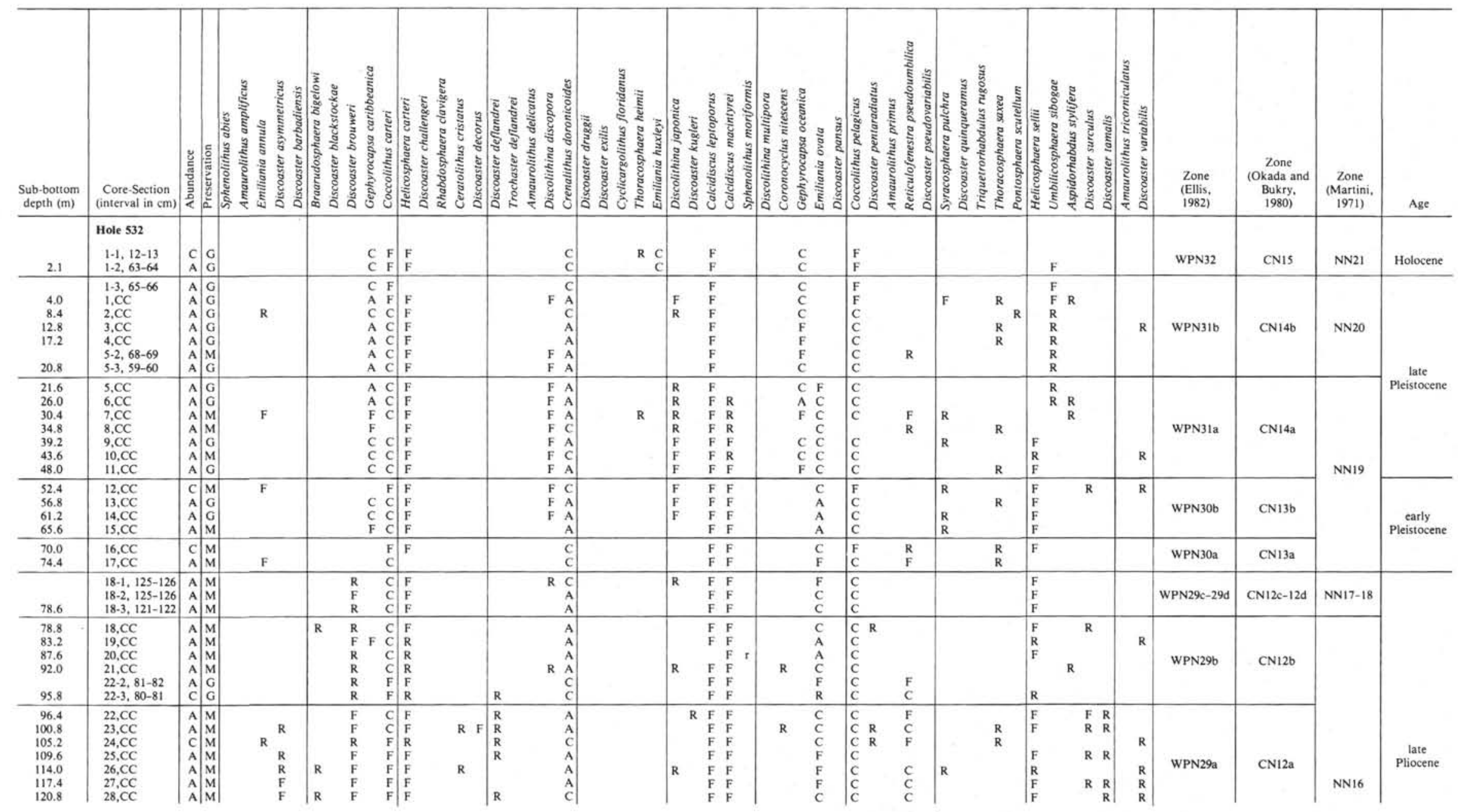


Table 4. (Continued).

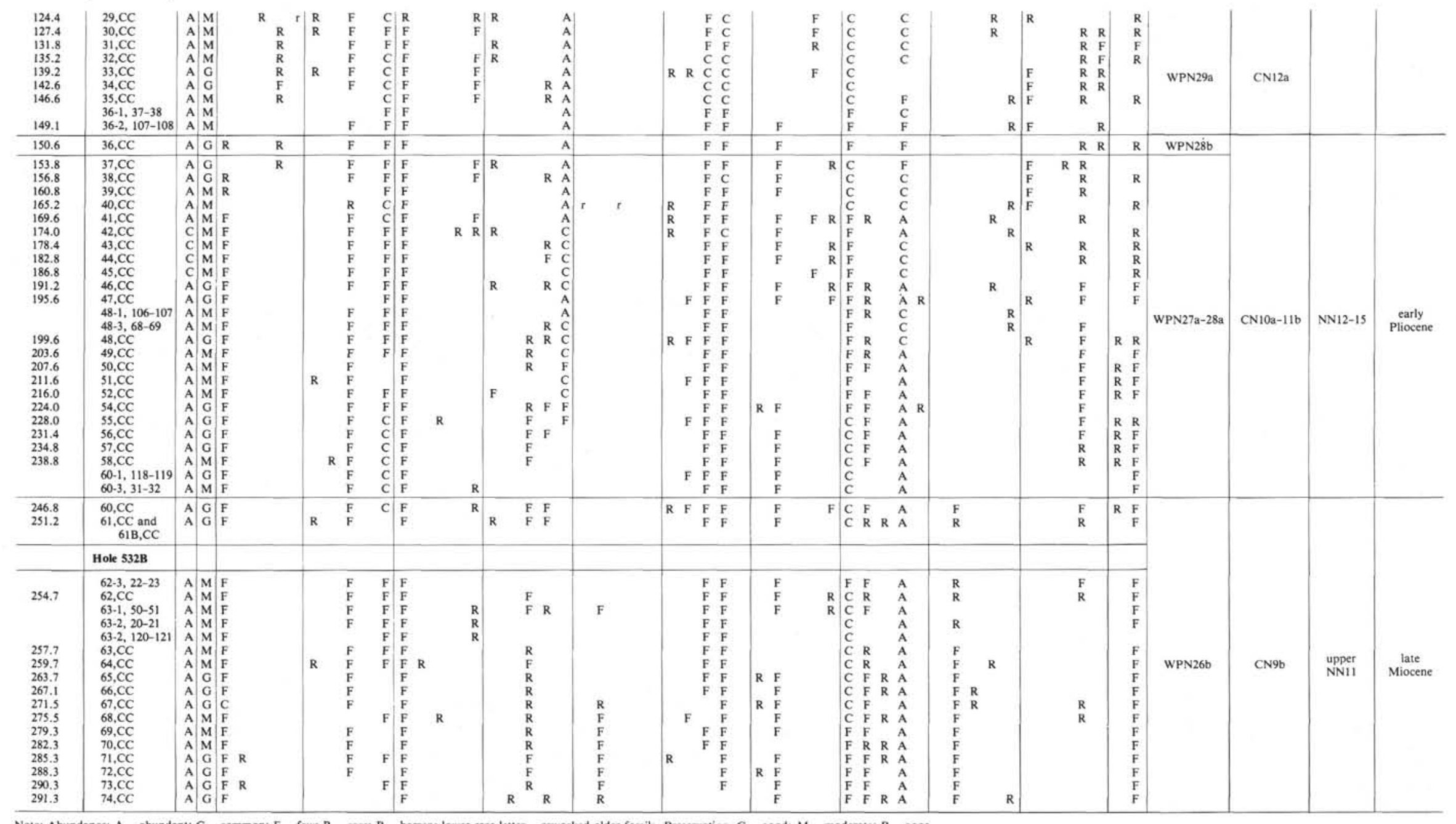


Table 5. Frequency estimate designations.

\begin{tabular}{lclc}
\hline Abundance of specimens & Logarithm & Designation & $\begin{array}{c}\text { Letter } \\
\text { designation }\end{array}$ \\
\hline $\begin{array}{c}\text { 10-100 specimens per } \\
\text { field of view }\end{array}$ & +1 & Abundant & $\mathrm{A}$ \\
$\begin{array}{c}1-10 \text { specimens per } \\
\text { field of view }\end{array}$ & 0 & Common & $\mathrm{C}$ \\
$\begin{array}{c}\text { Single specimen in 1-10 } \\
\text { fields of view }\end{array}$ & -1 & Few & $\mathrm{F}$ \\
$\begin{array}{c}\text { Single specimen in 10-100 } \\
\text { fields of view }\end{array}$ & -2 & Rare & $\mathrm{R}$ \\
\hline
\end{tabular}


Table 6. Geologic age and nannoplankton zone assignment of cores.

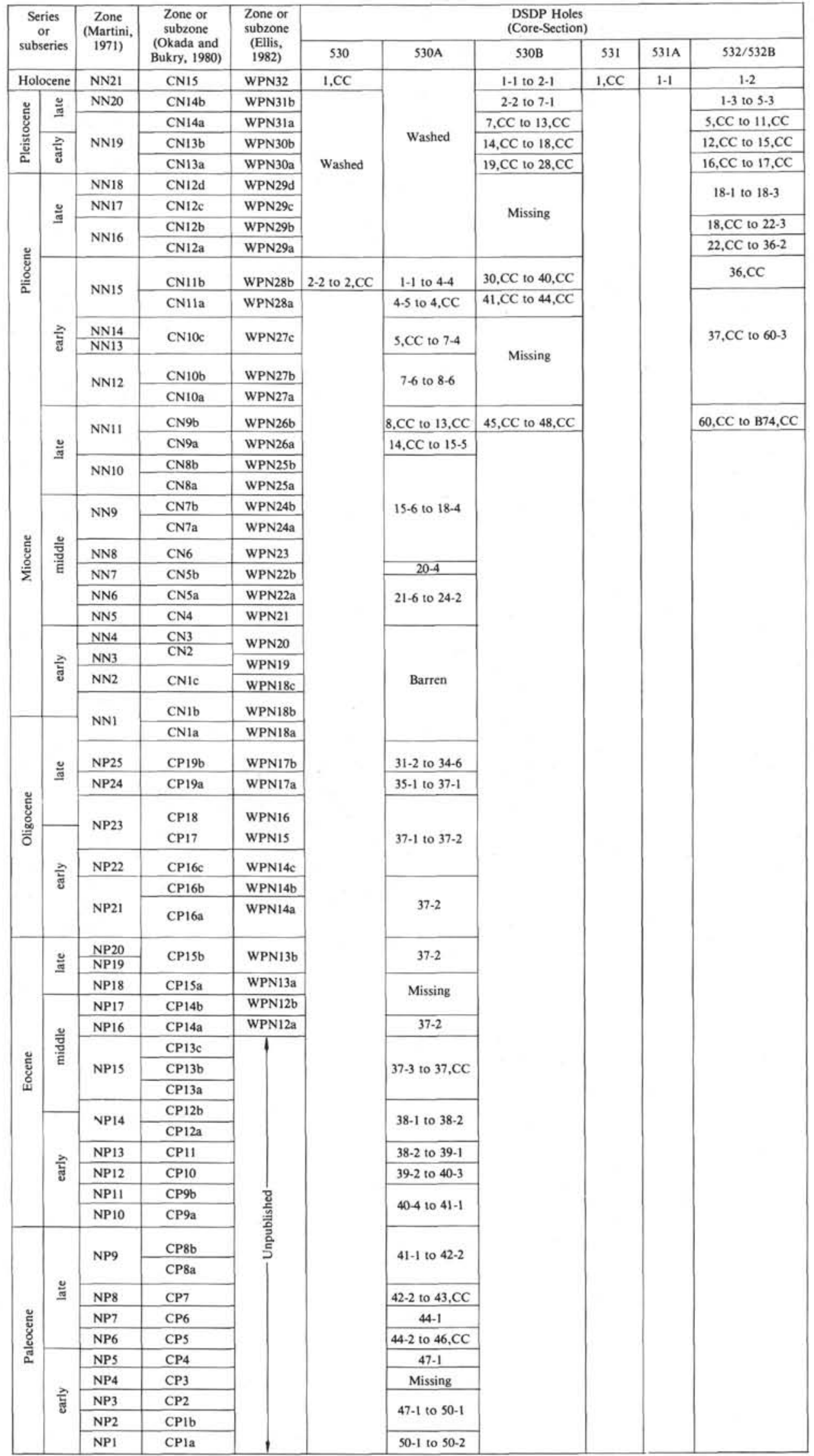




\section{NOTE ON THE PLATES}

The following abbreviations are used in the captions to the plates: NL = normal light; $(\mathrm{Al})=$ aluminum-shadowed; $(\mathrm{Au})=$ gold-shadowed $(\mathrm{RP})=$ Reverse print $\mathrm{PHC}=$ phase contrast $\mathrm{XN}=$ crossed nicols; $\mathrm{SEM}=$ scanning electron microscope (Elmi-Lab of the Geological Survey of Austria); and SEM* = scanning electron microscope (Electron Microscopy Facility of the University of South Florida).

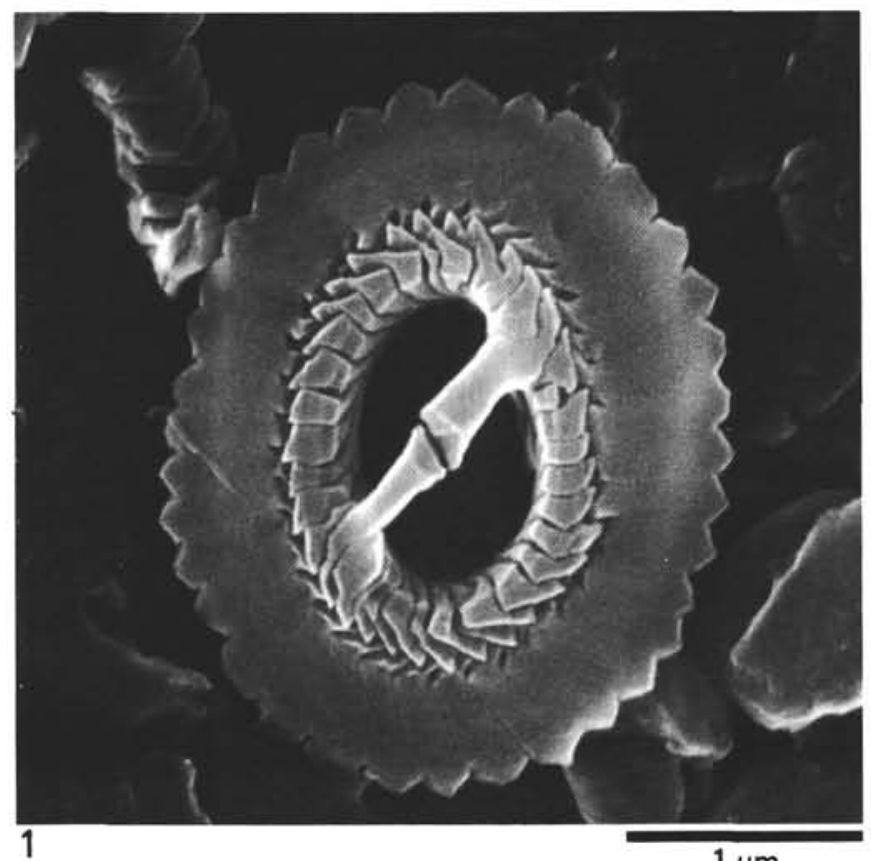

1

$1 \mu \mathrm{m}$
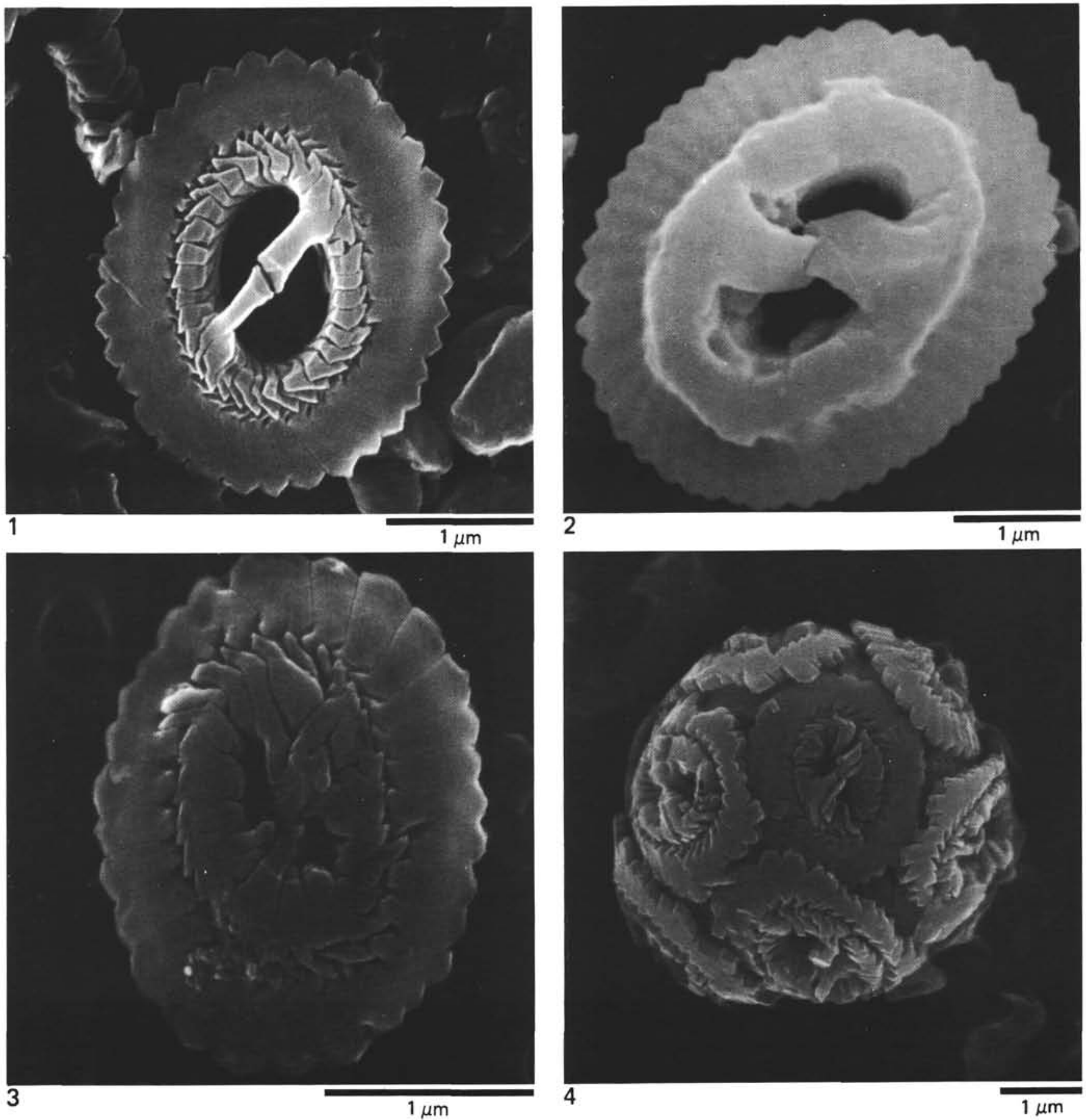

Plate 1. 1. Gephyrocapsa oceanica, Sample 530B-4,CC, SEM*; 2. G. caribbeanica, Sample 531A-1-1, 0-2 cm, SEM; 3. G. caribbeanica, Sample 532-5,CC, SEM*; 4. G. caribbeanica coccosphere, Sample 530B-4,CC, SEM*. 

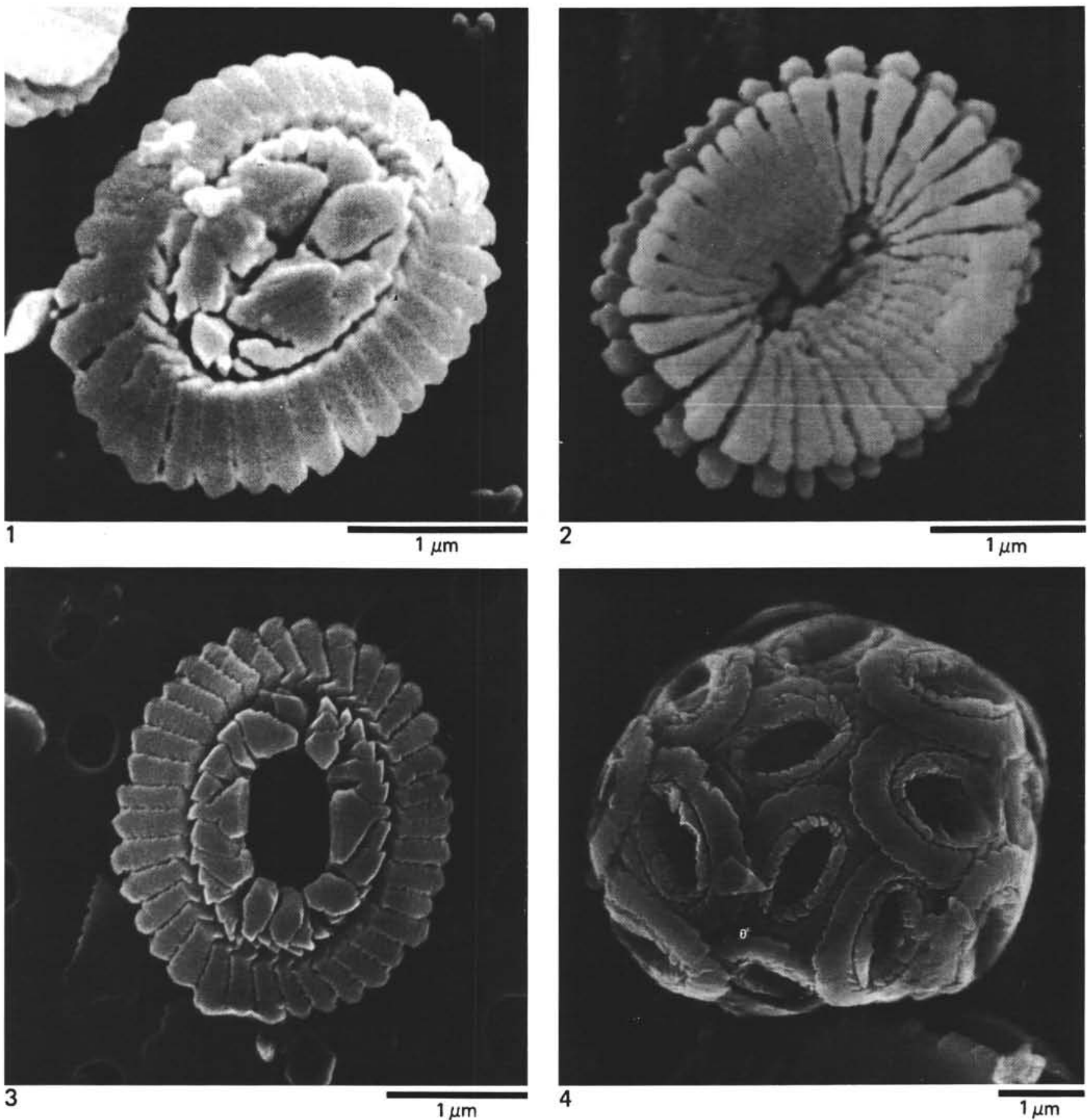

Plate 2. 1. Crenalithus productellus, distal view, Sample 532-44,CC, SEM; 2. C. productellus, proximal view, Sample 532-55,CC, SEM; 3. C. doronicoides, distal view, Sample 530B-29,CC, SEM; 4. C. doronicoides, coccosphere, Sample 530A-12-3-72/74 cm, SEM*. 

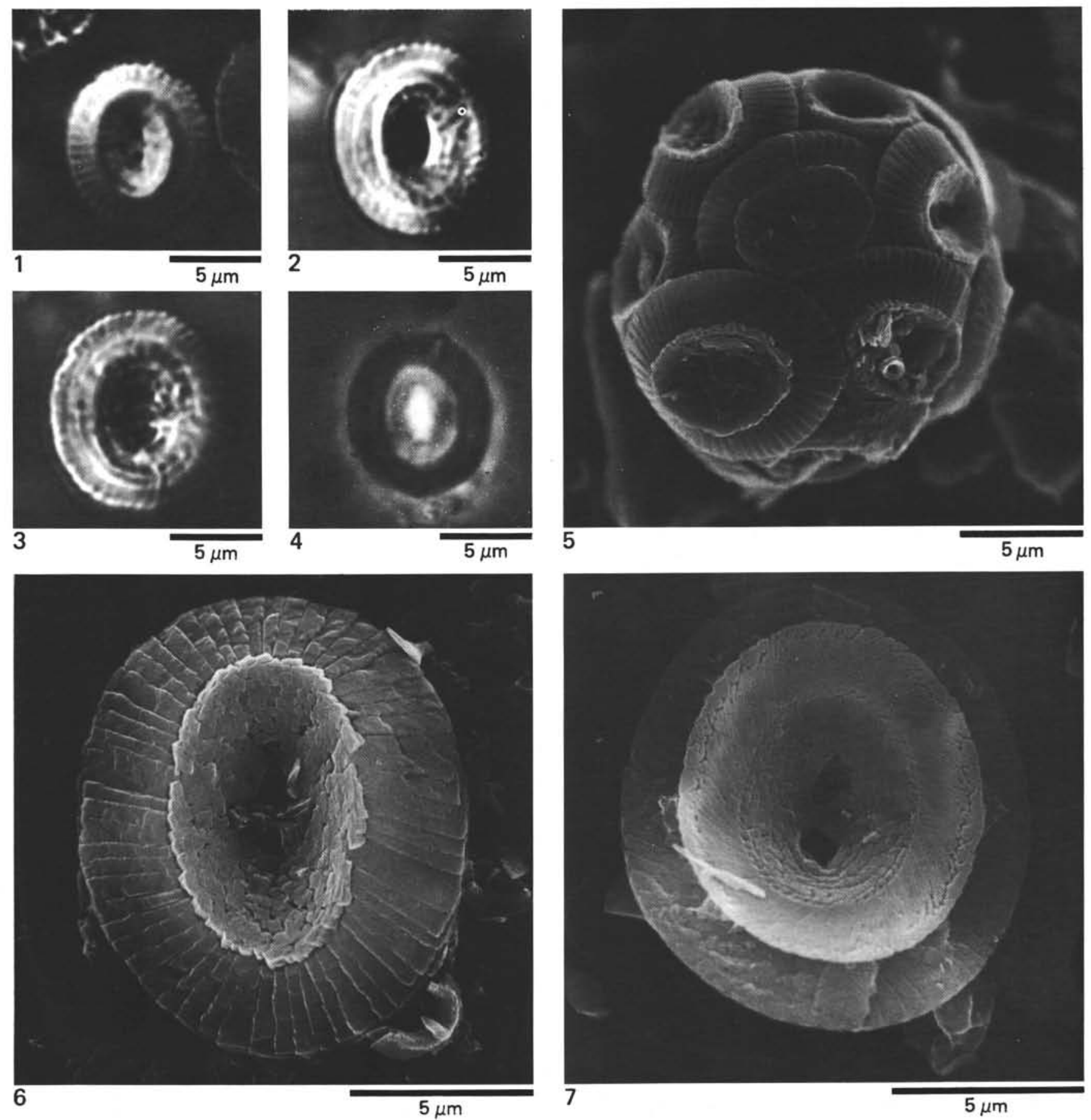

Plate 3. 1. Coccolithus carteri, Sample 531A-1-1, 0-2 cm, NL (Al, RP); 2. C. pelagicus, Sample 531A-1-1, 0-2 cm, NL (Al, RP); 3. C. carteri, Sample 531A-1-1, 0-2 cm, NL (AI, RP); 4. C. pelagicus, Sample 530A-35-1, 30-31 cm, PHC; 5. C. carteri coccosphere, Sample 530B-38,CC, SEM*; 6. C. carteri, distal view, Sample 530B-4,CC, SEM*; 7. C. carteri, proximal view, Sample 530B-4,CC, SEM*. 

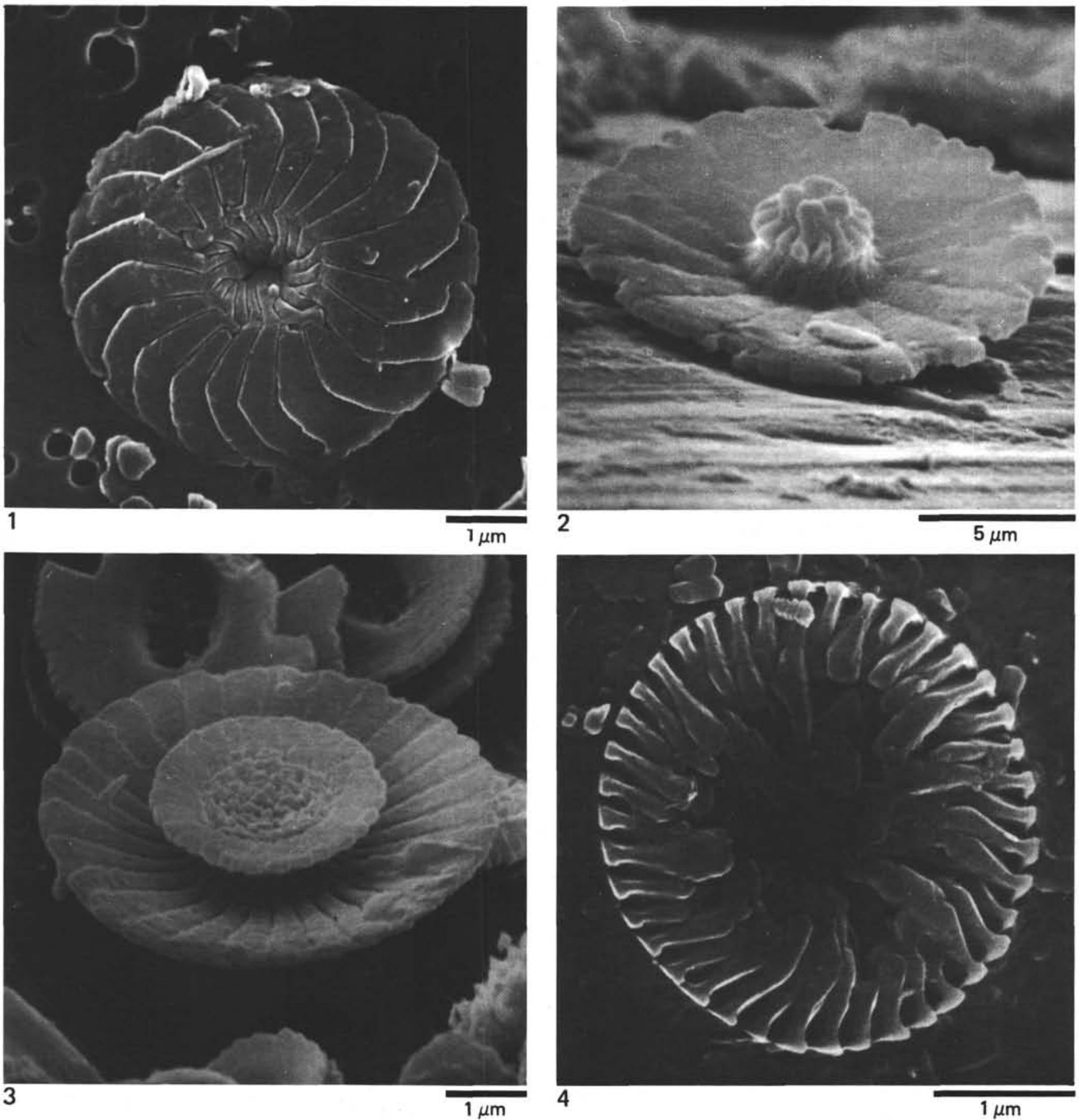

Plate 4. 1. Calcidiscus leptoporus, distal view, Sample 530B-39,CC, SEM*; 2. C. leptoporus, side view of proximal face, Sample 531A-1-1, 0-2 cm, SEM; 3. C. macintyrei, side view of proximal face, Sample 532-55,CC, SEM; 4. C. macintyrei, detail of center of proximal side of distal plate, Sample 532-5,CC, SEM*. 

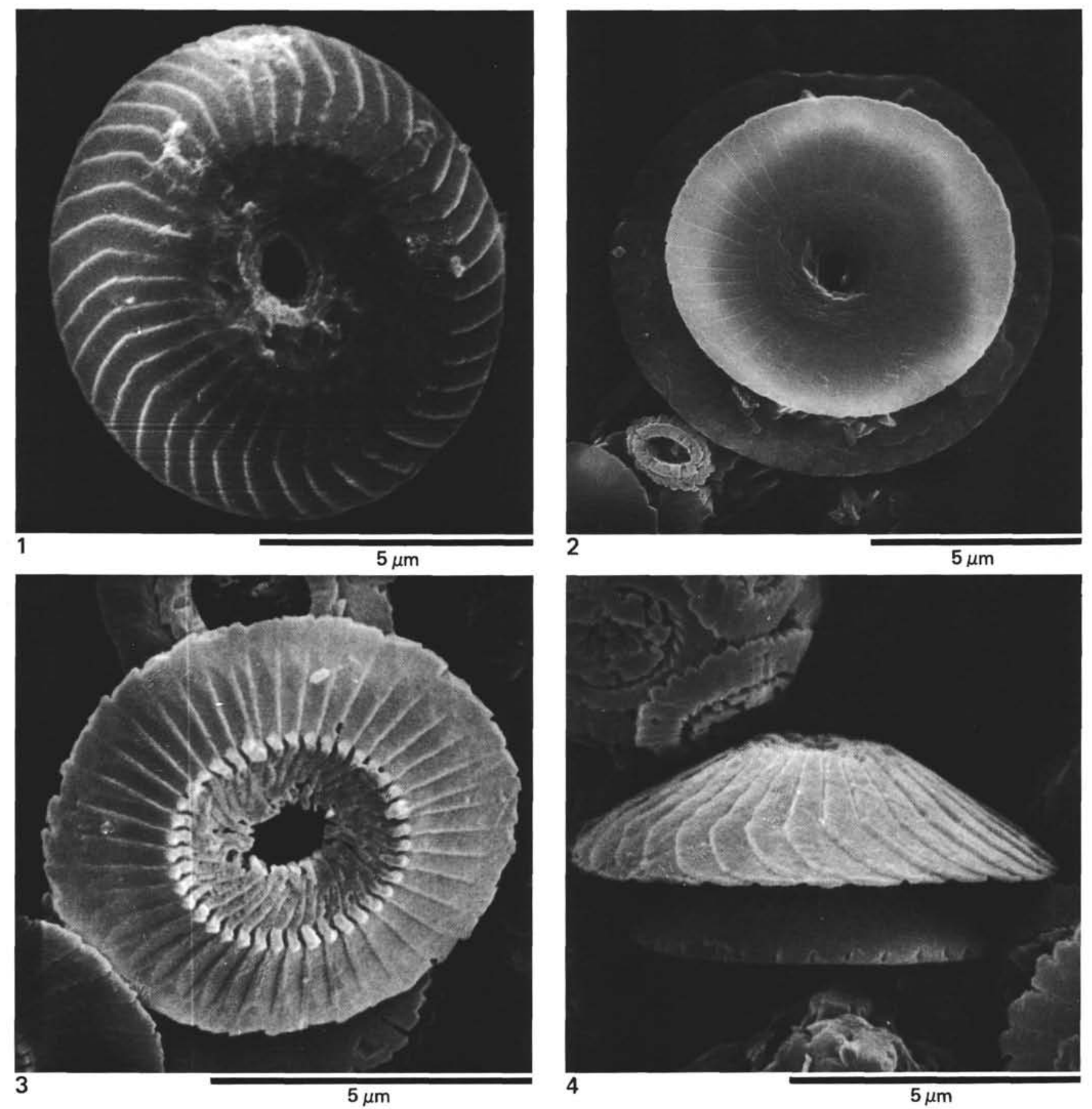

Plate 5. Calcidiscus macintyrei. 1. Distal view, Sample 532-36,CC, SEM; 2. Proximal view, Sample 530B-39,CC, SEM*; 3. Distal side of proximal plate, Sample 532-33,CC, SEM; 4. Side view, Sample 532-46,CC. 


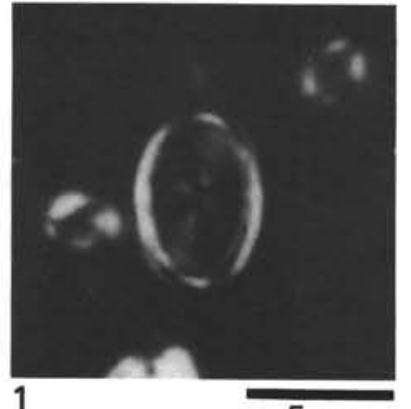

1

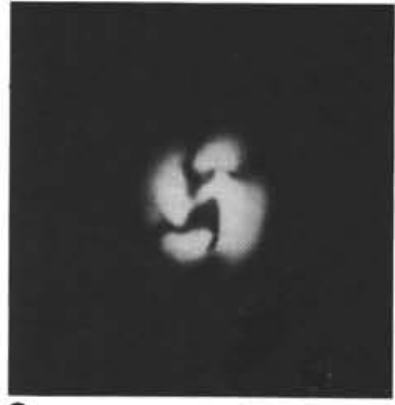

3
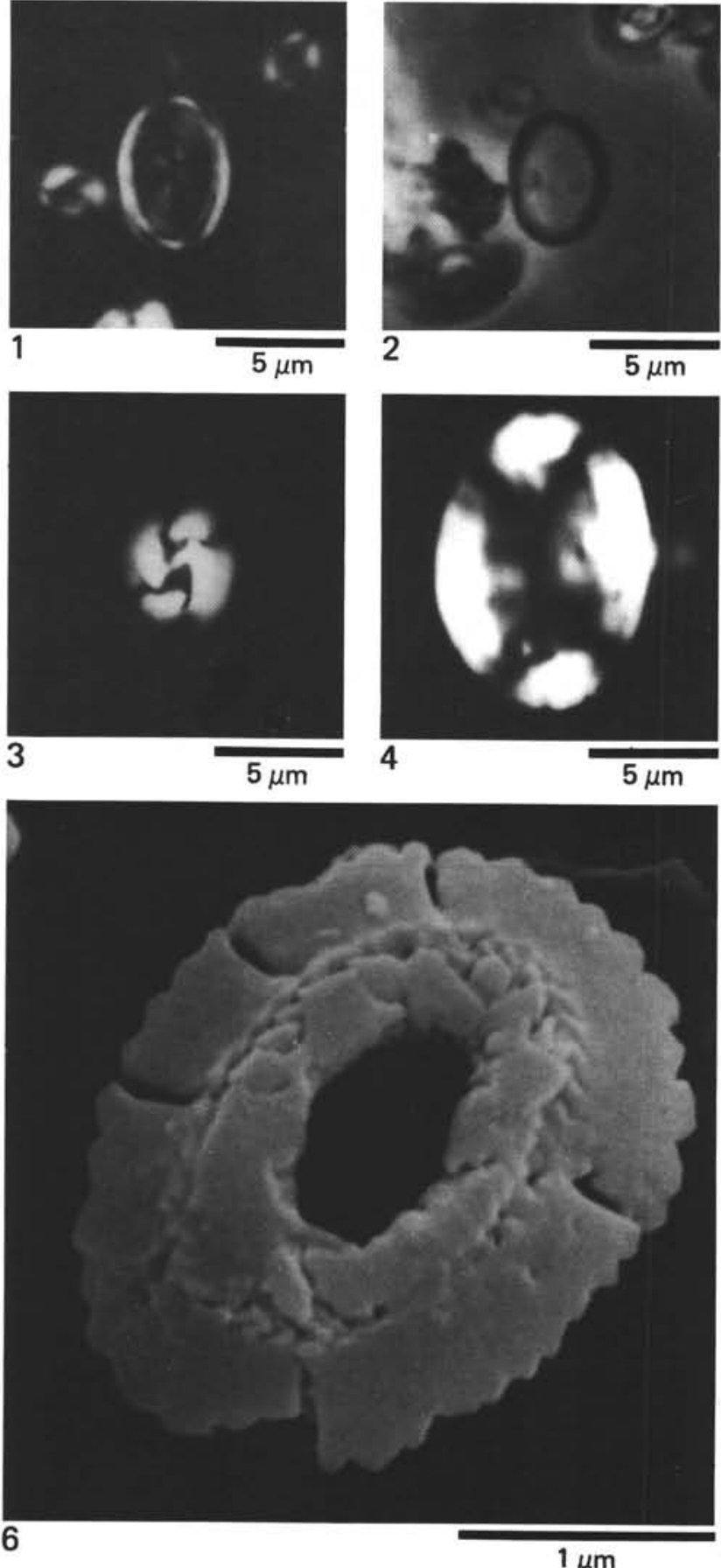

$5 \mu \mathrm{m}$
5
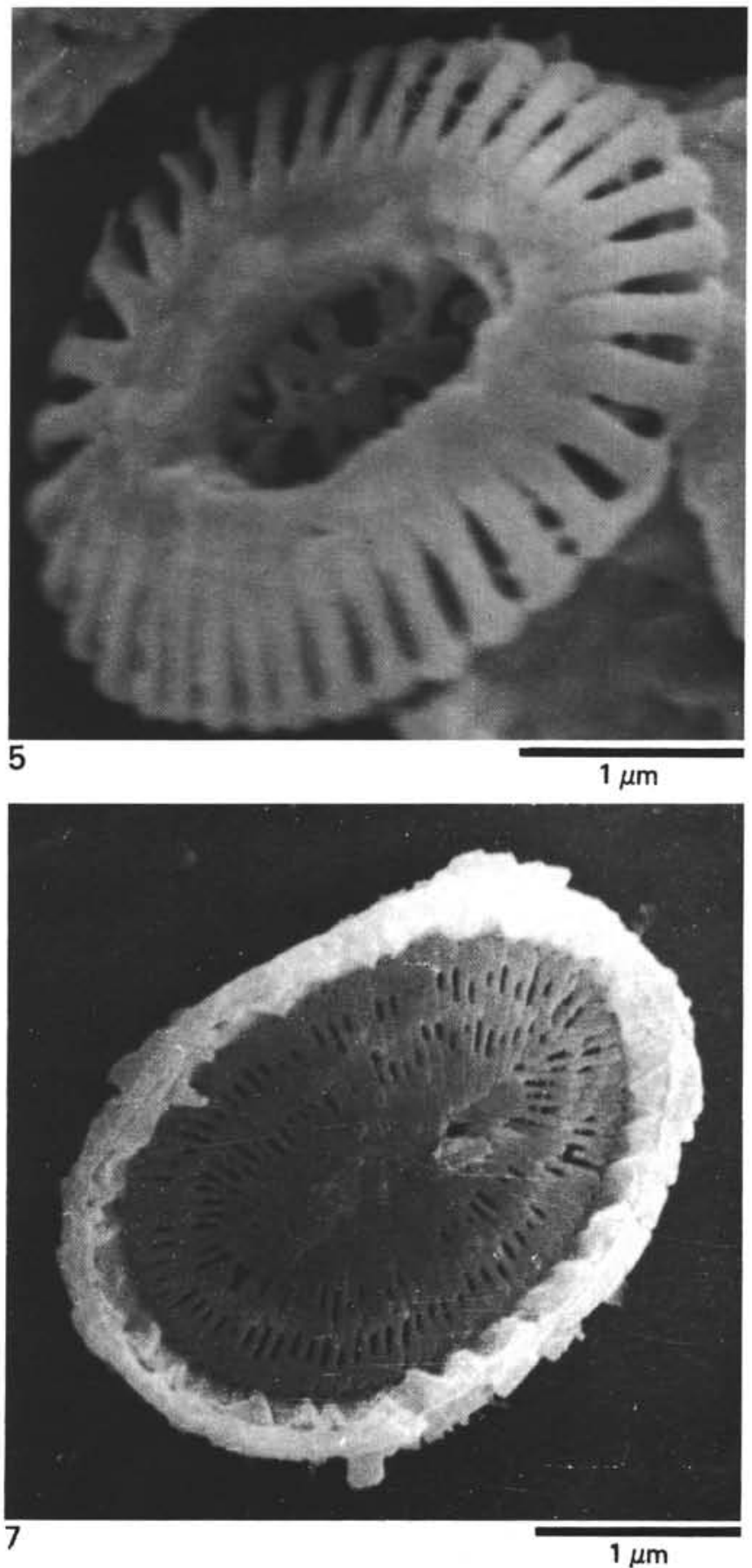

Plate 6. 1. Syracosphaera pulchra, Sample 531-1,CC, XN; 2. Syracosphaera cf. S. pulchra, Sample 531-1,CC, PHC; 3. Gephyrocapsa caribbeanica, Sample 531-1,CC, XN; 4. Pontosphaera syracusana, Sample 530A-1-1, 0-2 cm, XN; 5. Emiliania huxleyi, distal view, Sample 531A-1-1, 0-2 cm, SEM; 6. Crenalithus doronicoides, distal view, Sample 532-36,CC, SEM; 7. Syracosphaera pulchra, distal view, Sample $531 \mathrm{~A}-1-1,0-2 \mathrm{~cm}$, SEM. 


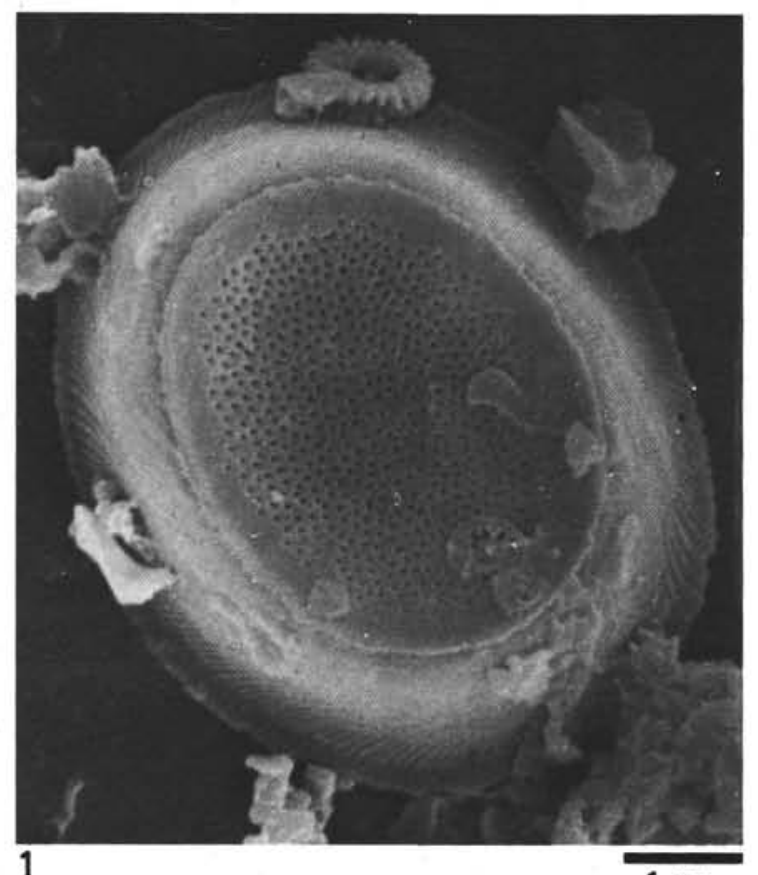

1

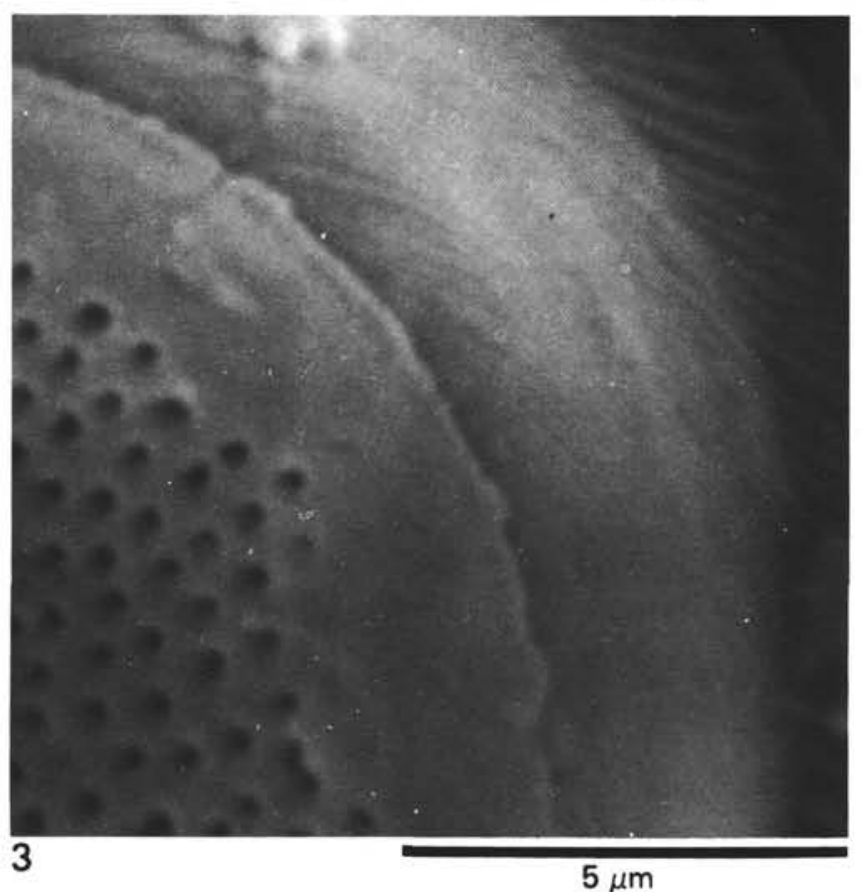

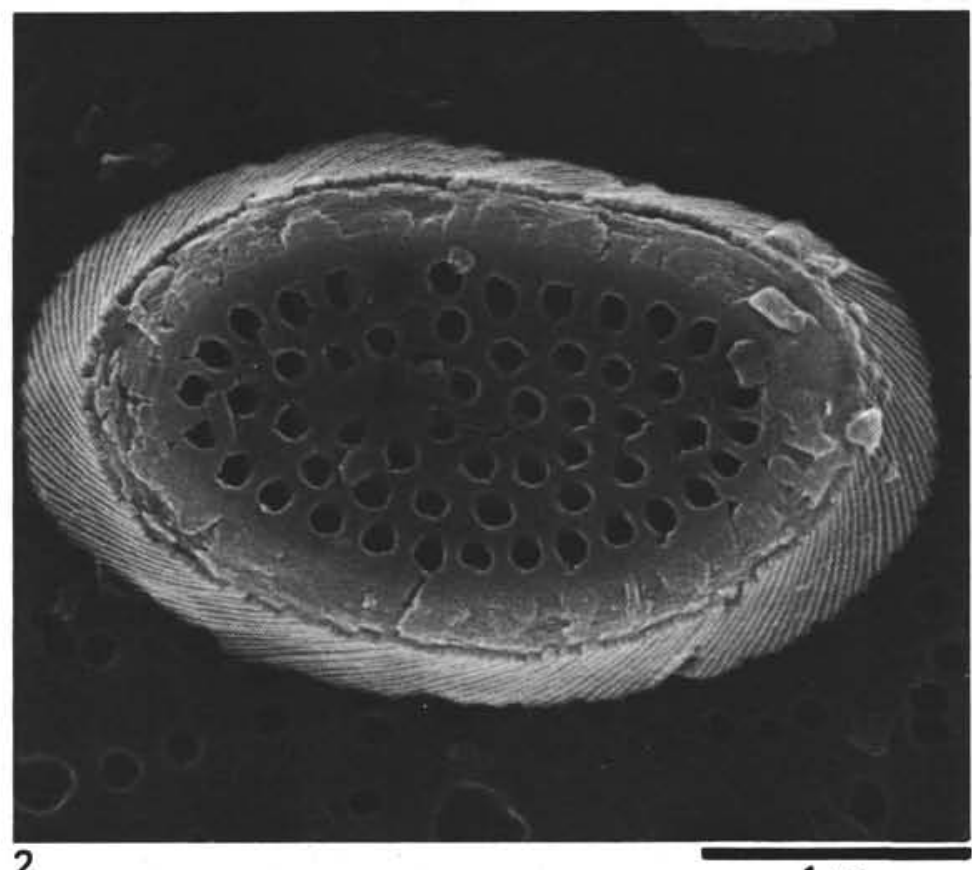

$1 \mu \mathrm{m}$

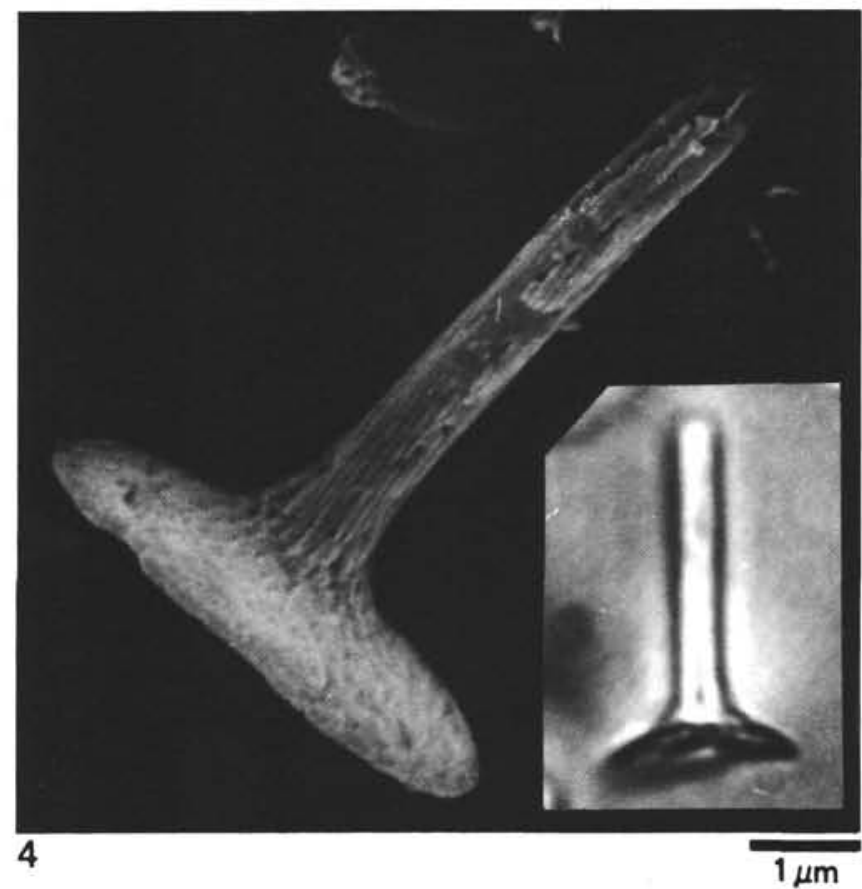

Plate 7. 1. Pontosphaera alboranensis, proximal view, Sample $531 \mathrm{~A}-1-1,0-2 \mathrm{~cm}$, SEM; 2. Pontosphaera $\mathrm{cf}$. $P$. multipora, proximal view, Sample 530B-29,CC, SEM*; 3. P. alboranensis, detail of Figure 1, SEM; 4. Rhabdosphaera sicca, side view, Sample 532-46,CC, SEM (inset, NL). 

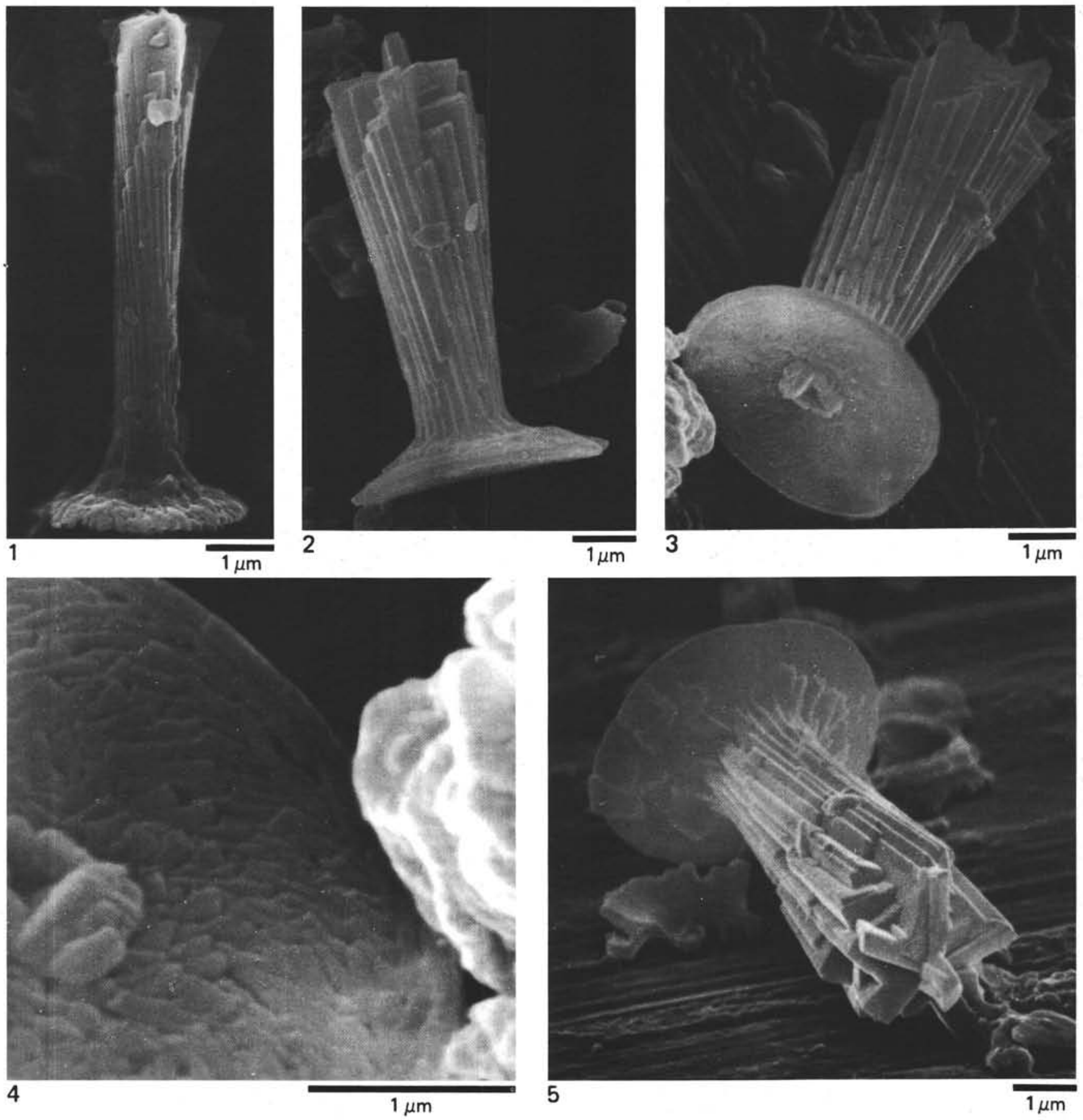

Plate 8. Rhabdosphaera clavigera. 1. Side view, Sample 530B-39,CC, SEM*; 2. Side view, Sample 531A-1-1, 0-2 cm, SEM. 3. Oblique proximal view, Sample 531A-1-1, 0-2 cm, SEM; 4. Proximal view of basal plate, Sample 531A-1-1, 0-2 cm, SEM; 5. Distal view, Sample $531 \mathrm{~A}-1-1,0-2 \mathrm{~cm}$, SEM. 

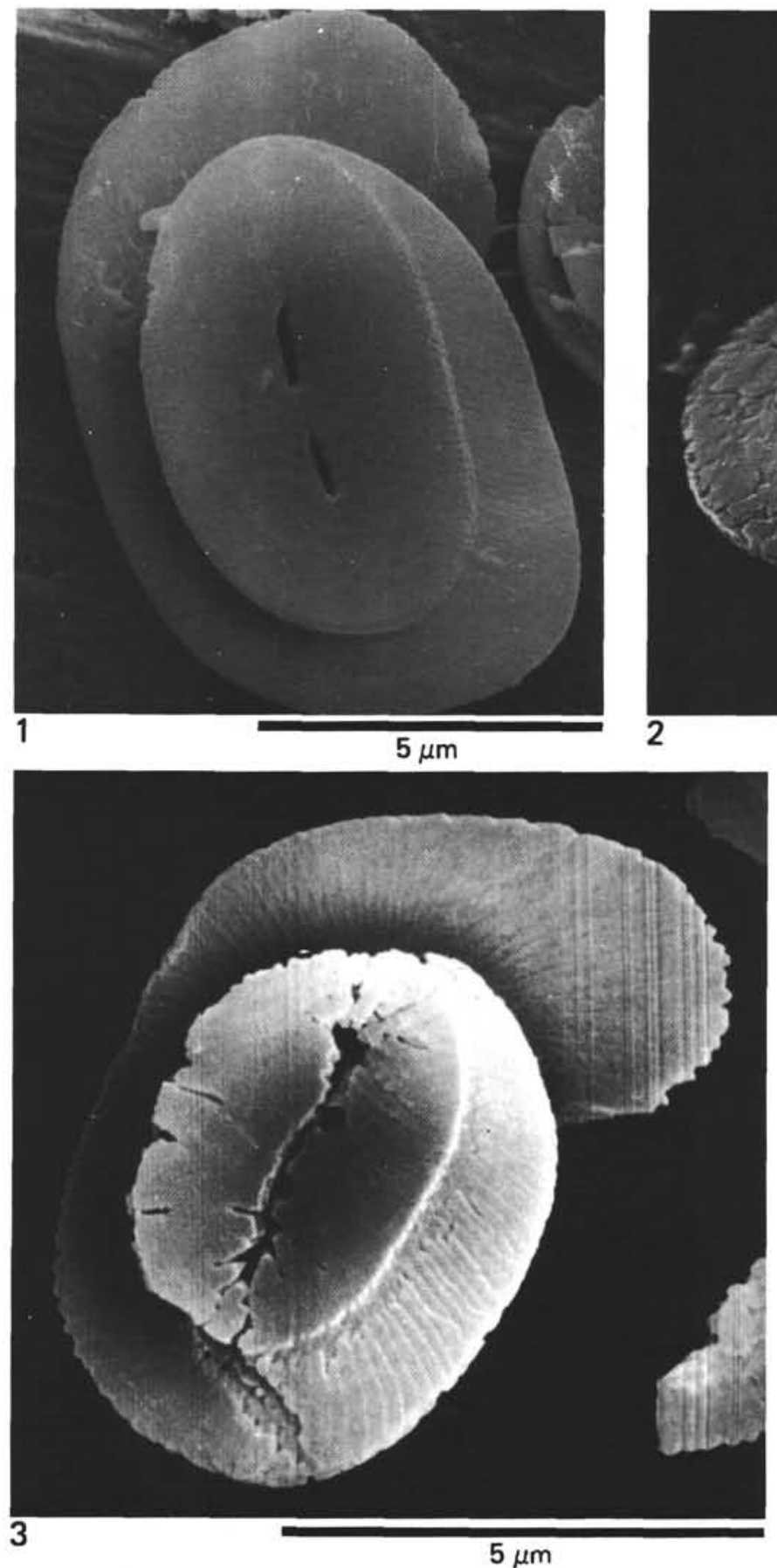

$5 \mu \mathrm{m}$

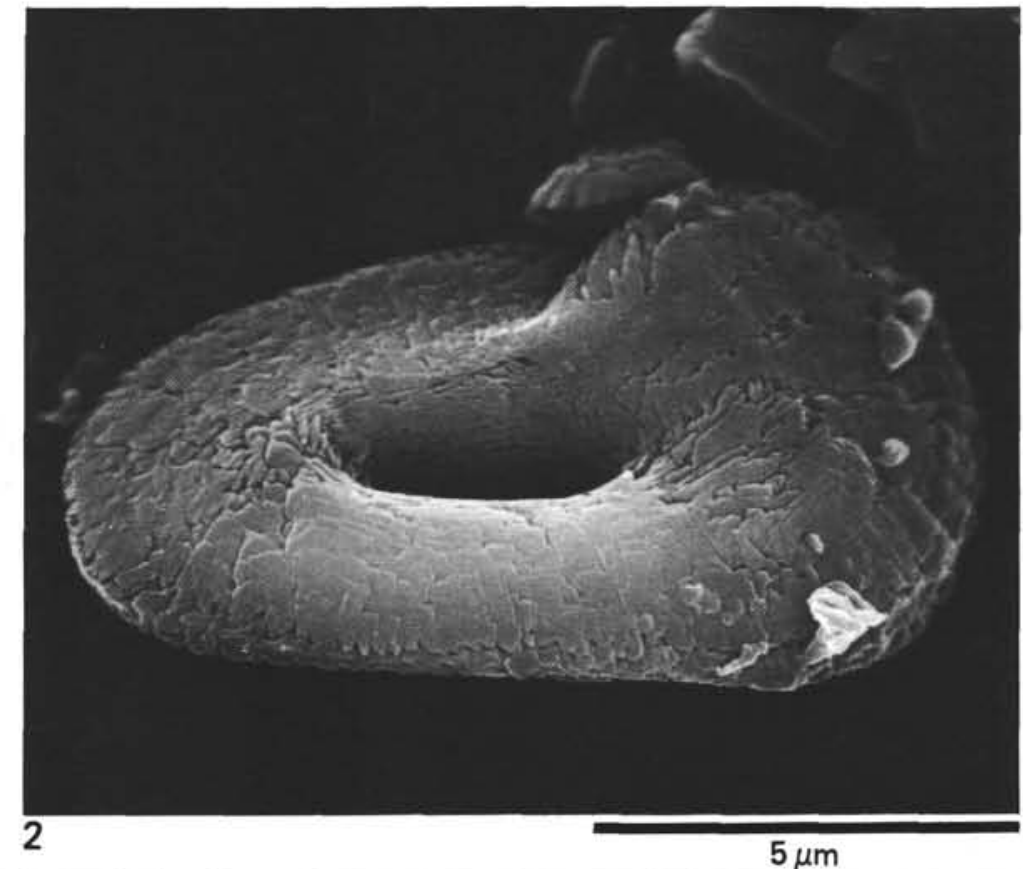

$5 \mu \mathrm{m}$

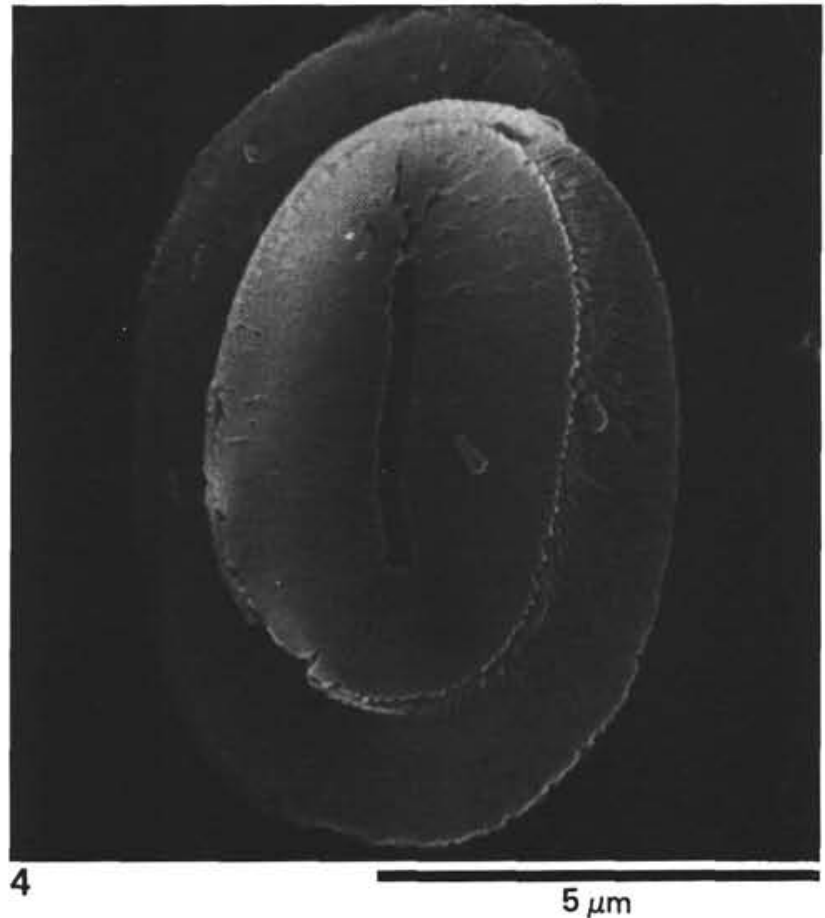

$5 \mu \mathrm{m}$

Plate 9. Helicosphaera carteri. 1. Proximal view, Sample 531A-1-1, 0-2 cm, SEM; 2. Distal view, Sample 530B-29,CC, SEM*; 3. Proximal view, Sample 532-55,CC, SEM; 4. Proximal view, Sample 530B-29, CC, SEM*. 


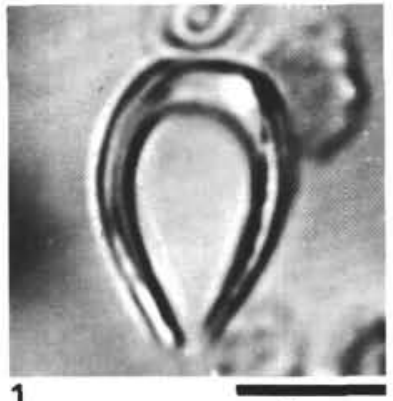

1

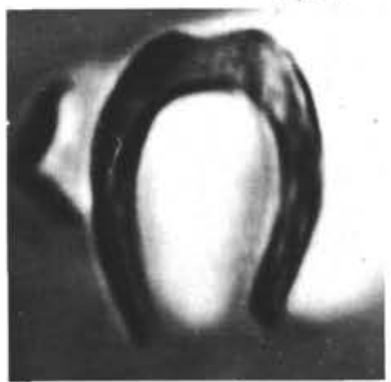

3

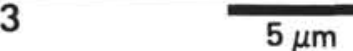

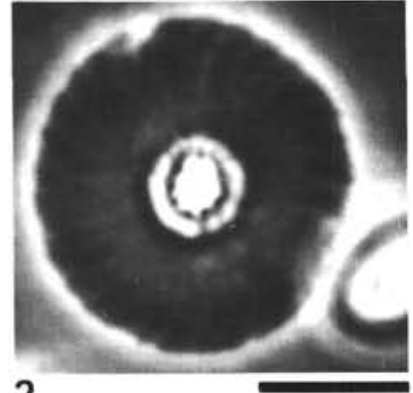

$5 \mu \mathrm{m}$

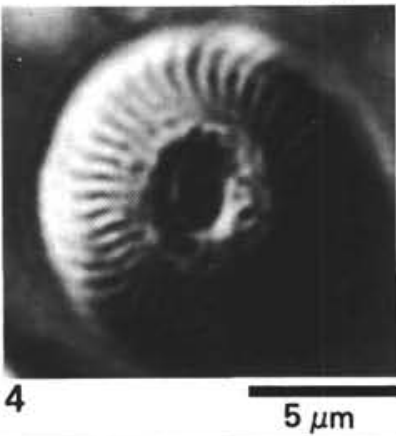

$5 \mu \mathrm{m}$

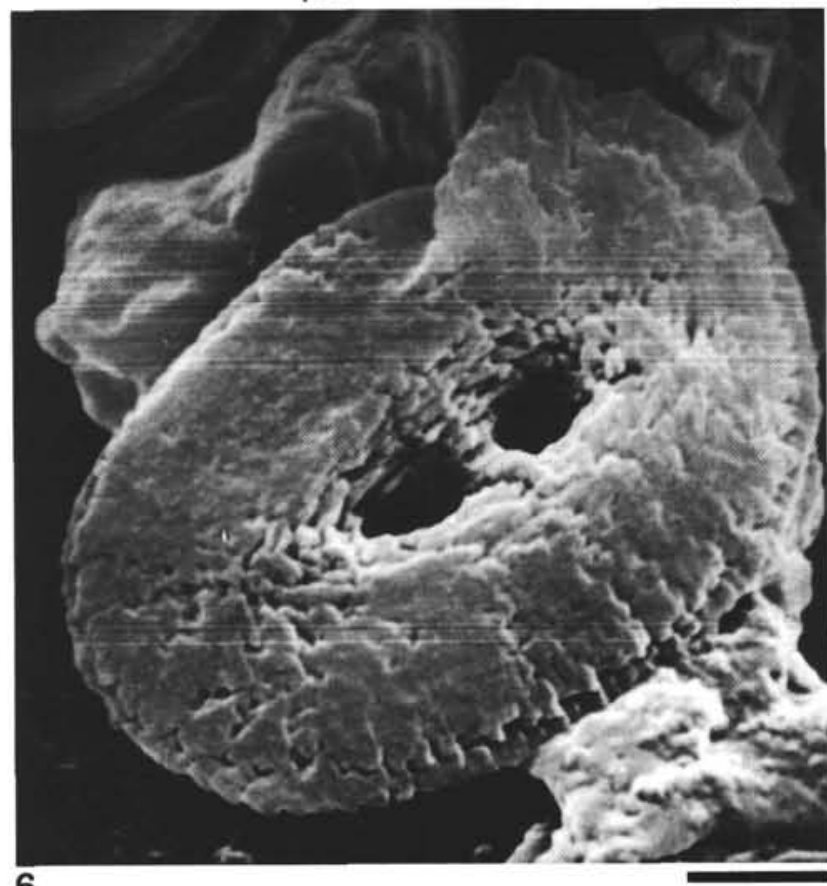

$1 \mu \mathrm{m}$

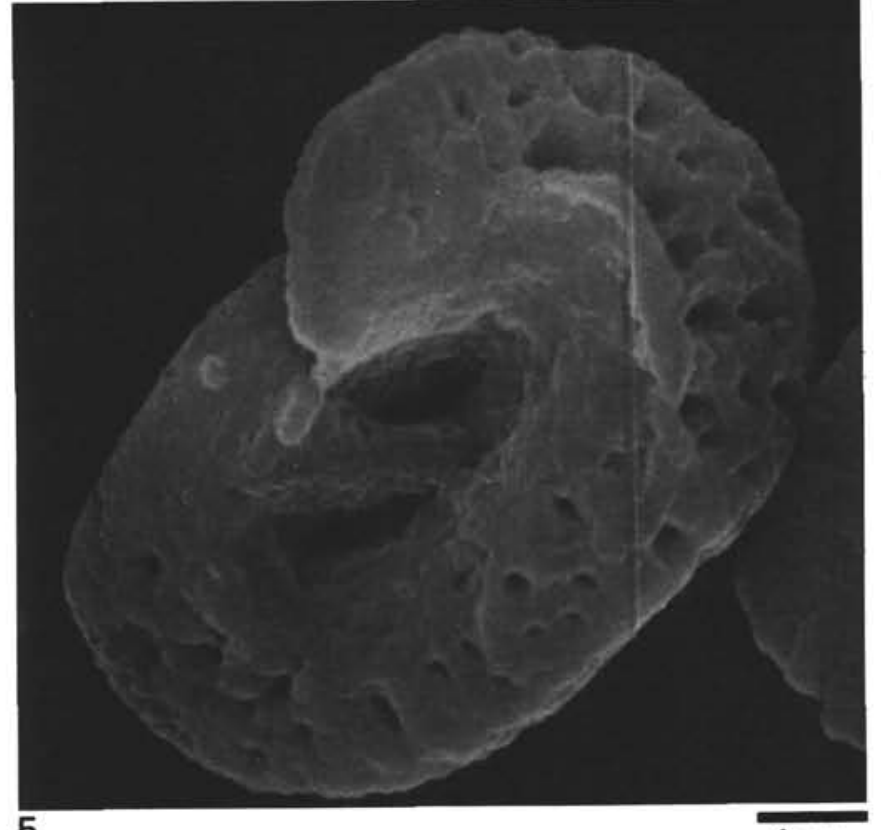

$1 \mu \mathrm{m}$

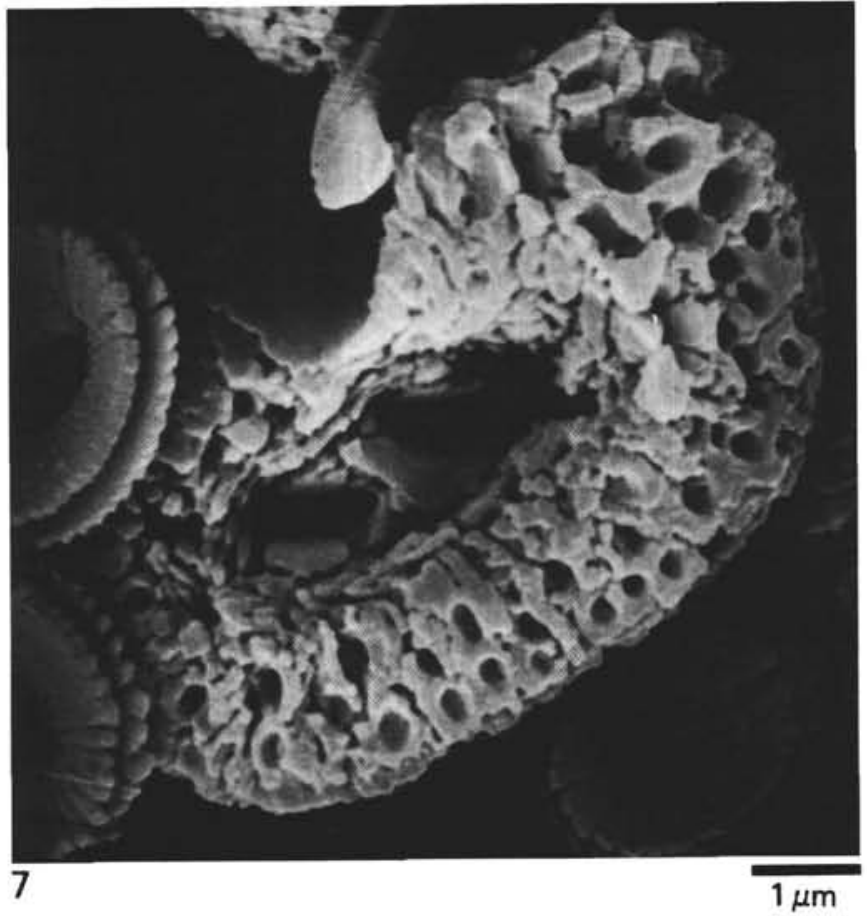

Plate 10. 1. Amaurolithus delicatus, Sample 532-55,CC, NL.; 2. Calcidiscus macintyrei, Sample 532-33,CC, PHC; 3. A. delicatus, Sample 532-55, CC, NL (Al); 4. C. macintyrei, Sample 532-33,CC, NL (Al, RP); 5-7. Helicosphaera sellii, distal view, etched, Sample 532-55,CC, SEM. 

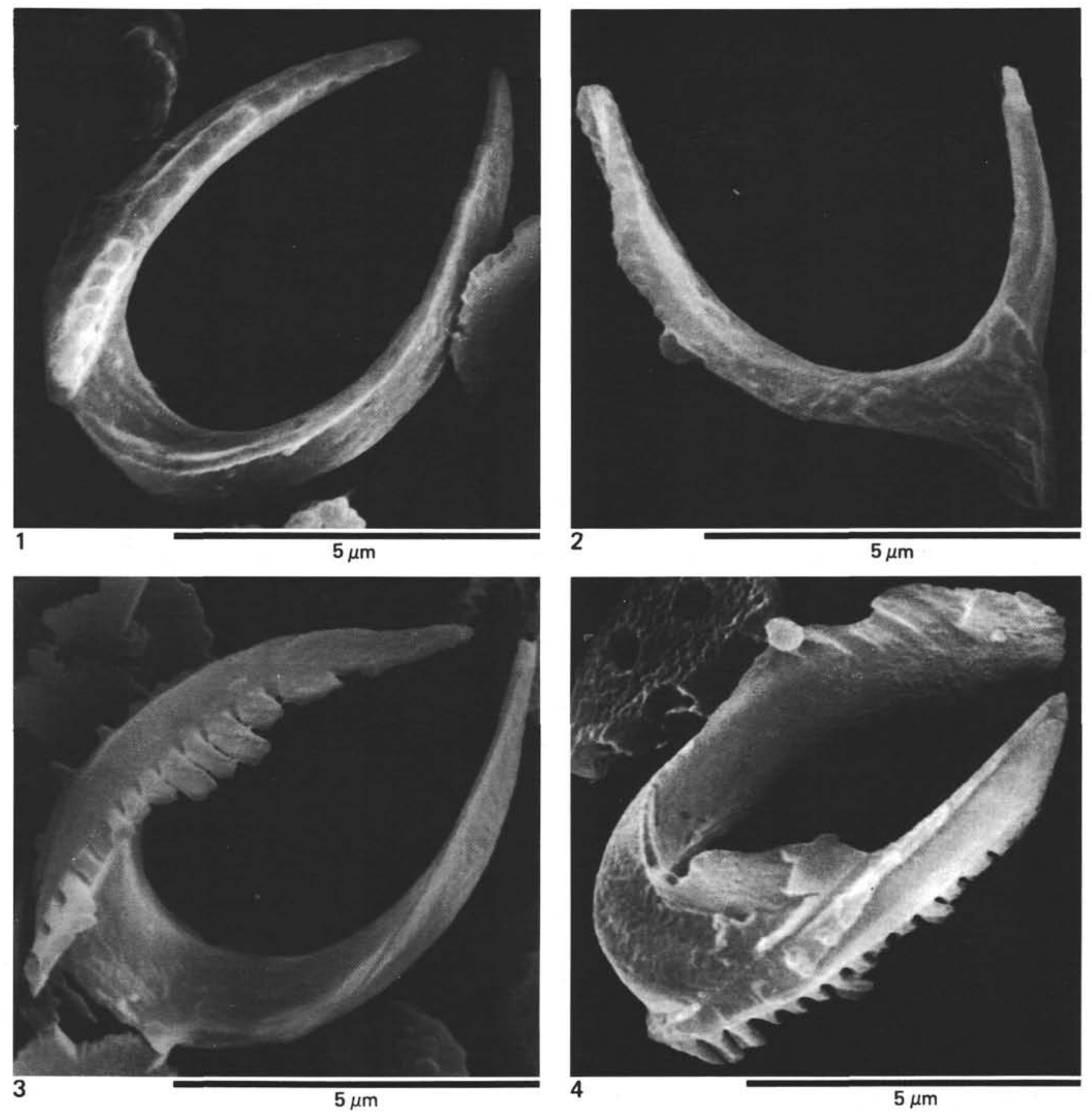

Plate 11. 1. Amaurolithus delicatus, Sample 532-46,CC, SEM; 2. A. tricorniculatus, Sample 532-55,CC, SEM; 3. A. tricorniculatus, Sample 532-46,CC, SEM; 4. A. cf. A. tricorniculatus, Sample 532-55,CC, SEM. 


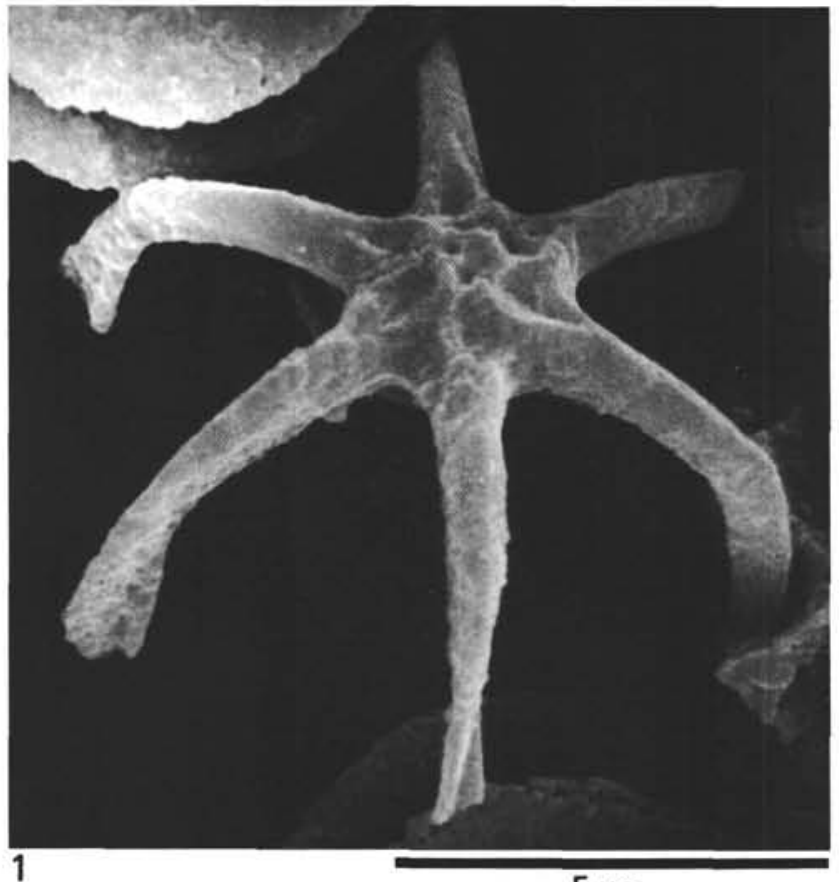

$5 \mu \mathrm{m}$

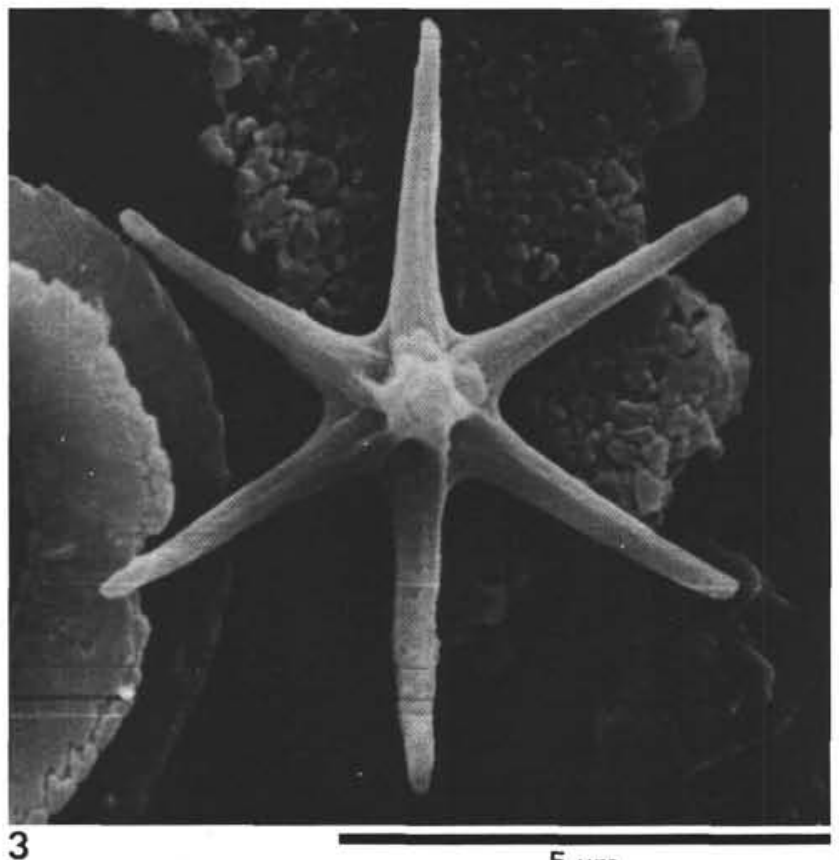

$5 \mu \mathrm{m}$
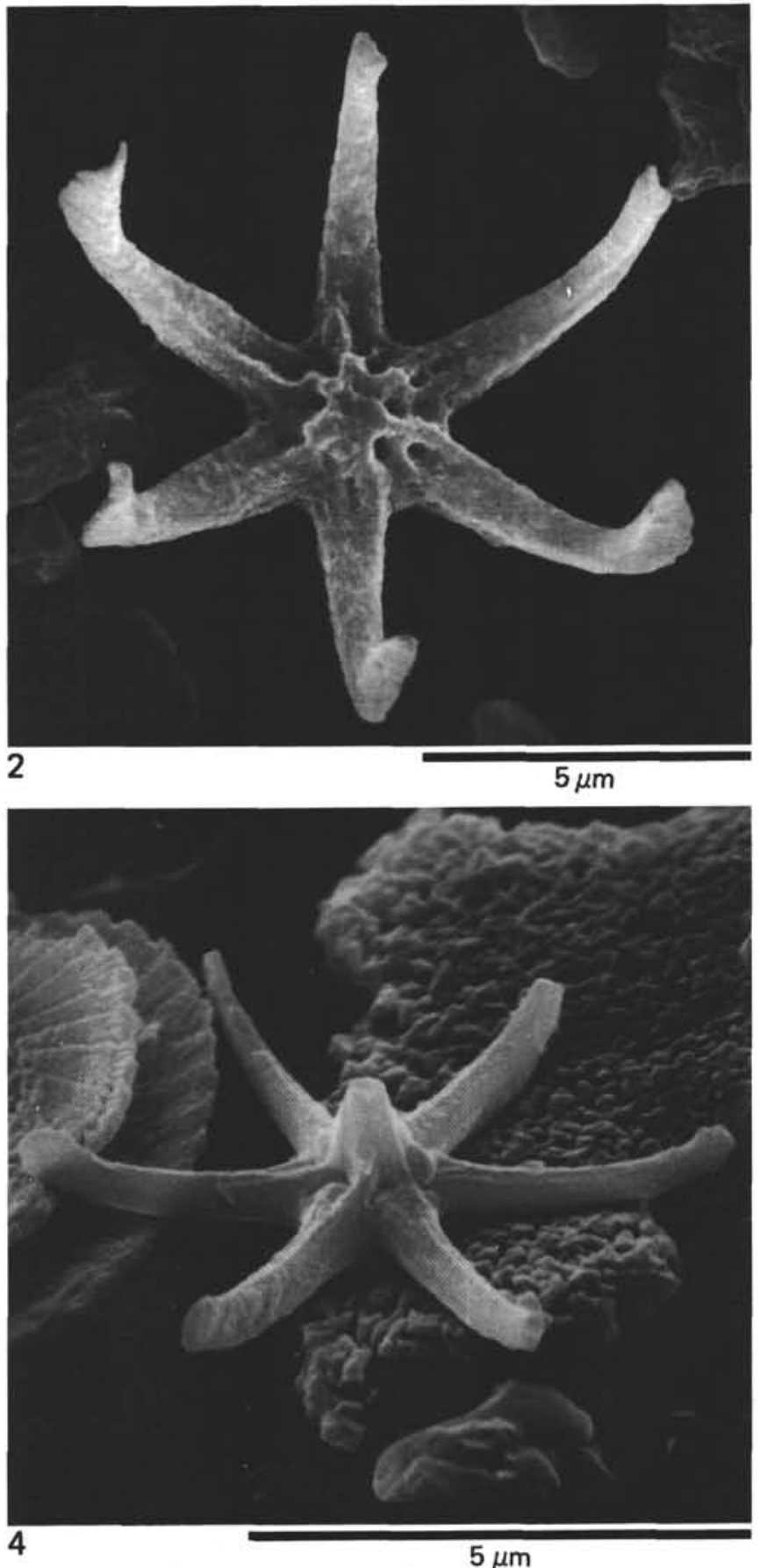

Plate 12. Discoaster brouweri. 1. Distal view, Sample 532-36,CC, SEM; 2-3. Proximal view, Sample 532-55,CC, SEM; 4. Oblique proximal view, Sample 532-55,CC, SEM. 

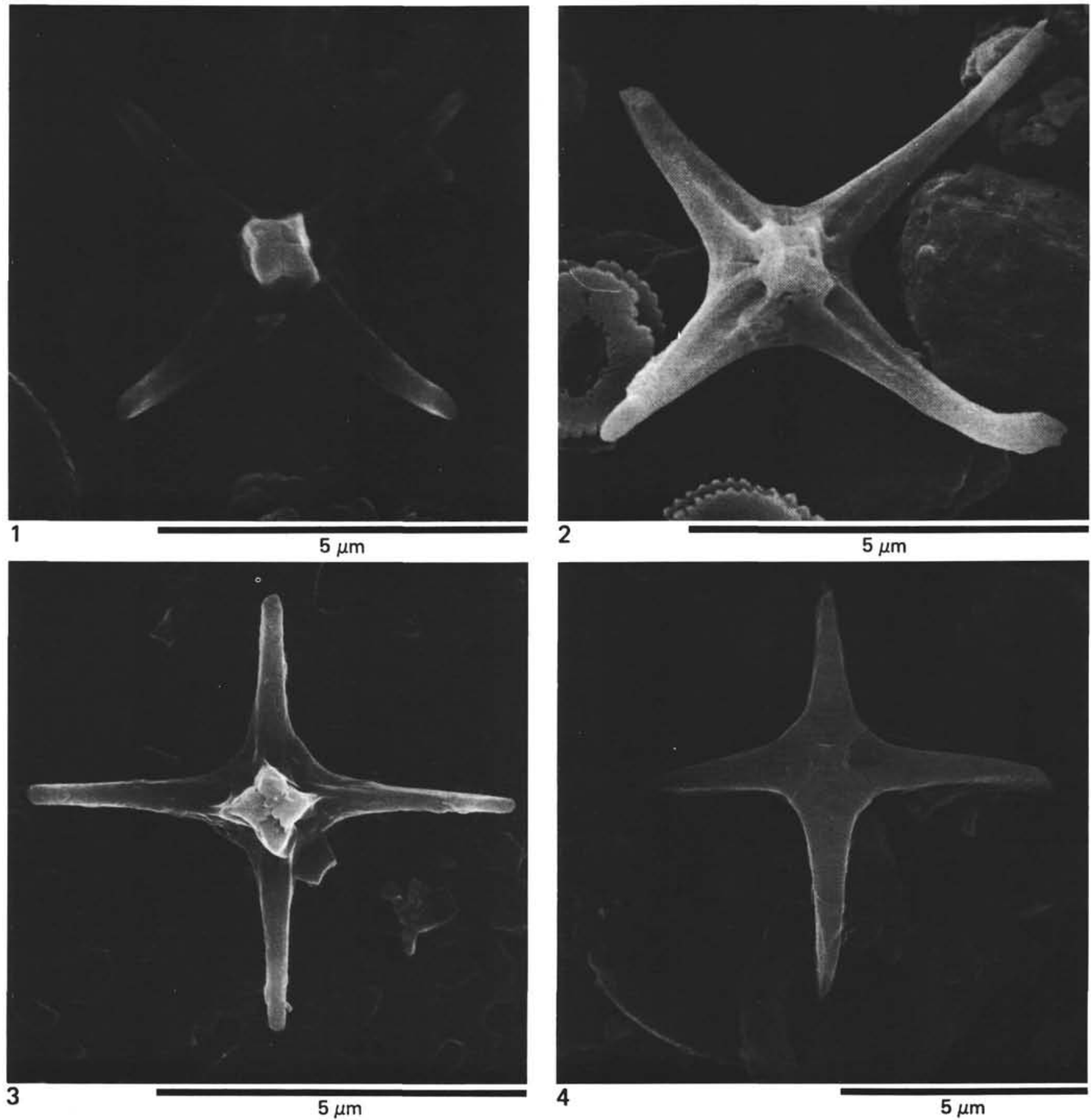

Plate 13. Discoaster tamalis. 1. Proximal view, Sample 530B-38,CC, SEM*; 2. Proximal view, Sample 532-36,CC, SEM; 3. Proximal view, Sample 532-5,CC, SEM*; 4. Distal view, Sample 530B-38,CC, SEM*. 


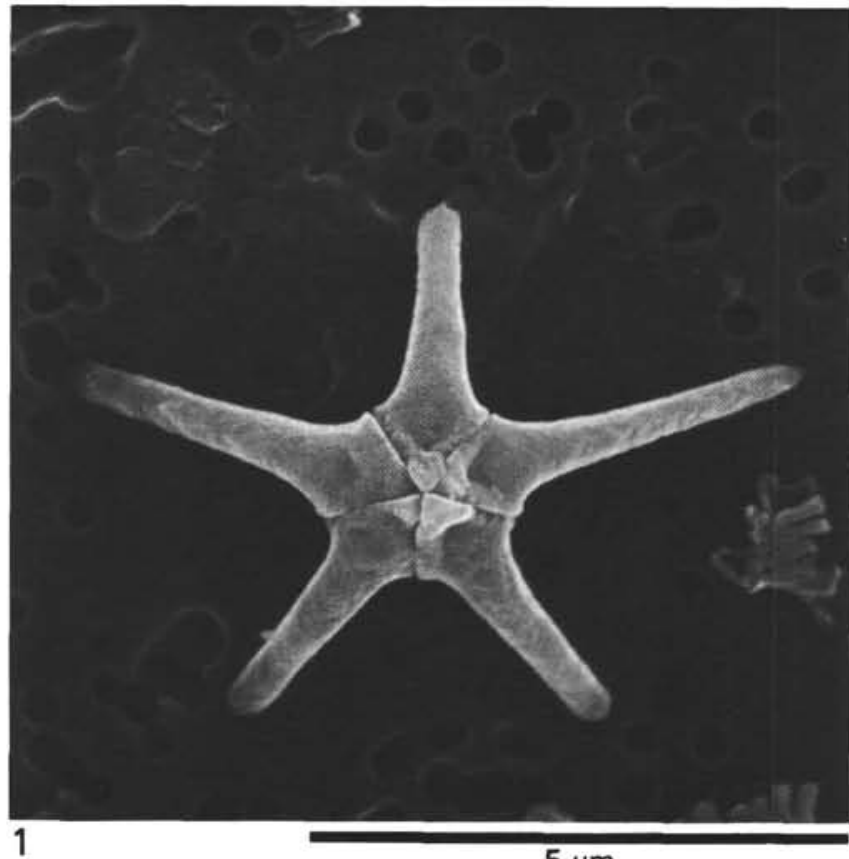

$5 \mu \mathrm{m}$

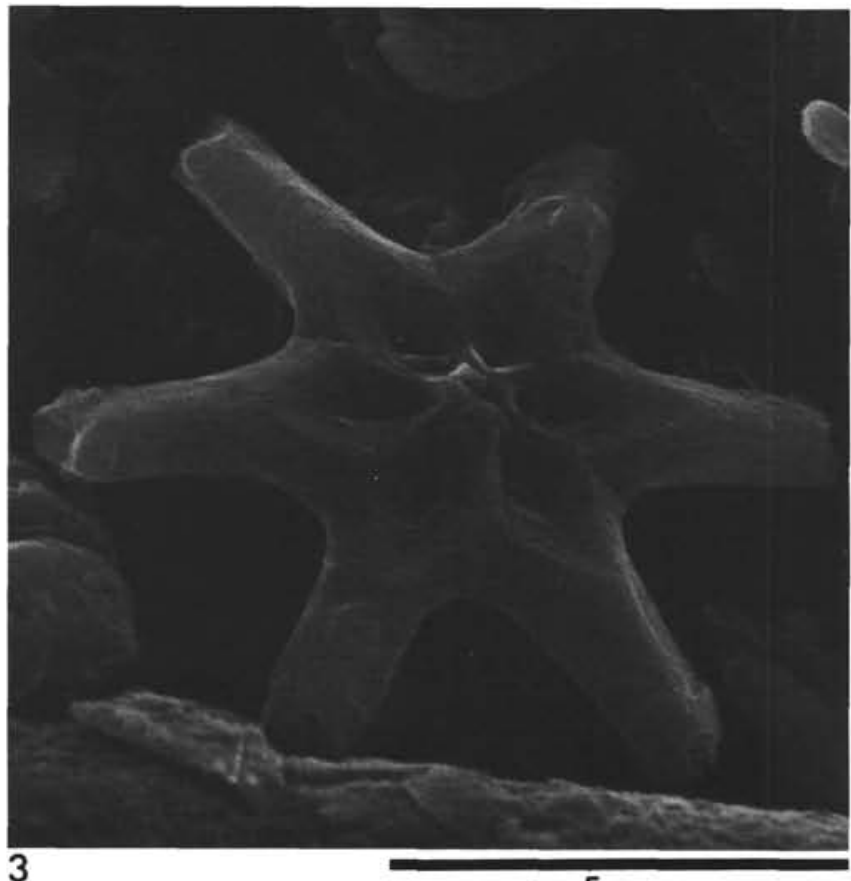

$5 \mu \mathrm{m}$
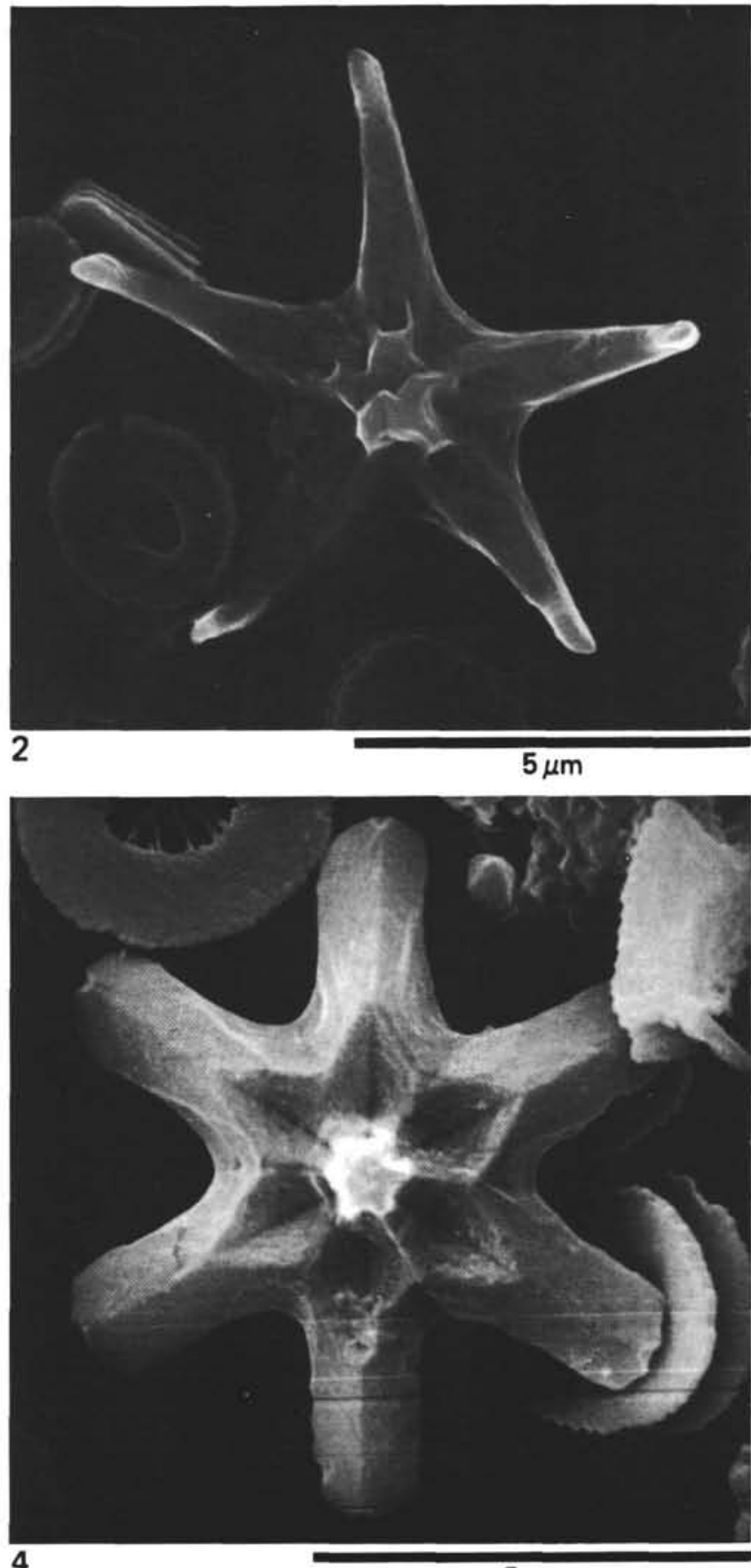

$5 \mu \mathrm{m}$

Plate 14. 1. Discoaster cf. D. pentaradiatus, distal view, Sample 530B-29,CC, SEM*; 2. Discoaster cf. D. pentaradiatus, proximal view, Sample 532-5,CC, SEM*; 3. D. intercalcaris, distal view, Sample 530B-38,CC, SEM*; 4. D. intercalcaris, distal view, Sample 532-55,CC, SEM. 

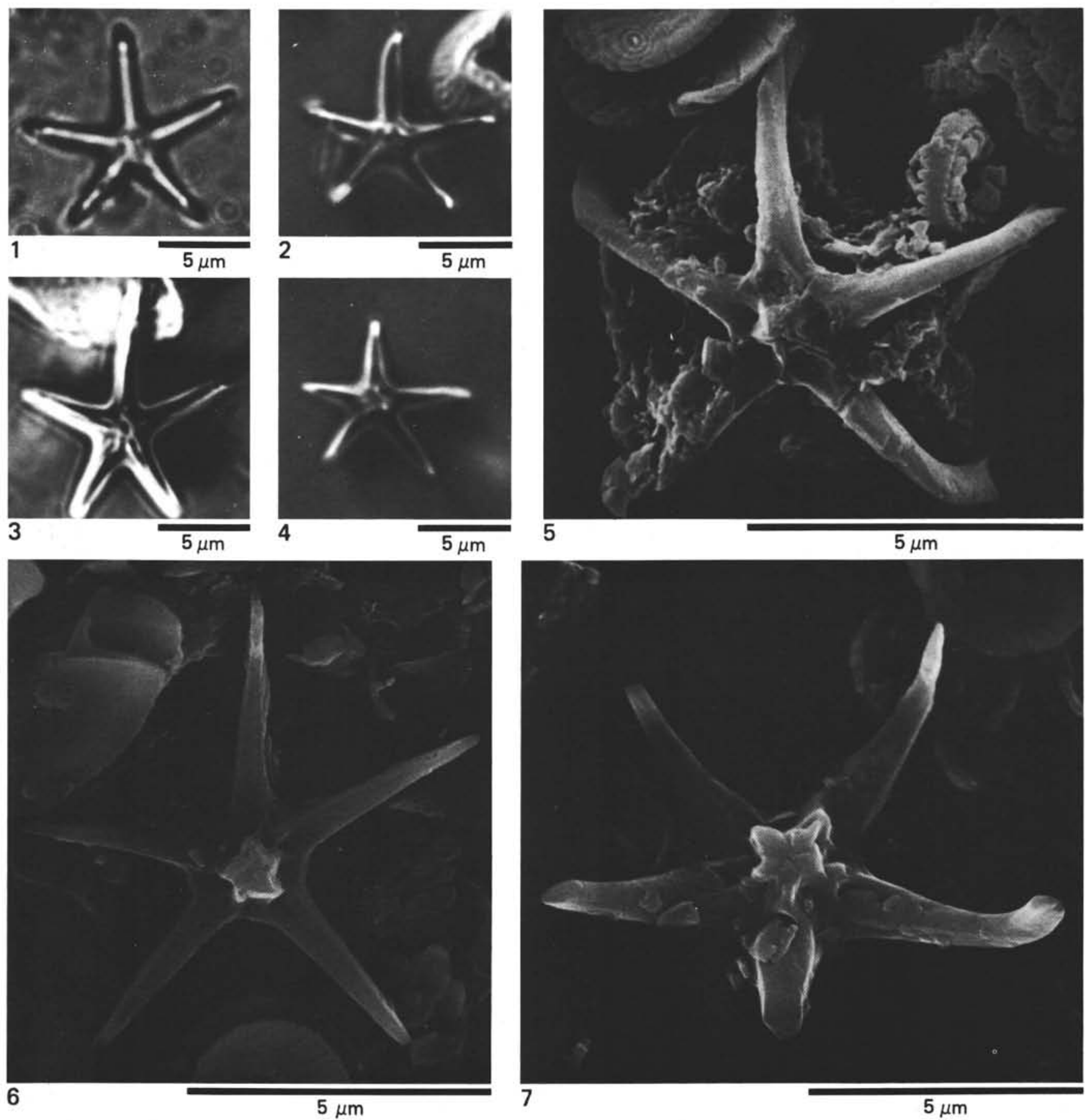

Plate 15. 1. Discoaster asymmetricus, Sample 532-33,CC, NL; 2-4. D. asymmetricus, Sample 532-33, CC, NL (Al); 5. D. pentaradiatus, distal view, Sample 532-33,CC, SEM; 6. Discoaster asymmetricus, proximal view, Sample 530B-38,CC, SEM*; 7. Discoaster cf. D. asymmetricus, proximal view, Sample 530B-38,CC, SEM*. 

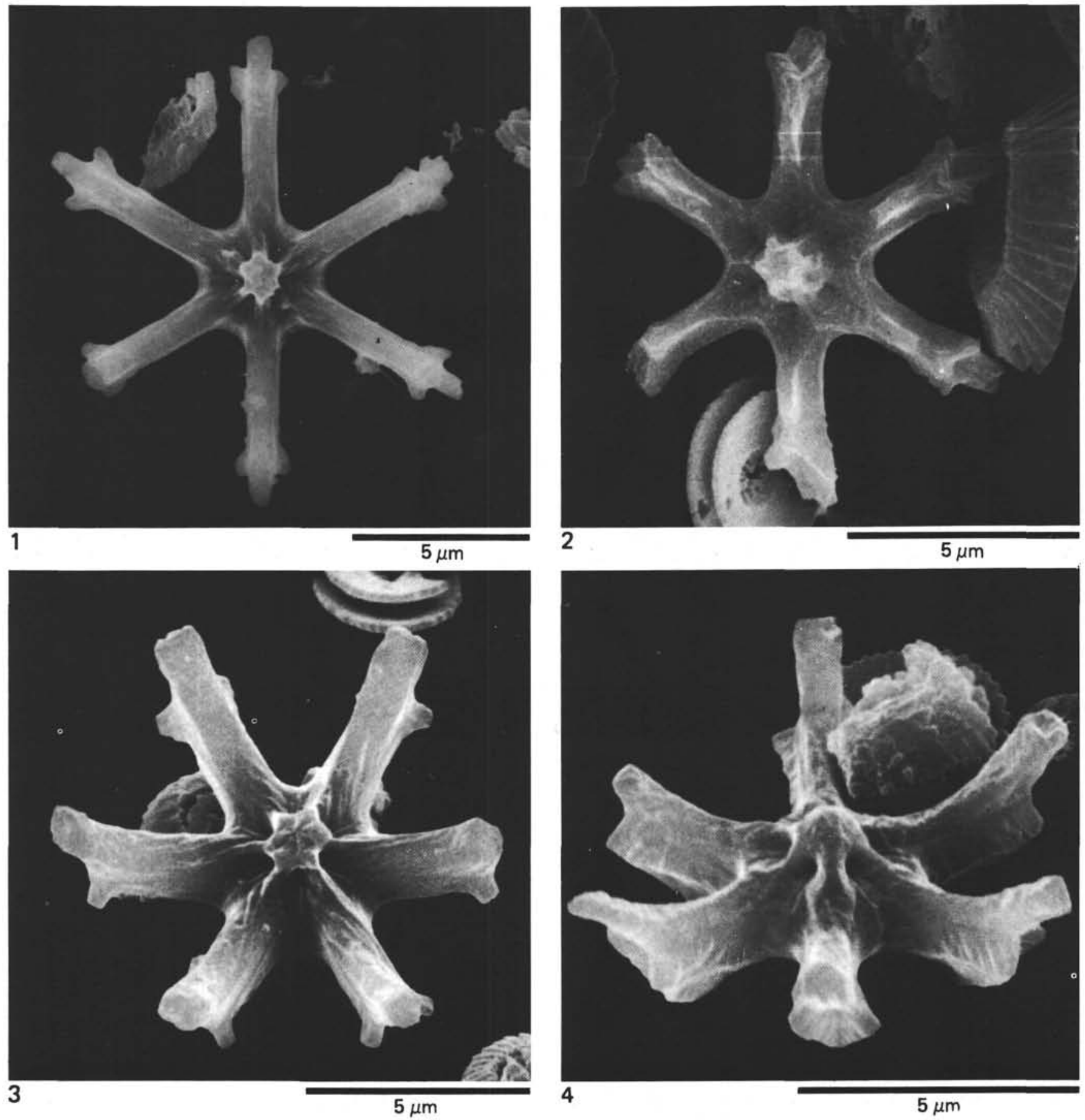

Plate 16. Proximal view, Discoaster surculus. 1. Sample 532-46,CC, SEM; 2. Distal view, Sample 532-55,CC, SEM; 3-4. Proximal view, Sample 532-55,CC, SEM. 

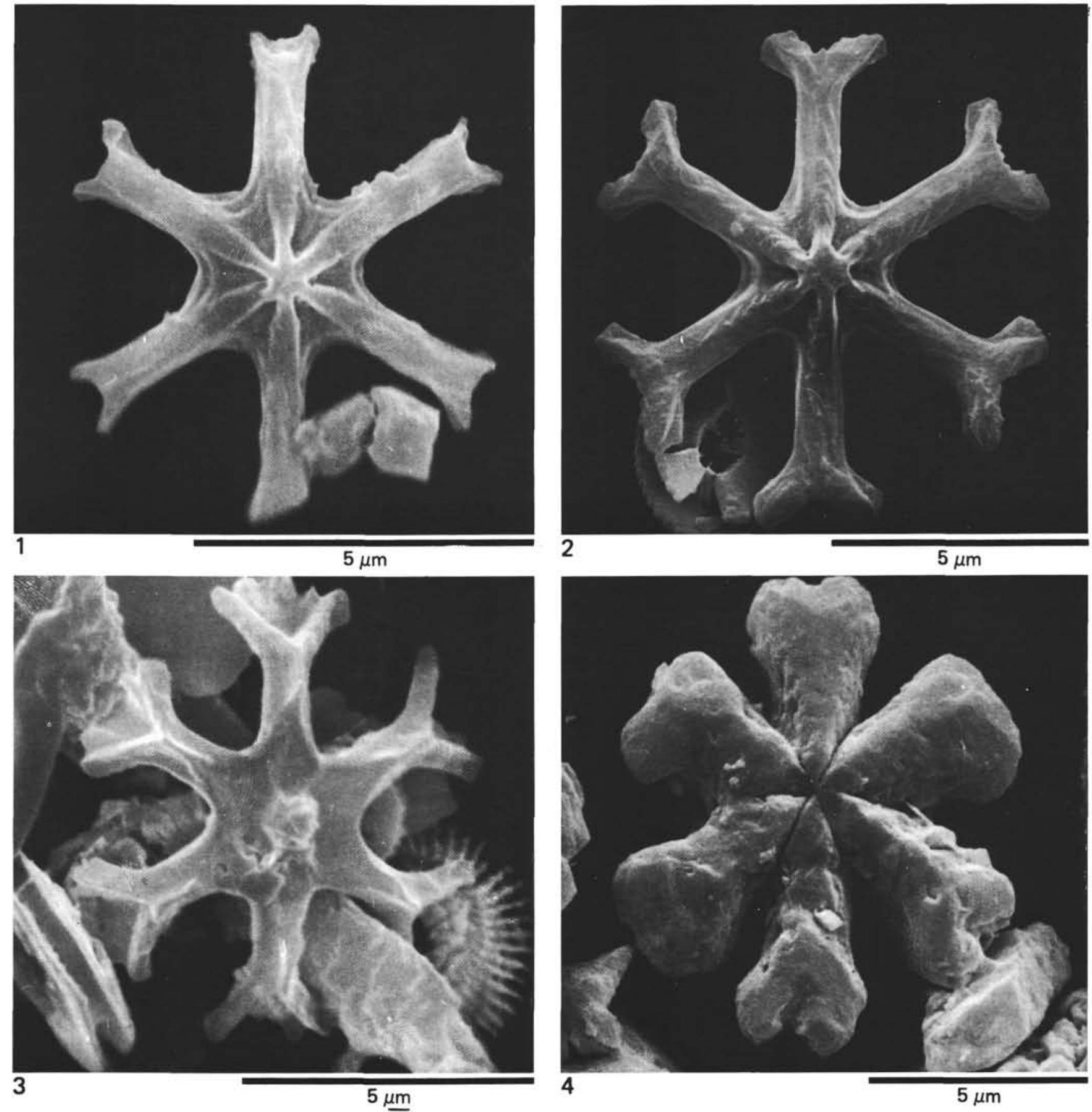

Plate 17. 1. Discoaster exilis, Sample $532-46, \mathrm{CC}$, SEM. 2. D. challengeri, Sample $532-55, \mathrm{CC}, \mathrm{SEM}$; 3. D. icarus, Sample $532-46, \mathrm{CC}$, SEM; 4. D. deflandrei, heavily calcified, Sample $530 \mathrm{~A}-37-1,96-97 \mathrm{~cm}$, SEM. 


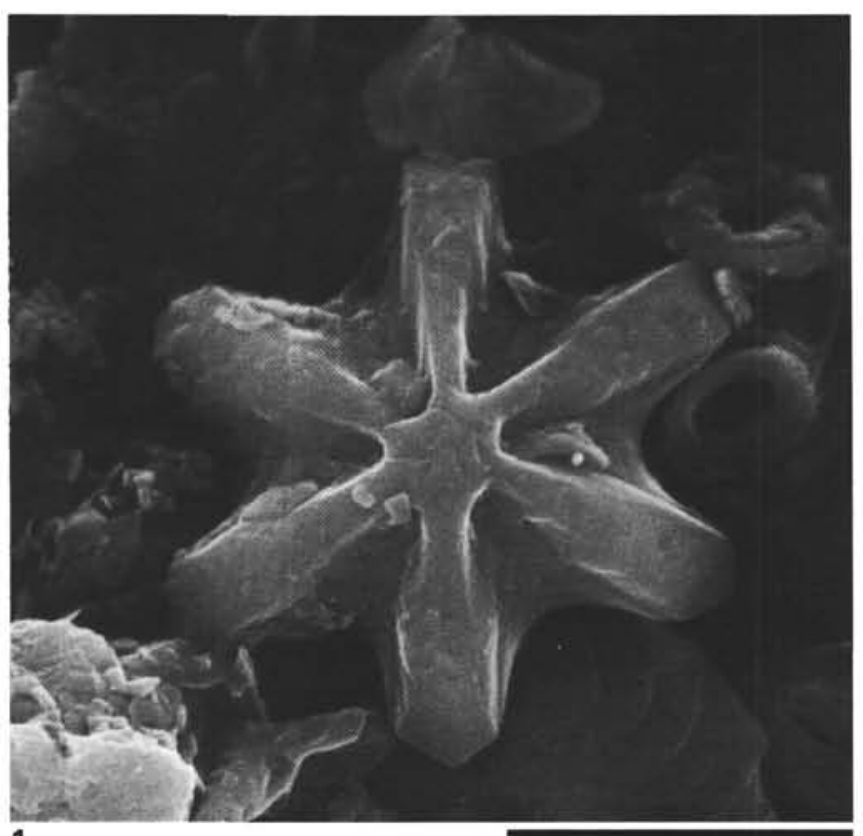

1

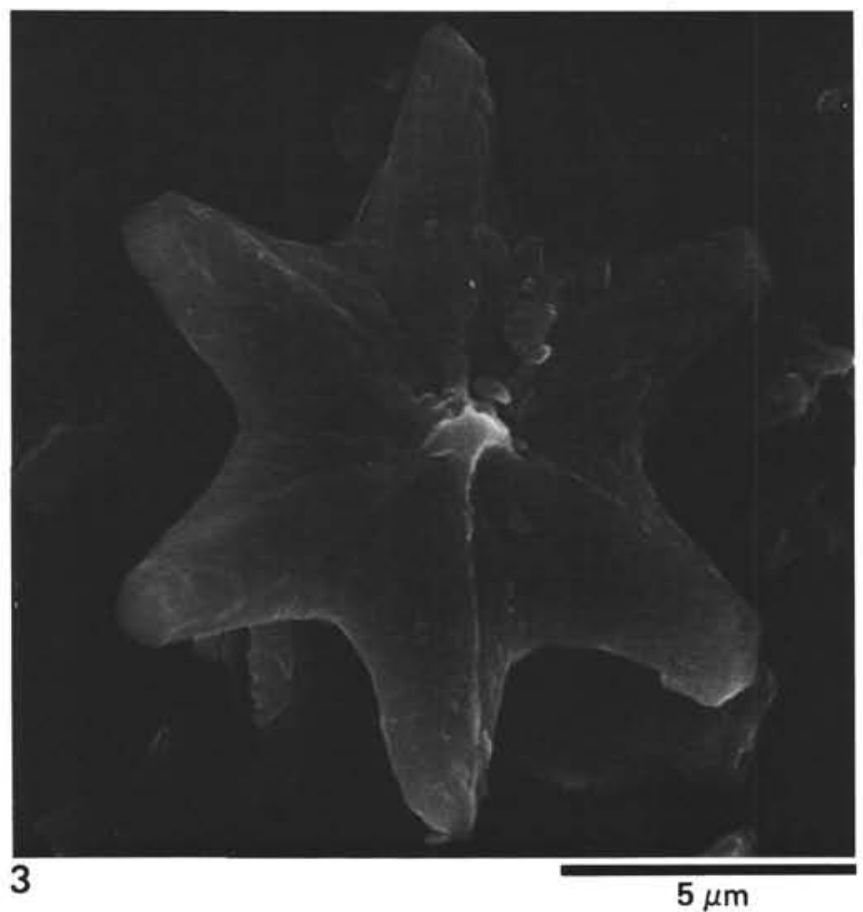

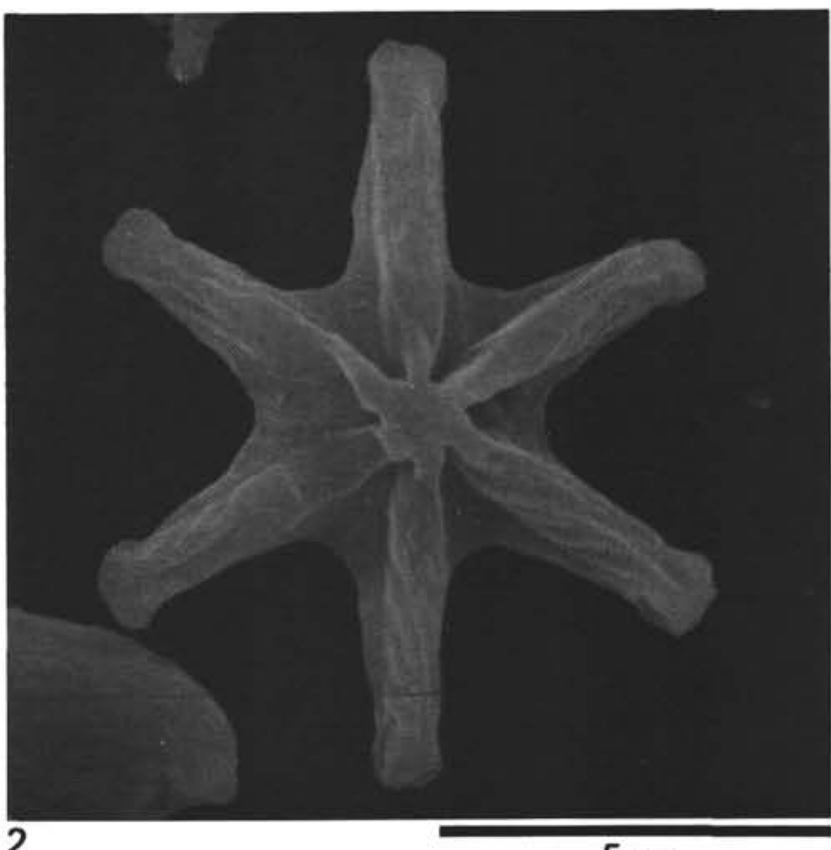

$5 \mu \mathrm{m}$

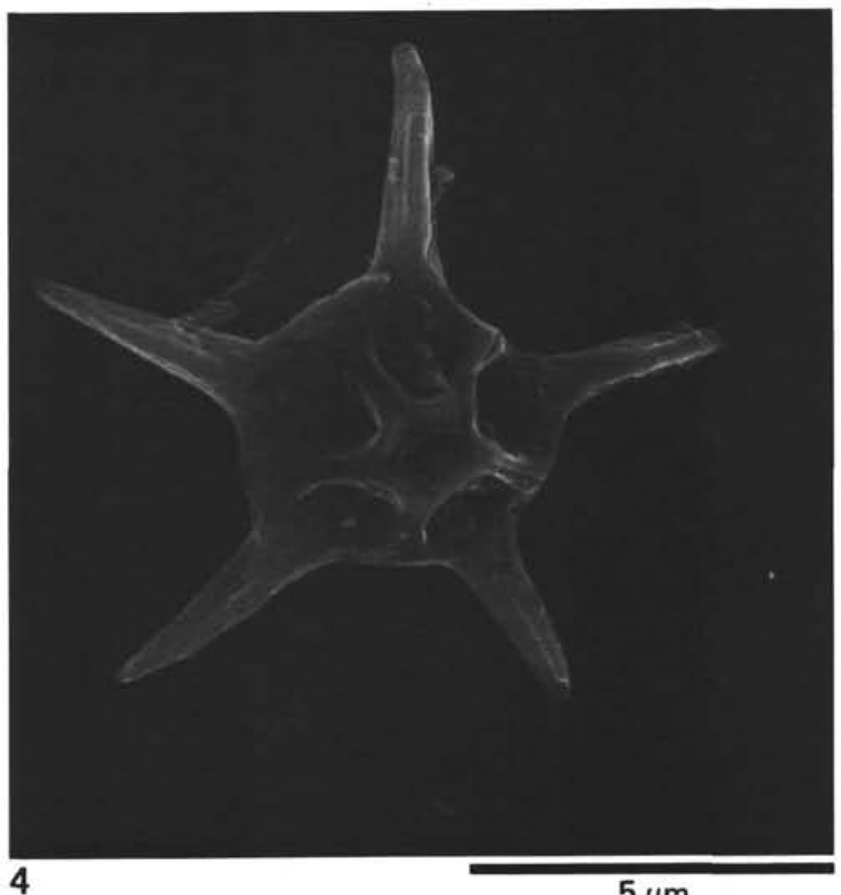

$5 \mu \mathrm{m}$

Plate 18. 1. Discoaster toralus, Sample $530 \mathrm{~A}-12-3,72-73 \mathrm{~cm}$, SEM*; 2. D. toralus, Sample 532-33,CC, SEM; 3. D. kugleri, Sample 530B$39, \mathrm{CC}, \mathrm{SEM}^{*} ;$ 4. $D$. berggrenii, Sample $530 \mathrm{~A}-12-3,72-73 \mathrm{~cm}, \mathrm{SEM}^{*}$. 

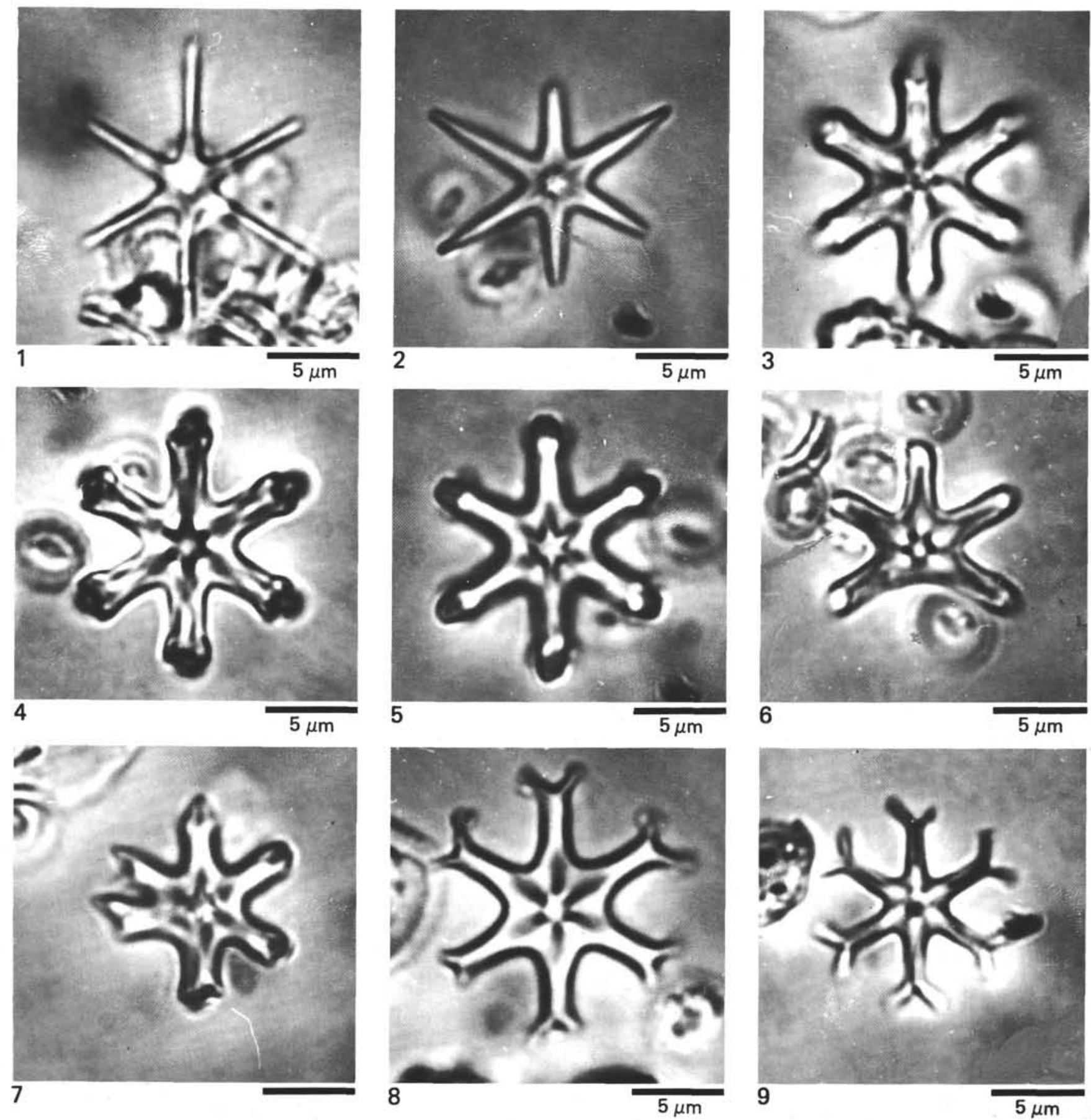

Plate 19. Sample 532-55,CC. 1-2. Discoaster brouweri, NL; 3-7. D. surculus, NL; 8. Discoaster variabilis, NL; 9. D. loeblichii, CC, NL. 

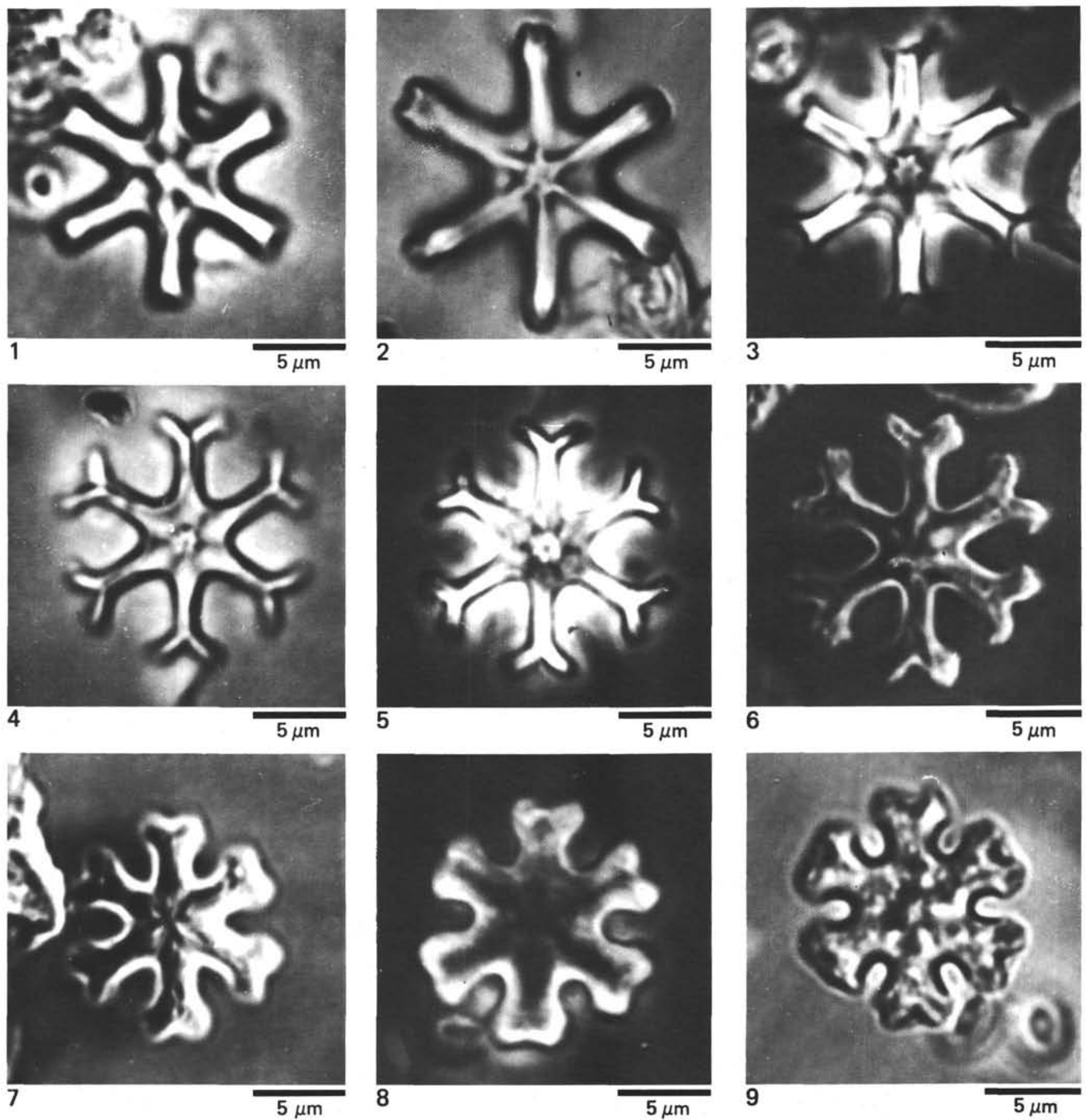

Plate 20. 1-2. Discoaster cf. D. surculus, NL; 3. D. exilis, PHC; 4. D. variabilis, NL; 5. D. variabilis, PHC; Sample 532-55,CC. 6. D. variabilis, NL (Al); 7-8. D. pansus, NL (Al); 9. D. pansus, NL. Sample 532-33,CC. 

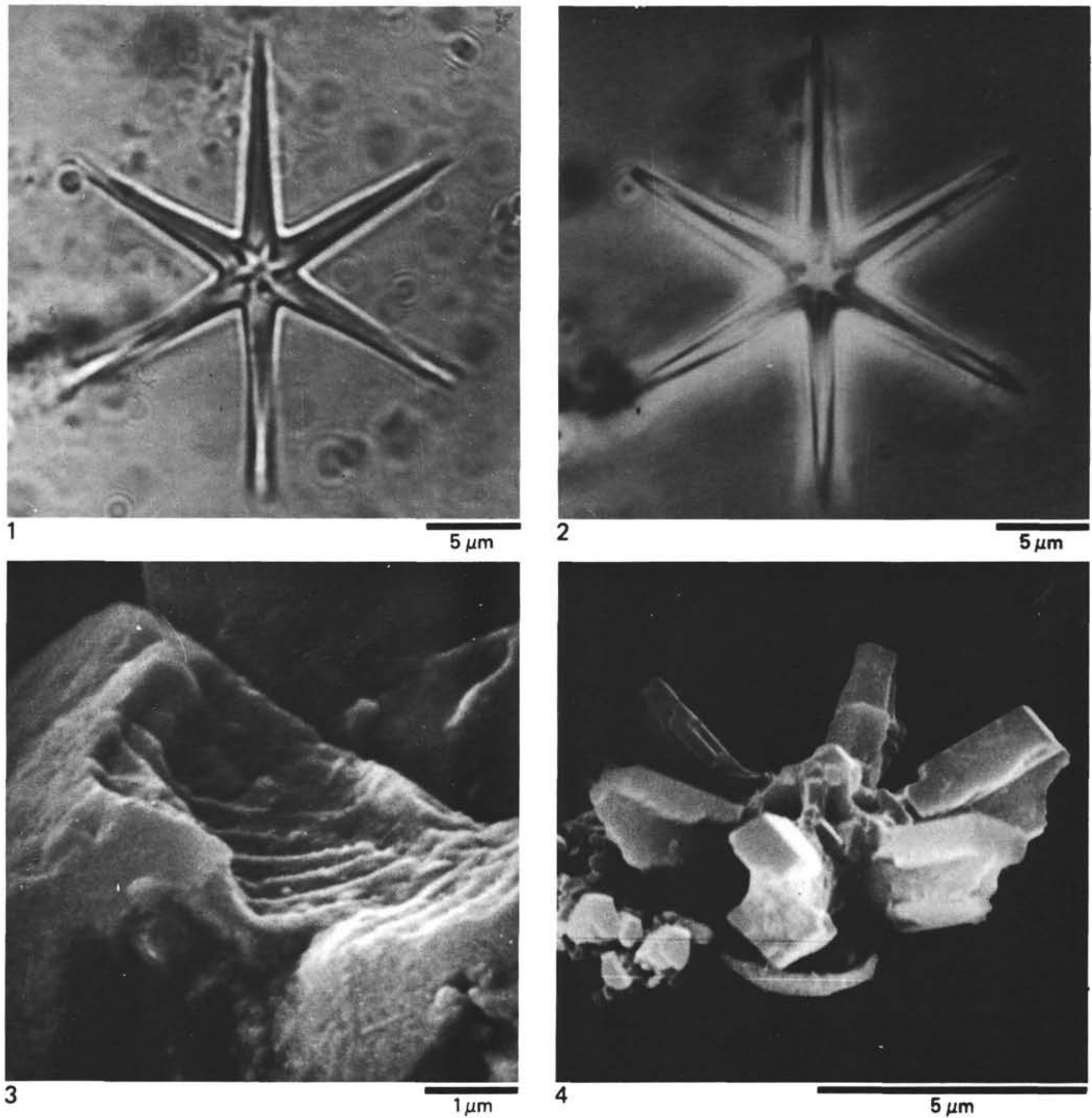

Plate 21. 1. Discoaster brouweri, Sample 530A-15-5, 77-78 cm, NL; 2. D. brouweri, Sample 530A-15-5, 77-78 cm, PHC; 3. Braarudosphaera bigelowi, corrosion etching, detail of Plate 25, Figure 2, Sample 530A-37-1, 96-97 cm, SEM; 4. Discoaster sp., heavily calcified, Sample $532-55$, CC, SEM. 

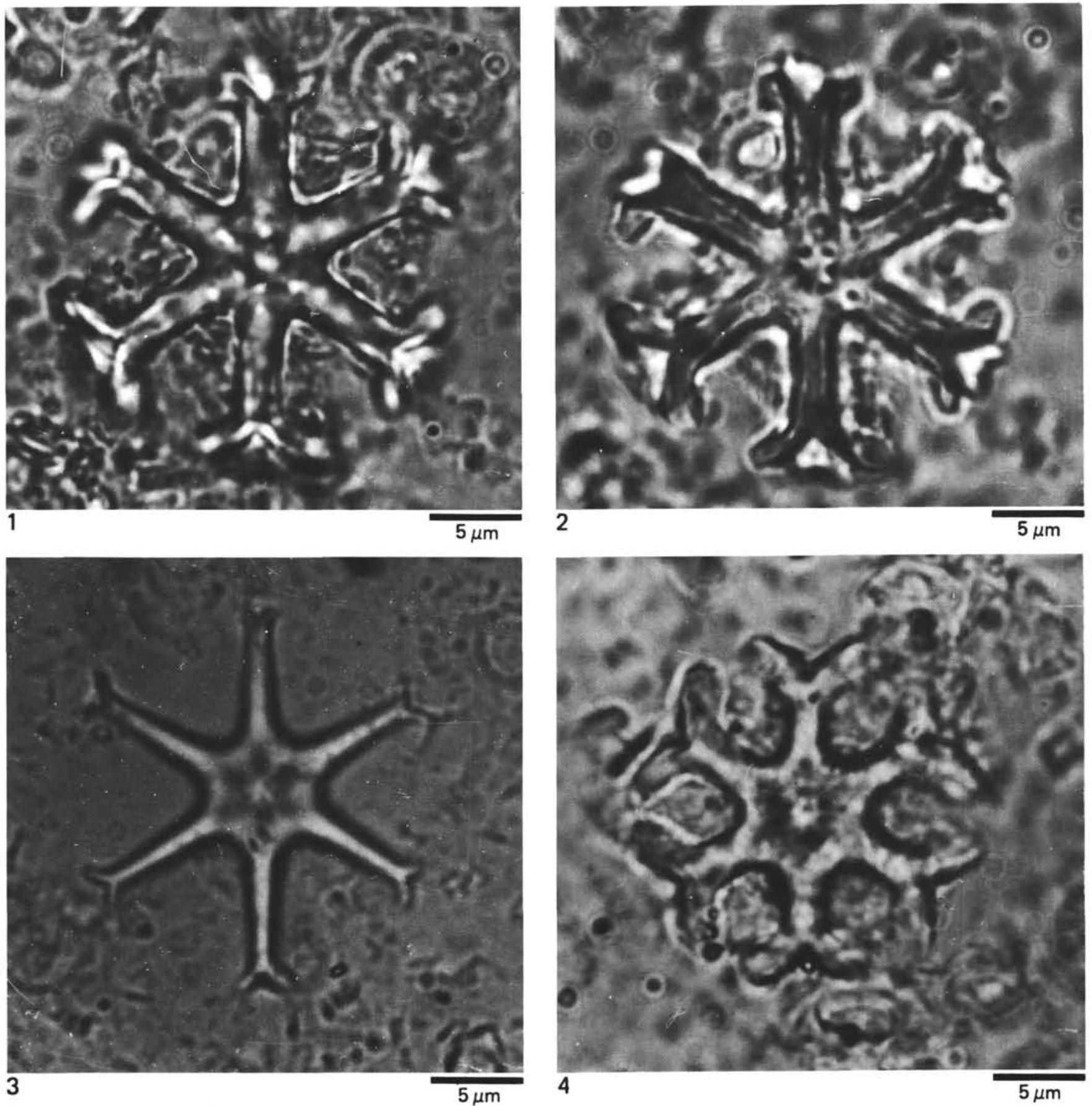

Plate 22. 1. Discoaster pseudovariabilis, Sample 530A-15-6, 77-78 cm, NL, high focus; 2. D. pseudovariabilis, Sample 530A-15-6, 77-78 cm, NL, low focus; 3. D. decorus, Sample $530 \mathrm{~A}-12-3,72-73 \mathrm{~cm}$, NL; 4. D. icarus, Sample $530 \mathrm{~A}-15-6,77-78 \mathrm{~cm}$, NL. 

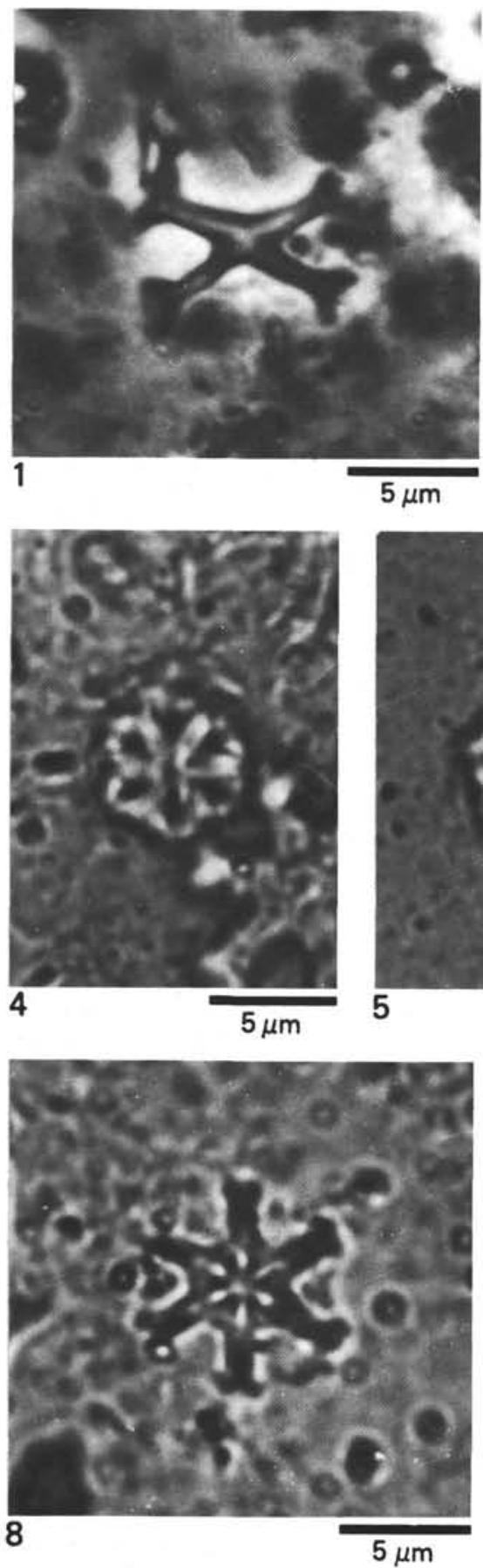
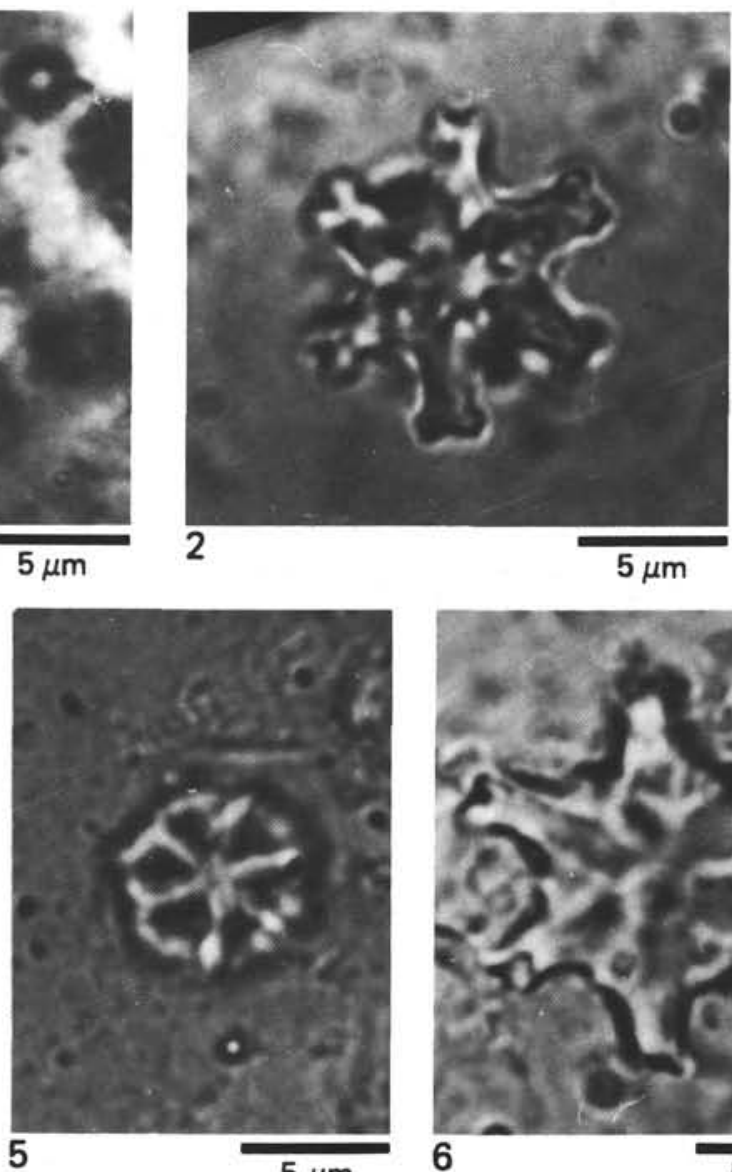

$5 \mu \mathrm{m}$

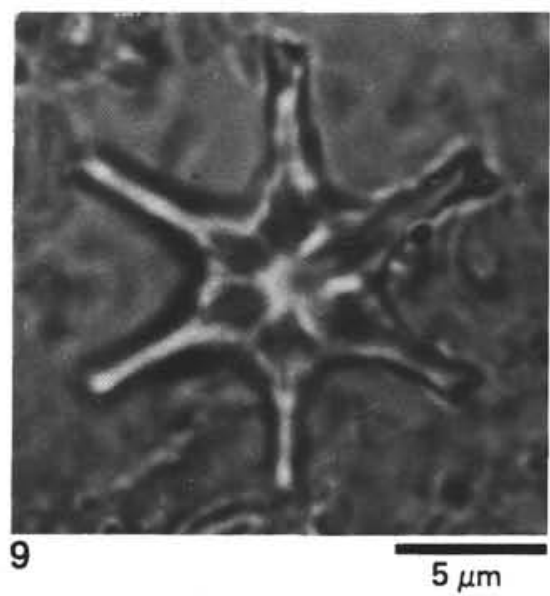

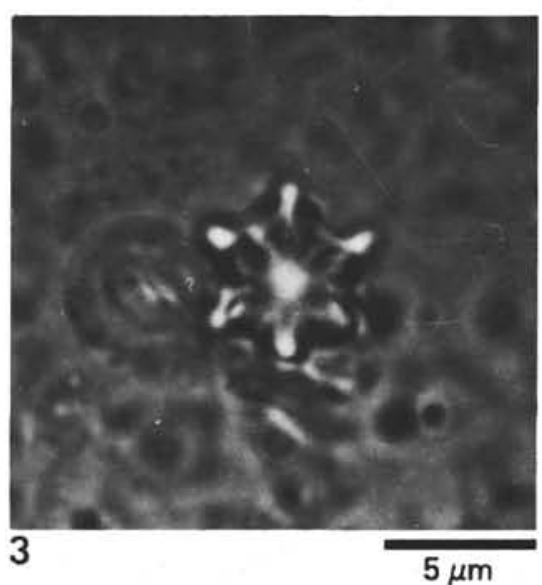
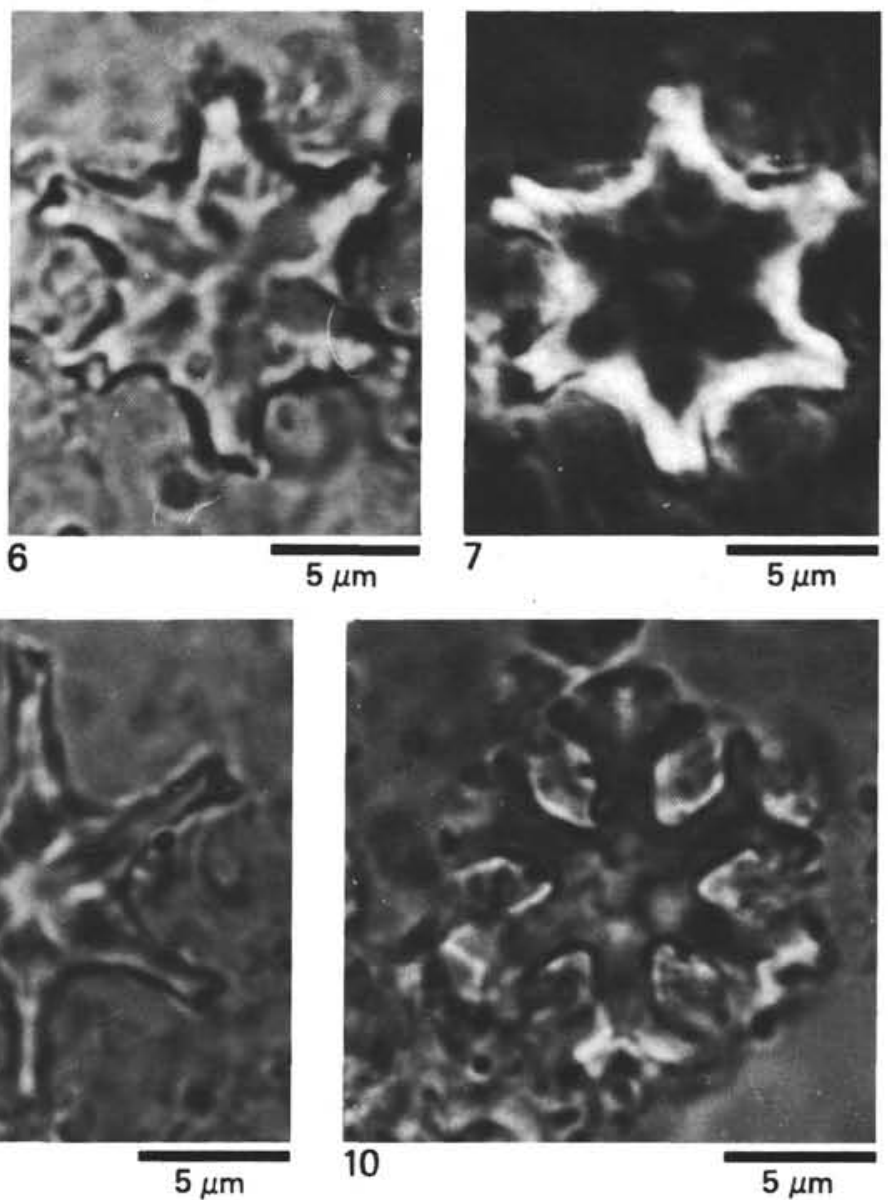

Plate 23. 1. Discoaster variabilis, Sample 530A-20-4, 70-71 cm, NL; 2-3. D. musicus, Sample 530A-20-4, 70-71 cm, NL; 4-5. Catinaster coalitus, Sample 530A-18-3, 73-74 cm, NL; 6. D. kugleri, Sample 530A-15-6, 77-78 cm, NL; 7. D. kugleri, Sample 530A-15-6, 77-78 cm, PHC; 8. D. bollii, Sample 530A-20-4, 70-71 cm, NL; 9. D. exilis, Sample 530A-20-4, 70-71 cm, NL; 10. D. icarus, Sample 530A-15-6, $77-78 \mathrm{~cm}$, NL. 

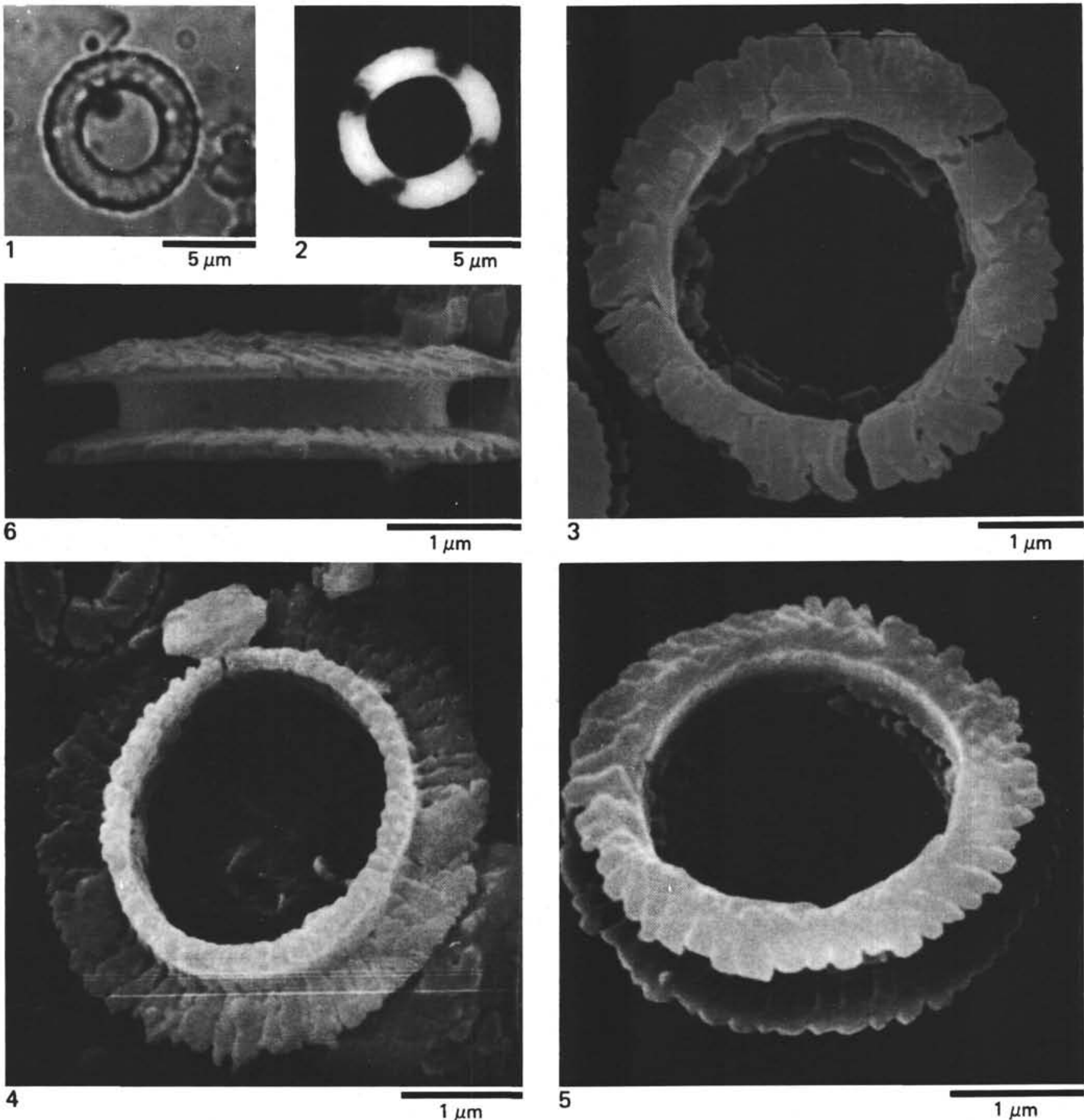

Plate 24. Calcidiscus aequiscutum. 1. Sample 530A-24-2, 28-29 cm, NL; 2. Sample 530A-24-2, 28-29 cm, XN; 3-4. Sample 532-36,CC, SEM; 5. Sample 532-55,CC, SEM; 6. Sample 532-36,CC, side view, SEM. 

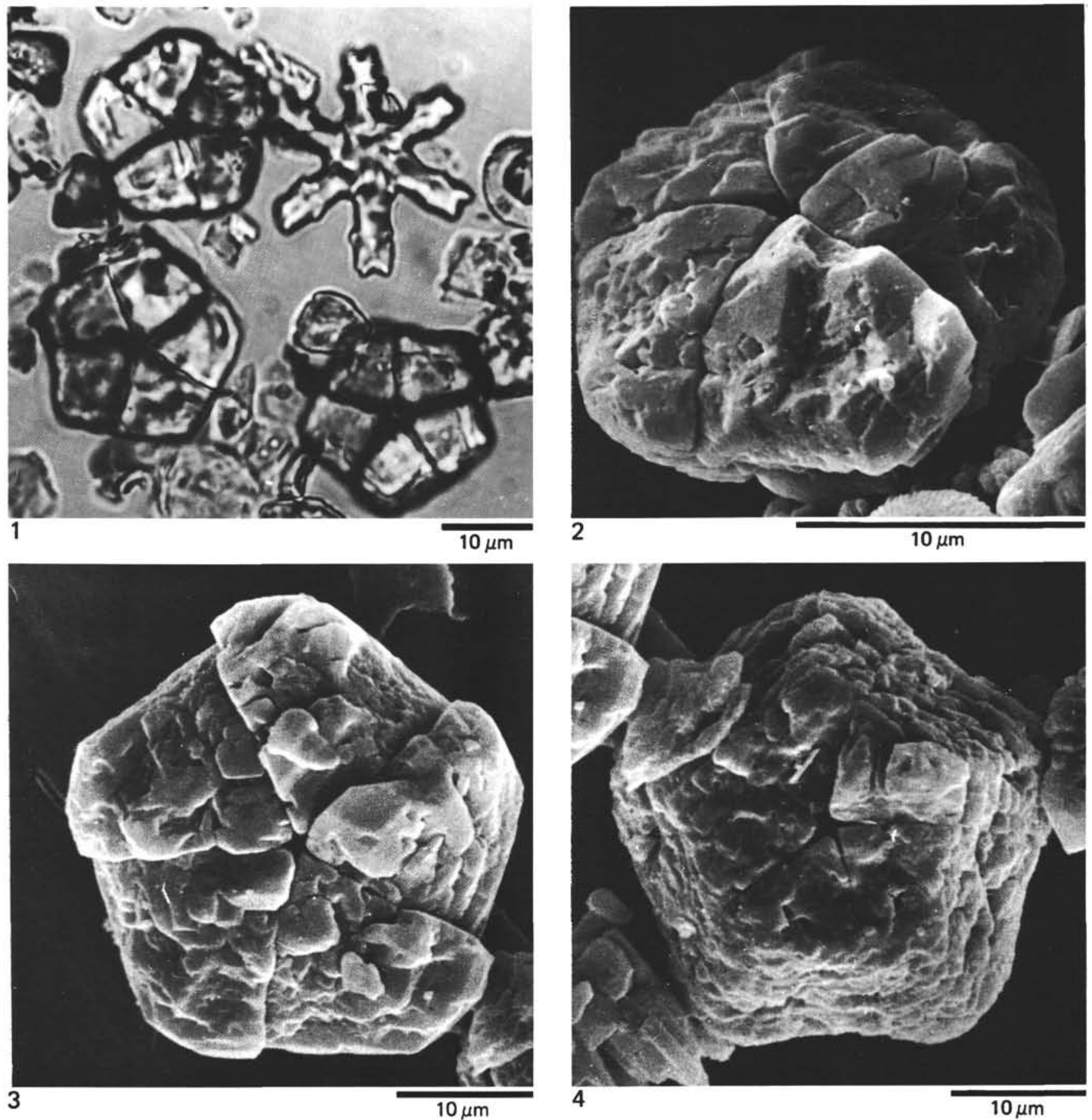

Plate 25. Sample 530A-37-1, 96-97 cm. 1. Braarudosphaera bigelowi and Discoaster tanii nodifer, NL; 2-3. B. bigelowi, distal view, SEM; 4. B. bigelowi, proximal view, SEM. 


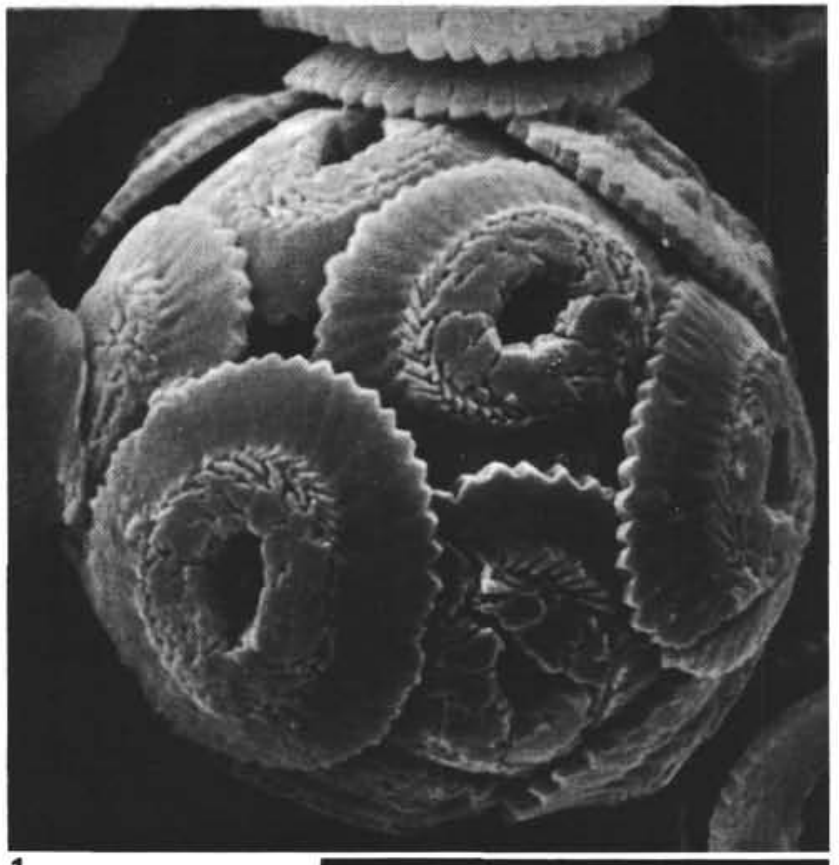

1

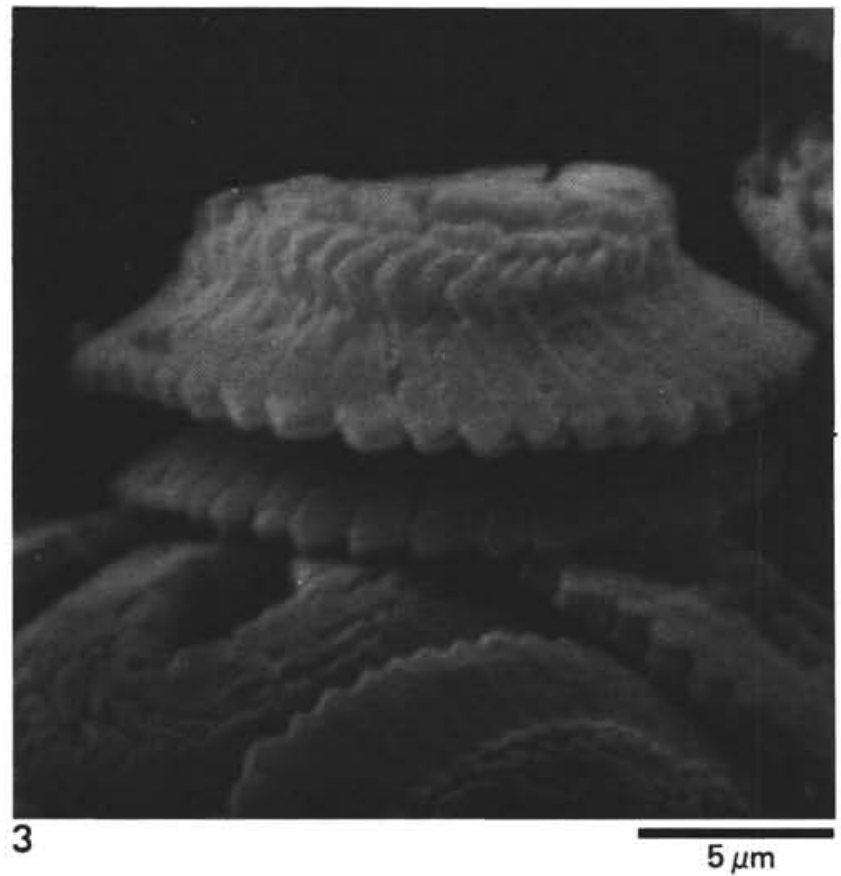

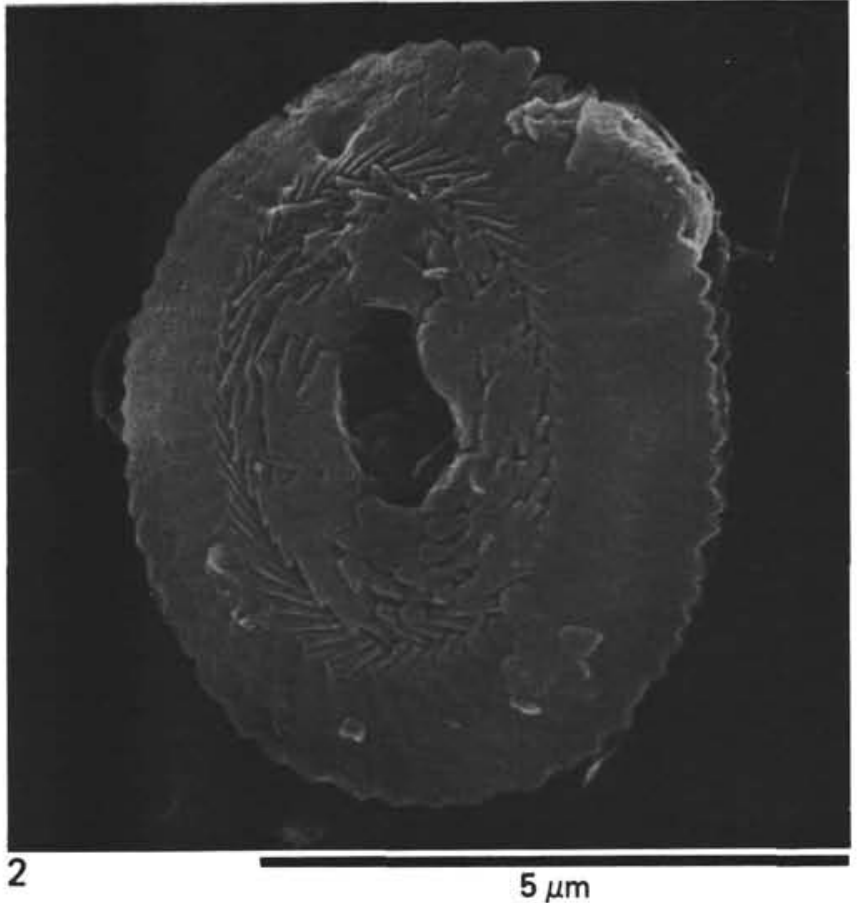

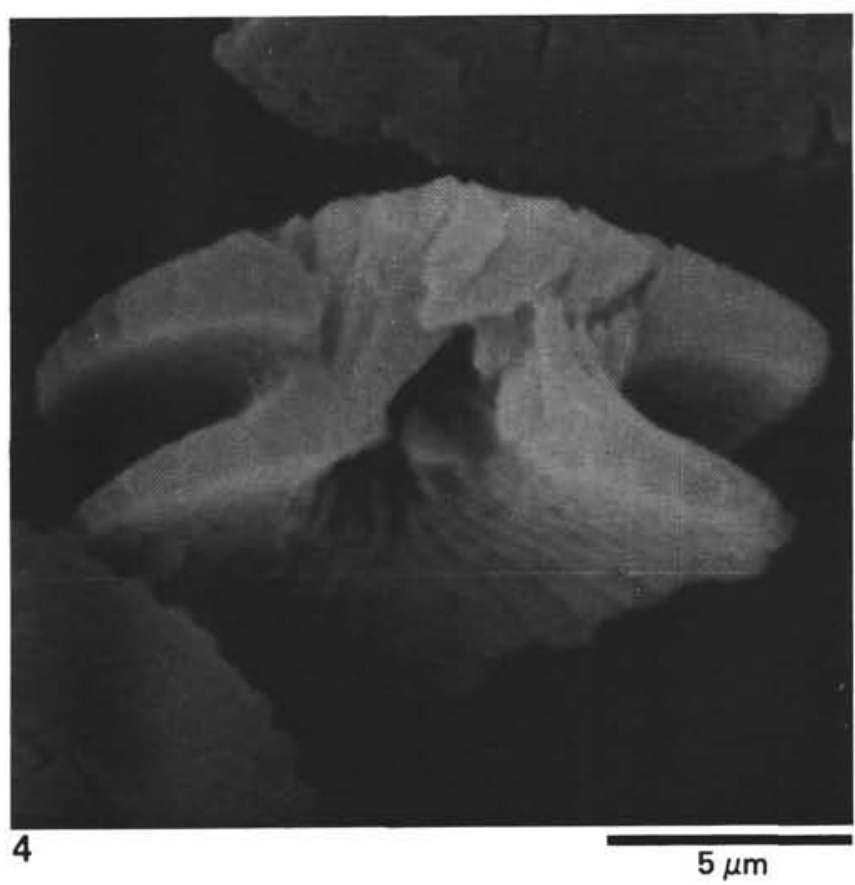

Plate 26. Reticulofenestra pseudoumbilica. 1. Coccosphere, Sample 532-55,CC, SEM; 2. Distal view, Sample 530B-29,CC, SEM*; 3. Side view, Sample 532-55,CC, SEM; 4. Fractured cross section, Sample 532-55,CC, SEM. 

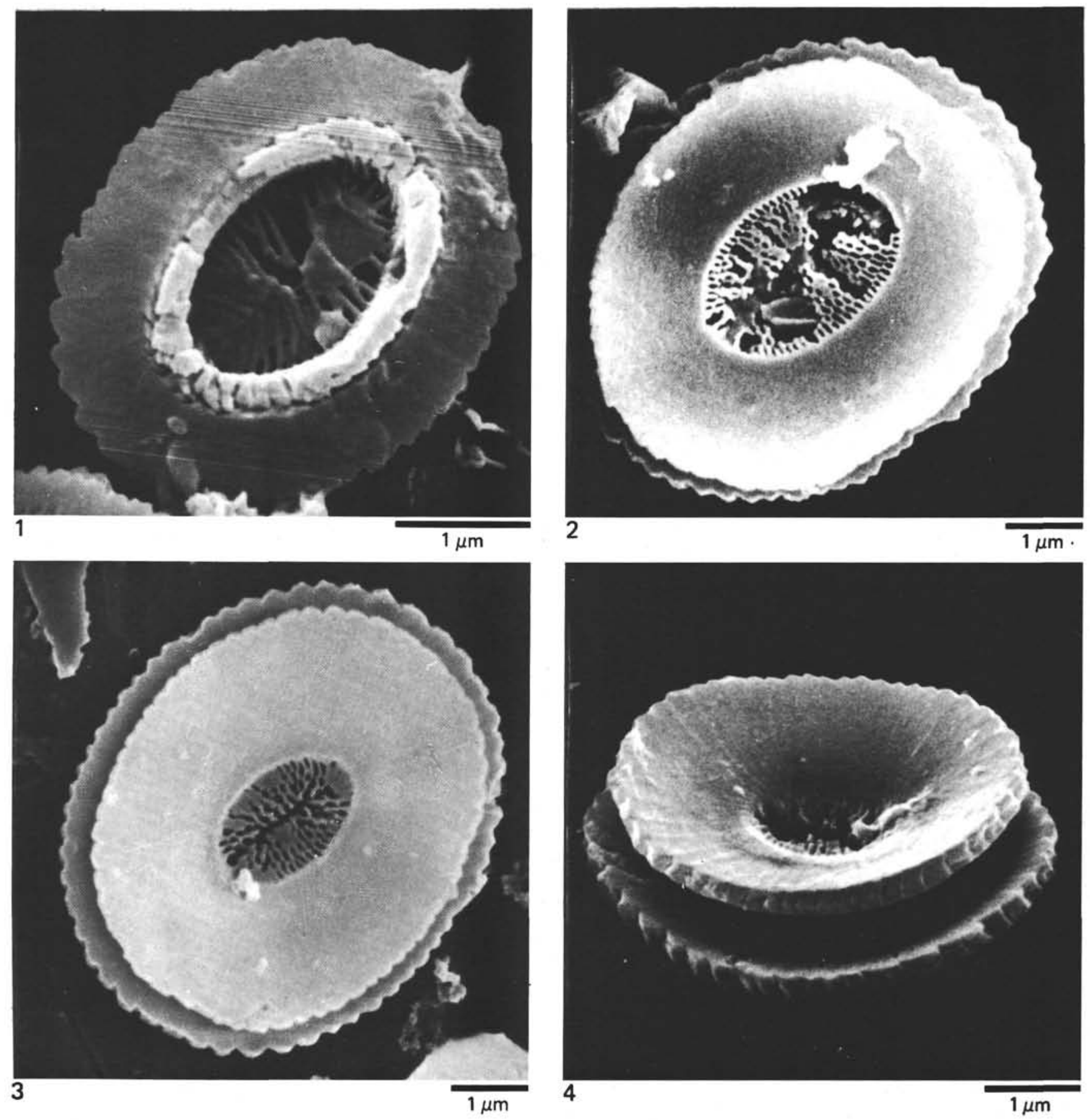

Plate 27. Reticulofenestra pseudoumbilica. 1. Distal view of proximal plate, Sample 532-36,CC, SEM; 2. Proximal view, Sample 532-55,CC, SEM; 3. Proximal view, Sample 532-46,CC, SEM; 4. Oblique proximal view, Sample 532-46,CC, SEM. 


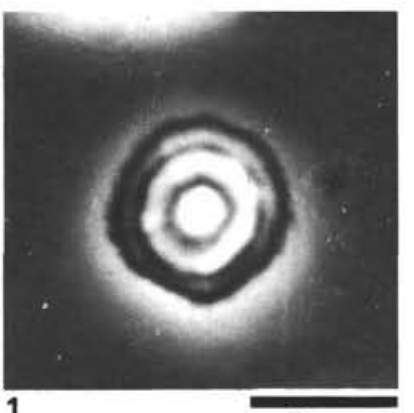

1

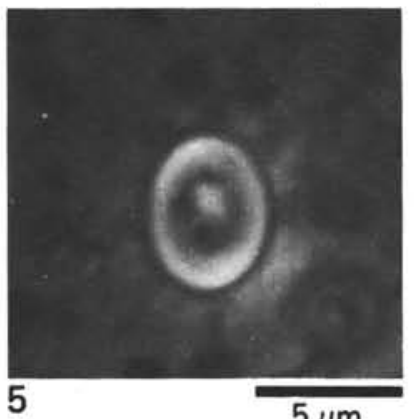

$5 \mu \mathrm{m}$

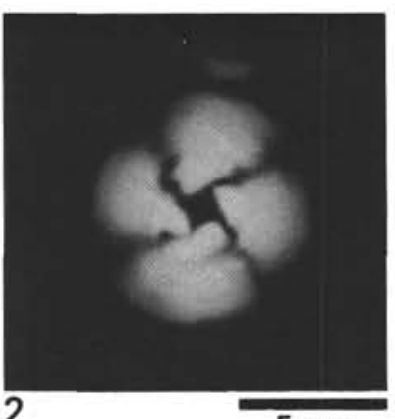

$5 \mu \mathrm{m}$

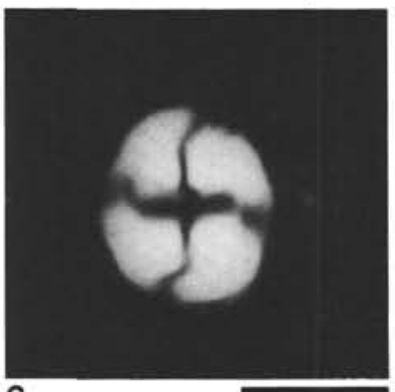

$5 \mu \mathrm{m}$

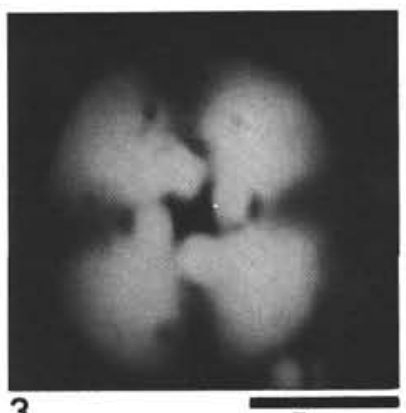

$5 \mu \mathrm{m}$

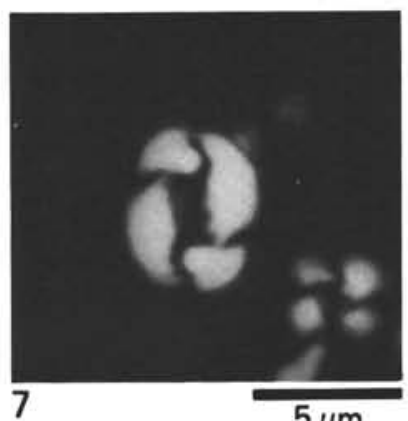

$5 \mu \mathrm{m}$

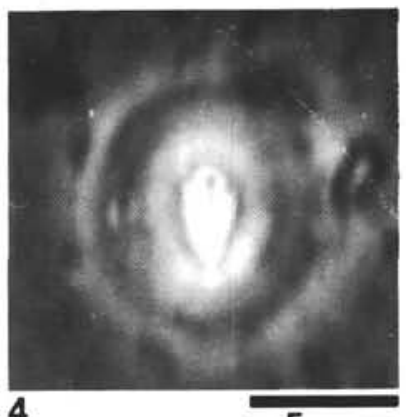

$5 \mu \mathrm{m}$

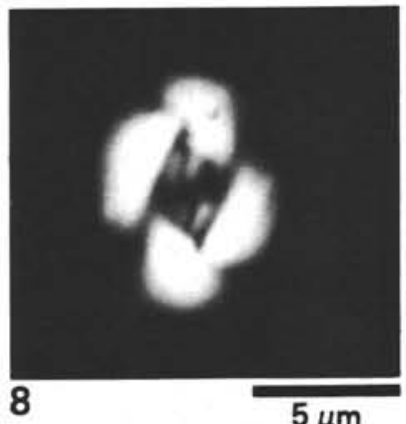

$5 \mu \mathrm{m}$
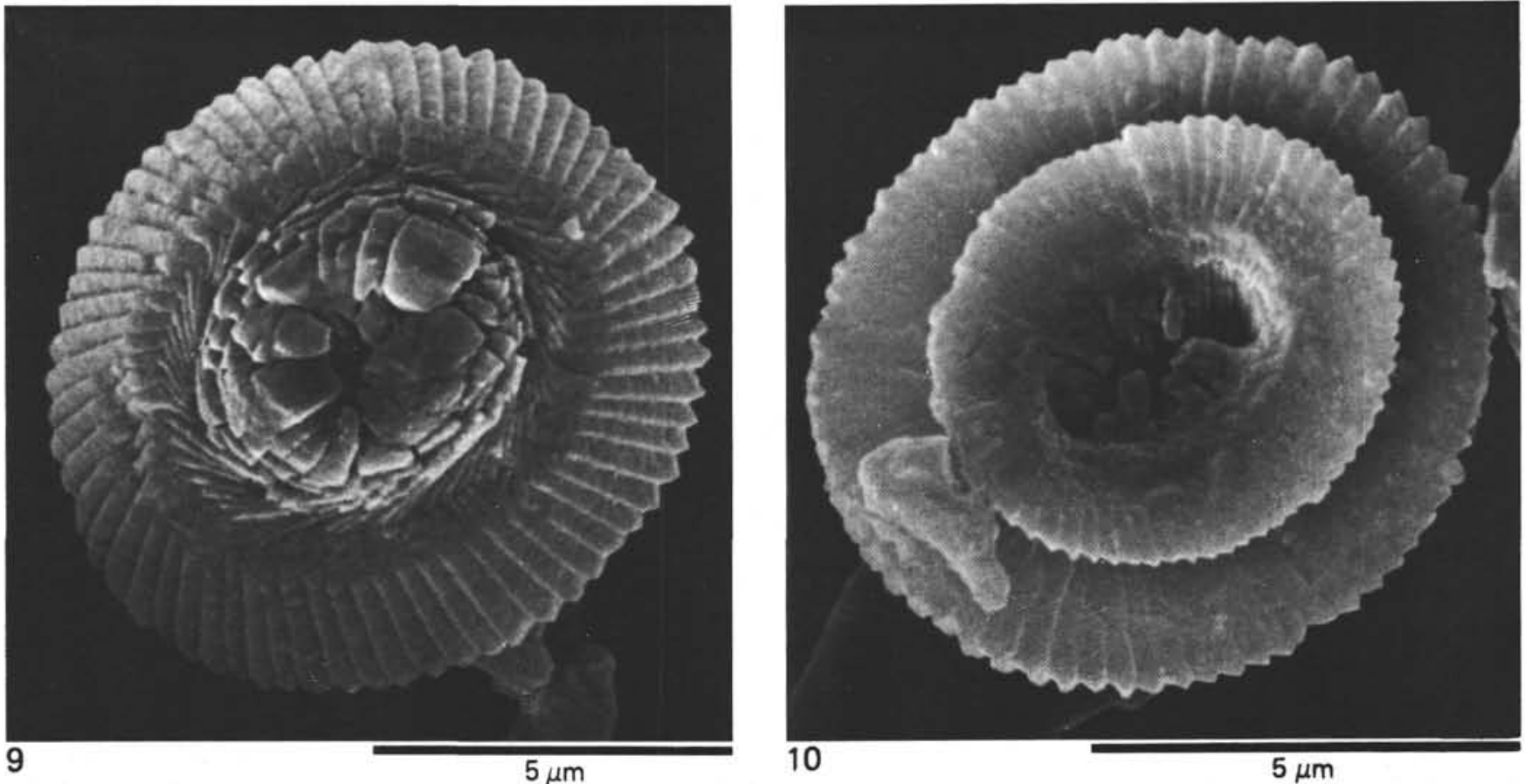

$5 \mu \mathrm{m}$

10

$5 \mu \mathrm{m}$

Plate 28. 1. Cyclicargolithus floridanus, Sample 530A-49-1, 23-24 cm, PHC; 2. C. floridanus, Sample 530A-35-1, 30-31 cm, XN; 3. C. abisectus, Sample 530A-35-1, 30-31 cm, XN; 4, 5. C. orangensis, Sample 530A-35-1, 30-31 cm, XN; 6. Reticulofenestra gartneri, Sample $530 \mathrm{~A}-35-1,30-31 \mathrm{~cm}$, PHC; 7. Coccolithus orangensis, Sample 530A-35-1, 30-31 cm, PHC; 8. $R$. gartneri, Sample 530A-35-1, 30-31 cm, $\mathrm{XN}$; 9. $R$. bisecta, distal view, Sample 530A-37-1, 96-97 cm, SEM; 10. $R$. bisecta, proximal view, Sample 530A-37-1, 96-97 cm, SEM. 

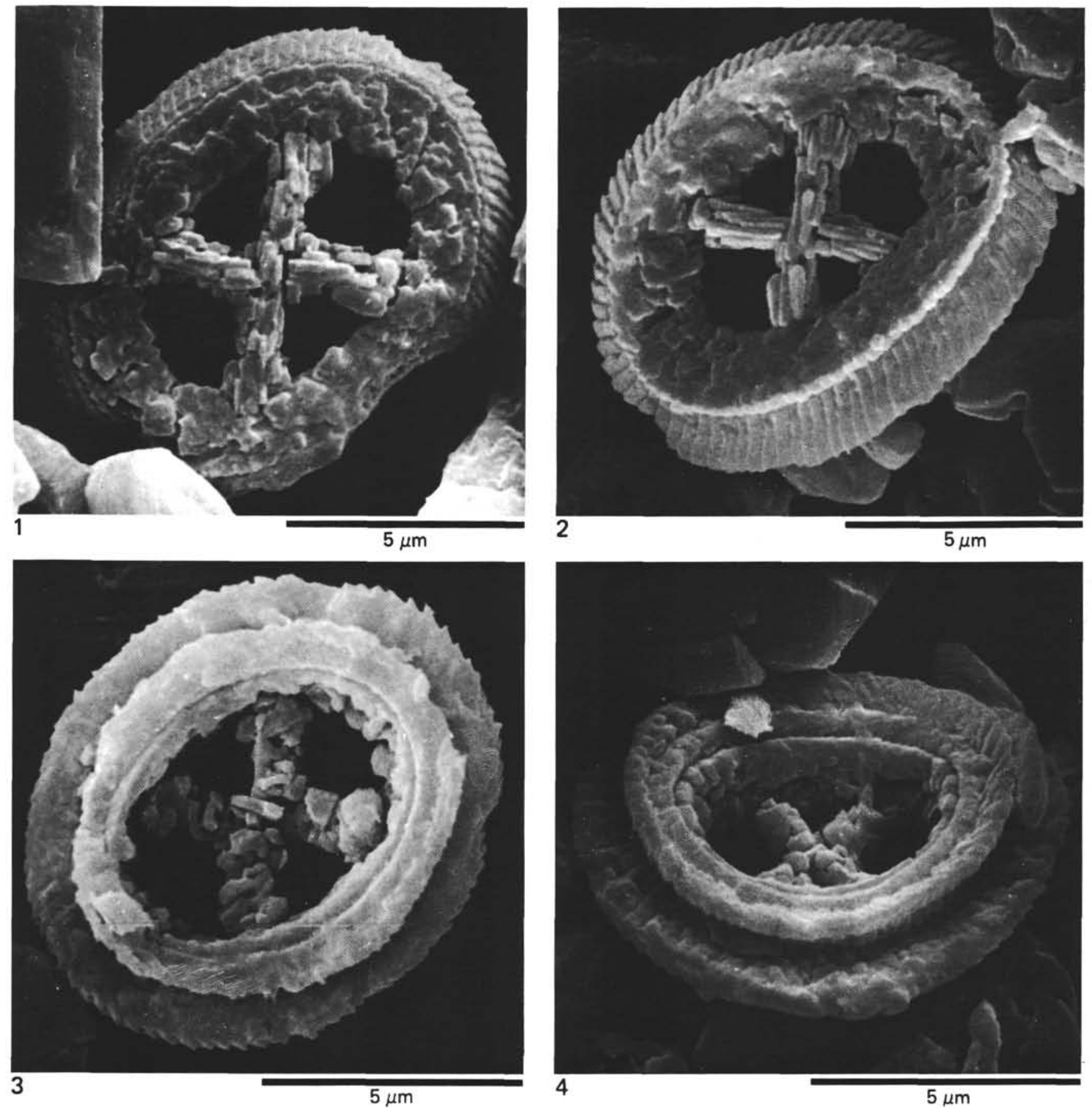

Plate 29. Chiasmolithus altus, Sample 530A-37-1, 96-97 cm, SEM. 1-2. Distal view. 3. Proximal view. 4. Oblique proximal view. 

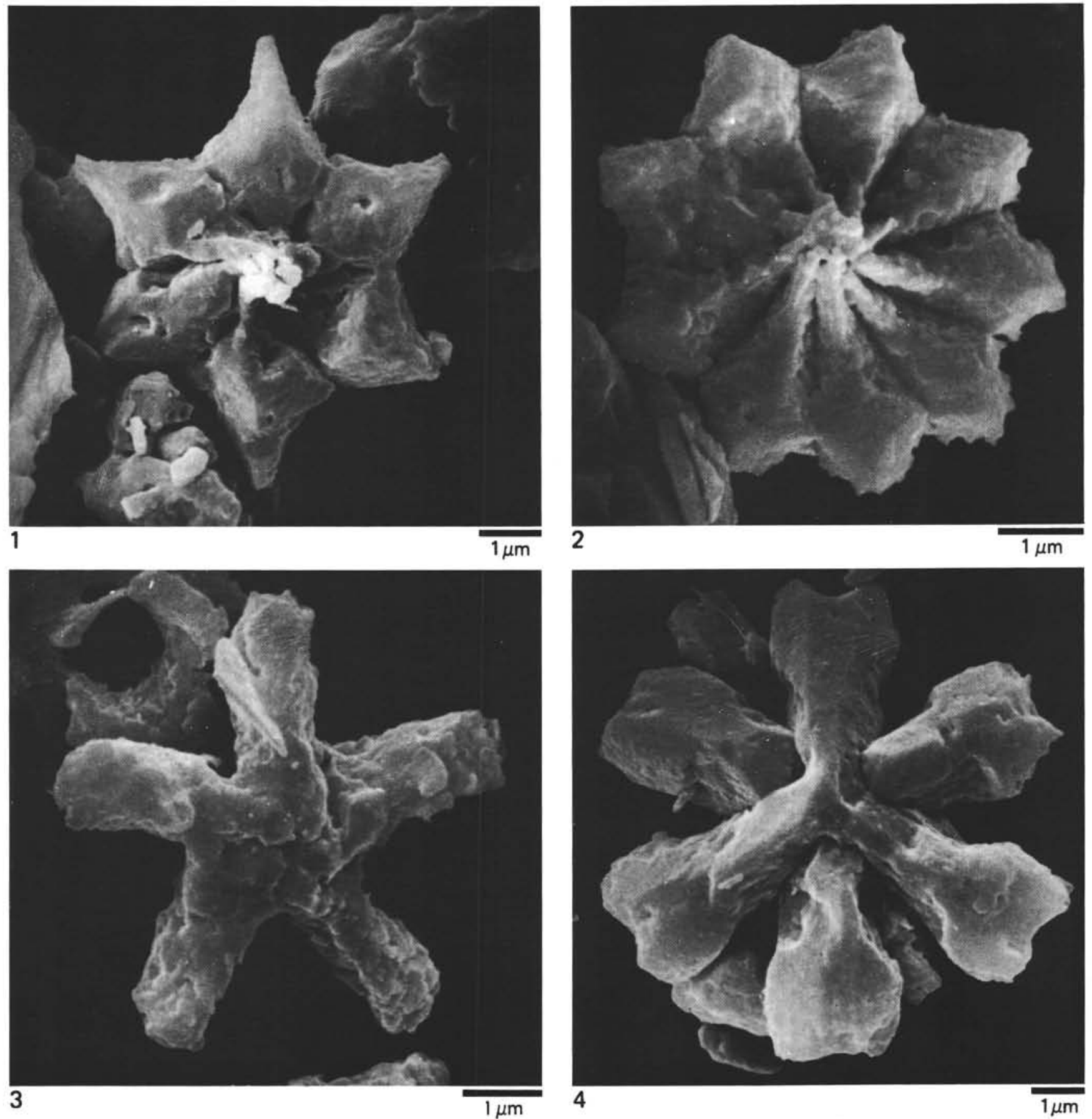

Plate 30. Sample 530A-37-1, 96-97 cm. 1. Discoaster saipanensis, SEM; 2. D. barbadiensis, SEM; 3. D. tanii,SEM; 4. D. trinus, heavily calcified, SEM. 

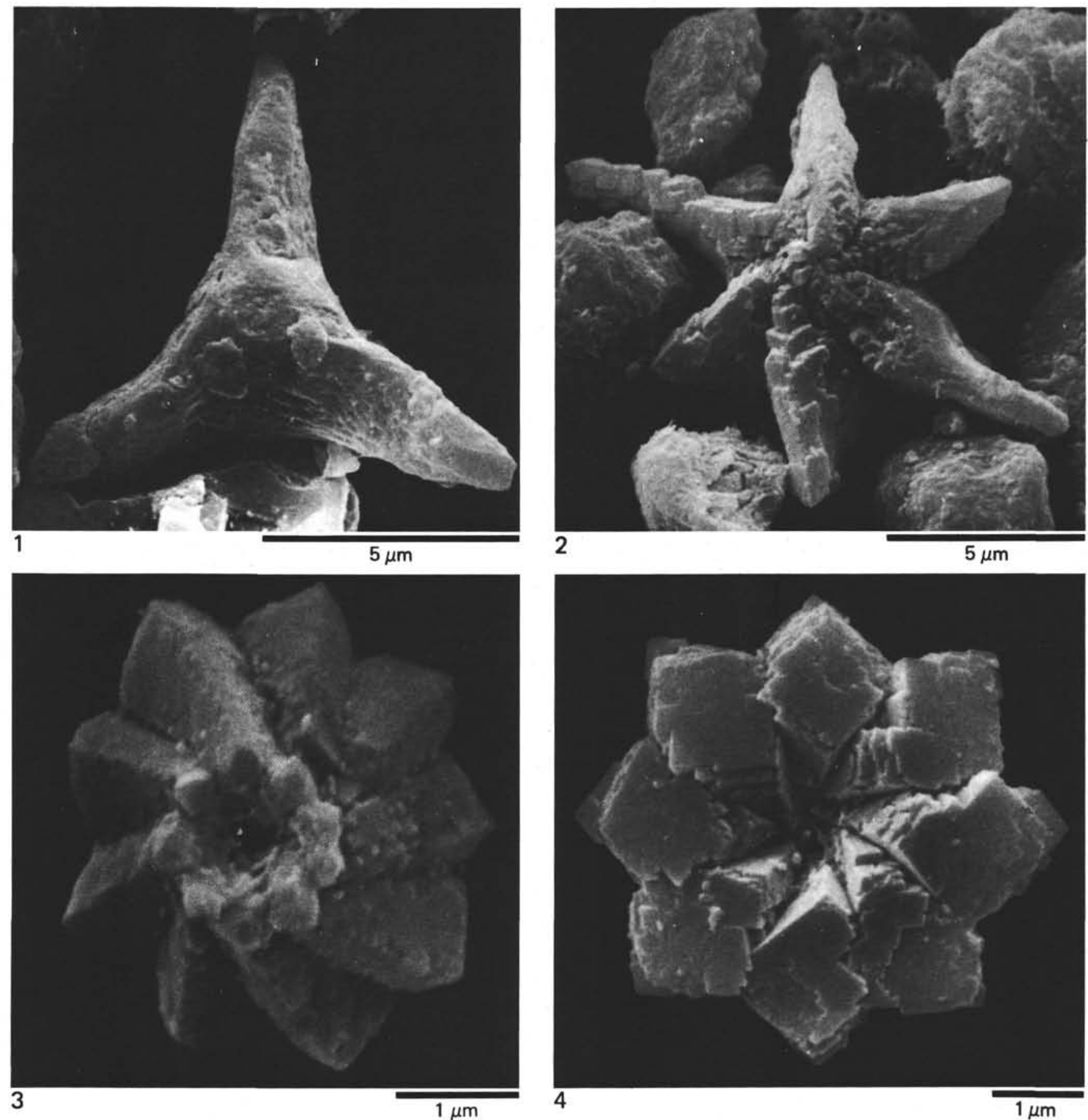

Plate 31. Sample 530A-39-2,21-22 cm. 1. Tribrachiatus orthostylus, SEM; 2. Discoaster lodoensis, SEM; 3. Discoasteroides kuepperi, proximal view, heavily calcified, SEM; 4. D. kuepperi, distal view, heavily calcified, SEM. 

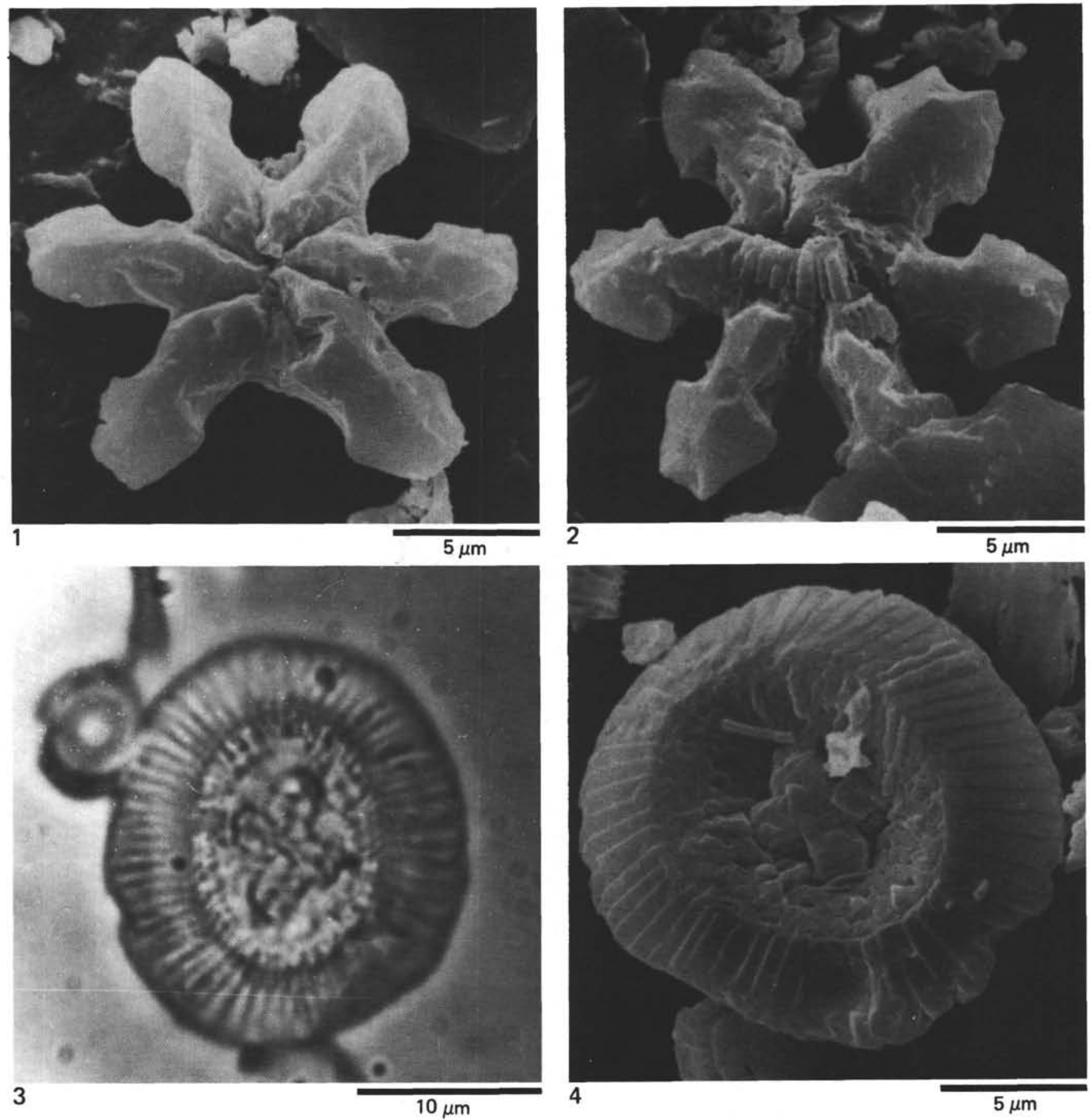

Plate 32. Sample 530A-43-2, 20-21 cm. 1-2. Discoaster binodosus, SEM; 3. Chiasmolithus consuetus, NL; 4. C. consuetus, distal view, SEM. 

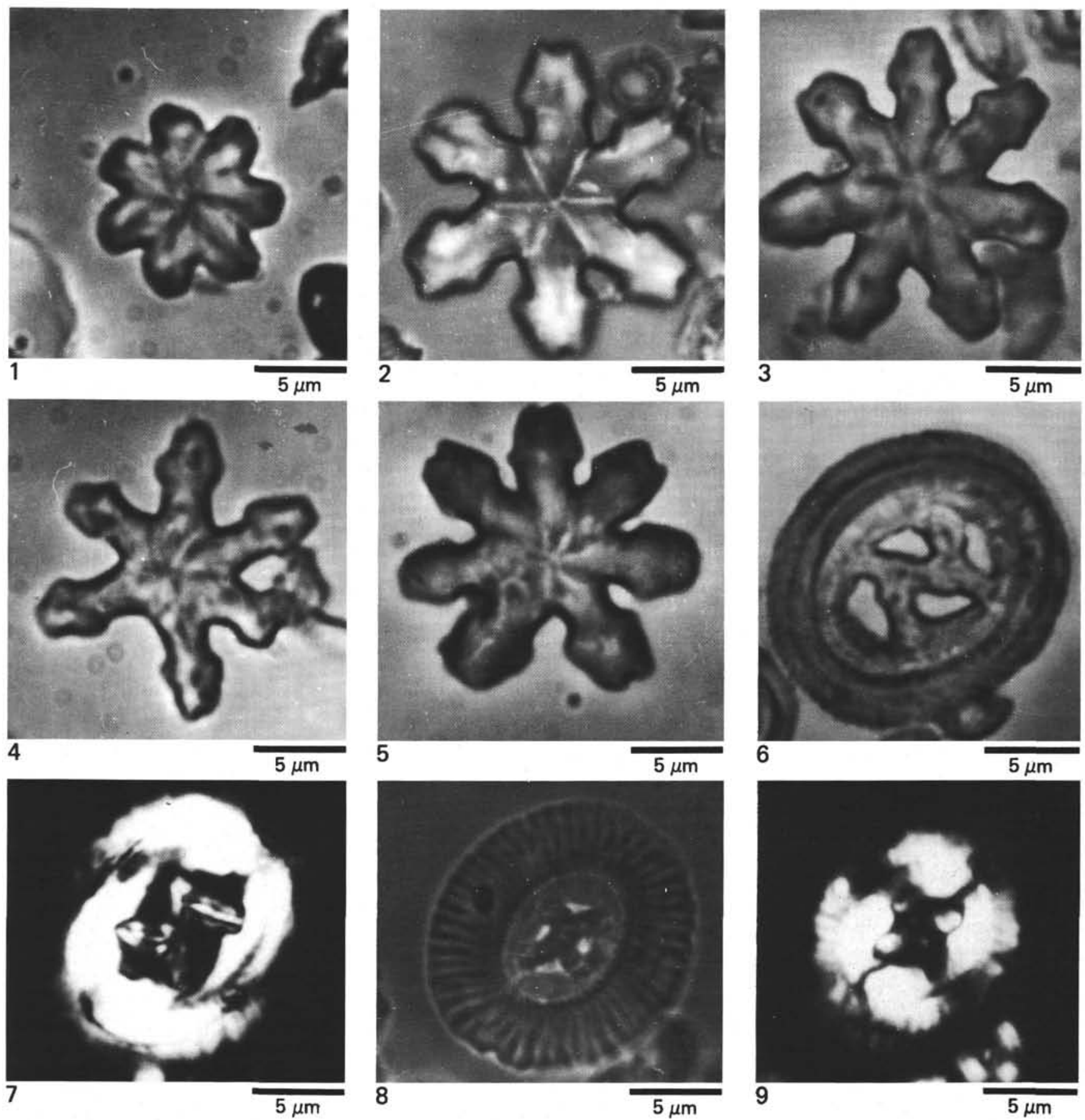

Plate 33. Sample 530A-43-2, 20-21 cm. 1. Discoaster sp., NL; 2-4. D. binodosus, NL; 5. D. binodosus, NL (Au); 6. Chiasmolithus consuetus, NL $(\mathrm{Au})$. 7. C. consuetus, $\mathrm{XN} ;$ 8. C. consuetus, NL, 9. C. consuetus, $\mathrm{XN}$. 

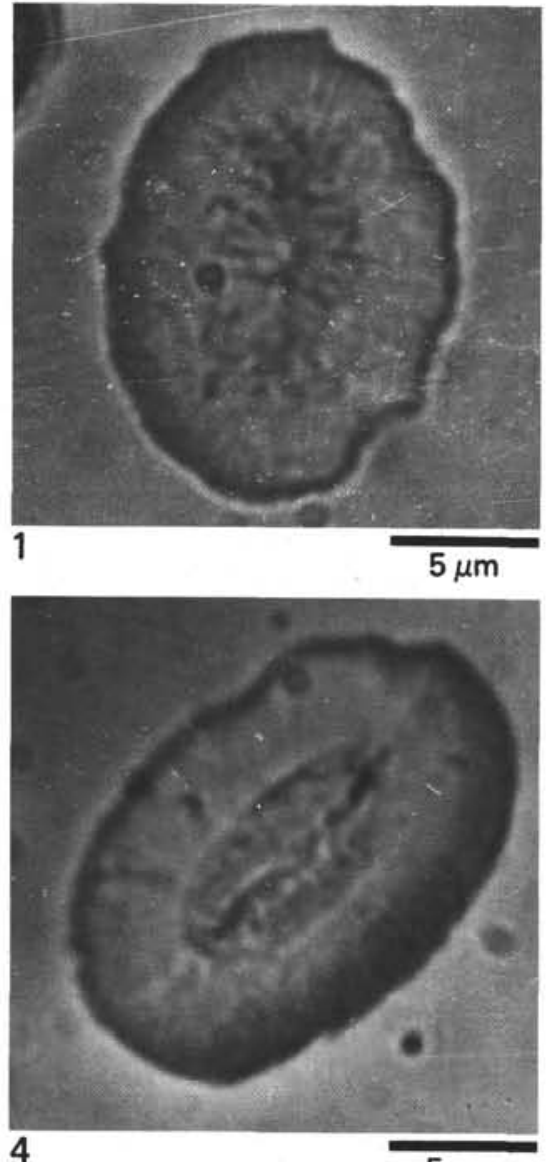$$
4
$$

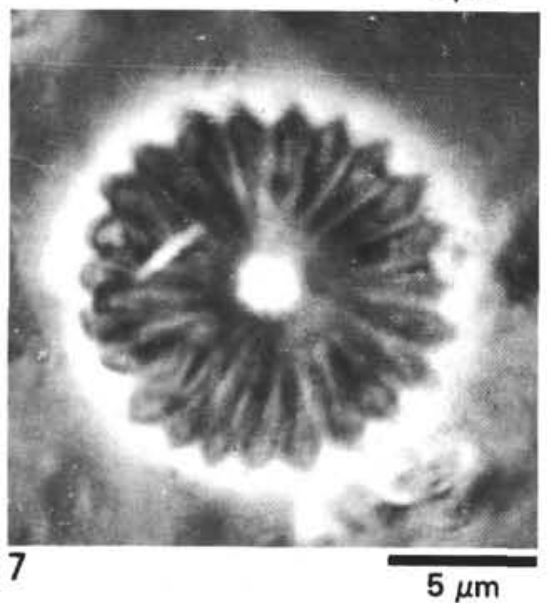

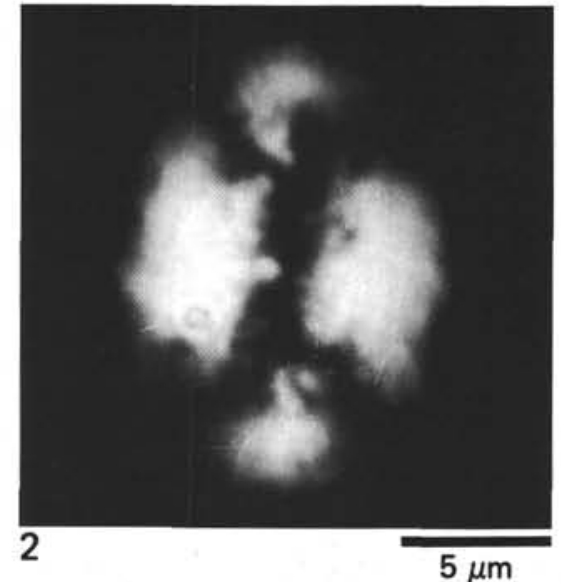
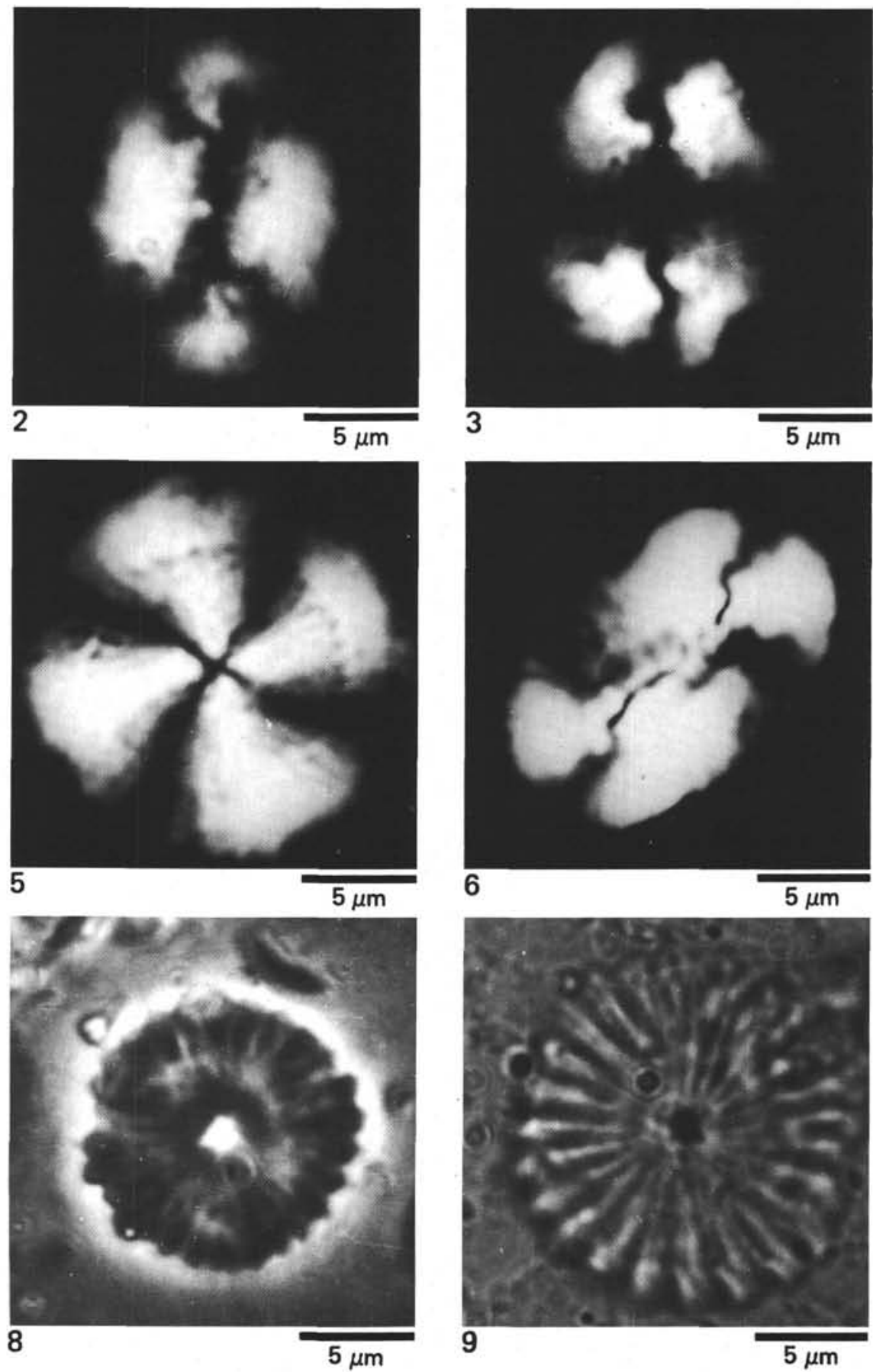
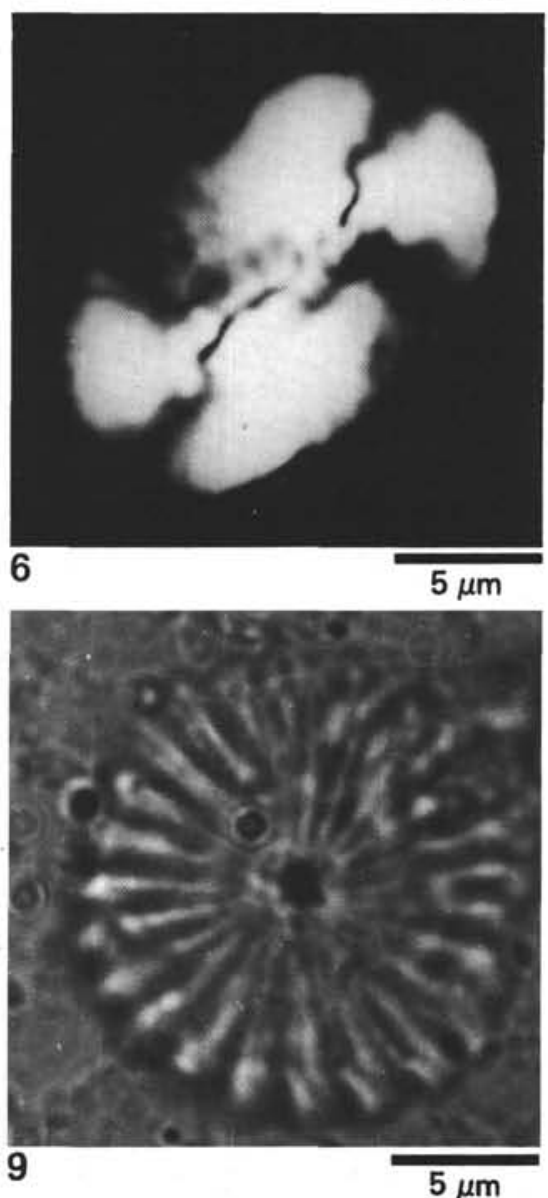

Plate 34. 1. Ellipsolithus macellus, Sample 530A-43-2, 20-21 cm, NL (Au); 2-3. E. macellus, Sample 530A-43-2, 20-21 cm, XN; 4. E. macellus, Sample 530A-43-2, 20-21 cm, NL (Au); 5. Heliolithus kleinpellii, Sample 530A-45-1, 57-58 cm, XN; 6. E. macellus, Sample 530A-43-2, 20-21 cm, XN; 7. Discoaster lenticularis, Sample 530A-41-1, 33-34 cm, NL; 8. D. lenticularis, Sample 530A-41-1, 33-34 cm, PHC; 9. D. multiradiatus, Sample $530 \mathrm{~A}-41-1,33-34 \mathrm{~cm}$, NL. 

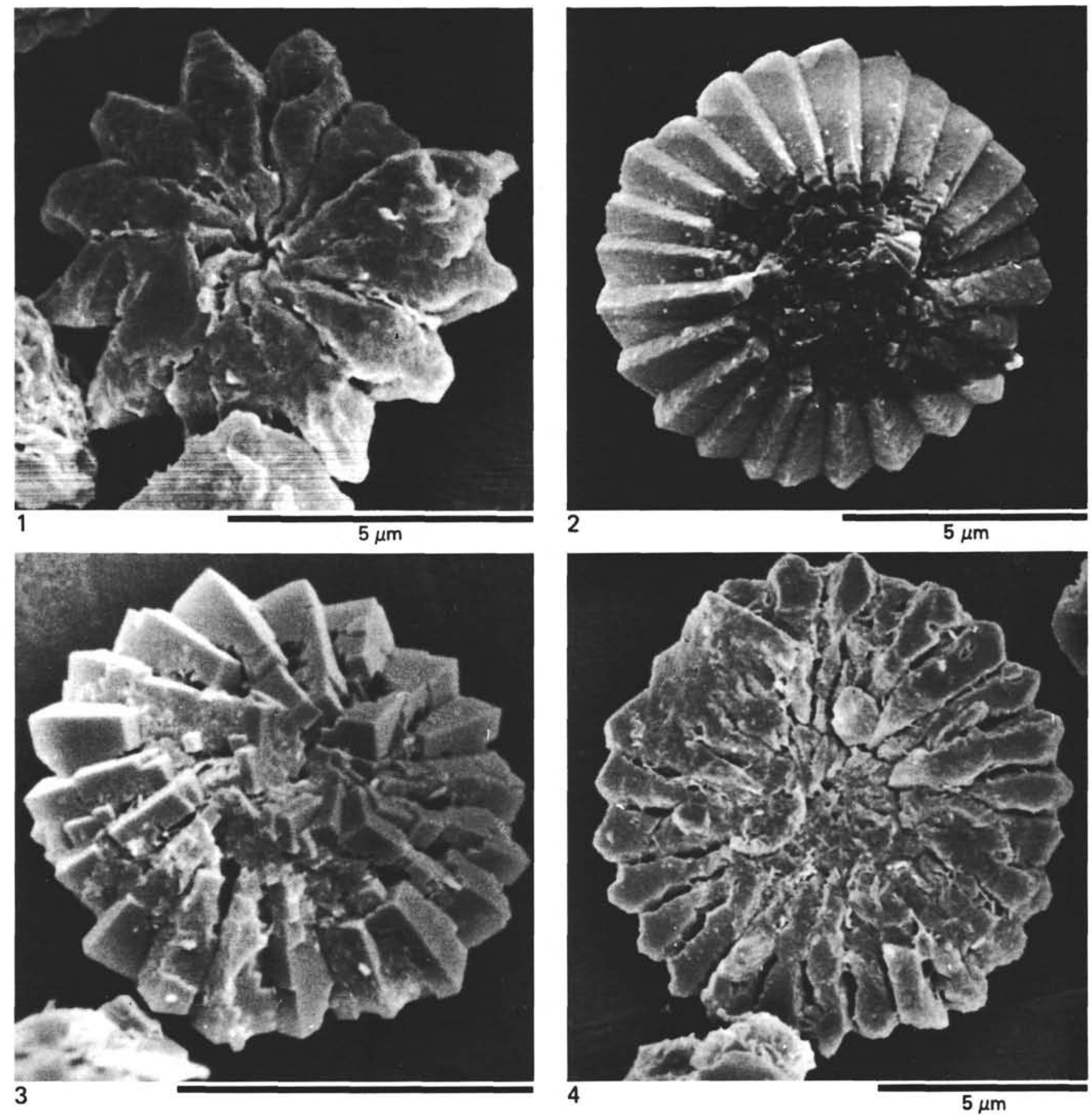

Plate 35. 1. Discoaster salisburgensis, Sample 530A-41,CC, SEM; $\quad$ 2. D. multiradiatus, Sample 530A-39-2, 21-22 cm, SEM; 3-4. D. multiradiatus, Sample 530A-41,CC, SEM. 

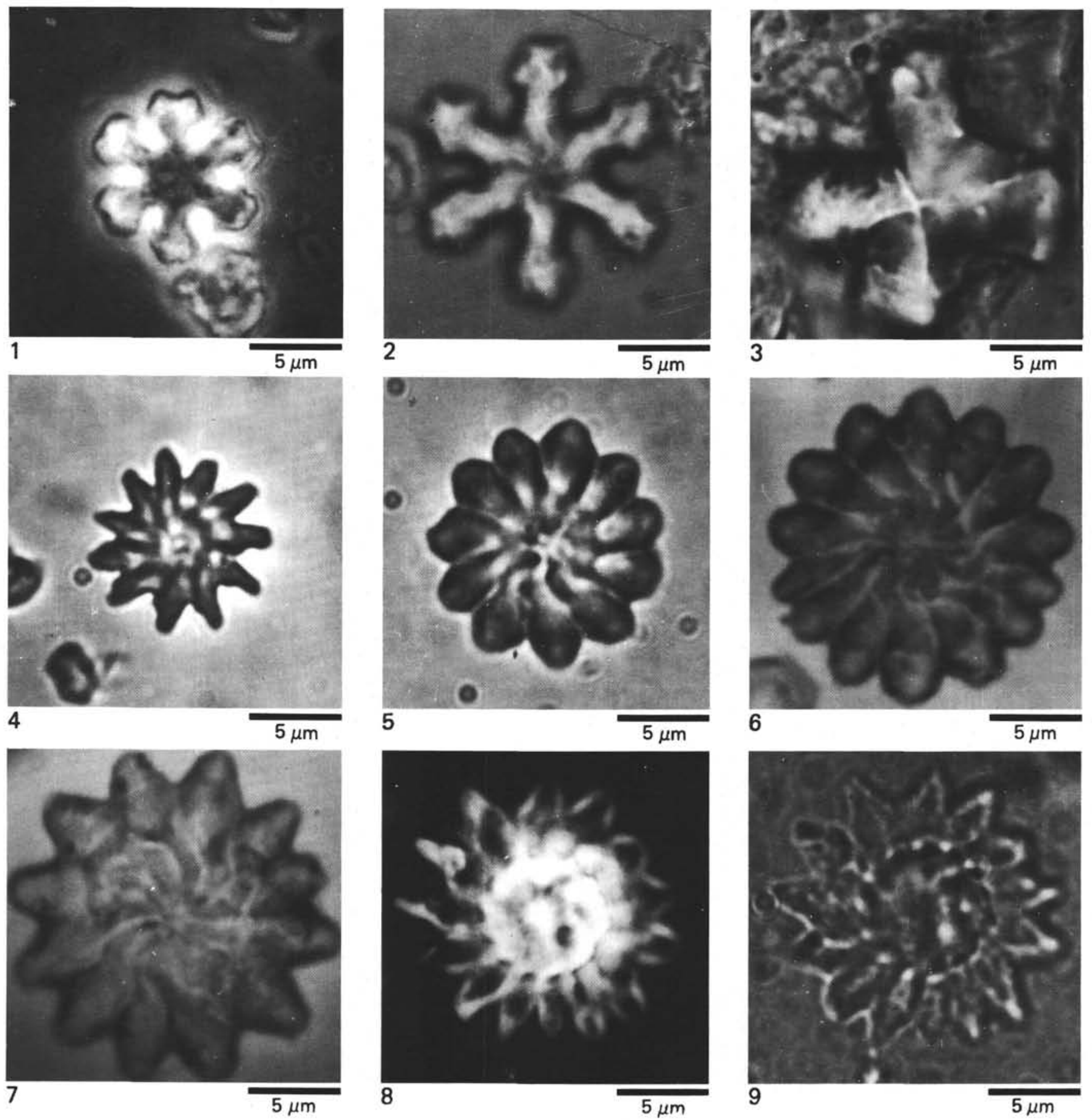

Plate 36. 1. Discoaster deflandrei, Sample 530A-35-1, 30-31 cm, PHC; 2. D. distinctus, Sample 530A-43-2, 20-21 cm, NL; 3. Nannotetrina swasticoides, Sample 530A-38-1, 39-40 cm, NL; 4. Discoaster sp., Sample 530A-43-2, 20-21 cm, NL (Au); 5-7. D. salisburgensis, Sample 530A-43-2, 20-21 cm, NL (Au); 8. D. splendidus, Sample 530A-43-1, 15-16 cm, XN; 9. D splendidus, Sample 530A-43-1, 15-16 cm, NL. 

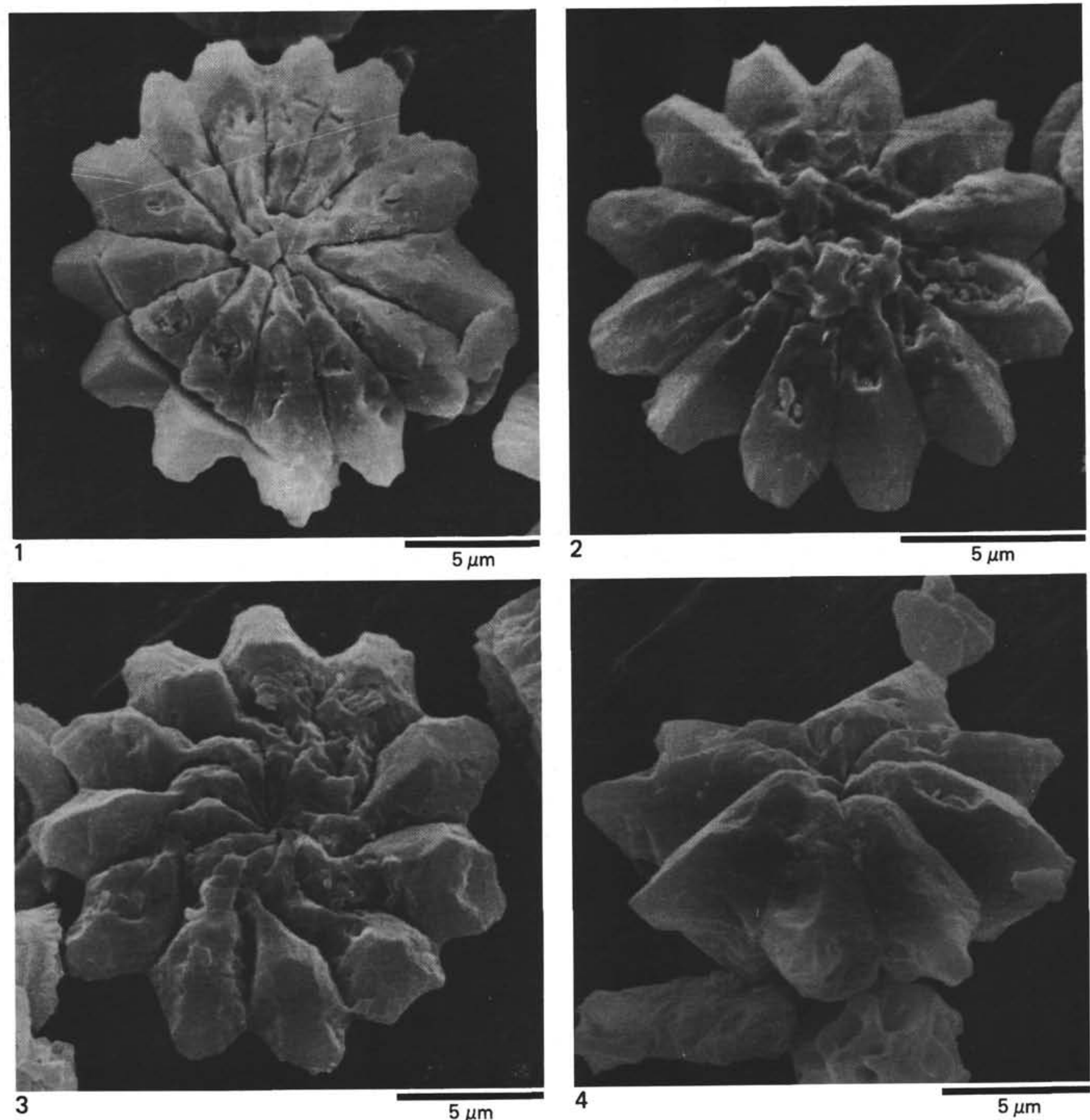

Plate 37. 1-3. Discoaster salisburgensis, Sample 530A-43-2, 20-21 cm, SEM; 4. D. salisburgensis, side view, Sample 530A-43-2, 20-21 cm, SEM. 


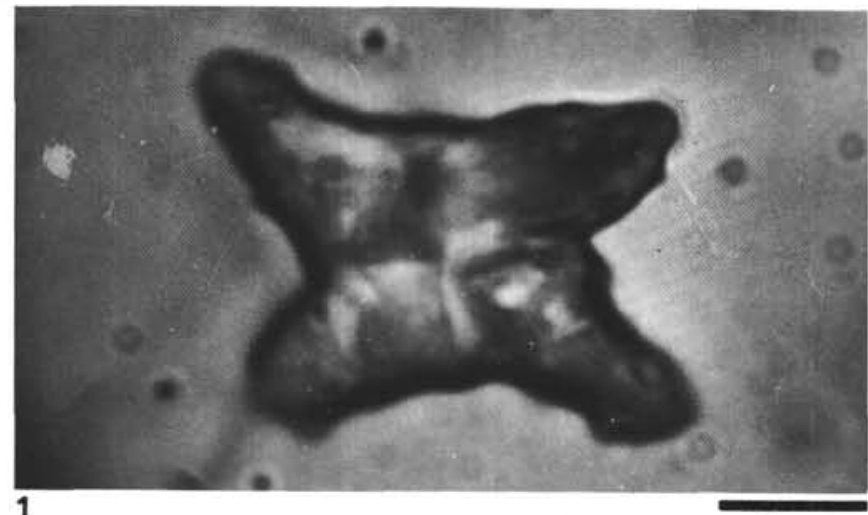

1
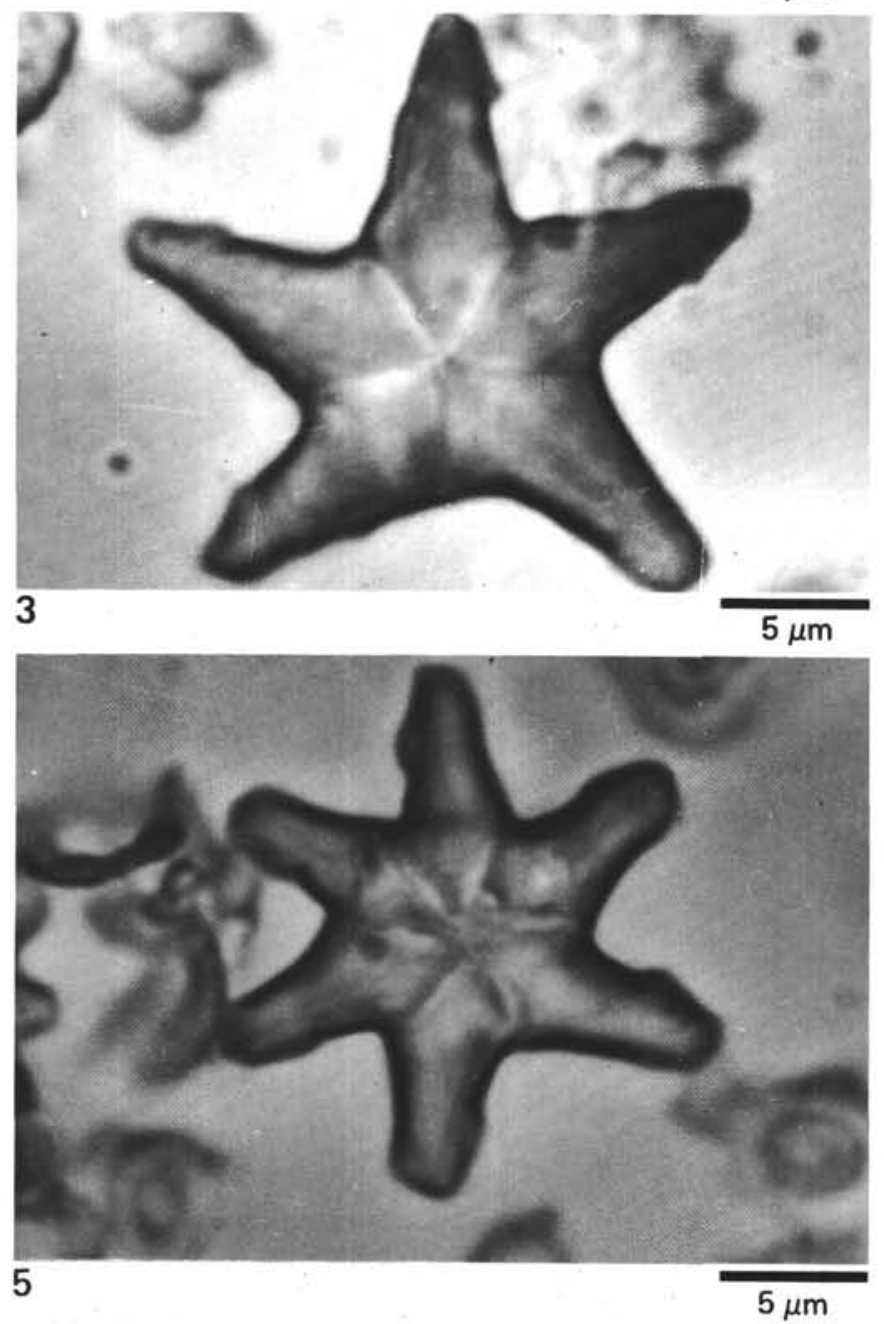

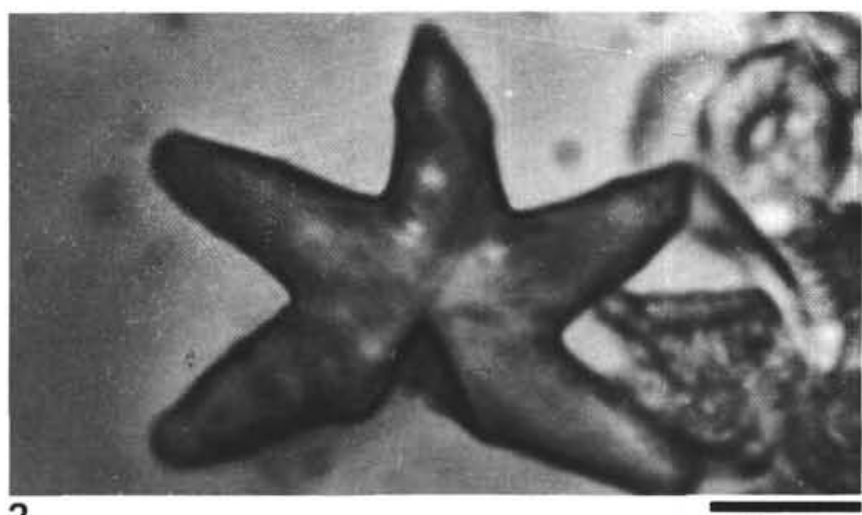

2

$5 \mu \mathrm{m}$
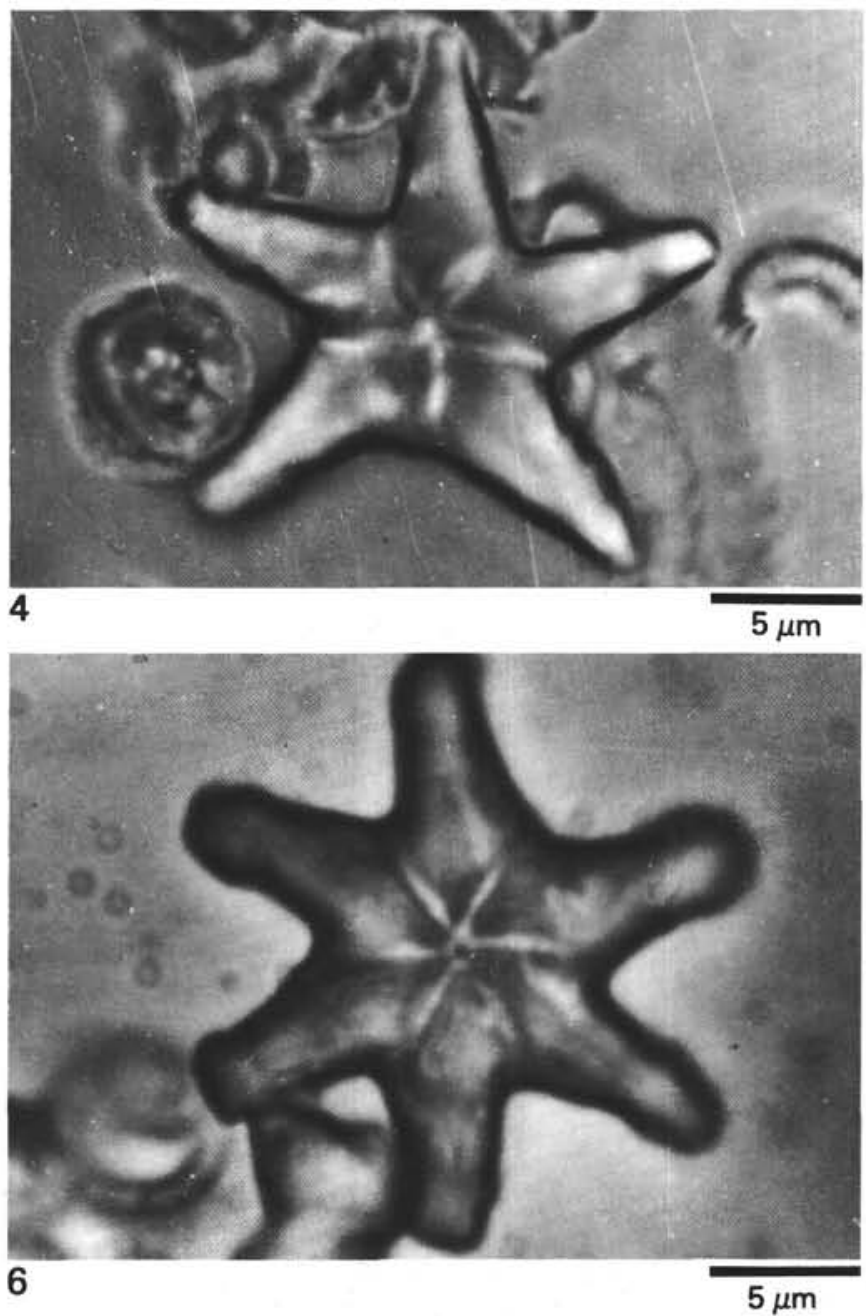

Plate 38. 1-6. Discoaster okadai, Sample 530A-43-2, 20-21 cm, NL (Au). 

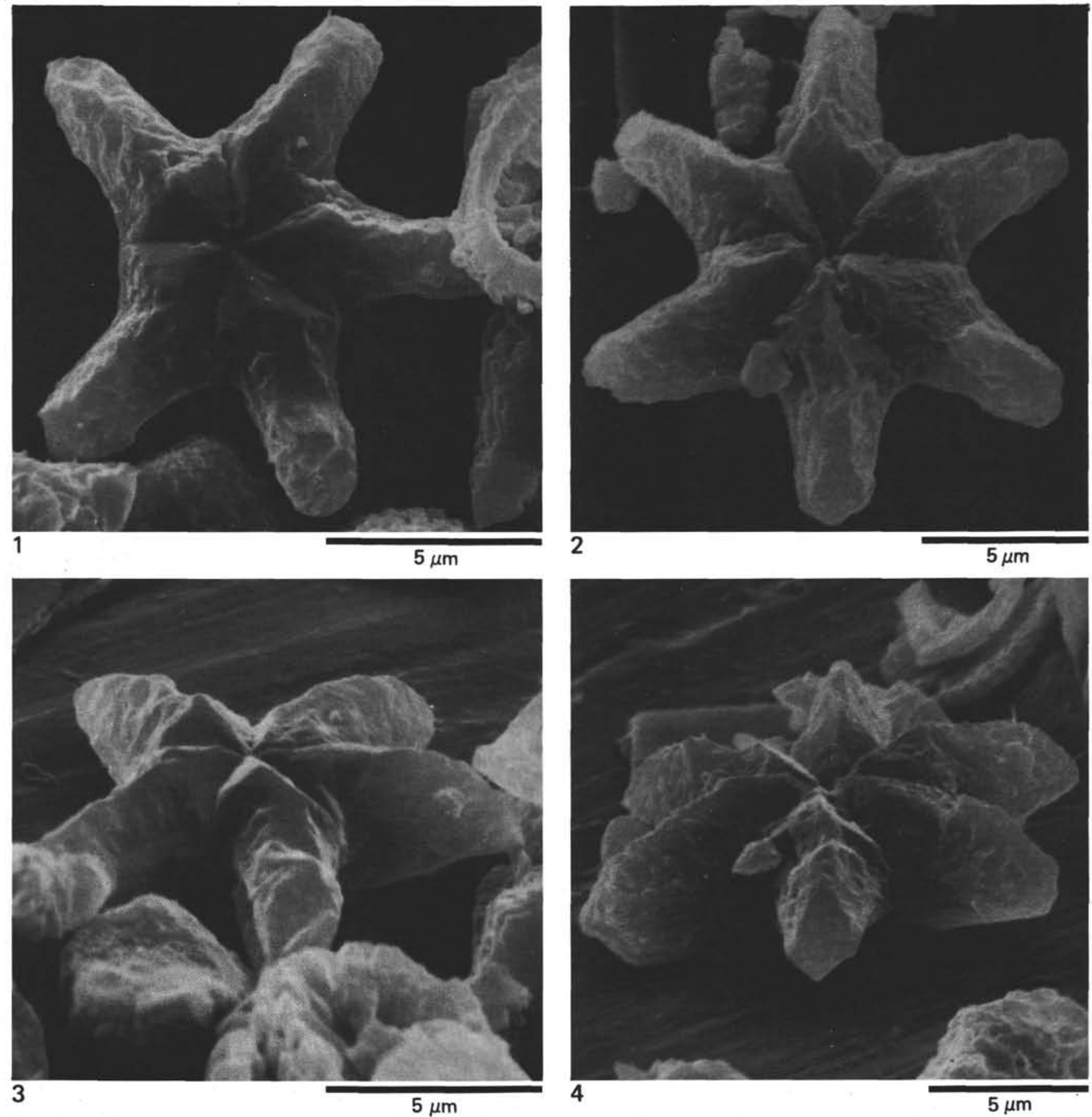

Plate 39. 1-4. Discoaster okadai, Sample 530A-43-2, 20-21 cm, SEM. 


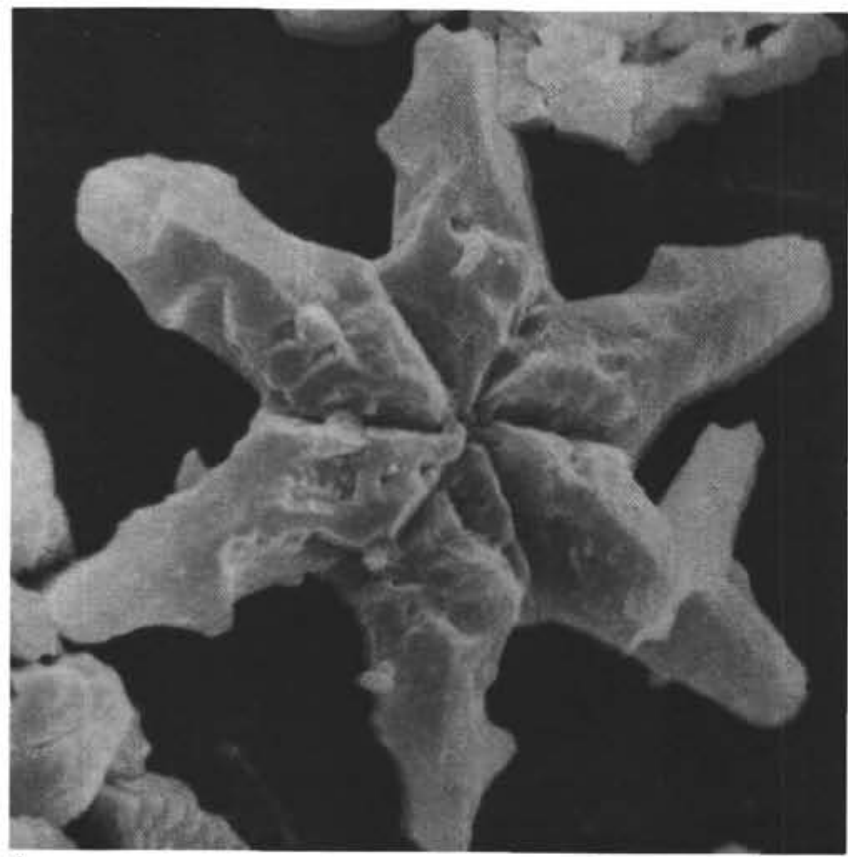

1

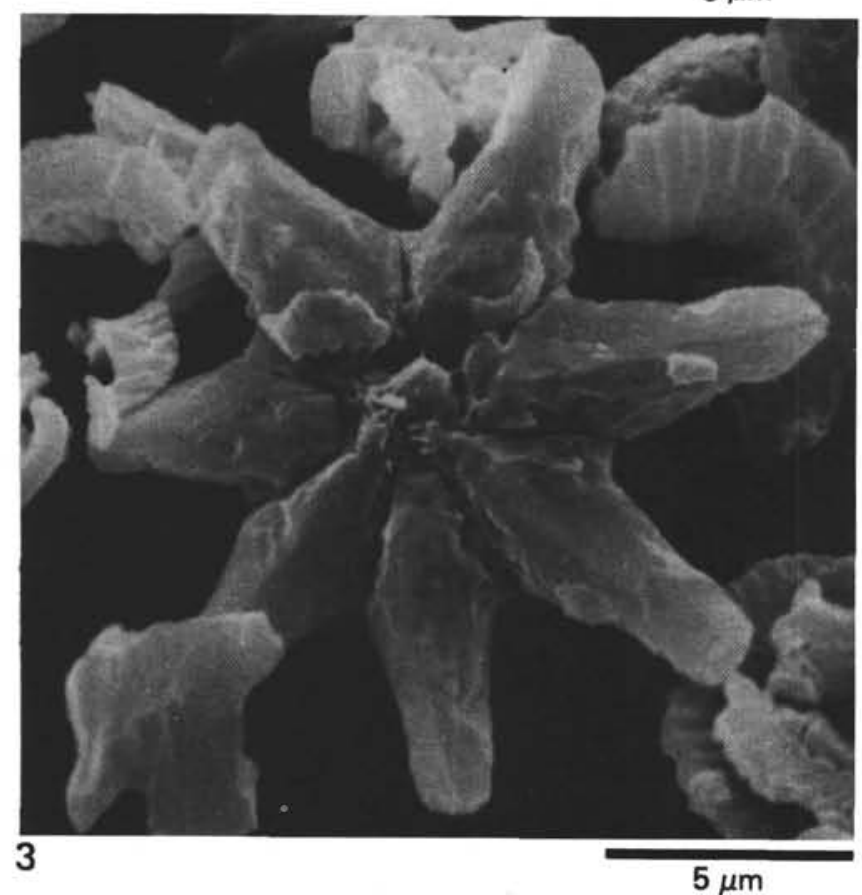

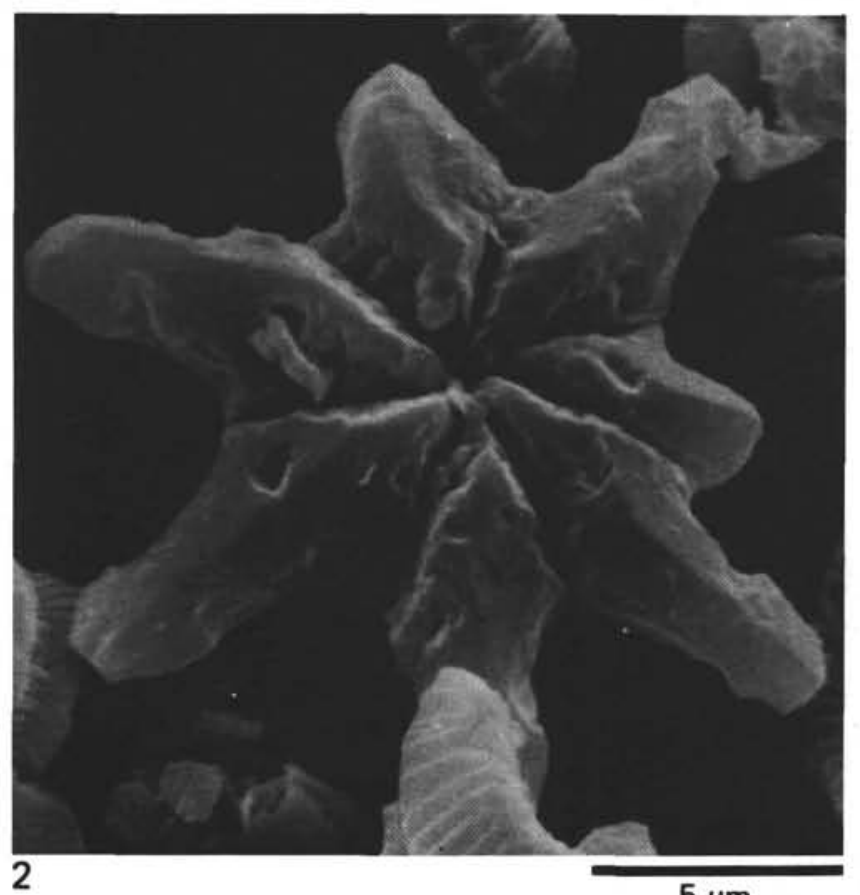

$5 \mu \mathrm{m}$

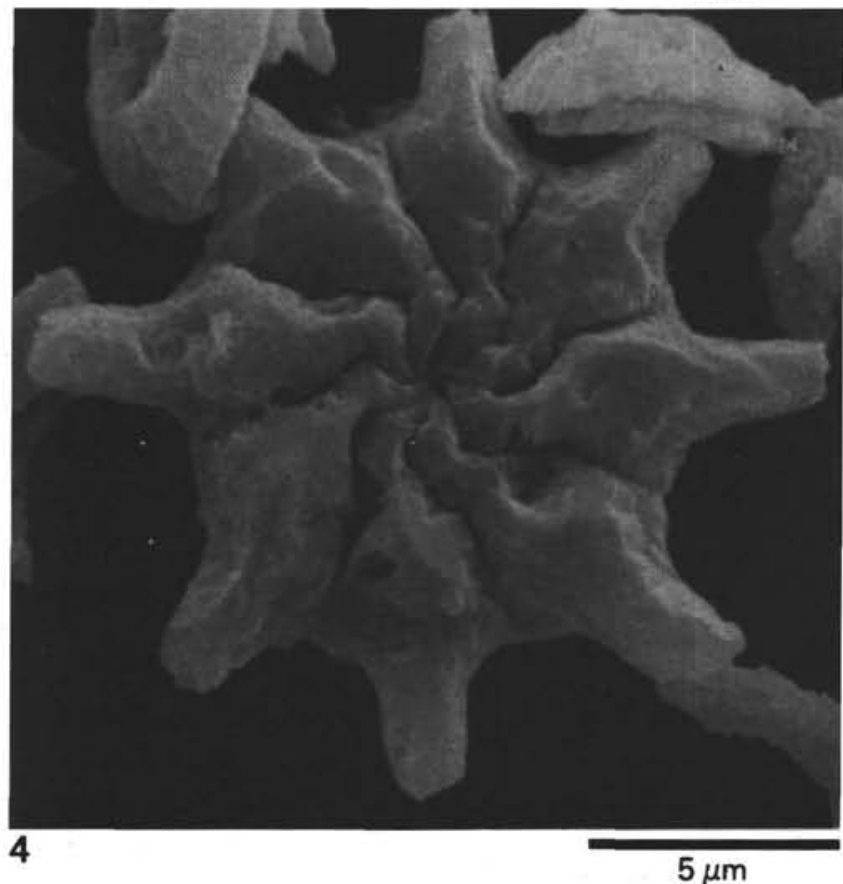

$5 \mu \mathrm{m}$

Plate 40. 1-3. Discoaster okadai, Sample 530A-43-2, 20-21 cm, SEM; 4. D. mediosus, Sample 530A-43-2, 20-21 cm, SEM. 

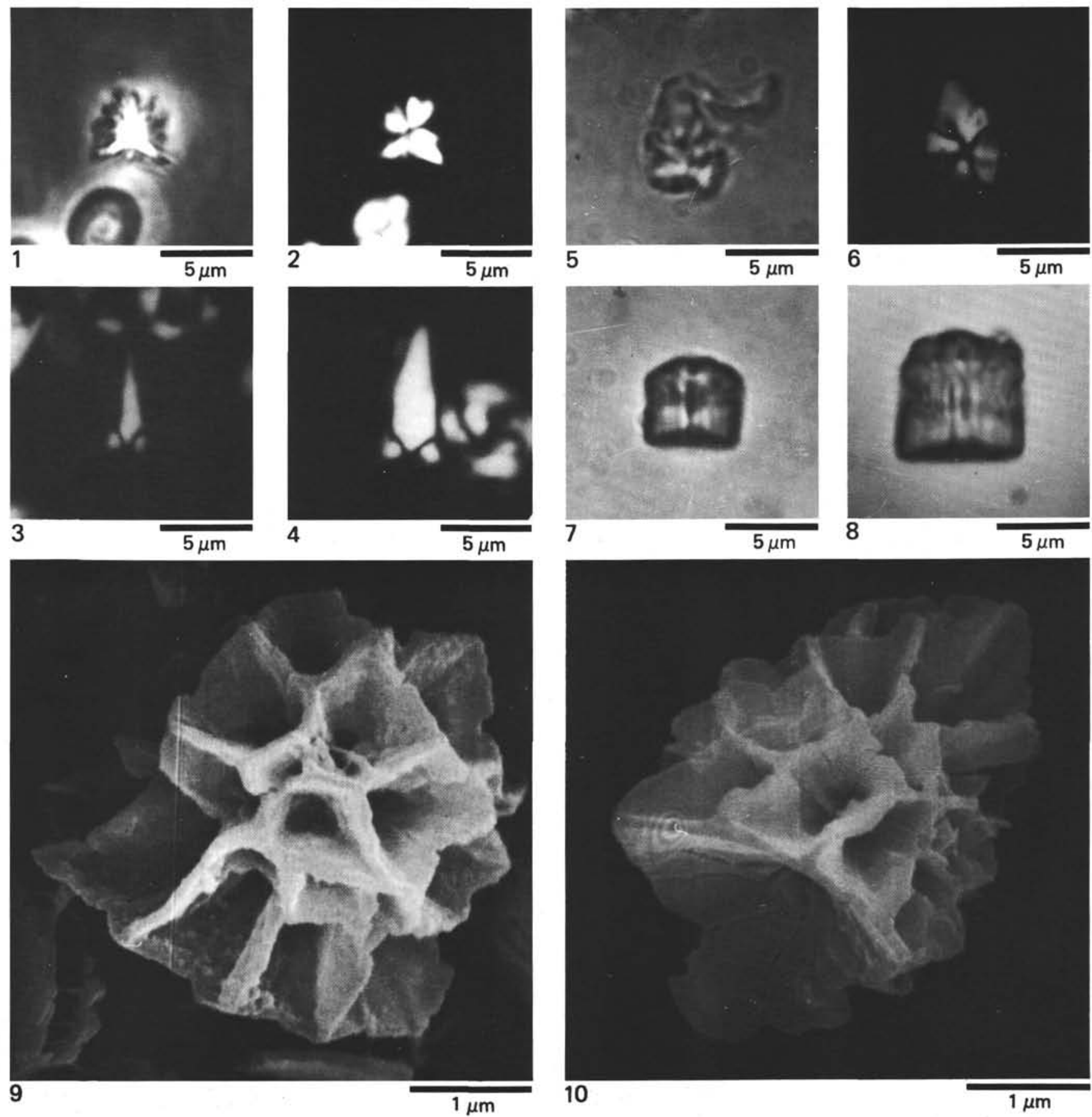

Plate 41. 1. Sphenolithus abies, Sample 532-55,CC, PHC; 2. S. abies, Sample 532-55,CC, XN; 3. S. distentus, Sample 530A-37-2, 1-2 cm, $\mathrm{XN}$; 4. $S$. distentus, Sample $530 \mathrm{~A}-37-1,80-81 \mathrm{~cm}$, XN; 5. S. radians, Sample 530A-43,CC, NL; 6. S. radians, Sample 530A-43,CC, $\mathrm{XN}$; 7-8. Fasciculithus involutus, Sample 530A-43-2, 20-21 cm, NL (Au); 9. S. abies, Sample 532-55,CC, SEM; 10. S. abies, Sample $532-46, C C$, SEM. 


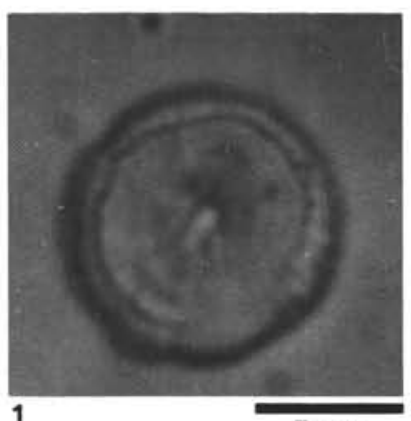

1

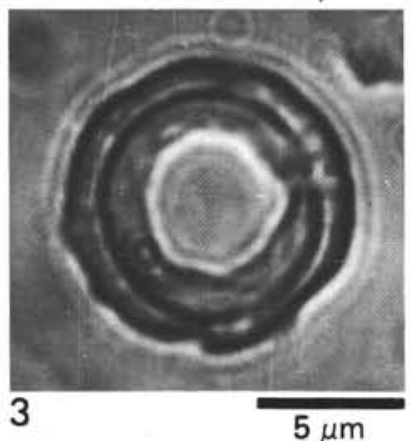

$5 \mu \mathrm{m}$
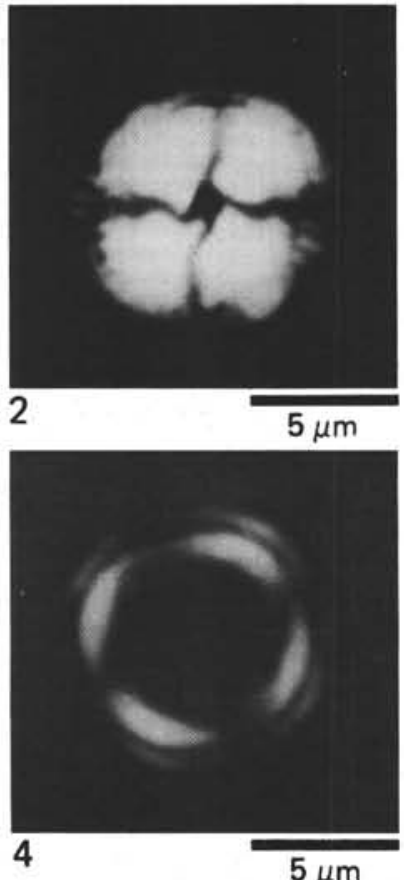

$5 \mu \mathrm{m}$

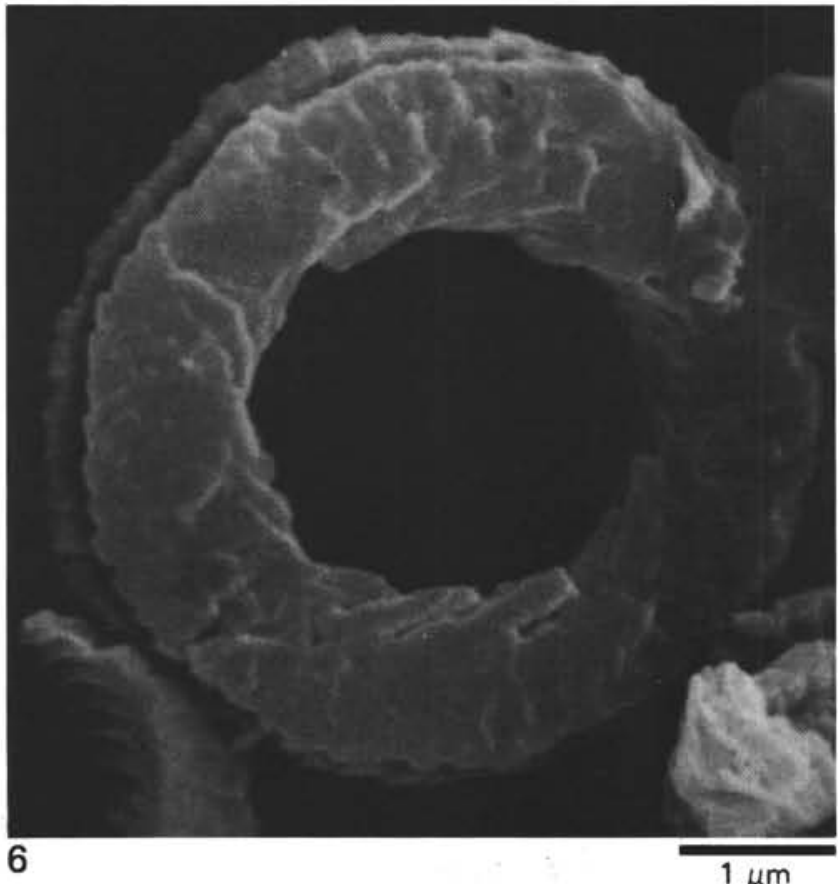

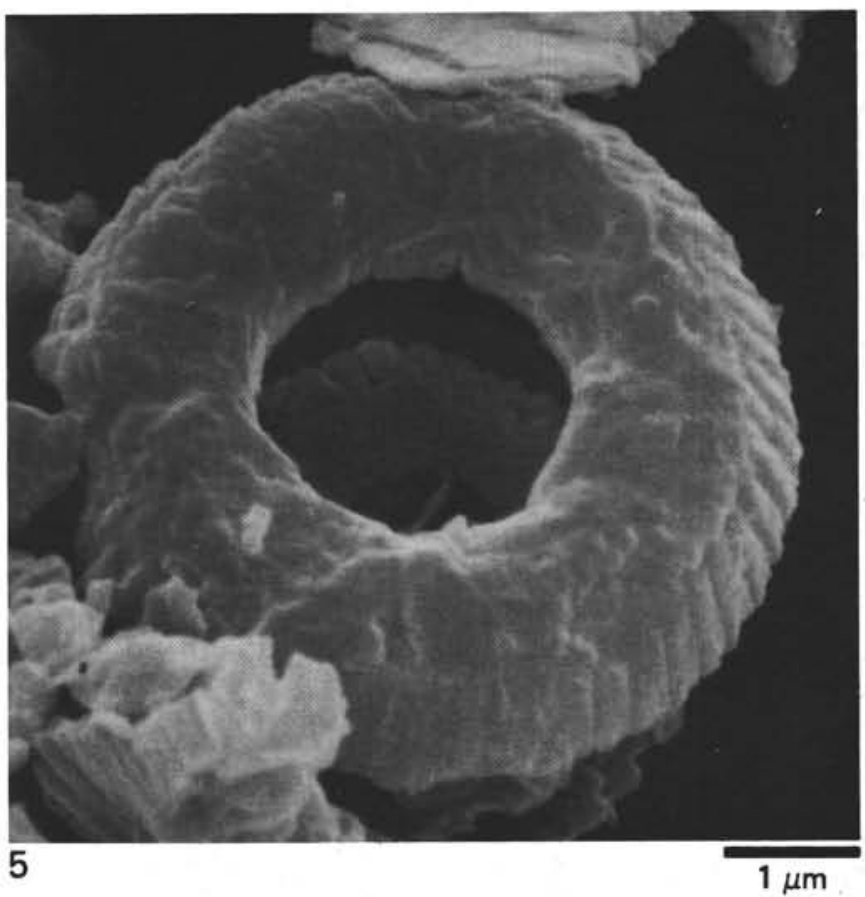

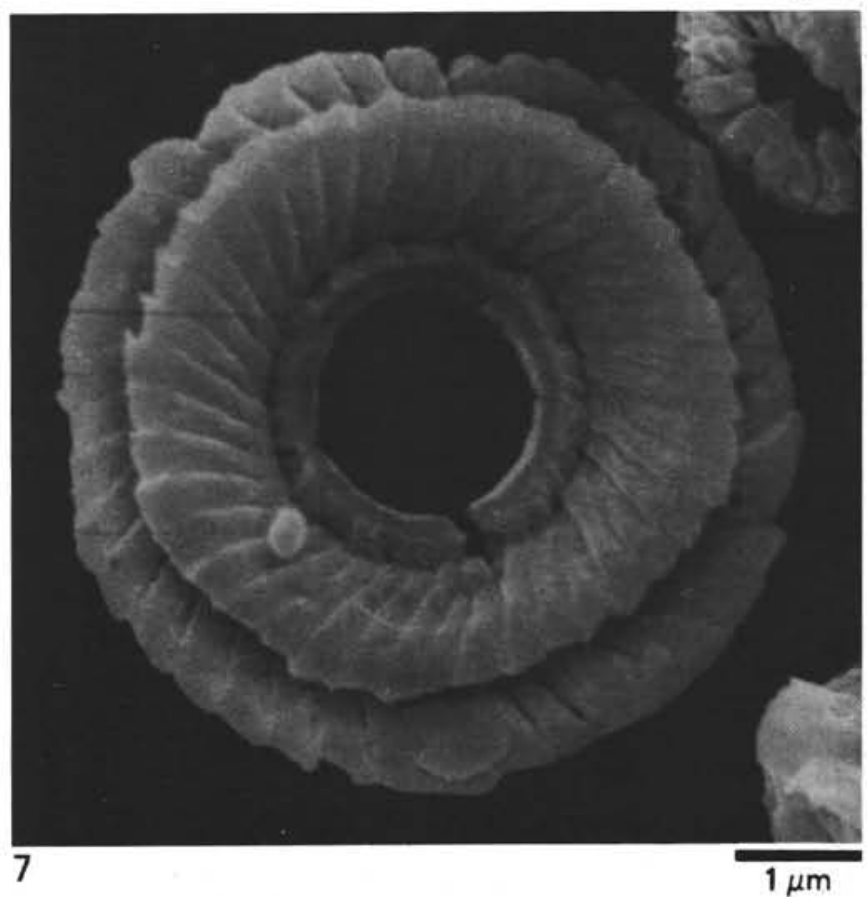

Plate 42. Sample 530A-43-2, 20-21 cm. 1. Coccolithus crassus, NL (Au); 2. C. crassus, XN; 3. Cyclolithella robusta, NL (Au); 4. C. robusta, XN; 5. C. robusta, SEM; 6. C. robusta, SEM; 7. Calcidiscus formosus, proximal view, SEM. 


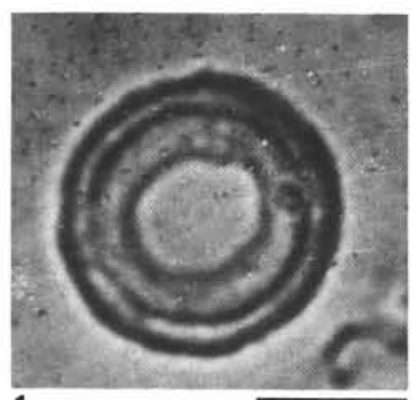

1

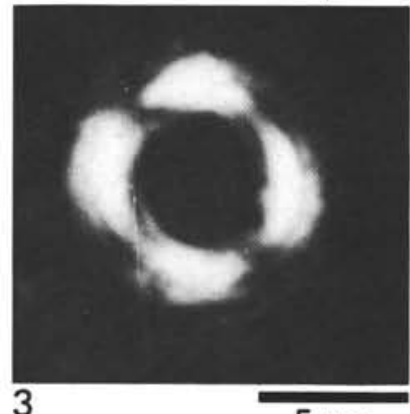

$5 \mu \mathrm{m}$
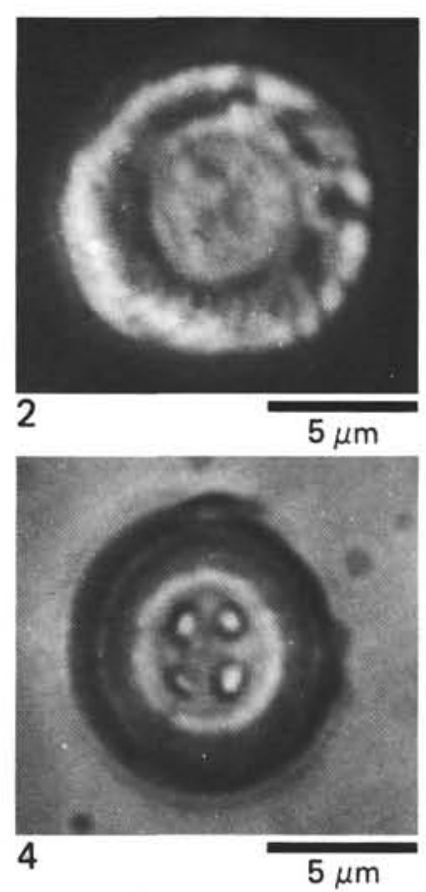

$5 \mu \mathrm{m}$
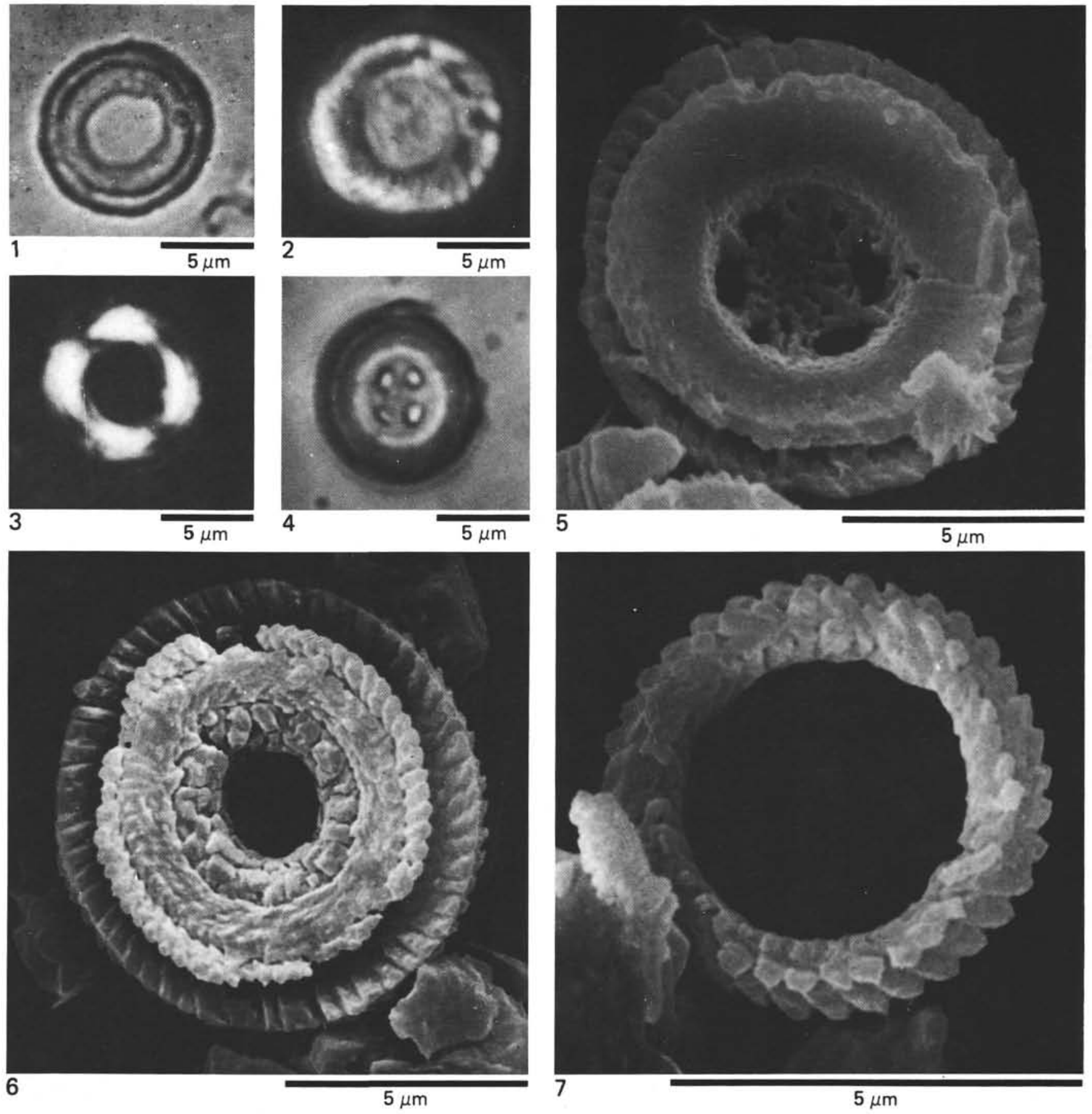

Plate 43. 1. Cyclolithella robusta, Sample 530A-43-2, $20-21 \mathrm{~cm}$, NL (Au); 2. C. robusta, Sample 530A-43-2, 20-21 cm, PHC;3. C. robusta, Sample 530A-43-2, 20-21 cm, XN; 4. Toweius eminens, Sample 530A-43-2, 20-21 cm, NL (Au); 5. Toweius cf. T. craticulus, proximal view, Sample 530A-43-2, 20-21 cm, SEM; 6. Ericsonia cava, proximal view, Sample 530A-37-1, 96-97 cm, SEM; 7. Cyclolithella prionion, Sample $530 \mathrm{~A}-37-1,96-97 \mathrm{~cm}, \mathrm{SEM}$. 


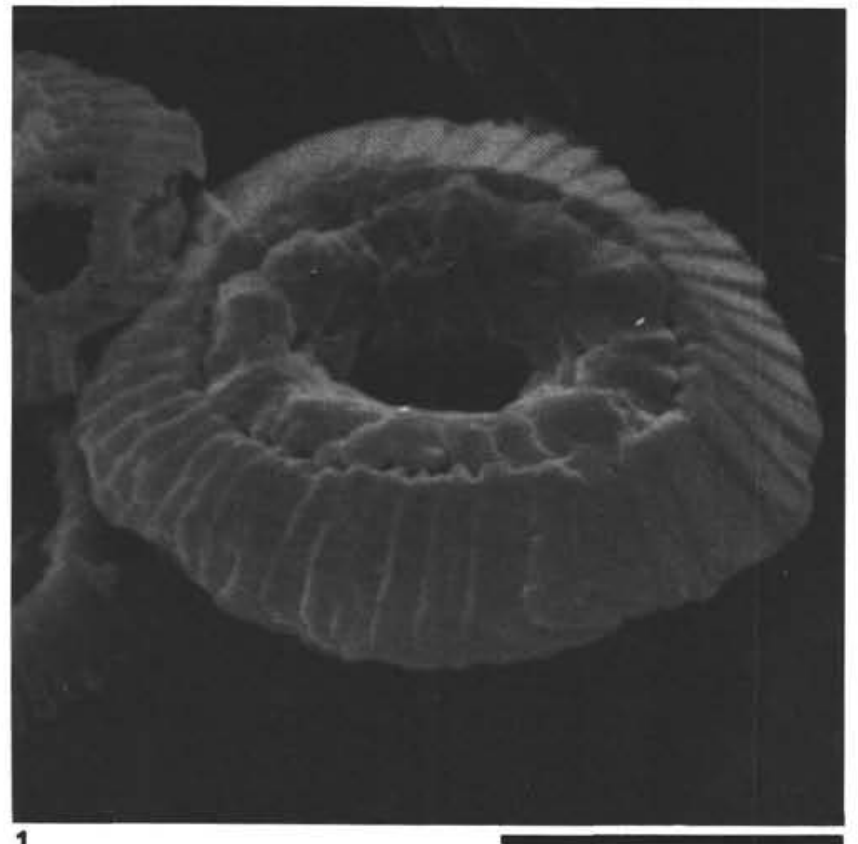

1

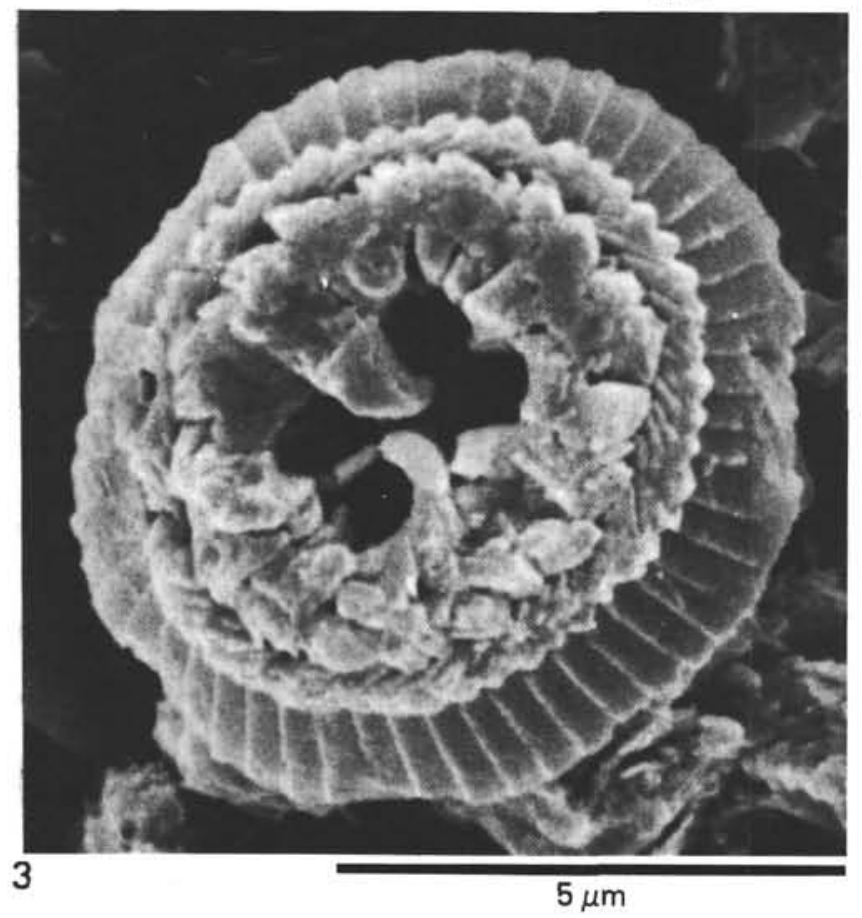

Plate 44. Sample 530A-43-2, 20-21 cm. 1. Ericsonia cava, distal view, SEM; view, SEM; 4. T. eminens, proximal view, SEM.
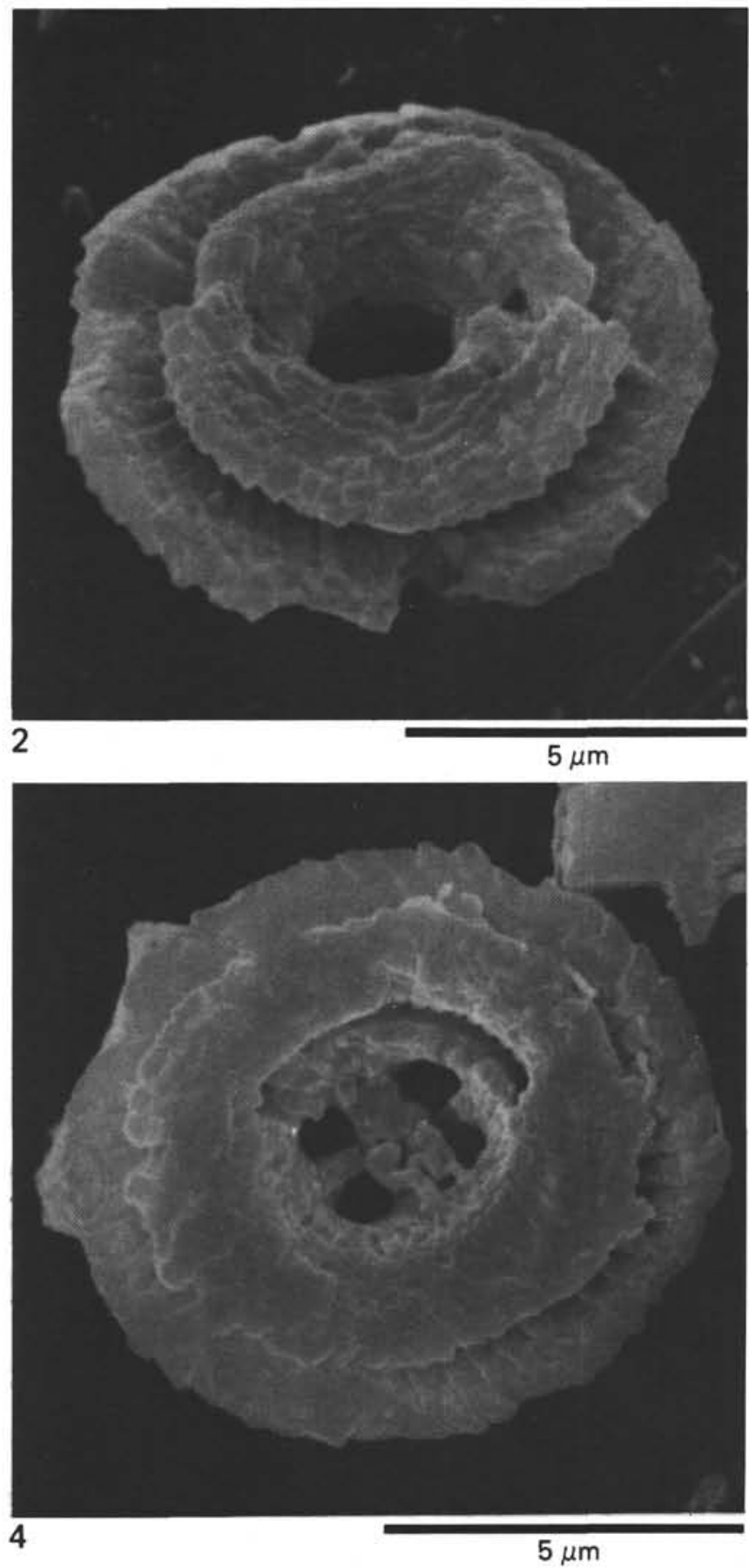

2. E. cava, proximal view, SEM; 3. Toweius eminens, distal 

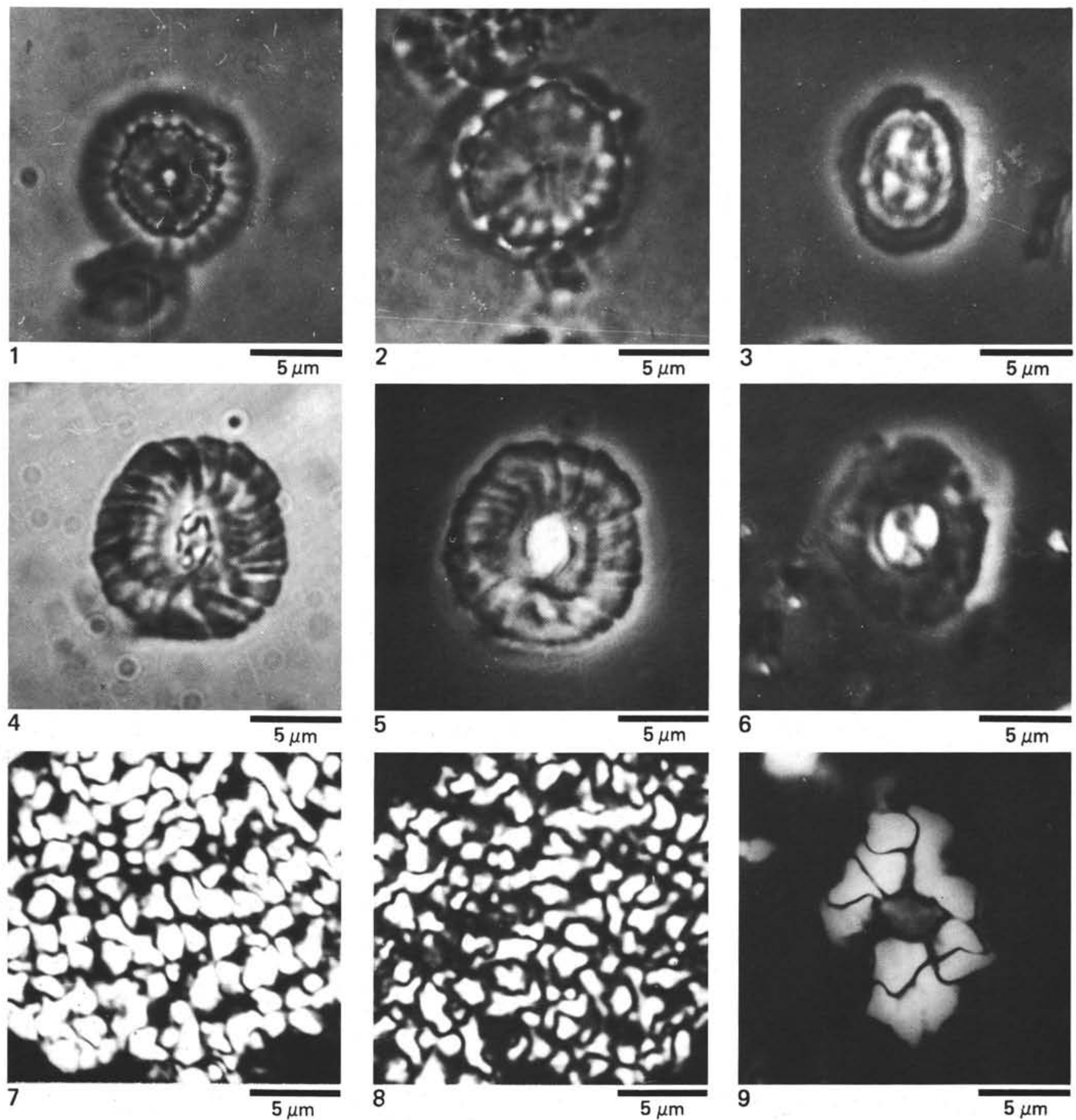

Plate 45. 1-2. Heliolithus cantabriae, Sample 530A-43,CC, NL; 3. Chiasmolithus danicus, Sample 530A-47-1, 142-143 cm, PHC; 4. Biscutum cf. B. castrorum, Sample 530A-49-1, 73-74 cm, NL; 5. Biscutum cf. B. castrorum, Sample 530A-49-1, 73-74 cm, PHC; 6. Markalius astroporus, Sample 530A-50-1, 6-7 cm, PHC; 7-8. Thoracosphaera operculata, Sample 530A-50-2, 13-14 cm, XN; 9. Thoracosphaera sax$e a$, Sample 530A-50-2, 13-14 cm, XN. 

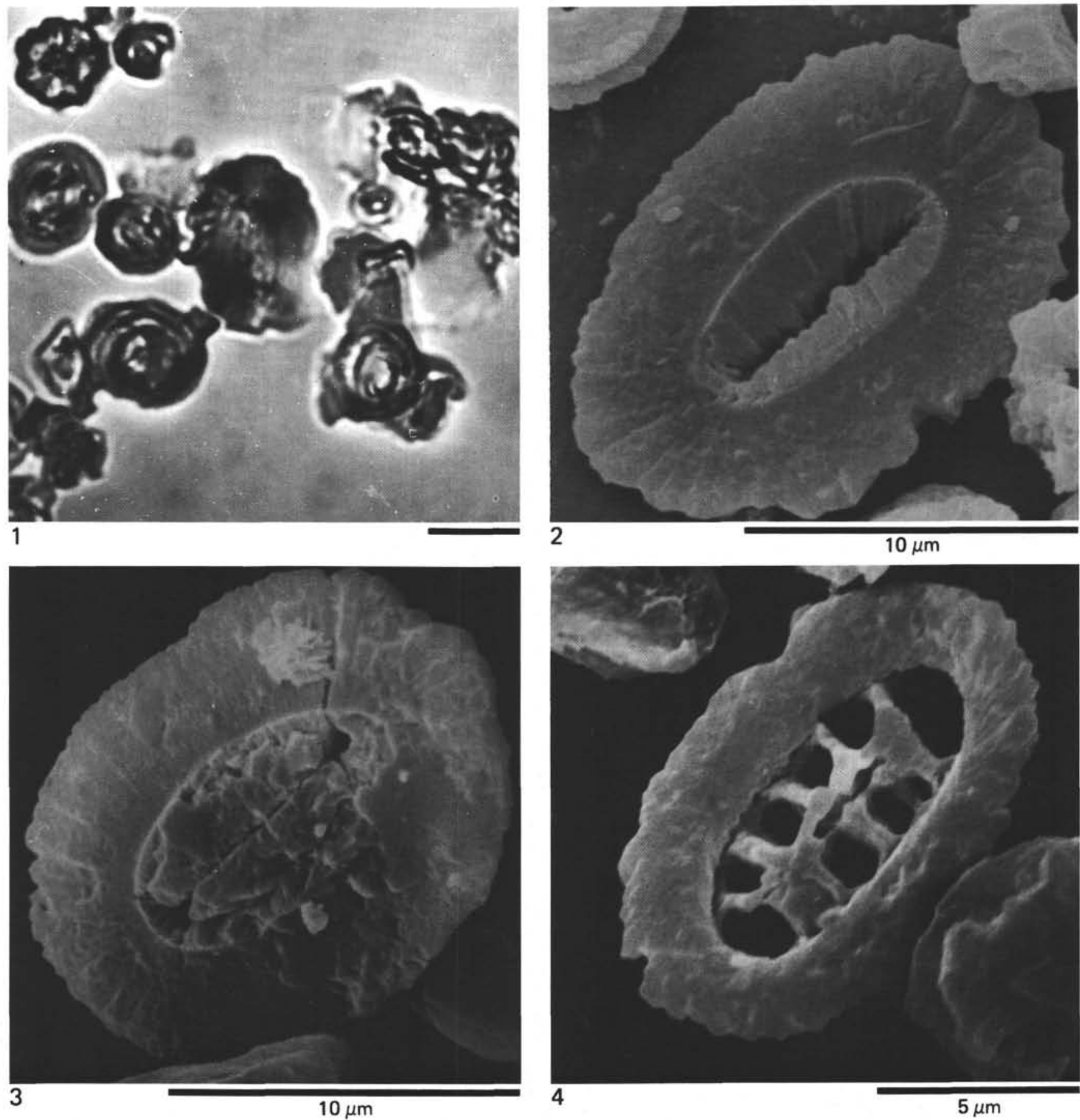

Plate 46. Sample 530A-43-2, 20-21 cm. 1. Ellipsolithus macellus, partly polarized (Au); 2. E. macellus, SEM; 3. E. macellus, SEM; 4. E. distichus, SEM. 


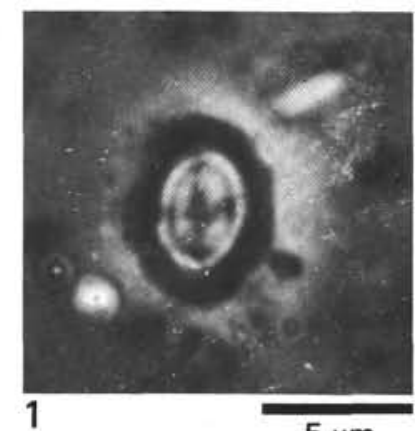

$$
1
$$
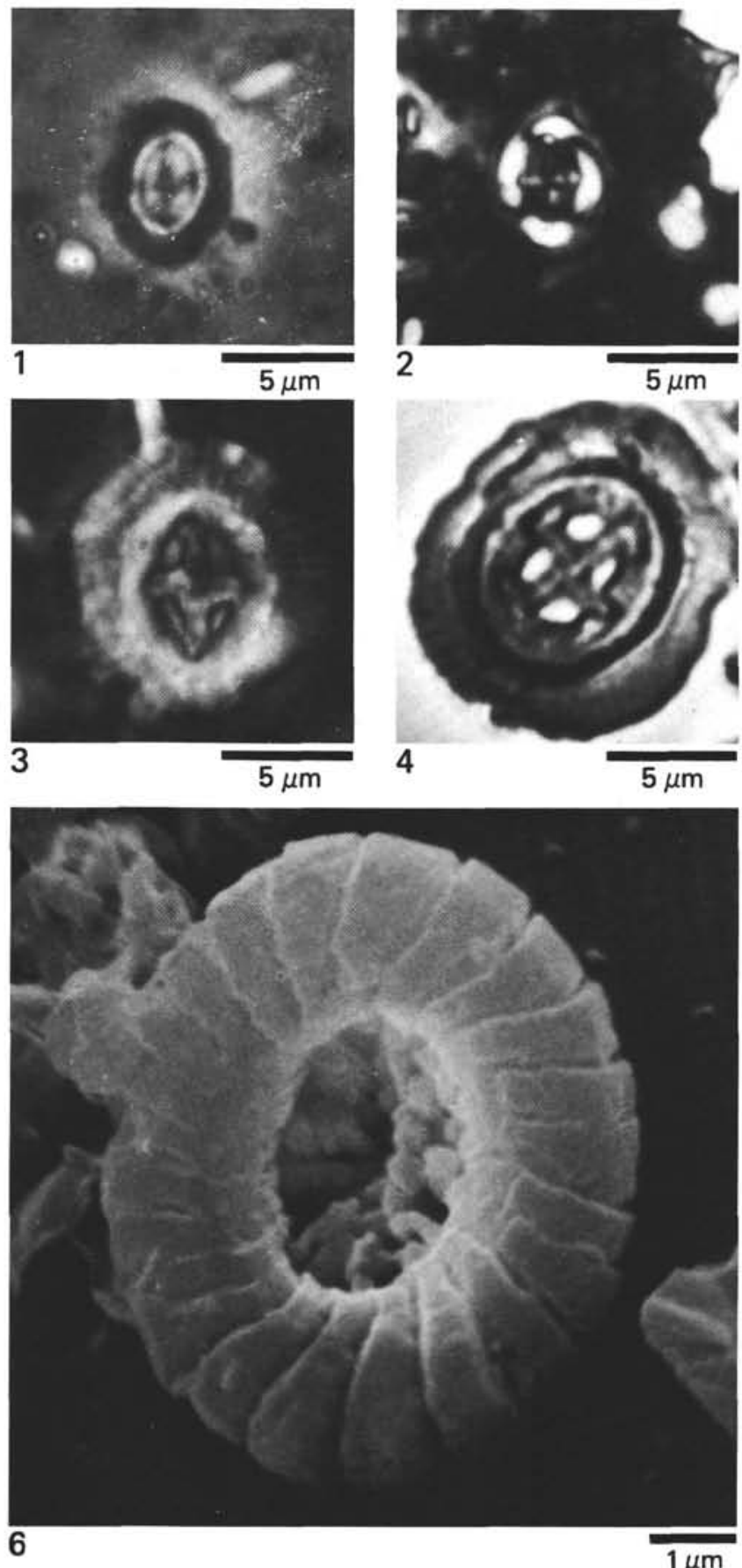

$5 \mu \mathrm{m}$

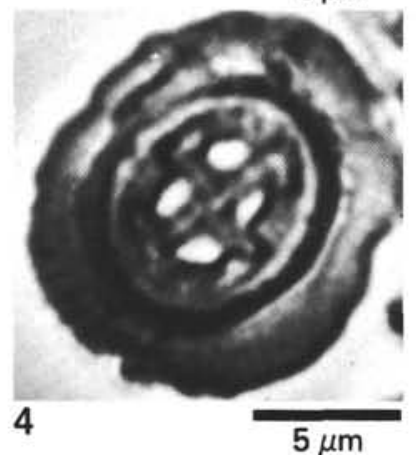

5

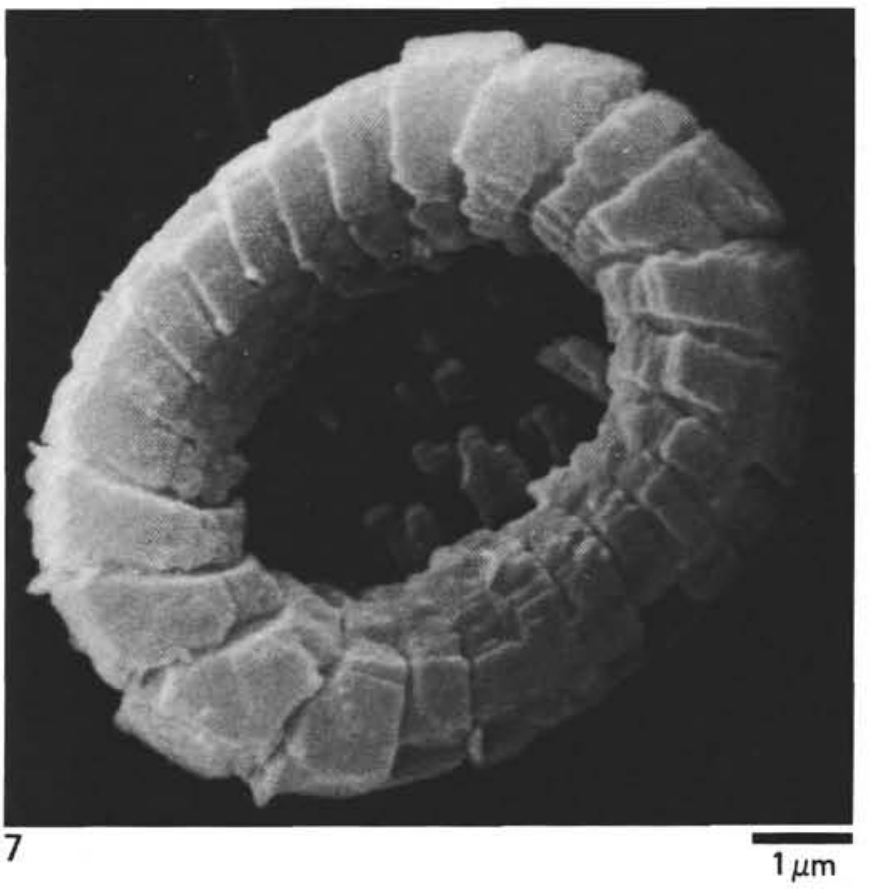

Plate 47. 1. Cruciplacolithus primus, Sample 530A-50-1, 6-7 cm, PHC; 2. C. primus, Sample 530A-50-1, 6-7 cm, XN; 3. C. tenuis, Sample 530A-47-1, 142-143 cm, PHC; 4. C. tenuis, Sample 530A-43-2, 20-21 cm, NL (Au); 5. C. edwardsii, distal view, Sample 530A-50-1, 6-7 cm, SEM; 6-7. Biscutum sp., Sample 530A-50-1, 6-7 cm, SEM. 


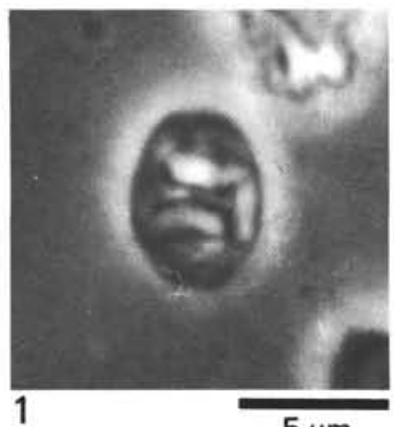

1

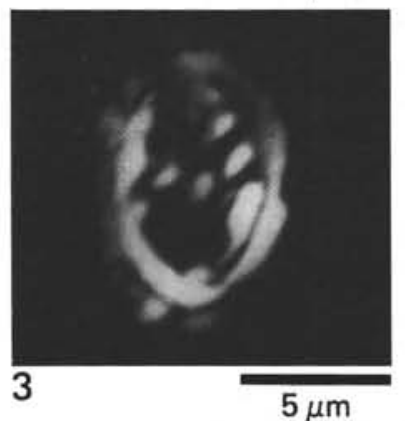

$5 \mu \mathrm{m}$
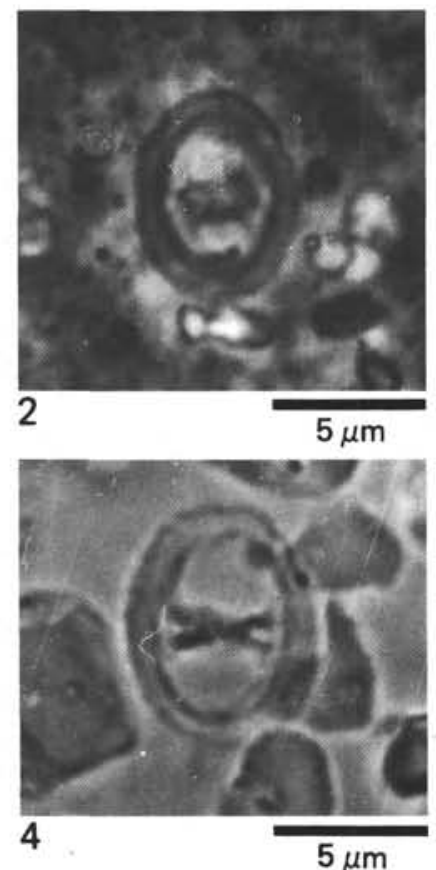

$5 \mu \mathrm{m}$
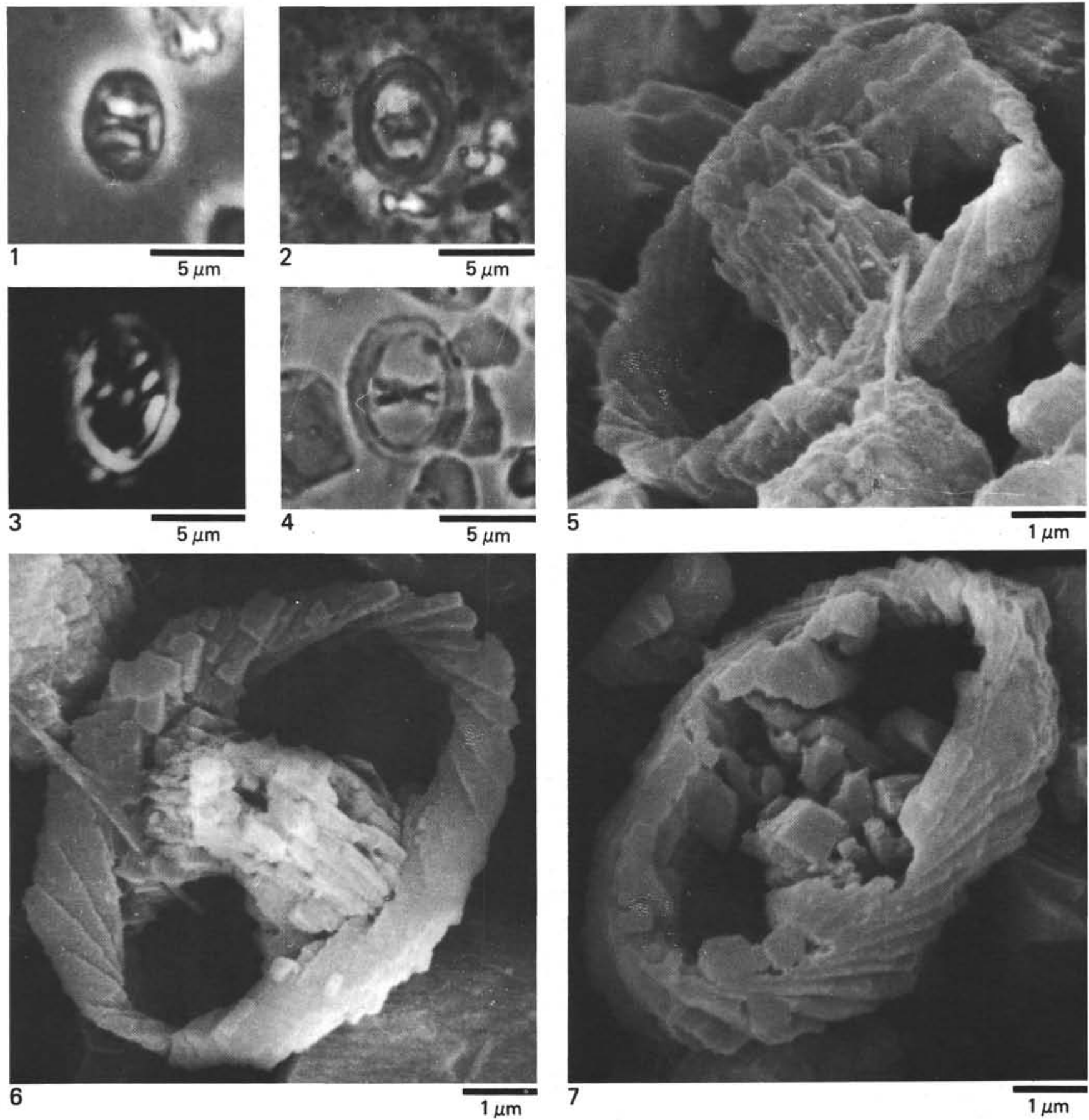

Plate 48. 1-2. Zygodiscus sigmoides, Sample 530A-50-1, 6-7 cm, PHC; 3. Z. sigmoides, Sample 530A-48-2, 43-44 cm, XN; 4. Neochiastozygus junctus, Sample 530A-50-2, 13-14 cm, NL; 5-7. Zygodiscus sigmoides, Sample 530A-50-1, 6-7 cm, SEM. 

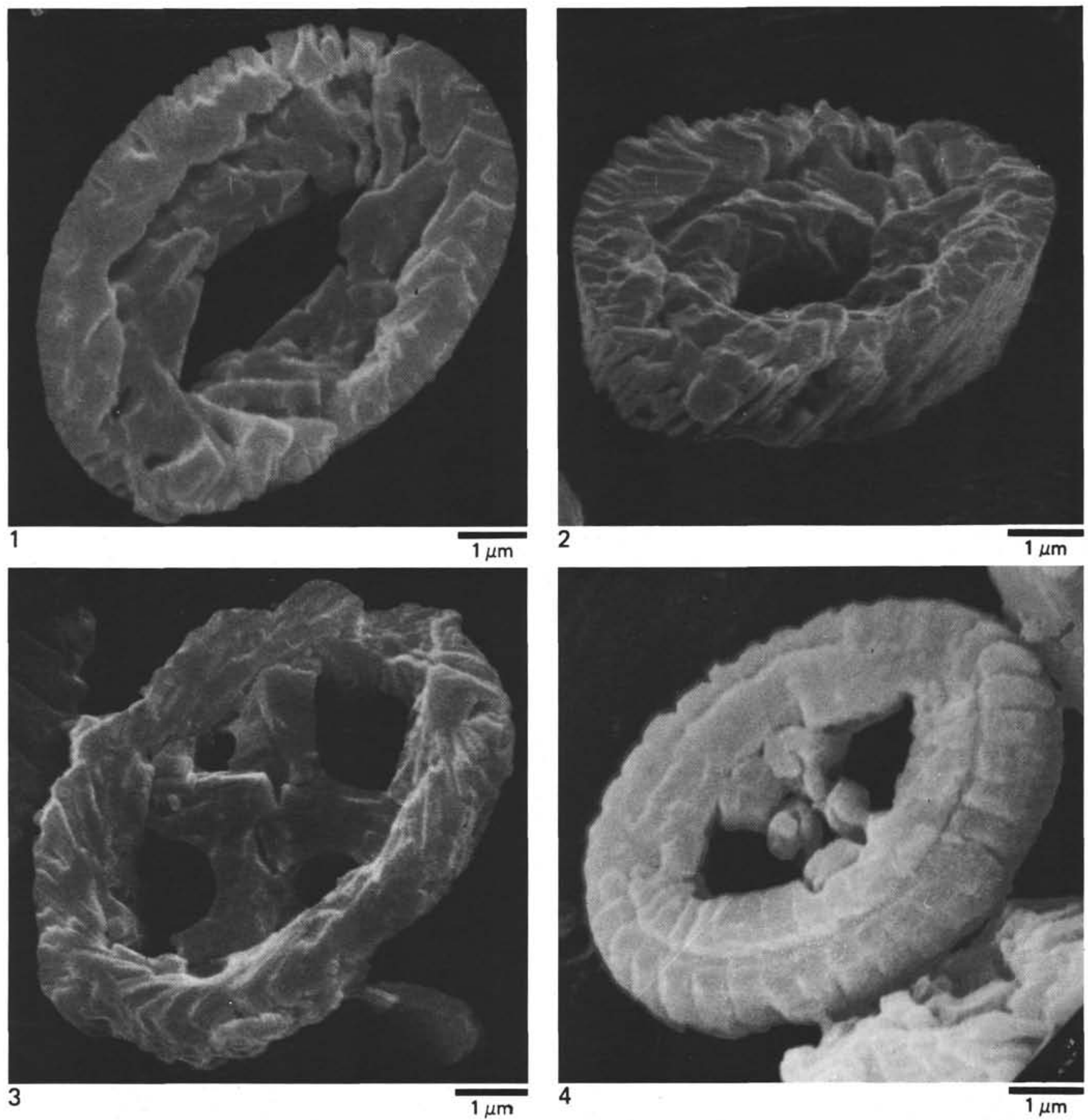

Plate 49. 1. Zygodiscus sp., distal view, etched specimen, Sample 530A-43-2, 20-21 cm, SEM; 2. Zygodiscus sp., side view, etched specimen, Sample 530A-43-2, 20-21 cm, SEM; 3. Neococcolithites protenus, distal view, Sample 530A-43-2, 20-21 cm, SEM; 4. Zygodiscus cf. Z. spiralis, proximal view, Sample 530A-50-2, 13-14 cm, ? reworked, SEM. 

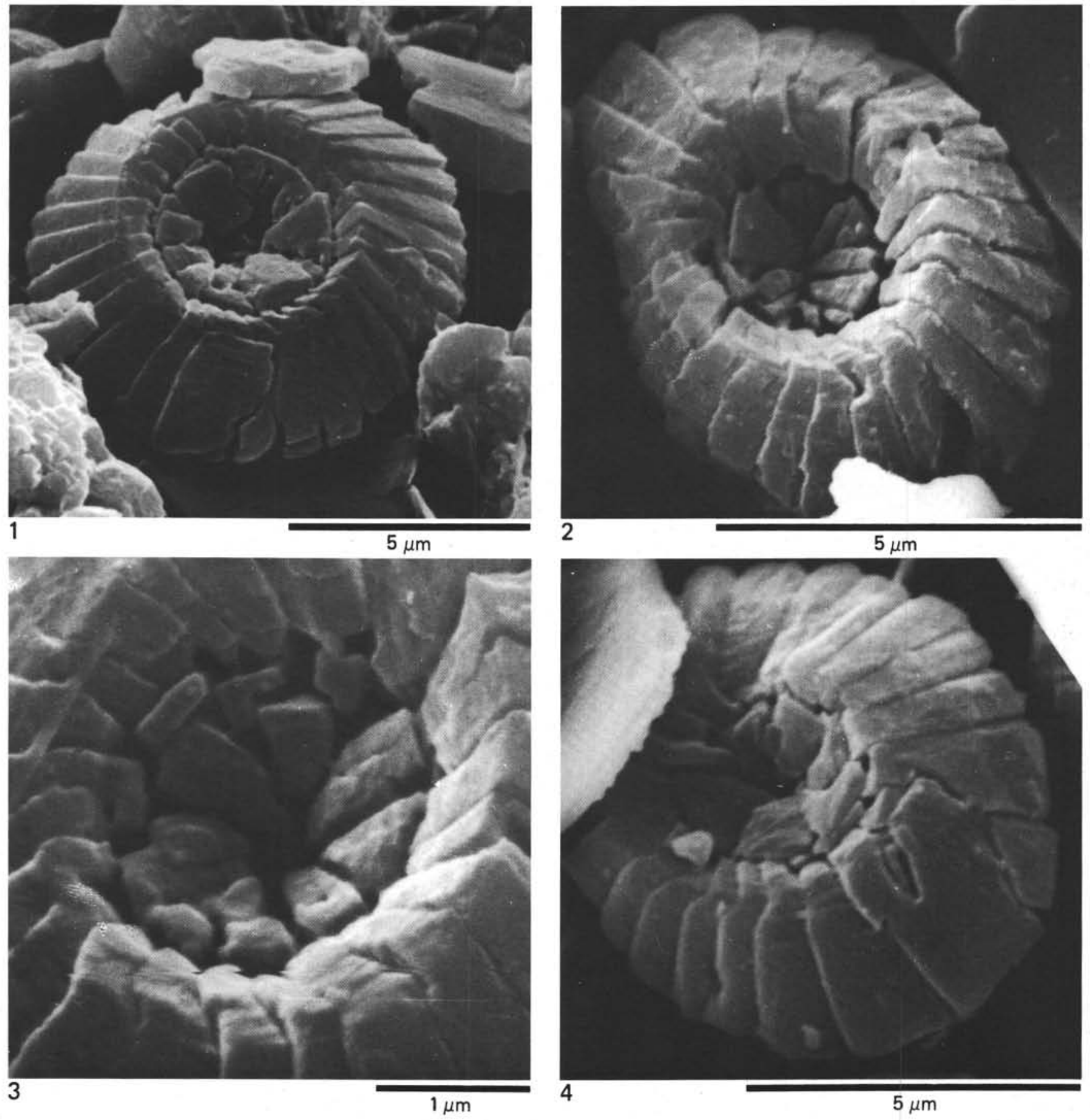

Plate 50. 1-4. Markalius astroporus, Sample 530A-50-1, 6-7 cm, SEM. (1) distal view; (2) distal view; (3) central detail, distal view; (4) distal view. 

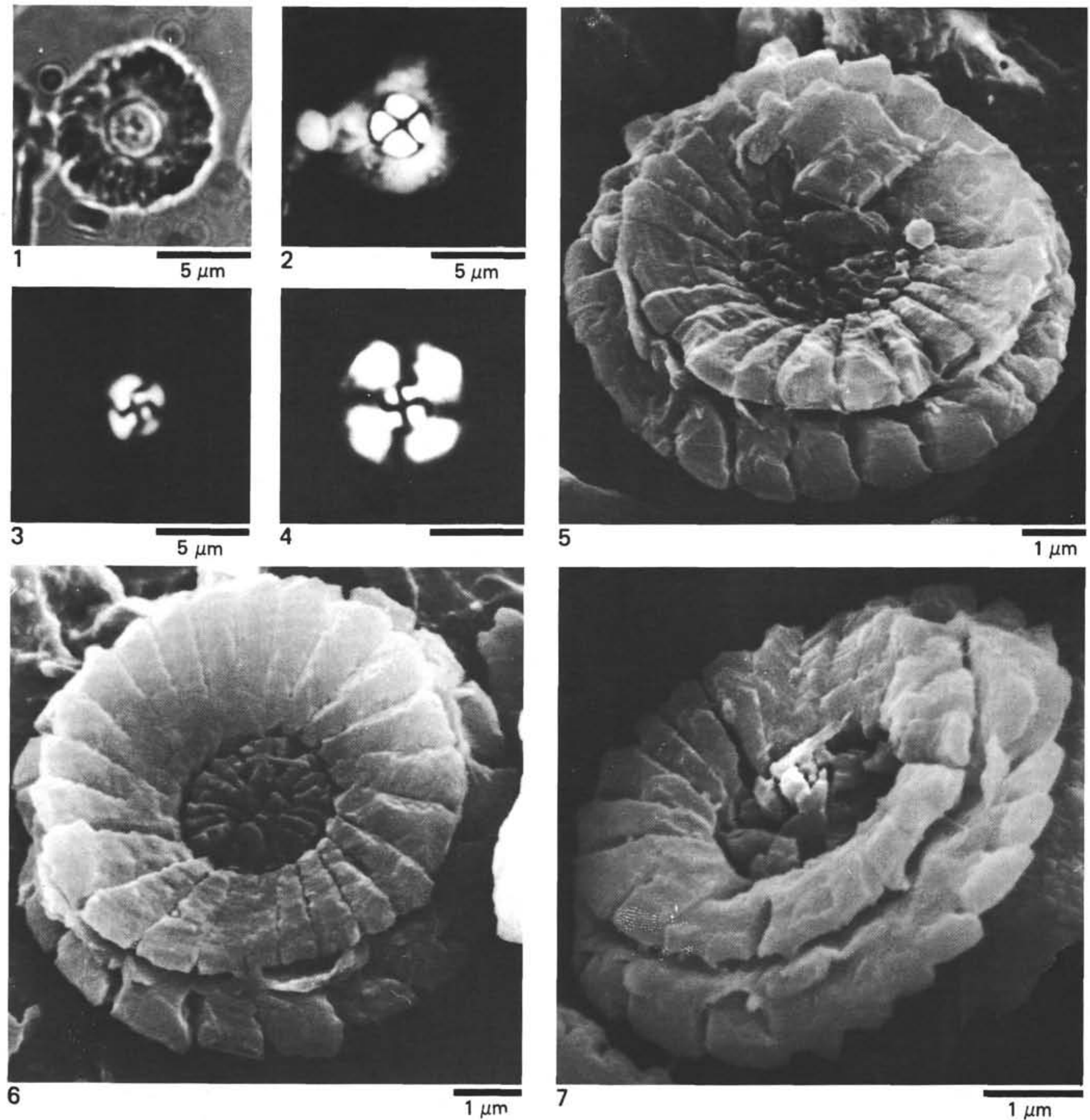

Plate 51. 1. Markalius astroporus, Sample 530A-50-1, 6-7 cm, NL; 2. M. astroporus, Sample 530A-47-1, 142-143 cm, XN; 3-4. Cyclagelosphaera reinhardtii, Sample 530A-50-1, 6-7 cm, XN; 5-6. Markalius astroporus, proximal view, Sample 530A-50-1, 6-7 cm, SEM; 7. M. astroporus, oblique proximal view, Sample 530A-50-1, 6-7 cm, SEM. 


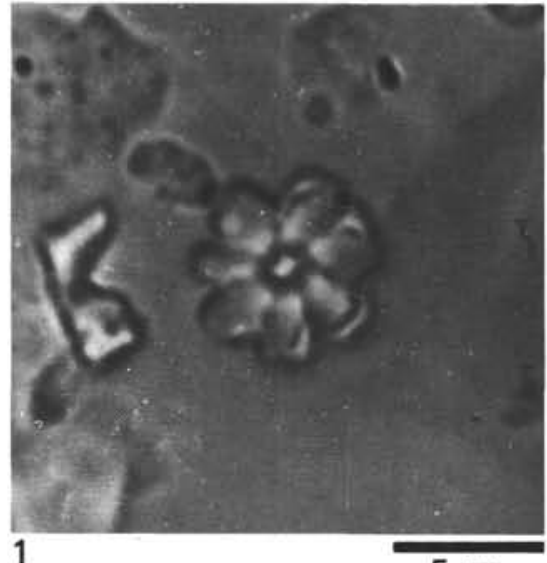

1

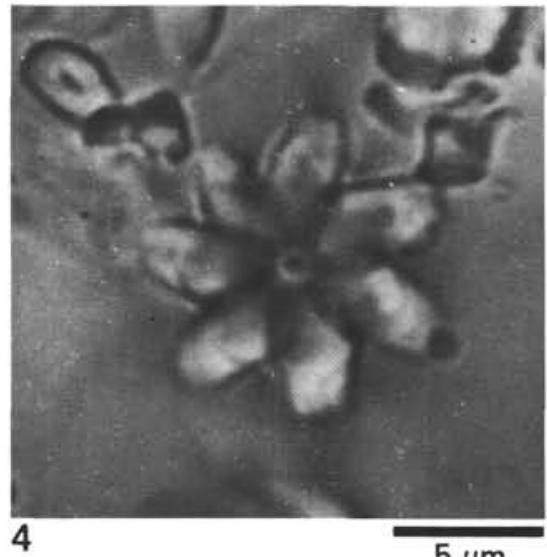

4

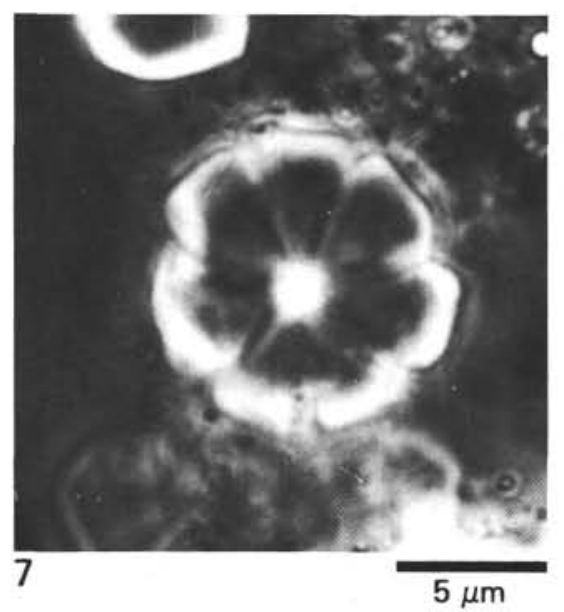

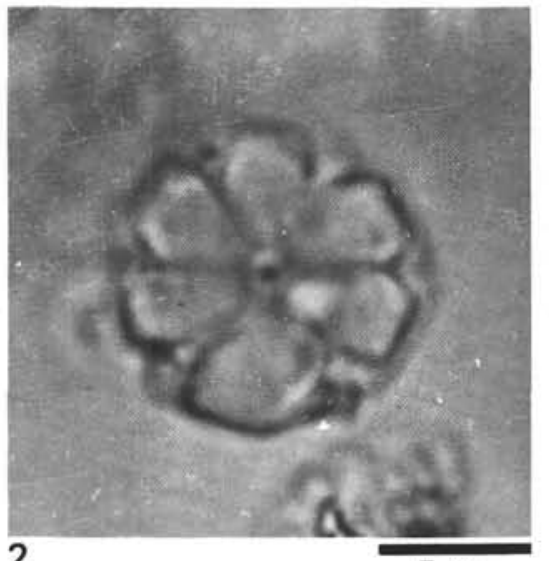

$5 \mu \mathrm{m}$
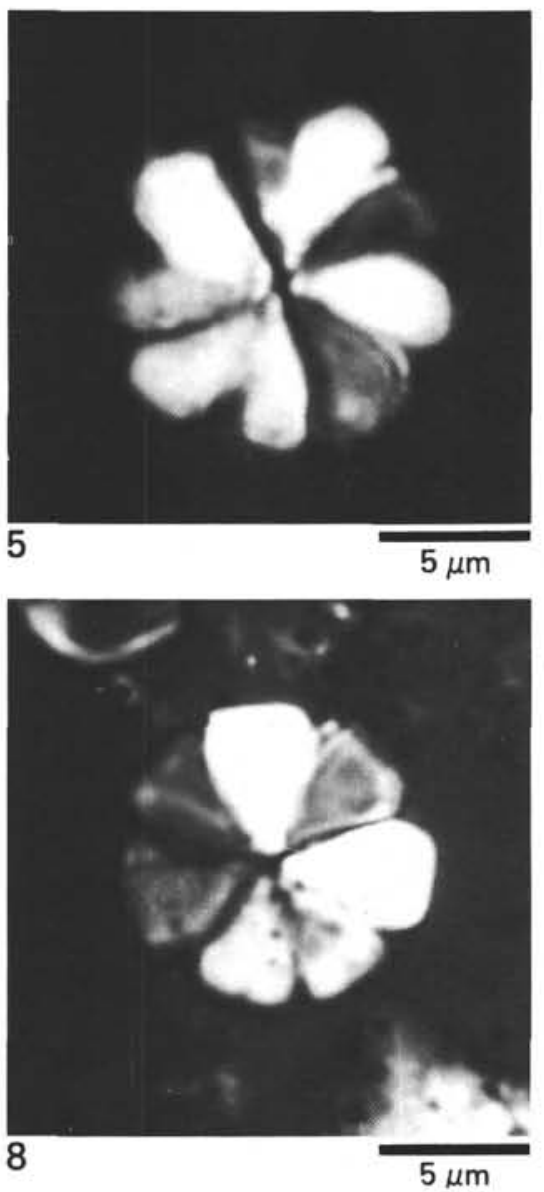
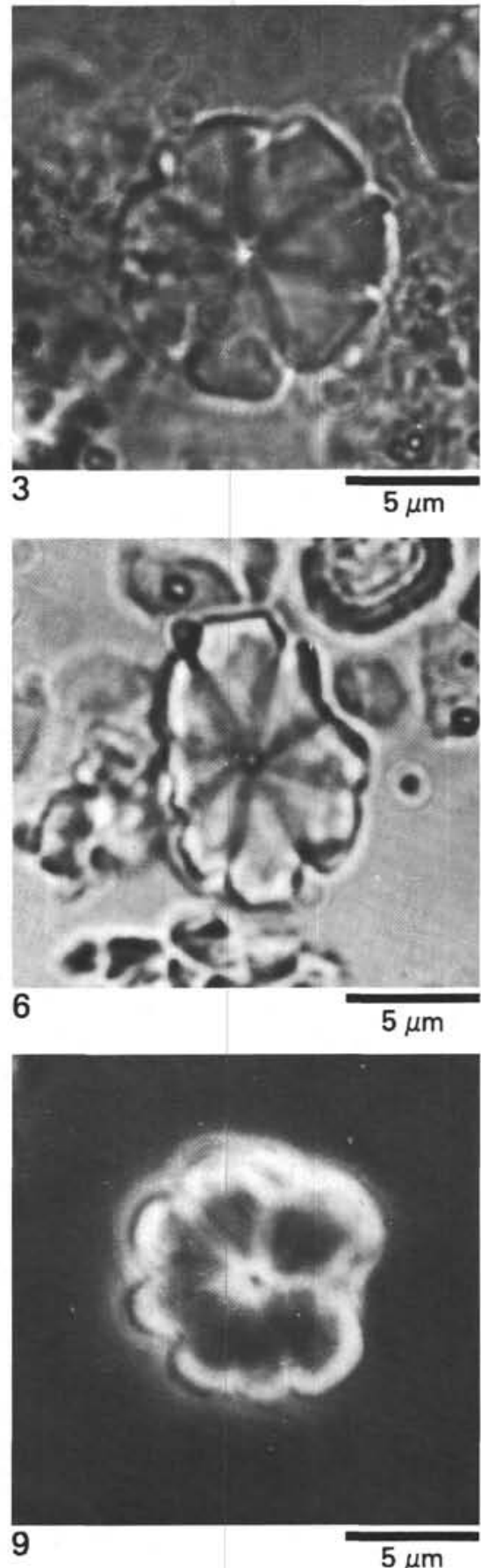

Plate 52. 1. Biantholithus astralis nov. spec., Sample 530A-50-2, 13-14 cm, NL; 2-3. B. sparus, Sample 530A-50-2, 13-14 cm, NL; 4. B. astralis nov. spec., Sample 530A-50-2, 13-14 cm, NL; 5-9. B. sparsus, (5) Sample 530A-50-1, 6-7 cm, XN, (6) Sample 530A-50-1, 6-7 cm, NL, (7) Sample 530A-50-2, 13-14 cm, PHC, (8) Sample 530A-50-2, 13-14 cm, XN, (9) Sample 530A-50-2, 13-14 cm, PHC. 

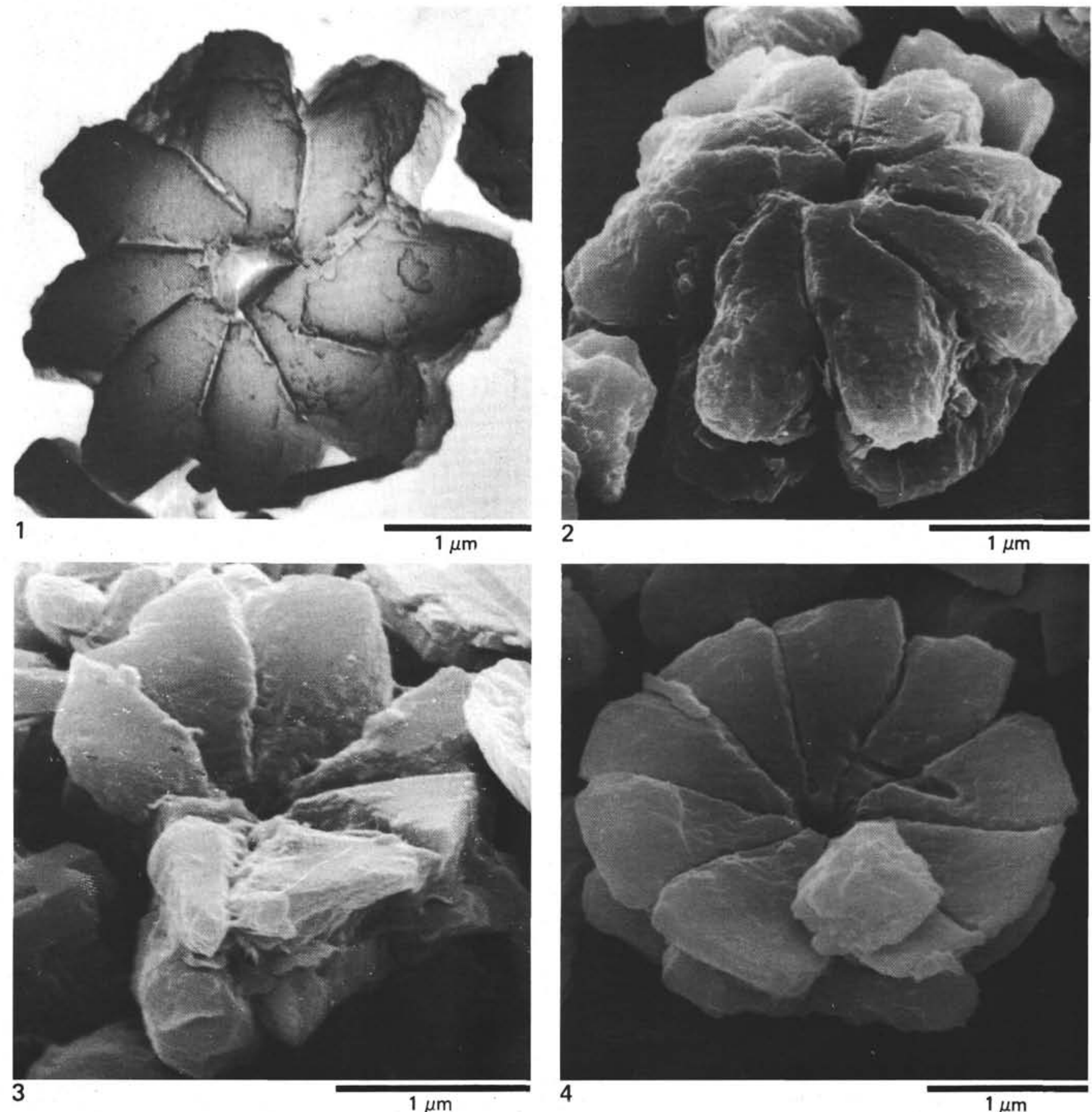

Plate 53. Sample 530A-50-1, 6-7 cm. 1. Biantholithus astralis nov. spec., holotype, SEM; 2. B. astralis nov. spec., SEM; 3-4. B. sparus, SEM. 

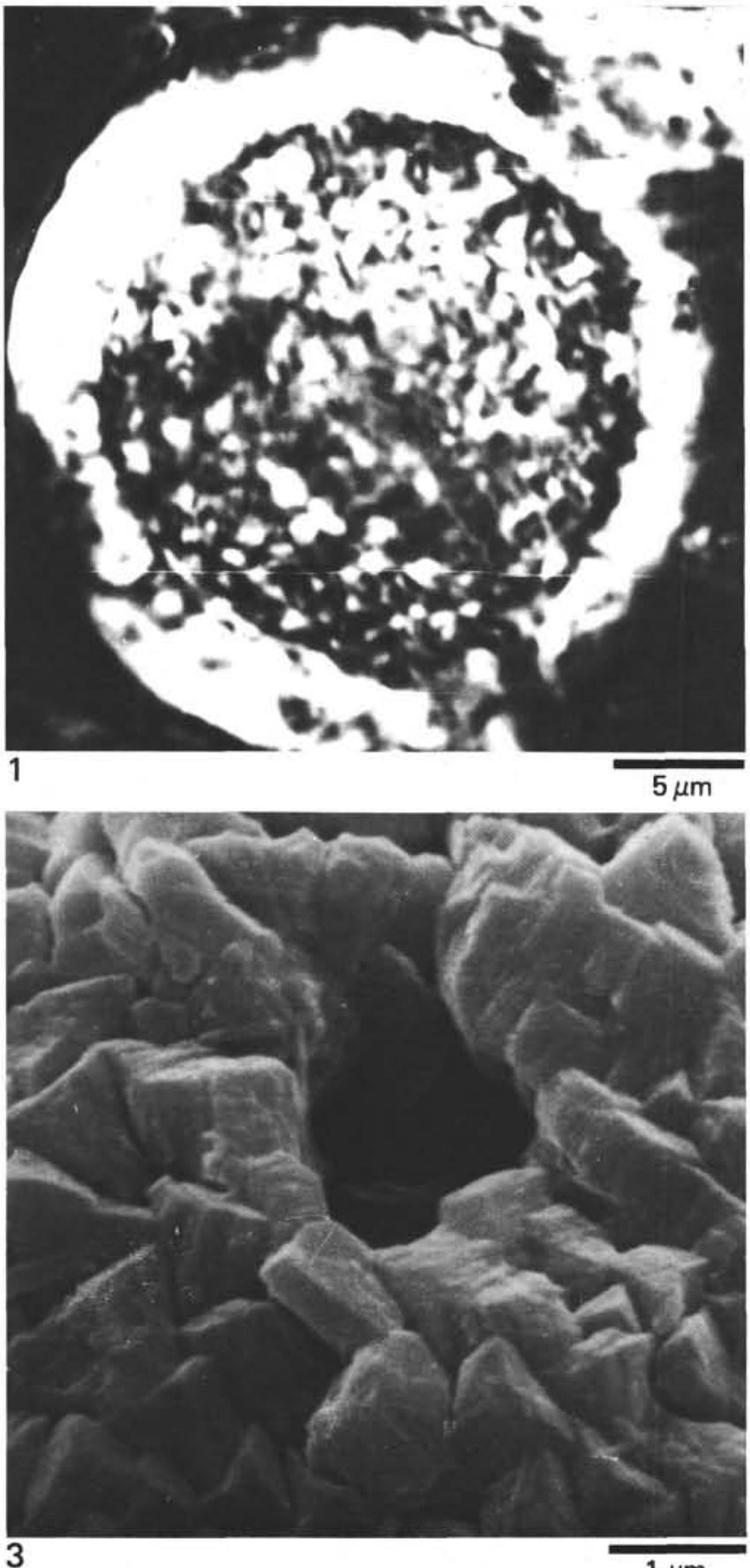

$1 \mu \mathrm{m}$
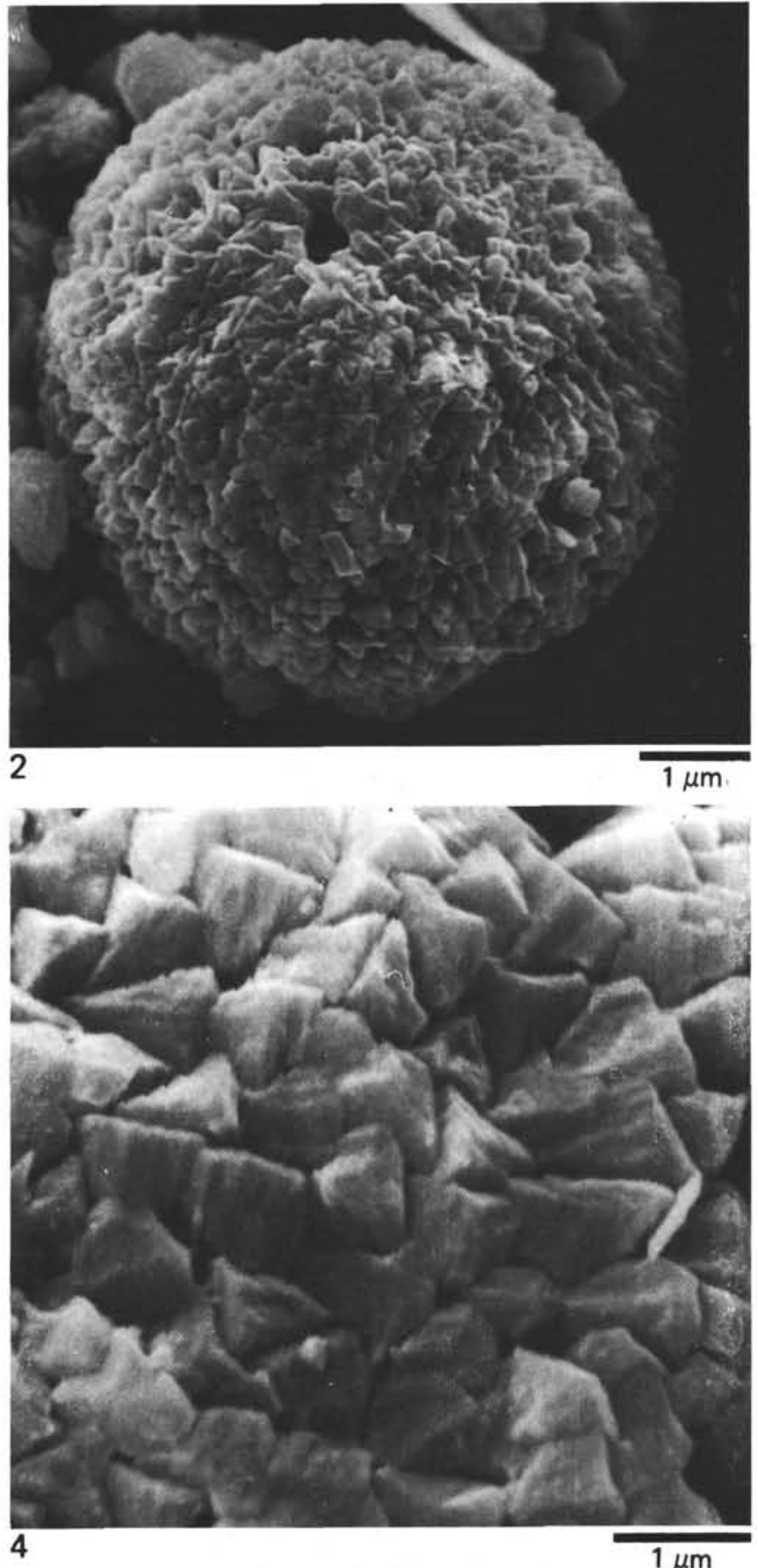

$1 \mu \mathrm{m}$

Plate 54. Thoracosphaera cf. T. operculata. 1. Complete cyst, Sample 530A-50-2, 13-14 cm, XN; 2. Complete cyst, Sample 530A-50-1, 6-7 cm, SEM; 3. Closeup detail of outer wall surface of specimen shown in Figure 2; 4. Closeup detail of outer wall surface of specimen shown in Figure 2. 